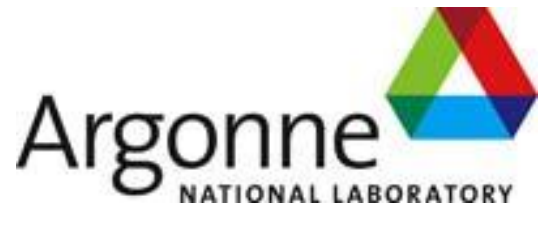

\title{
ANL-11/TRACC-USDOT-Y5Q4
}

\section{Transportation Research and Analysis Computing Center (TRACC) Year 5 Quarter 4 Progress Report}

Energy Systems Division 


\begin{abstract}
About Argonne National Laboratory Argonne is a U.S. Department of Energy laboratory managed by UChicago Argonne, LLC under contract DE-AC02-06CH11357. The Laboratory's main facility is outside Chicago, at 9700 South Cass Avenue, Argonne, Illinois 60439. For information about Argonne and its pioneering science and technology programs, see www.anl.gov.
\end{abstract}

\title{
Disclaimer
}

This report was prepared as an account of work sponsored by an agency of the United States Government. Neither the United States Government nor any agency thereof, nor UChicago Argonne, LLC, nor any of their employees or officers, makes any warranty, express or implied, or assumes any legal liability or responsibility for the accuracy, completeness, or usefulness of any information, apparatus, product, or process disclosed, or represents that its use would not infringe privately owned rights. Reference herein to any specific commercial product, process, or service by trade name, trademark, manufacturer, or otherwise, does not necessarily constitute or imply its endorsement, recommendation, or favoring by the United States Government or any agency thereof. The views and opinions of document authors expressed herein do not necessarily state or reflect those of the United States Government or any agency thereof, Argonne National Laboratory, or UChicago Argonne, LLC. 


\section{Transportation Research and Analysis Computing Center (TRACC) Year 5 Quarter 4 Progress Report}

by

H. Ley

Energy Systems Division, Argonne National Laboratory

submitted to

D. Tucker-Thomas

Office of Research Development \& Technology

Research and Innovative Technology Administration

U.S. Department of Transportation

December 2011 
This page has been left blank intentionally 


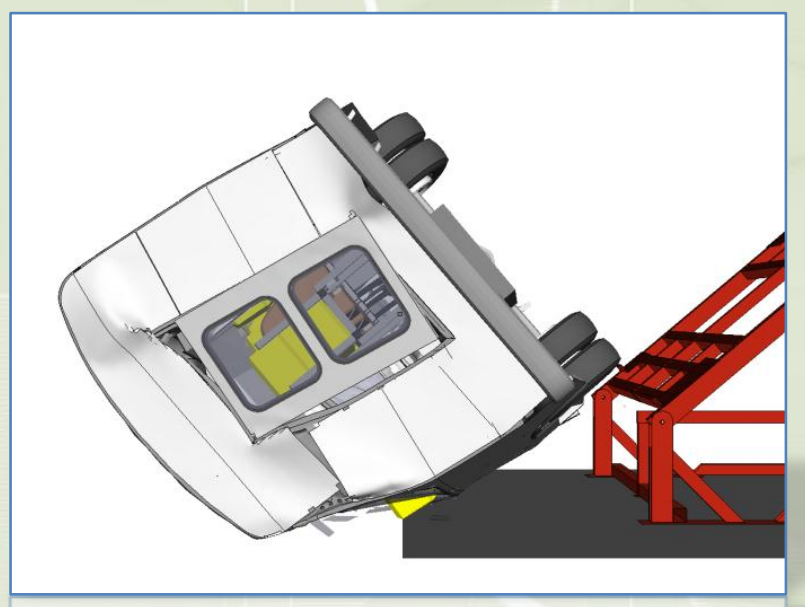

\section{Argonne Transportation}

Research and Analysis

Computing Center
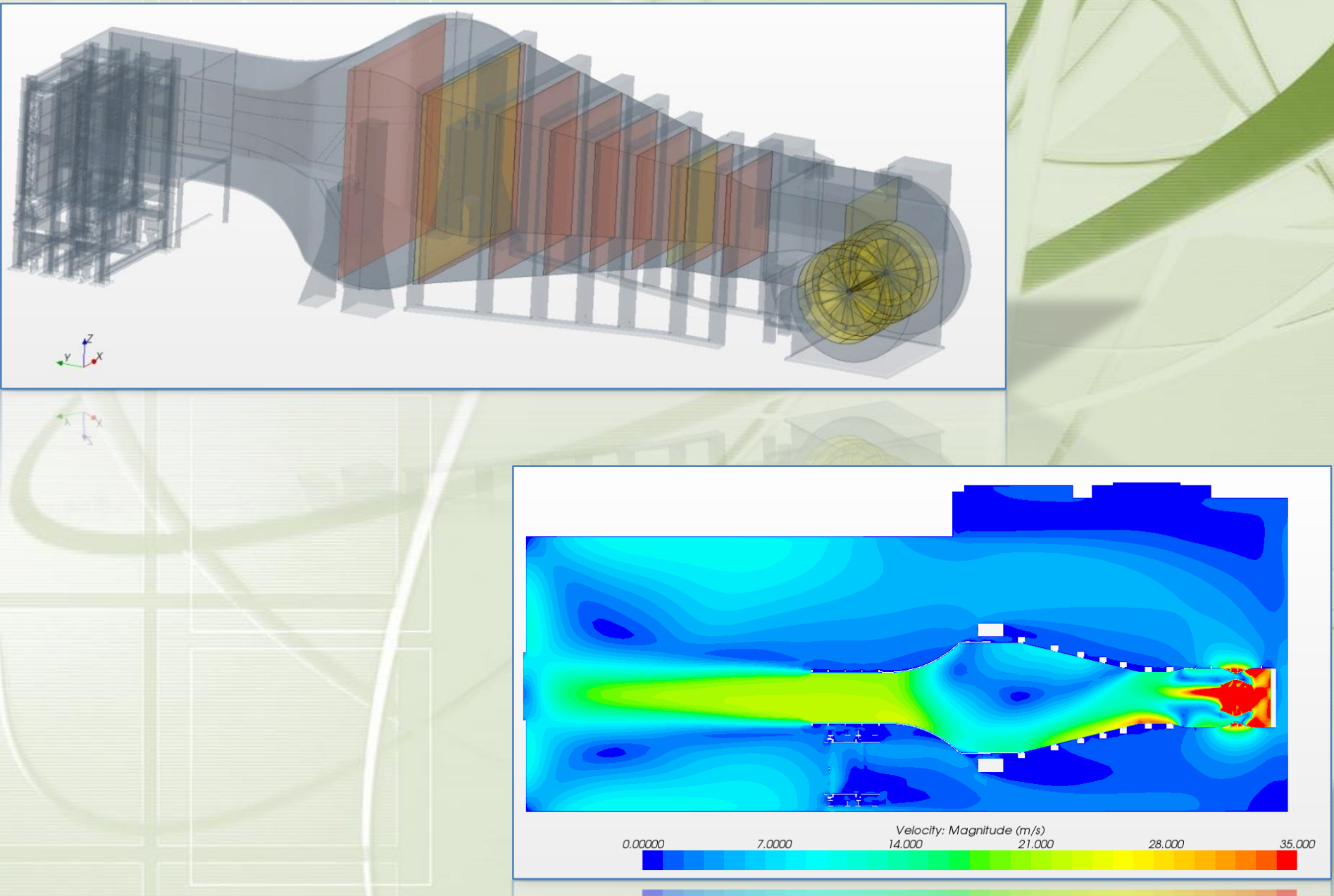

Quarterly Report

July through September 2011 



\section{Table of Contents}

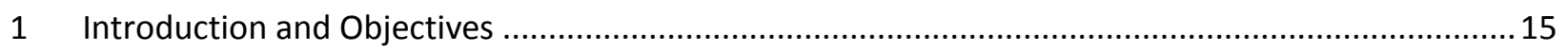

1.1 Operations, Computing, Networking, and Outreach Infrastructure, Facilities, and Capabilities .. 16

1.2 Transportation System Modeling and Evacuation Planning ..................................................... 16

1.3 Computational Fluid Dynamics for Hydraulic and Aerodynamic Research ............................... 17

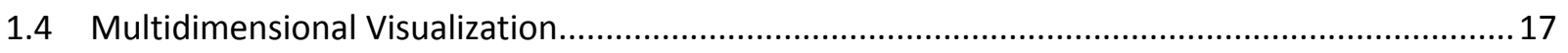

1.5 Computational Structural Mechanics Applications ............................................................. 18

2 Operations, Computing, Networking and Outreach Infrastructure, Facilities, and Capabilities ......... 19

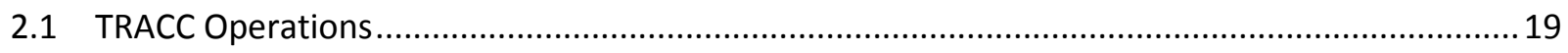

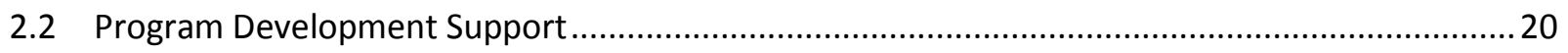

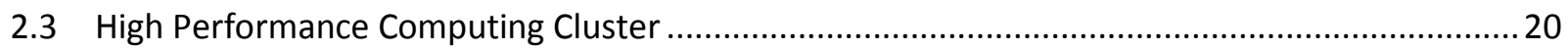

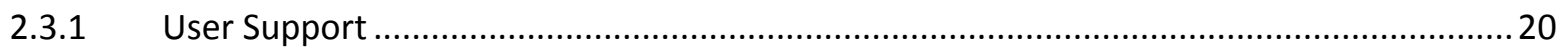

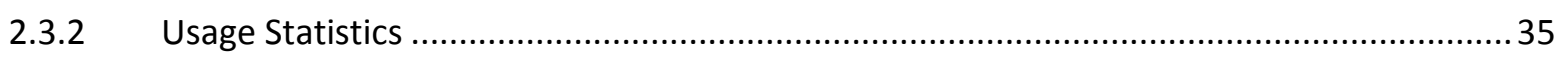

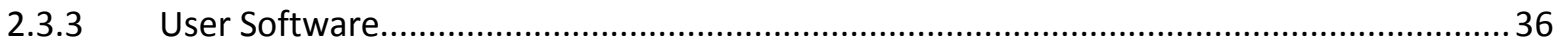

3 Transportation Systems Modeling and Evacuation Planning ..................................................... 38

4 Computational Fluid Dynamics for Hydraulic and Aerodynamic Research ....................................... 40

4.1 Technical Support of TRACC External Users in CFD Applications and Software ..........................40

1.1. Technical Contributions to the FHWA Bridge Hydraulics Analysis Program................................. 41

1.2. Three Dimensional Iterative Scouring Methodology Using Commercial CFD Software ................ 41

1.2.1. Computational Domain, Volume Mesh and Boundary Conditions................................... 41

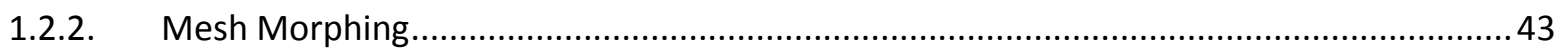

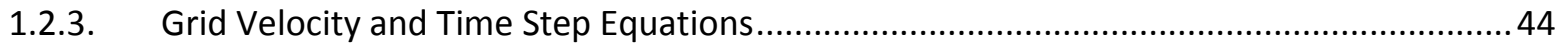

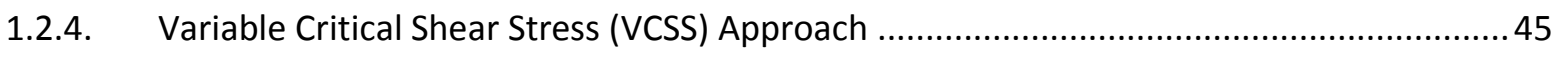

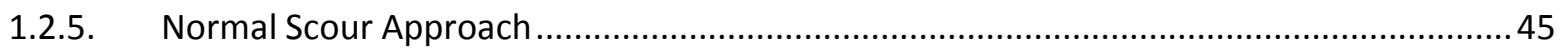

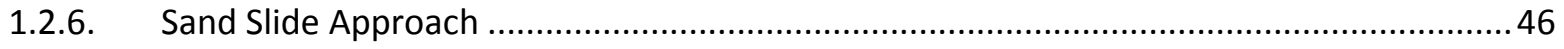

1.2.7. Field Functions for Scour Physics Models ..................................................................... 46

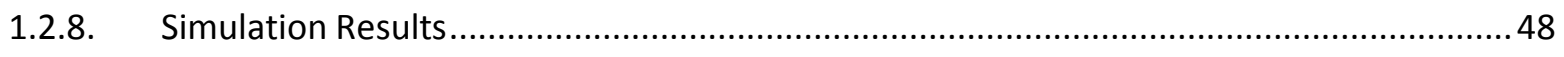

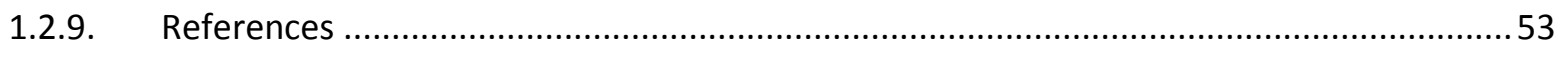

1.3. Outreach, User Support, Training and Technology Enablement ….........................................5 54 


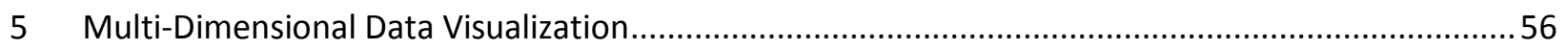

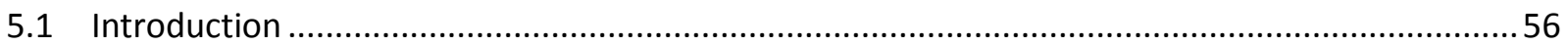

6 Computational Structural Mechanics Applications ….........................................................................5

6.1 Technical Support for Computational Structural Mechanics.......................................................58

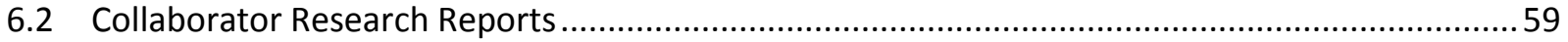

6.2.1 Florida Agriculture and Mechanical University - Florida State University College of

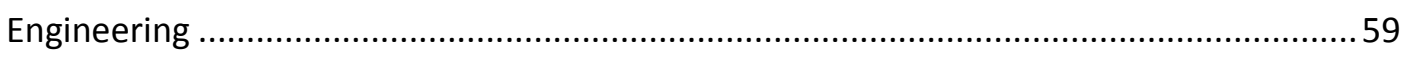

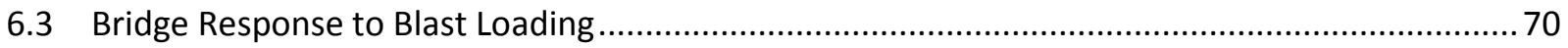

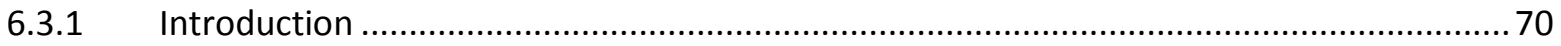

6.3.2 Simulation of Blast loading on a Square Reinforced Concrete Plate ................................ 70

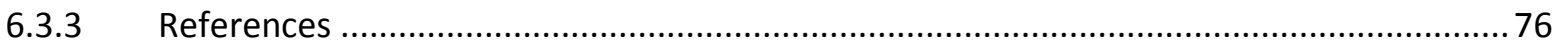

6.4 CSM Outreach, User Support, Training and Technology10.1080/13588265.2011.616118

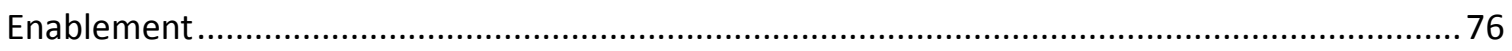

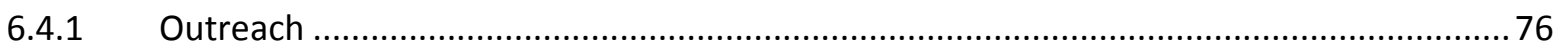

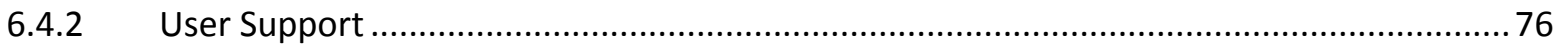

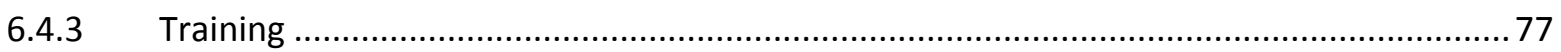

7 Work for Others - RTSTEP - Regional Transportation Simulation Tool for Evacuation Planning ......... 79

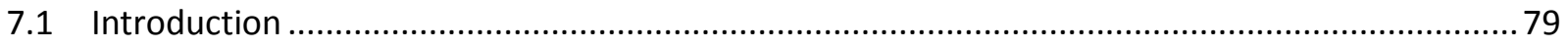

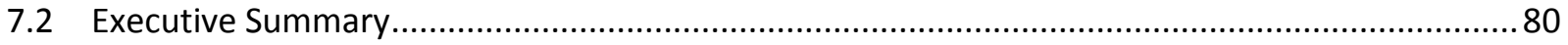

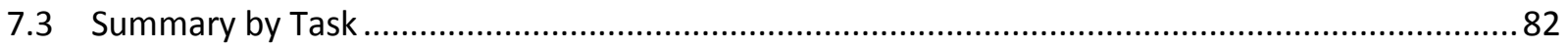

7.3.1 Task 1: Refinement and Extension of the Existing Road and Transit Network ................. 82

7.3.2 Task 2: Development of Tools to Evaluate Evacuation Routes and Strategies ..................85

7.3.3 Task 3: Tools to Model Populations, Evacuation Trips and Background Traffic ...............87

7.3.4 Task 4: Development of Visualization Methodologies to Compare Scenarios .................. 89

7.3.5 Task 5: Integration of the Transportation Model with the Dispersion Model...................92

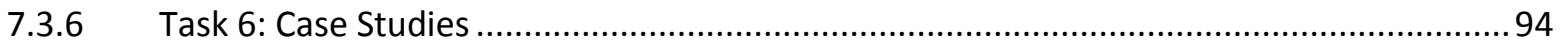

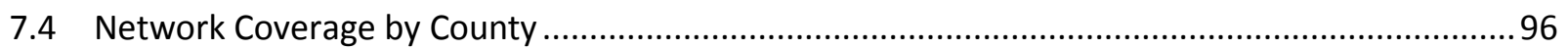

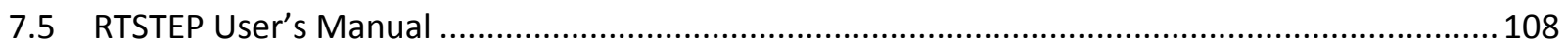

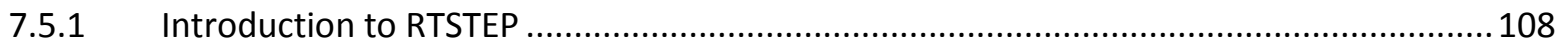

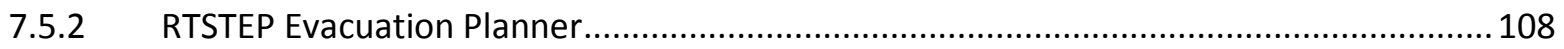

7.5.3 RTSTEP Evacuation Configuration Tool ................................................................... 115

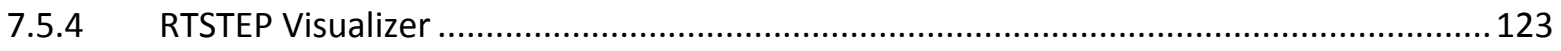


8 Work for Others - Computational Mechanics Research and Support for Aerodynamics and

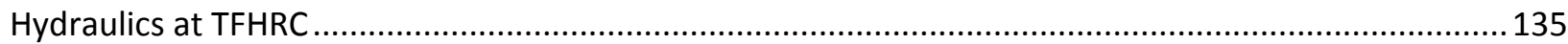

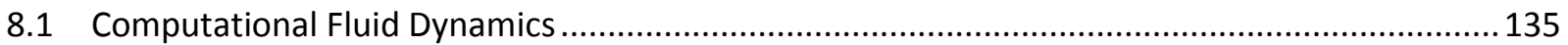

1.3.1. CFD Analysis of Flow Separation under an Inundated Bridge Deck for Application to

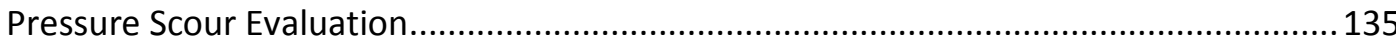

1.3.2. Computational Modeling and Analysis of Flow through Large Culverts for Fish Passage 144

1.3.3. Initial Modeling of the Wind Tunnel Laboratory at TFHRC ............................................ 162

1.3.4. Modeling of Truck Generated Salt Spray under Bridge with Sliding Mesh......................173

8.2 Computational Multiphysics Mechanics....................................................................................... 189

8.2.1 Multiphysics Simulation of Salt Spray Transport ........................................................ 189

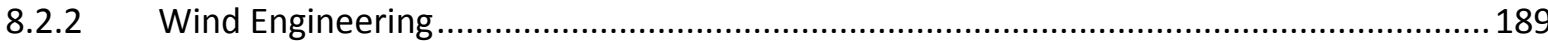

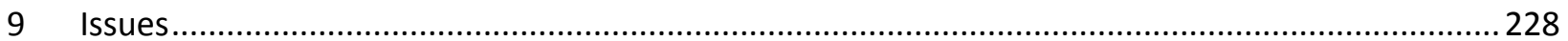

10 Financial Summary - Transportation Research and Analysis Computing Center for USDOT ...........230 


\section{List of Figures}

Figure 2.1: Daily Cluster Node Usage with Min and Max Number of Nodes since 2008.......................... 35

Figure 2.2: Relative Daily Cluster Node Usage by Application Area ..................................................... 36

Figure 4.1: Computational domain with key dimensions labeled .................................................... 42

Figure 4.2: Volume mesh of computational domain used in simulations ............................................ 43

Figure 4.3: Schematic of normal scour approach showing representative sediment bed cell face .......... 46

Figure 4.4: Initial bed geometry showing positions used to obtain data for plotted results. .................. 49

Figure 4.5: Solver time per time step as a function of the number of cluster compute nodes using 8

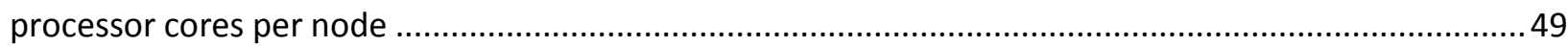

Figure 4.6: Sediment bed contours after 2 hours of scour with all 3 model enhancements showing bowl

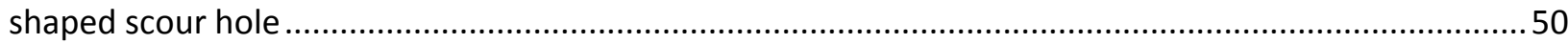

Figure 4.7: Evolution of scour hole profiles across the bed from ( $x=0$ line) from the edge of the pier at $z$

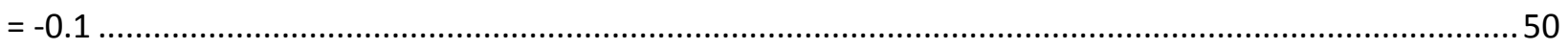

Figure 4.8: Sediment bed shear stress magnitude after 2 hours of scour...........................................51

Figure 4.9: Sediment bed critical shear stress after 2 hours of scour showing low values on downslopes and higher values on up slopes with respect to horizontal in the direction of the shear stress vector .... 51

Figure 4.10: Bed slope angle after 2 hours of scour ........................................................................ 51

Figure 4.11: Comparison of scour hole contours after 2 hours of scour with successive model enhancements: (a) uses only variable critical shear stress, (b) adds scour displacement normal to the

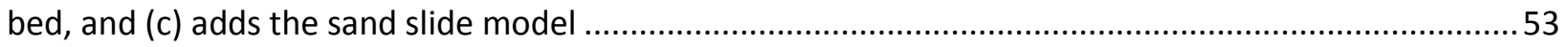

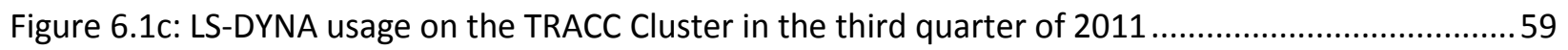

Figure 6.2: Comparison of FE simulations with and without adjustment of COF of concrete slab ............60

Figure 6.3: Coefficient of friction (COF) of wet concrete slab calibration results. Time vs. displacement in the $y$ direction of the selected node, multiple iterations have been shown...........................................61

Figure 6.4: Optimization history for coefficient of friction (fparam) of wet concrete slab......................61

Figure 6.5: Comparison of PVB formulation for different parameters ................................................. 63 
Figure 6.6: A comparison of results from the initial FE simulation of $800 \mathrm{~mm}$ rollover test (top) and after calibration (bottom)

Figure 6.7: Comparison of experimental and numerical results from the $800 \mathrm{~mm}$ rollover .64

Figure 6.8: Comparison of string transducer displacement and numerical results from an $800 \mathrm{~mm}$ rollover

Figure 6.9: Comparison of string transducer displacement and numerical results from a $909 \mathrm{~mm}$ rollover

Figure 6.10: Additional columns and plates connecting cab chassis and bus cage implemented into the model - original design on left, improved design on right 67

Figure 6.11: Design change of the steel doorframe - original design on left, improved design on right ...67

Figure 6.12: Final deformation of a bus structure - original design on left, improved design on right......69

Figure 6.13: Test specimen geometry and reinforcement details 71

Figure 6.14: Damage in the plate after the blast event for different LS-DYNA material models .74

Figure 6.15: Approximate location of the cracks in the experiments .75

Figure 7.1: Extent of the Chicago Metropolitan Area highway network used for RTSTEP 98

Figure 7.2: Cook County 99

Figure 7.3: Northern Cook County 100

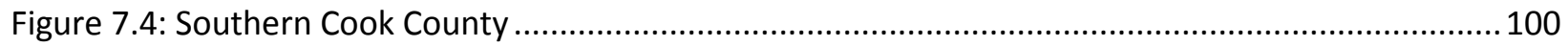

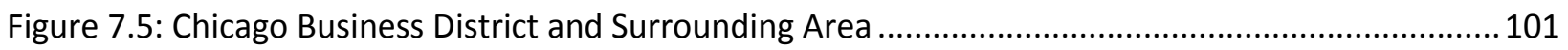

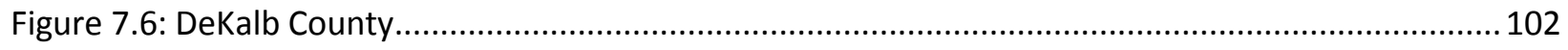

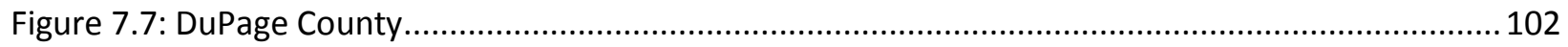

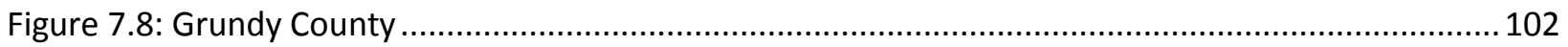

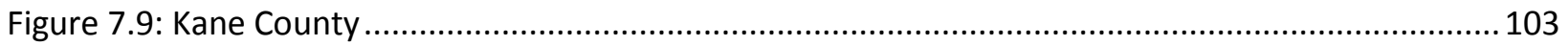

Figure 7.10: Kankakee County (based on CMAP and other regional data) ..........................................103

Figure 7.11: Kankakee County (detailed data layers have been made available) ................................. 103

Figure 7.12: Kankakee (detailed data layers have been made available) ............................................ 104

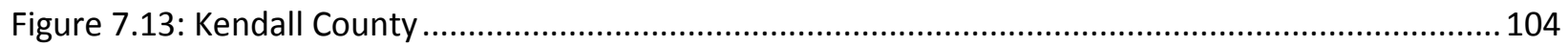




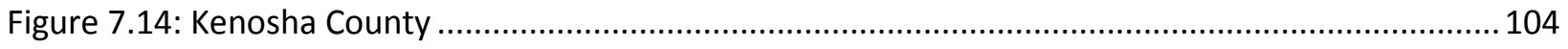

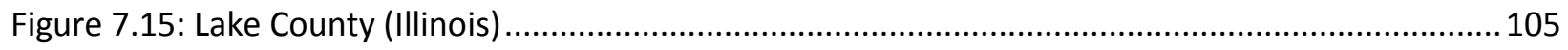

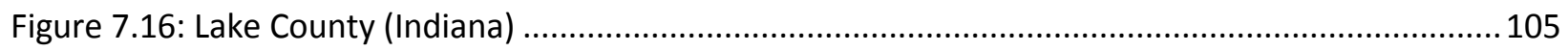

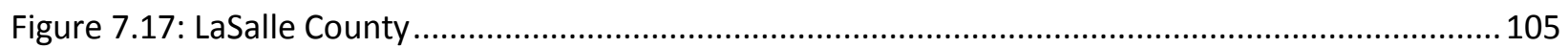

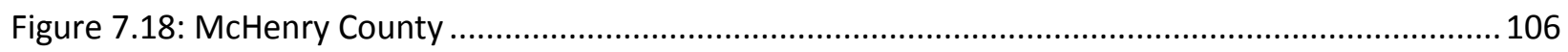

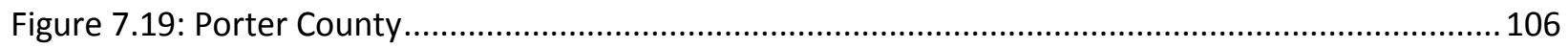

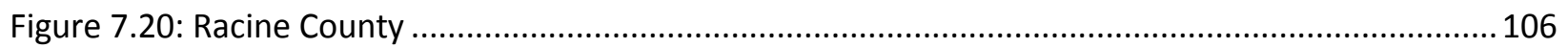

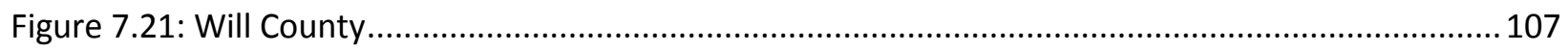

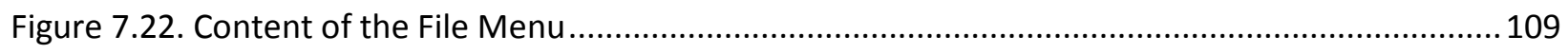

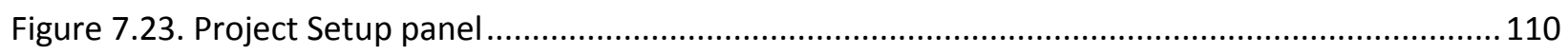

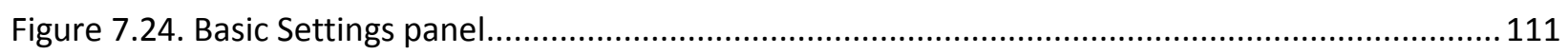

Figure 7.25. Transims Settings in the Advanced Settings menu ....................................................... 112

Figure 7.26. Scenario Settings in the Advanced Settings menu...................................................... 113

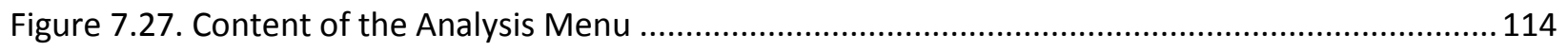

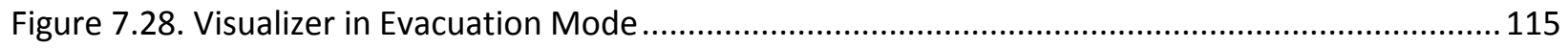

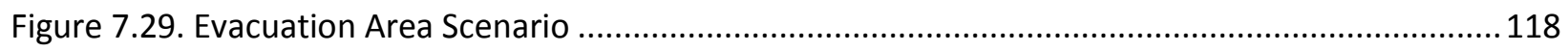

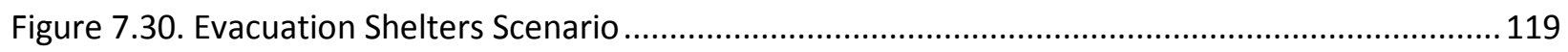

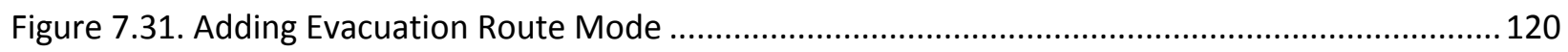

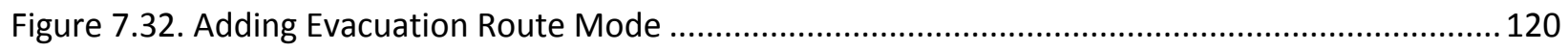

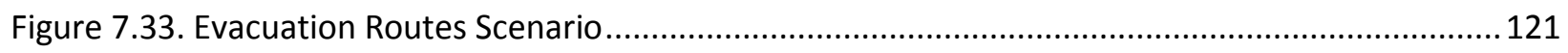

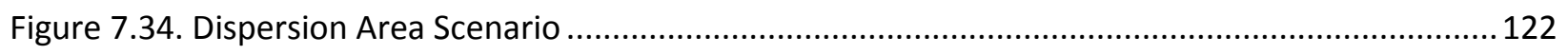

Figure 7.35. The GUI will have three frames: a Map Options Frame on the left, a frame showing the network on the right, and a TimeBar frame at the bottom. ........................................................... 124

Figure 7.36. An example of shape file displayed with the color gradation. ........................................ 132

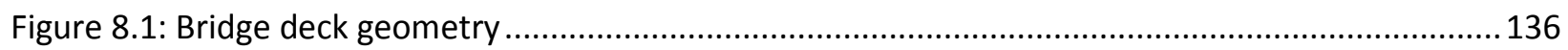

Figure 8.2: Schematic of flow domain for separation zone simulations ............................................136

$\begin{array}{ll}\text { TRACC/USDOT Y5Q4 } & \text { Page } 7\end{array}$ 
Figure 8.3: Symmetric section of bridge deck from center of a railing post to halfway to the next post 137

Figure 8.4: Velocity field under bridge deck before scour ............................................................... 138

Figure 8.5: Velocity field under bridge deck after scour ............................................................... 138

Figure 8.6: Velocity profiles upstream and $15 \mathrm{~cm}$ under the deck ...................................................139

Figure 8.7: Streamline bounding the separation zone under the deck before scour.............................140

Figure 8.8: Streamlines seeded from the upstream of the deck blockage showing the stagnation point before scour

Figure 8.9: Streamlines seeded from the upstream of the deck blockage showing the stagnation point with scour hole present

Figure 8.10: Separation zone bounding streamline for $h_{b}=0.05 \mathrm{~m}$ case 142

Figure 8.11: Separation zone bounding streamline for $h_{b}=0.30$ case

Figure 8.12: Vertical contraction of flow under bridge deck as a function of the height of the deck above the bed showing a dip at 0.47 when the deck is not fully submerged 143

Figure 8.13: Variation of flow velocity and depth in a cross-section of a corrugated metal pipe. 145

Figure 8.14: Depth-averaged velocity curve development in a cross-section of 6 inch water depth with

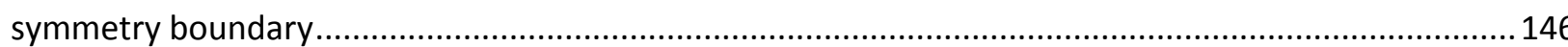

Figure 8.15: Flow path for the selected fish design criteria of velocity and depth ............................... 147

Figure 8.16: Cumulative area from the wall to the centerline......................................................... 148

Figure 8.17: Cumulative discharge from the wall to the centerline ................................................. 148

Figure 8.18: Cumulative discharge from the wall to the centerline in a cross-section of 6 inch water depth with symmetry boundary

Figure 8.19: Cumulative area from the wall to the centerline in a cross-section of 6 inch water depth with symmetry boundary..... 149

Figure 8.20: average velocity curve in a cross-section of 6 inch water depth with symmetry boundary 150 Figure 8.21 CAD model representing the computational domain used for porous media modeling ...... 152 Figure 8.22 Volumetric controls created around regions of major interest for mesh refinement .........154 Figure 8.23 Cross sectional view of the mesh scenes. 154 
Figure 8.24 Increased section of the culvert (nearly five times bigger than the reduced section) used for porous media modeling 155

Figure 8.25 Cross sectional view of mesh scene along on a plane taken along the length of the increased section 155

Figure 8.26 Image depicting the line probes created at a trough and a crest for a reduced section .......156

Figure 8.27 Image depicting the line probe created at a crest for an increased section ........................156

Figure 8.28 Velocity profiles for both reduced and increased sections at a crest..................................157

Figure 8.29 Velocity profiles for both reduced and increased sections at a trough...............................158

Figure 8.30 Cross sectional plane created at a crest in the porous region for a reduced section ............159

Figure 8.31 Representation of odd numbered Uniform strips of $1 \mathrm{~cm}$ width created along the fluid section. 159

Figure 8.32 Surface-averaged velocity variation along the uniform strips plotted using "Threholds" ... 160

Figure 8.33: Velocity distribution over cross section at a crest showing the variation above the porous media gravel bed. 161

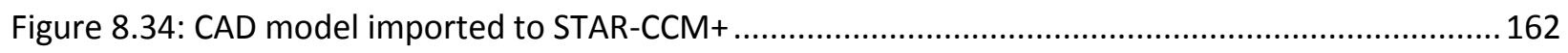

Figure 8.35: Geometry of the fan inlets: initial (left) and updated (right) ......................................... 163

Figure 8.36: Geometry of the fan: initial (left) and updated (right) ................................................. 163

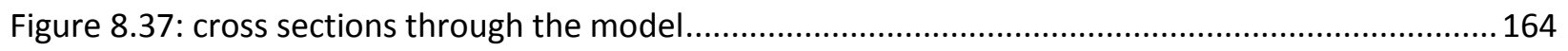

Figure 8.38: Velocity profile in model without the furniture - vertical plane...................................... 166

Figure 8.39: Velocity profile in model without the furniture - horizontal plane ................................... 166

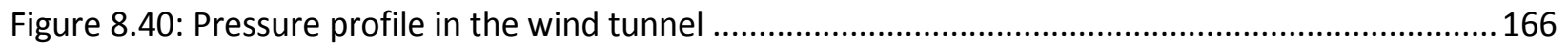

Figure 8.41: Velocity profile in model without the furniture and no screens - vertical plane................. 168

Figure 8.42: Velocity profile in model without the furniture and no screens - horizontal plane ............ 168

Figure 8.43: Velocity profile in model extended boundaries - horizontal plane ...................................169

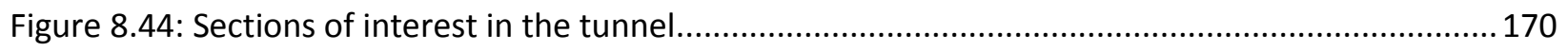

Figure 8.45: Area averaged standard deviation of the air velocity in the tunnel ................................. 171

Figure 8.46: Area averaged coefficient of variation of the air velocity in the tunnel............................ 171 
Figure 8.47: Geometry of the STAR-CCM+ model for analysis of truck generated salt spray

Figure 8.48: Schematic drawing of the Tire Spray Generator (based on [2]) ….................................. 175

Figure 8.49: Particle size distribution in Tire Spray Generator test [2] ............................................. 176

Figure 8.50: Possible particle behavior in Bai-Gosman model of wall impingement .............................177

Figure 8.51: Particle breakup in the wheel well ........................................................................... 178

Figure 8.52: Particle distribution after break-up in the wheel well................................................... 179

Figure 8.53: Location of surfaces used as surface injectors ......................................................... 180

Figure 8.54: Particle generated on the injector surfaces............................................................. 180

Figure 8.55: Velocity field around the truck and distribution of massless droplets injected at the cabin's

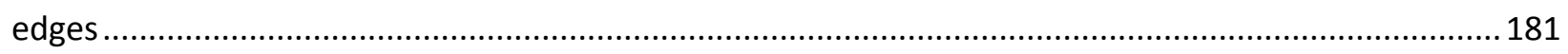

Figure 8.56: Velocity field around the truck and distribution of 50 micron droplets injected at the rear tire tread

Figure 8.57: Velocity field around the truck and distribution of 100 micron droplets injected at the rear tire tread 183

Figure 8.58: Velocity field around the truck and distribution of 50 micron droplets injected at the rear tire tread 184

Figure 8.59: Velocity field around the truck and distribution of 100 micron droplets injected at the rear tire tread..... 184

Figure 8.60: Velocity field around the truck and distribution of 100 micron droplets injected at all tire treads on one side of the vehicle. 185

Figure 8.61: Spray behind the truck driving on a wet road ...... 185

Figure 8.62: Velocity field around the truck and distribution of 100 micron droplets injected at all tire treads on one side of the vehicle. 185

Figure 8.63: Generation of the fine spray mist on the side of the truck 186

Figure 8.64: Velocity field around the truck and distribution of 100 micron droplets injected at all tire treads on one side of the vehicle..... 187

Figure 8.65: Count of droplets hitting the bridge beams during the simulation time 187

Figure 8.66: Geometry of the model with two trucks 188

Figure 8.67: Ballast portion [1] 190 
Figure 8.68: Wind influence face and rigid-wall attached with right wheel [1]

Figure 8.69: Configuration of SUT with driver side rear wheel liftoff.

Figure 8.70: Configuration of SUT in the process of rolling over...... 194

Figure 8.71: Critical driving speed for rollover on dry pavement $(\mu=0.9)$ 195

Figure 8.72: Critical driving speed for rollover on wet pavement $(\mu=0.5)$. 195

Figure 8.73: Critical driving speed for sideslip and rollover on dry pavement $(\mu=0.9)$ for a truck with a full cargo load. 196

Figure 8.74: Critical driving speed for sideslip and rollover on dry pavement $(\mu=0.9)$ for a truck with a $50 \%$ cargo load. 196

Figure 8.75: Critical driving speed for sideslip and rollover on dry pavement $(\mu=0.9)$ for a truck with $10 \%$ cargo load. 196

Figure 8.76: Critical driving speed for sideslip and rollover on wet pavement $(\mu=0.5)$ full cargo load.... 198

Figure 8.77: Critical driving speed for sideslip and rollover on wet pavement $(\mu=0.5)$ with $50 \%$ cargo load. 198

Figure 8.78: Critical driving speed for sideslip and rollover on wet pavement $(\mu=0.5)$ with $10 \%$ cargo load. 198

Figure 8.79: Critical driving speed for sideslip on icy pavement ( $\mu=0.1)$ with full cargo load) ................200

Figure 8.80: Critical driving speed for sideslip on icy pavement $(\mu=0.1)$ with $50 \%$ cargo load ................200

Figure 8.81: Critical driving speed for sideslip on icy payment $(\mu=0.1)$ with $10 \%$ cargo load ................. 200

Figure 8.82: Temporal contact force history for driver side rear tire under a $75 \mathrm{mph}$ crosswind ........... 201

Figure 8.83: Critical sustained time for initial liftoff on dry pavement $(\mu=0.9)$ with full cargo load ........ 202

Figure 8.84: Critical sustained time for rollover on dry pavement $(\mu=0.9)$ with full cargo load ..............202

Figure 8.85: Critical sustained time for initial liftoff on dry pavement $(\mu=0.9)$ with $50 \%$ cargo load ...... 203

Figure 8.86: Critical sustained time for rollover on dry pavement $(\mu=0.9)$ with $50 \%$ cargo load.............203

Figure 8.87: Critical sustained time for initial liftoff on dry pavement $(\mu=0.9)$ with $10 \%$ cargo load. ..... 204

Figure 8.88: Critical sustained time for rollover on dry pavement $(\mu=0.9)$ with $10 \%$ cargo load............. 204

Figure 8.89: Critical sustained time for initial liftoff on wet pavement $(\mu=0.5)$ with full cargo load. ...... 205 
Figure 8.90: Critical sustained time for rollover on wet pavement $(\mu=0.5)$ with full load. 205

Figure 8.91: Critical sustained time for initial liftoff on wet pavement $(\mu=0.5)$ with $50 \%$ cargo load. .... 206

Figure 8.92: Critical sustained time for rollover on wet pavement $(\mu=0.5)$ with $50 \%$ cargo load............ 206

Figure 8.93: Critical sustained time for initial liftoff on wet pavement ( $\mu=0.5)$ with $10 \%$ cargo load). ...207

Figure 8.94: Critical sustained time for rollover on wet pavement $(\mu=0.5)$ with $10 \%$ cargo load............ 207

Figure 8.95: Sensitivity analysis results for the initial model...........................................................208

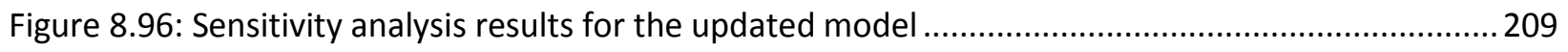

Figure 8.97: Approximate response surfaces for different values of pressure scaling factor .................2 210

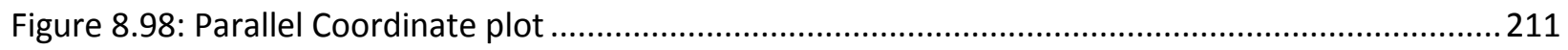

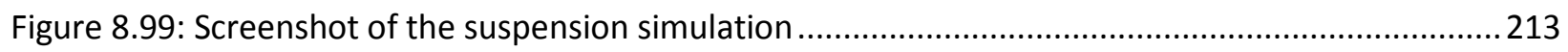

Figure 8.100: Force response vs. displacement of beam for front passenger location...........................213

Figure 8.101: Force response vs. displacement for the range of interest .......................................... 214

Figure 8.102: Force response vs. displacement of beam for rear driver location .................................215

Figure 8.103: Force response vs. displacement for the range of interest ........................................... 215

Figure 8.104: Front response vs. time for front driver location .........................................................216

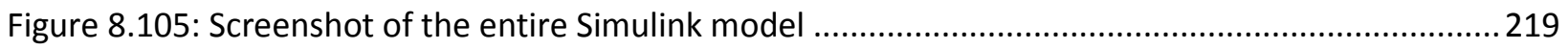

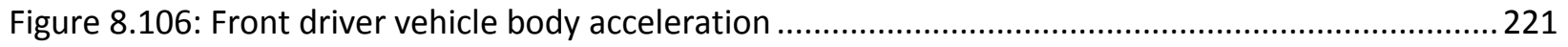

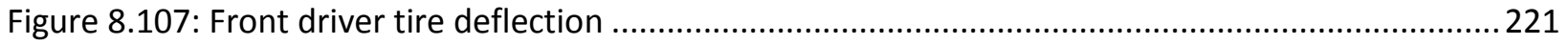

Figure 8.108: Front driver suspension deflection ....................................................................... 222

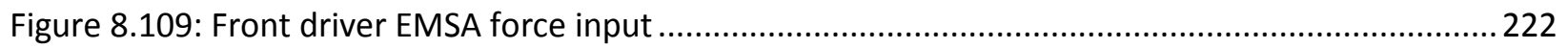

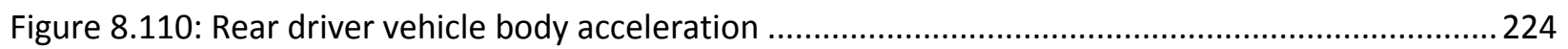

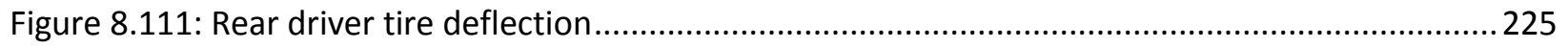

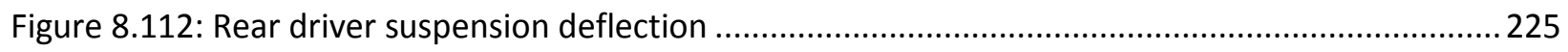

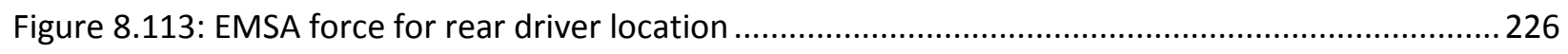




\section{List of Tables}

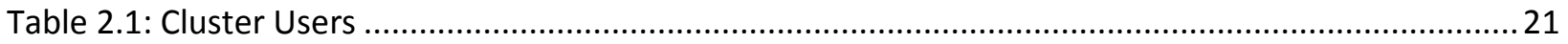

Table 2.2: Major Software Applications currently available on the TRACC cluster ................................. 37

Table 4.1 STAR-CCM+ field functions for scour physics models ....................................................... 47

Table 6.1: Deformation Index and total mass of a loaded vehicle for each design (iteration) ..................68

Table 6.2: Simplified LS-DYNA input for MAT_072R3 (units system metric ton, $\mathrm{mm}$, sec) ......................71

Table 6.3: Simplified LS-DYNA input for MAT_084 (units system metric ton, $\mathrm{mm}, \mathrm{sec}$ ) .......................... 72

Table 6.4: Simplified LS-DYNA input for MAT_159 (units system metric ton, $\mathrm{mm}$, sec) ......................... 72

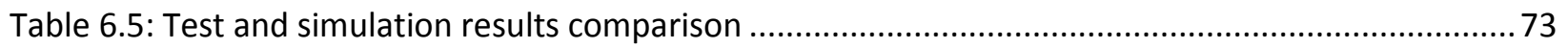

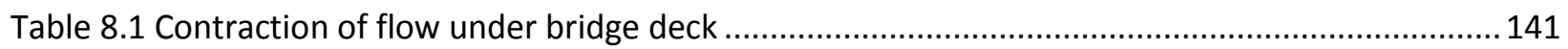

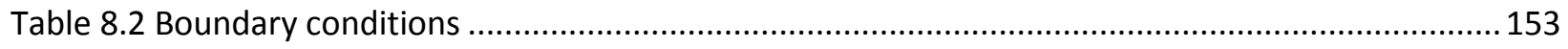

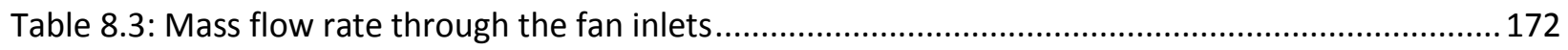

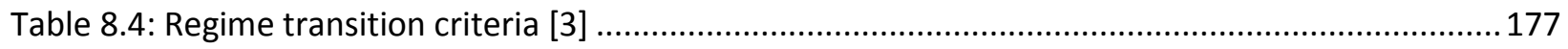

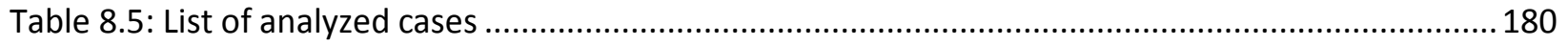

Table 8.6: Comparison of finite element model for a SUT to a Ford F800 truck...................................190

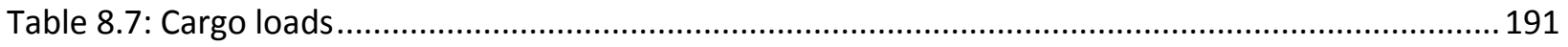

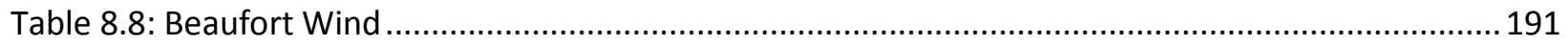

Table 8.9: Coefficient of friction for dry, wet and ice road conditions................................................ 192

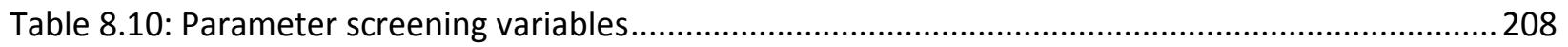

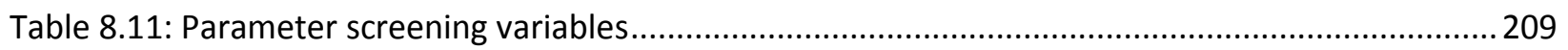

Table 8.12: Suspension stiffness values for each location ................................................................ 216

Table 8.13: Damping coefficients of the suspension for each location ...............................................216

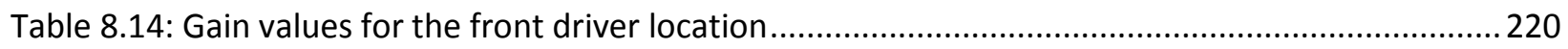

Table 8.15: RMS value comparison for the front driver location ......................................................220 


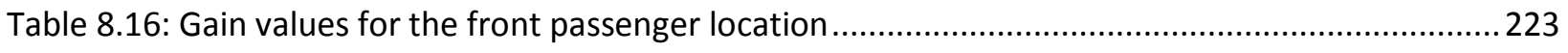

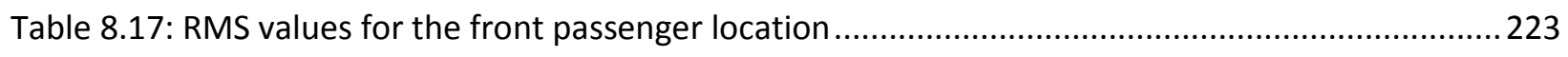

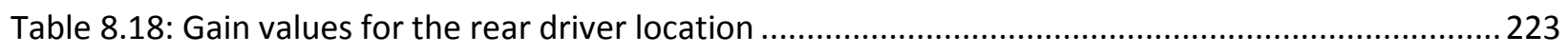

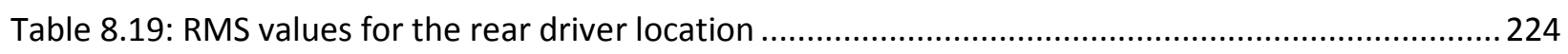

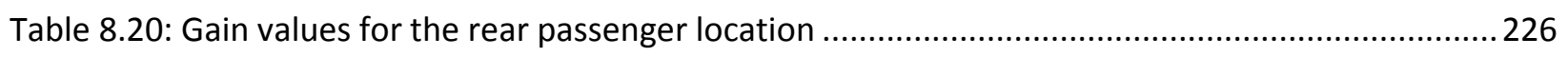

Table 8.21: RMS values for the rear passenger location .............................................................. 226 


\section{Introduction and Objectives}

Argonne National Laboratory initiated a FY2006-FY2009 multi-year program with the US Department of Transportation (USDOT) on October 1, 2006, to establish the Transportation Research and Analysis Computing Center (TRACC). As part of the TRACC project, a national high performance computer user facility has been established, with full operations initiated in March 2008. The technical objectives of the TRACC project include the establishment of a high performance computing center for use by USDOT research teams, including those from Argonne and their university partners, and the use of advanced computing and visualization facilities for the performance of focused computer research and development programs in areas of interest for USDOT. The project has been reauthorized for FY2010 and is now in its $5^{\text {th }}$ year. The project will not receive additional funding through the SAFETEA-LU grant any longer, but has been authorized to expend remaining FY2010 funds at Argonne to keep the TRACC facility operating at least until September 30, 2012.

The objectives have been met by establishing a high-performance computing facility, known as the Transportation Research and Analysis Computing Center (TRACC), and providing technical support for its use by USDOT staff and their university and industry contractors. In addition to facilities for advanced computing, visualization, and high-speed networking in the TRACC facility, advanced modeling and simulation applications research is being conducted by the TRACC facility scientific applications staff in coordination and collaboration with USDOT researchers.

This fourth quarter project report for Year 5 of the project (Y5Q4) summarizes progress on the principal activities associated with the operation of the computing center and in the performance of the computational research in the four key application areas identified by USDOT as its highest priorities. As defined by the Year 5 Statement of Work (SOW) the activities and objectives for the fifth year of the project are: (1) traffic modeling and simulation and emergency transportation planning; (2) computational fluid dynamics for hydraulics and aerodynamics research; (3) multi-dimensional data visualization; and (4) computational structural mechanics applications.

The establishment of the high performance computing center based on a massively parallel computer system and the transportation research and demonstration projects associated with key focus areas include the use of computing facilities as well as the exchange of research results with the private sector

and collaboration with universities to foster and encourage technology transfer. Current Argonne 
university partners include the University of Illinois, the Illinois Institute of Technology and Northern Illinois University.

\subsection{Operations, Computing, Networking, and Outreach Infrastructure, Facilities, and Capabilities}

TRACC expertise with videoconferencing and remote collaboration has led to a leading role at Argonne for the deployment of such technologies throughout the laboratory, in particular to reduce travel and

cut down both on costs and greenhouse gas emissions. This activity also significantly reduced the cost to TRACC for maintaining and even expanding this capability.

The Facility was heavily used to support the multiple monthly meetings of collaborators in the RTSTEP project as well as all the university projects that TRACC is involved in. TRACC also helped with the establishment of advanced communications technology for the new Emergency Management Operations Center at Argonne that was opened in late the summer 2011.

New options for acquiring a sufficient computing resource for future use by TRACC users were developed. The load on the current system is very high, while the infrastructure is aging rapidly. New specifications call for an architecture with many cores per node to better support multi-threaded shared memory applications, a trend in supercomputing that needs to be supported in the future. Acquiring a new system will also help with upgrading the existing system to a more modern operating system, something that cannot be easily accomplished when the machine is in heavy use.

Data on the HPC cluster's hard drive system is now backed up on a near-daily basis. A typical full backup requires approximately 10 days, and is performed from time to time. In between, daily differential backups are performed to ensure that data recovery is possible in case of the very unlikely failure of the highly redundant disk system.

\subsection{Transportation System Modeling and Evacuation Planning}

The RTSTEP Evacuation Modeling project for the City of Chicago started in mid-December 2010, and has made significant progress since then. The project is not funded by TRACC but rather through a grant from FEMA under the Regional Catastrophic Planning Grant Program (RCPGP) to the City of Chicago. Argonne has largely finished this work (by August 31 ) and is currently wrapping up the visualization and user interfaces, working with regional emergency planners to establish training courses, and working on documentation of the project. The normal activities of the transportation modeling group, such as training courses and user support, are continued throughout the RTSTEP implementation. A summary (as well as selected highlights) of the work on RTSTEP are reported in section 7 of this report.

The transportation systems modeling group is also preparing for work on a new multi-year project on the support of TRANSIMS and the development of new simulation approaches for the Office of Planning 
of the Federal Highway Administration. This project is expected to start at TRACC after the $1^{\text {st }}$ of October 2011 and will be reported on as part of this report.

The use of TRANSIMS on the cluster has steadily increased over the past year. This is due to a number of collaborators and independent cluster users ramping up parameter studies, and improved visualization capabilities that make it advisable to run TRANSIMS on the cluster.

\subsection{Computational Fluid Dynamics for Hydraulic and Aerodynamic Research}

Three model enhancements to the mesh morphing approach to scour simulation were completed during the quarter. The previous model displaced the bed only in the vertical direction, did not account for the effect of bed slope on critical shear stress, and allowed the slope of a scour hole to exceed the angle of repose of sand. The model now uses a variable critical shear stress that yields an increased critical shear stress when sediment particles are eroding from an uphill sloped bed and decreased critical shear stress when the particles are eroding from a downhill sloped bed. Bed displacements are now normal to the slope of the bed and a simple sand slide model is used to keep the eroded bed slope less than or equal

to the angle of repose of the particle bed. Modeling and analysis of the separation of flow at the leading edge of a flooded bridge deck was begun to aid in the development of an enhanced approach for evaluating scour due to submergence of bridge decks during floods in the federal guidelines. Modeling of flow through culverts for fish passage continued with the addition of a porous media model to capture the effects of large diameter gravel in the bottom of the culvert. TFHRC provided geometry files for their wind tunnel laboratory, and a new CFD model was developed to analyze flow in the wind tunnel and the room under a variety of flow conditions including with and without furniture. A CFD model using the sliding mesh capabilities of STAR-CCM+ with multiphase droplet tracking was developed and tested for application to the analysis of the interaction of road salt spray from large truck traffic with bridges using weathering steel beams.

\subsection{Multidimensional Visualization}

During this quarter, development continued on massive improvements of the TransimsVIS application. The TransimsVIS development was mostly performed as part of the RTSTEP project which is covered in more detail under the work for others section (section 7).

The visualizer is based on OpenGL and has been significantly improved in the past three months. The original concept as a data analysis tool for the post-processing of TRANSIMS results has been extended, and the visualizer is now able to run as part of the simulation process itself, as well as being able to create modifications of the models through an editing system that allows for a more efficient model building and optimization process. The software has been demonstrated at multiple occasions to emergency responders, and includes functionality to interactively define evacuation routes, evacuation areas, event scenarios, and population movements. 


\subsection{Computational Structural Mechanics Applications}

The Computational Structural Mechanics staff and Cluster Administrator continue to provide support resources to an expanding user base of DOT researchers using multi-physics computational structural mechanics tools to address design and safety issues. The workhorse software for multiphysics problems is LS-DYNA for which TRACC has 500 licenses. The average LS-DYNA usage for the second, third and fourth (current) quarters were, respectively, 190, 244 and 213 licenses. During all three quarters, the license usage was maxed out to the 500 licenses many times. The heaviest users of the LS-DYNA package during this quarter were Florida State University, NHTSA and TRACC with Northern Illinois University (NIU).

TRACC staff continued to provide support to and collaborate with Florida State University on paratransit bus safety research. This quarter's research shows how important the design of the frontal section of the bus structure is for crashworthy rollover behavior. A well design frontal ring is a critical component for a bus intended to pass a Florida Standard rollover test. For the USDOT, work continued on the study of blast load effects on stay cable bridges. A study was undertaken to evaluate several constitutive/failure models for reinforced concrete by comparing numerical predictions to small scale experiments reported in the open literature.

Research for Turner-Fairbank Highway Research Center - which is a Work for Others project - focused on vehicle stability under high crosswind loading conditions. This is a Master of Science thesis topic for a student at Northern Illinois University. Numerical simulations on a finite element model of an $8000 \mathrm{~S}$ vehicle (e.g., a Ford F800 single unit truck) subjected to several high wind conditions were performed. Based on vehicle speed and wind velocities, regions in which sideslip and rollover occur were identified. A second thesis topic was the development of an electromagnetic shock absorber control algorithm that can increase the stability of trucks driving in high wind conditions. Preliminary results show that the algorithm does offer an improvement in terms of reducing the acceleration of the sprung and unsprung masses, thus, potentially making the vehicle more stable in high winds. 


\section{Operations, Computing, Networking and Outreach Infrastructure, Facilities, and Capabilities}

In this area, the tasks identified in the current statement of work include:

- Operations

- Program Development Support

- High Performance Computing Cluster (user support, user software)

- TRACC Collaboratory - advanced facilities and communication in support of collaboration

TRACC and its programs are enabled by state-of-the-art equipment and facilities. The key infrastructure and facilities components of the TRACC project are the high-performance computational cluster and the high-bandwidth network connectivity provided at Argonne National Laboratory.

\subsection{TRACC Operations}

TRACC is currently in its fifth year of operation, and had been originally authorized under SAFETEA-LU in FY 2006 for a four year period. The U.S. Department of Transportation determined in June that TRACC had been sufficiently funded under the intent of the original congressional grant, and that new funds for its operation would no longer be provided. The existing FY 2010 funds at Argonne are sufficient to support operation throughout the current fiscal year (2012), and alternative funding sources are being pursued to ensure the continued operation beyond September 30, 2012. 


\subsection{Program Development Support}

TRACC is currently very active based on work funded from other sources than the SAFETEA-LU grant. This includes a major evacuation modeling project for the City of Chicago and advanced modeling and simulation work for the Turner Fairbank Highway Research Laboratory. A new major funding source from the Office of Planning of the Federal Highway Administration has been put in place to support TRACC's activities in the area of transportation systems modeling for the next two to three years. This project is starting shortly after October $1^{\text {st }}$, when the RTSTEP project with the City of Chicago is expected to end.

TRACC is also pursuing other activities, such as collaborations with universities and commercial partners to leverage the investment made into TRACC over the past five years. Outreach activities remain a strong component of TRACC's current activities, operating booths at trade shows and conferences, hosting summer students to work side by side with TRACC scientists on current projects, and supporting state and local organizations such as the Illinois Terrorism Task Force, the Illinois Department of Transportation, the Regional Catastrophic Preparedness Team for the Chicago Metropolitan Region, and others. This targets the application of state of the art capabilities to address urgent needs in evacuation planning and disaster response.

\subsection{High Performance Computing Cluster}

\subsubsection{User Support}

As demonstrated in the chapter on cluster usage and illustrated in the table below, the number of concurrent users has grown substantially in the past months. While some of the projects consuming large amounts of CPU time have scaled back, a larger number of smaller projects have taken their place, increasing the load on the cluster significantly.

Tape backups are now routinely performed to protect the data on the cluster and to provide users with a more reliable system. Preventive maintenance is now more important as the cluster hardware ages, and technical support contracts are being put in place to ensure the continued operation of the machine. Full backups are on a monthly schedule, and daily incremental backups are scheduled to be able to restore the entire system to a recent point in time.

Another issue is the update of the quite outdated operating system on the machine. The systems have of course been routinely updated to maintain the latest security patches, but the base versions of the operating systems are still RedHat version 4. The decision to delay major operating system updates had been made to provide a particularly stable production platform for users that would provide unchanged support for their application software. But RedHat version 4 is now at the end of its supported lifetime, and it becomes imperative to do a onetime major system upgrade to modernize the systems. This is being pursued at this time with spare parts in a test configuration. 
Based on the observed cluster load, plans were made for the acquisition of a similarly sized new cluster to ease the transition of users to a new operating system and to free up resources on the current machine to simplify the operating system upgrade. After putting these plans on hold initially when TRACC's funding base changed, a smaller upgrade is now being considered, and several different system designs are being evaulated

The following table has been revised and is now automatically generated from the user database. The format will be improved to allow for better statistical summaries and usage analysis. The past and current cluster users and user groups are shown in Table 2.1.

Table 2.1: Cluster Users

\section{3-D Numerical Simulation of Direct-Injection, Mixture Formation and Combustion in a} Hydrogen Engine

\begin{tabular}{|c|c|c|c|}
\hline & Energy Systems Divison, Argonne & & \\
\hline & Scarcelli & Riccardo & User \\
\hline & $\begin{array}{l}\text { U.S. DOE Engines and Emissions Control } \\
\text { Technologies }\end{array}$ & & \\
\hline & Singh & Gurpreet & Cognizant Engineer \\
\hline \multicolumn{4}{|c|}{ A Microstructure-Based Modeling Approach to Characterize Asphalt Materials } \\
\hline & $\begin{array}{l}\text { Michigan Department of } \\
\text { Transportation }\end{array}$ & & \\
\hline & Staton & John & Cognizant Engineer \\
\hline \multicolumn{4}{|c|}{ Michigan Technological University } \\
\hline & Barak & John & Cognizant Engineer \\
\hline & Colbert & Baron & User \\
\hline & Dai & Qingli & User \\
\hline & Lu & Haizhu & User \\
\hline & $N g$ & Kenny & User \\
\hline & You & Zhanping & User \\
\hline \multicolumn{4}{|c|}{ Analysis and Design of Roadside Safety Features for Safety Performance } \\
\hline \multicolumn{4}{|c|}{ Texas A\&M University } \\
\hline & Sheikh & N.M. & User \\
\hline \multicolumn{4}{|c|}{$\begin{array}{l}\text { Texas Transportation Institute, Texas } \\
\text { A\&M University }\end{array}$} \\
\hline & Bligh & Roger $P$. & Cognizant Engineer \\
\hline & Abu-Odeh & Akram Y. & User \\
\hline \multicolumn{4}{|c|}{ Application of Chaos Theory Analysis to Structural Health Monitoring of Cable-Stayed } \\
\hline
\end{tabular}




\begin{tabular}{|c|c|c|}
\hline $\begin{array}{l}\text { FHWA Nondestructive Evaluation } \\
\text { Center, TFHRC, USDOT }\end{array}$ & & \\
\hline Jalinoos & Frank & Cognizant Engineer \\
\hline Jin & Shuang & User \\
\hline Jin & Shuang & User \\
\hline \multicolumn{3}{|c|}{ Application of TRANSIMS for Highway Work Zones } \\
\hline FHWA Office of Planning, USDOT & & \\
\hline Yang & David & Cognizant Engineer \\
\hline Western Michigan University & & \\
\hline Oh & Jun-Seok & User \\
\hline \multicolumn{3}{|c|}{ Assessment Of Occupant Kinematics in Rollovers; Rollover Crash Analysis } \\
\hline $\begin{array}{l}\text { National Highway Traffic Safety } \\
\text { Administration Human Injury Division, } \\
\text { USDOT }\end{array}$ & & \\
\hline Ridella & Stephen & Cognizant Engineer \\
\hline Barsan-Anelli & Aida Cristina & User \\
\hline $\begin{array}{l}\text { Transportation Research and Analysis } \\
\text { Computing Center }\end{array}$ & & \\
\hline Bojanowski & Cezary & User \\
\hline \multicolumn{3}{|c|}{ Bridge Hydraulics - Analysis Support for Hydraulics Research in Transportation } \\
\hline $\begin{array}{l}\text { Transportation Research and Analysis } \\
\text { Computing Center }\end{array}$ & & \\
\hline Lottes & Steven A. & Cognizant Engineer \\
\hline Sofu & Tanju & Cognizant Engineer \\
\hline $\begin{array}{l}\text { Turner-Fairbank Highway Research } \\
\text { Center, FHWA, USDOT }\end{array}$ & & \\
\hline Kerenyi & Kornel & Cognizant Engineer \\
\hline Xie & Zhaoding & User \\
\hline Xie & Zhaoding & User \\
\hline University of Nebraska - Lincoln & & \\
\hline Guo & Junke & Cognizant Engineer \\
\hline Bushra & Afzal & User \\
\hline Bushra & Afzal & User \\
\hline Shan & Haoyin & User \\
\hline Shan & Haoyin & User \\
\hline Zhai & Yuan & User \\
\hline \multicolumn{3}{|l|}{ Bridge Scour Modeling Using CFD } \\
\hline California Department of Tranportation & & \\
\hline
\end{tabular}




\begin{tabular}{|c|c|c|}
\hline Flora & Kevin Scott & Cognizant Engineer \\
\hline \multicolumn{3}{|c|}{ Chicago Business District Evacuation (IDOT) } \\
\hline \multicolumn{3}{|l|}{ Northern Illinois University } \\
\hline$E$ & Manli & Developer \\
\hline Liu & Lichuan & Developer \\
\hline Ronanki & Bharani & Developer \\
\hline Pasupuleti & Lok Aradhya & User \\
\hline \multicolumn{3}{|l|}{ Nuclear Engineering Division, Argonne } \\
\hline Tentner & Adrian $M$. & User \\
\hline \multicolumn{3}{|c|}{ Chicago North Side Water Reclaimation Plant Studies } \\
\hline \multicolumn{3}{|l|}{$\begin{array}{l}\text { University of Illinois at Urbana- } \\
\text { Champaign }\end{array}$} \\
\hline Garcia & Marcelo & Cognizant Engineer \\
\hline Sinha & Sumit & User \\
\hline Tokyay & Talia Ekin & User \\
\hline \multicolumn{3}{|c|}{ Computational Mechanics Research and Support for Aerodynamics and Hydraulics at } \\
\hline TFHRC & & \\
\hline \multicolumn{3}{|l|}{ Northern Illinois University } \\
\hline Lanka Venkata & Sudhir Kumar & User \\
\hline Lanka Venkata & Sudhir Kumar & User \\
\hline \multicolumn{3}{|l|}{$\begin{array}{l}\text { Transportation Research and Analysis } \\
\text { Computing Center }\end{array}$} \\
\hline Lottes & Steven A. & Cognizant Engineer \\
\hline \multicolumn{3}{|c|}{$\begin{array}{l}\text { Computer Aided Mix Design (CAMiD) Technical and Analysis Support for Advanced } \\
\text { Computational Needs -- Bituminous Mixtures Laboratory at FHWA Turner-Fairbank } \\
\text { Research Center }\end{array}$} \\
\hline \multicolumn{3}{|l|}{ FHWA Office of Planning, USDOT } \\
\hline Gibson & Nelson & Cognizant Engineer \\
\hline \multicolumn{3}{|l|}{$\begin{array}{l}\text { Turner-Fairbank Highway Research } \\
\text { Center, FHWA, USDOT }\end{array}$} \\
\hline Kutay & Muhammed Emin & Cognizant Engineer \\
\hline \multicolumn{3}{|c|}{ Design-oriented Scoured Foundation Modeling for Bridge Performance Analysis } \\
\hline \multicolumn{3}{|l|}{ University of Missouri - Kansas City } \\
\hline Chen & ZhiQiang & User \\
\hline \multicolumn{3}{|c|}{ Development and Evaluation of Climate Metrics for Aviation based on Climate- } \\
\hline
\end{tabular}




\begin{tabular}{|c|c|c|}
\hline $\begin{array}{l}\text { Federal Aviation Administration, } \\
\text { USDOT }\end{array}$ & & \\
\hline Jacob & Daniel & Cognizant Engineer \\
\hline $\begin{array}{l}\text { University of Illinois at Urbana- } \\
\text { Champaign }\end{array}$ & & \\
\hline Wang & Dong & \\
\hline Lee & Huikyo & User \\
\hline Olsen & Seth & User \\
\hline Patten & Kenneth & User \\
\hline Wuebbles & Donald & User \\
\hline \multicolumn{3}{|c|}{ Development and Improvement of Roadside Safety Barriers } \\
\hline $\begin{array}{l}\text { Turner-Fairbank Highway Research } \\
\text { Center, FHWA, USDOT }\end{array}$ & & \\
\hline Opiela & Kenneth & Cognizant Engineer \\
\hline Arispe & Eduardo $N$. & User \\
\hline Eigen & Ana Maria & User \\
\hline \multicolumn{3}{|c|}{ Development of TRANSIMS Models to Evaluate Traffic Management Plans for Special } \\
\hline Illinois Institute of Technology & & \\
\hline Li & Zongzhi & Cognizant Engineer \\
\hline Lee & Sang Hyuk & User \\
\hline Lee & YongDoo & User \\
\hline Lee & YongDoo & User \\
\hline Rahman & Asadur & User \\
\hline Rahman & Asadur & User \\
\hline Veliou & Eirini & User \\
\hline Veliou & Eirini & User \\
\hline \multicolumn{3}{|c|}{ Development of TRANSIMS software for use in Parallelization of TRANSIMS } \\
\hline Yelchuru & Balaji & User \\
\hline AECOM & & \\
\hline Roden & David & User \\
\hline Argonne National Laboratory & & \\
\hline Toonen & Brian $R$. & User \\
\hline U.S. Department of Transportation & & \\
\hline Gardner & Brian & Cognizant Engineer \\
\hline
\end{tabular}




\begin{tabular}{|c|c|c|}
\hline Leonard & John & Cognizant Engineer \\
\hline Georgia Institute of Technology & & \\
\hline Guin & Angshuman & User \\
\hline Shealey & Stephanie Lynne & User \\
\hline $\begin{array}{l}\text { Transportation Research and Analysis } \\
\text { Computing Center }\end{array}$ & & \\
\hline Ley & Hubert & Cognizant Engineer \\
\hline \multicolumn{3}{|c|}{ Development of TRANSIMS Visualization Software Metropolis } \\
\hline $\begin{array}{l}\text { National Center for Supercomputing } \\
\text { Applications, University of Illinois }\end{array}$ & & \\
\hline Cox & Donna & Cognizant Engineer \\
\hline Betts & Alex & User \\
\hline Hall & Matthew & User \\
\hline Levy & Stuart & User \\
\hline Patterson & Robert & User \\
\hline $\begin{array}{l}\text { Transportation Research and Analysis } \\
\text { Computing Center }\end{array}$ & & \\
\hline Ley & Hubert & Cognizant Engineer \\
\hline \multicolumn{3}{|l|}{ Electromagnetic Shock Absorber } \\
\hline Northern Illinois University & & \\
\hline Fisher & Francisco Lucas & User \\
\hline \multicolumn{3}{|c|}{ Evaluation of Thor Dummy FE Model in Crash Environments } \\
\hline $\begin{array}{l}\text { National Highway Traffic Safety } \\
\text { Administration Human Injury Division, } \\
\text { USDOT }\end{array}$ & & \\
\hline Ridella & Stephen & Cognizant Engineer \\
\hline University of Virginia & & \\
\hline Untaroi & Costin & User \\
\hline \multicolumn{3}{|c|}{ FHWA Signal Processing, Data Analysis and Computational Modeling for Highway } \\
\hline $\begin{array}{l}\text { Turner-Fairbank Highway Research } \\
\text { Center, FHWA, USDOT }\end{array}$ & & \\
\hline Cobb & Lincoln & Cognizant Engineer \\
\hline Scott & Michael & Cognizant Engineer \\
\hline Chintakunta & Satish Reddy & User \\
\hline Gagarin & $N$. & User \\
\hline Mekemson & James Robert & User \\
\hline
\end{tabular}




\begin{tabular}{|c|c|c|}
\hline \multicolumn{3}{|c|}{$\begin{array}{l}\text { Geosynthetic-Reinforced Soil Integral Bridge System; Scour Counterr } \\
\text { Deformation Analysis of Bridge Supported on Shallow Foundations }\end{array}$} \\
\hline FHWA Office of Planning, USDOT & & \\
\hline Hartmann & Joey & Cognizant Engineer \\
\hline Nicks & Jennifer & User \\
\hline \multicolumn{3}{|c|}{ Improvements of LS-Dyna Models for Off-Road Traffic Accident Analysis } \\
\hline Kineticorp & & \\
\hline Carter & Neal & User \\
\hline Rose & Nathan & User \\
\hline Zhu & Ling & User \\
\hline \multicolumn{3}{|c|}{ Inner Nozzle Flow characterization of Biodiesel from Different Feedstocks } \\
\hline Argonne National Laboratory & & \\
\hline Som & Sibendu & User \\
\hline $\begin{array}{l}\text { Transportation Research and Analysis } \\
\text { Computing Center }\end{array}$ & & \\
\hline Lottes & Steven A. & Cognizant Engineer \\
\hline $\begin{array}{lrrr}\text { U.S. DOE } & \text { Energy } & \text { Efficiency \& } \\
\text { Renewable } & \text { Energy } & - & \text { Vehicle } \\
\text { Technologies } & \text { Program } & \\
\end{array}$ & & \\
\hline Stork & Kevin & Cognizant Engineer \\
\hline \multicolumn{3}{|l|}{ In-situ Load Rating of Highway Bridges } \\
\hline University of Virginia & & \\
\hline Chase & Robert Paul & User \\
\hline Jin & Shuang & User \\
\hline $\operatorname{Jin}$ & Shuang & User \\
\hline \multicolumn{3}{|c|}{ Investigation of Bridge Pier and Abutment Scour Using Large Eddy Simulation } \\
\hline University of lowa & & \\
\hline Constantinescu & Serban George & Cognizant Engineer \\
\hline Dunn & Mark & Cognizant Engineer \\
\hline Miyawaki & Shinjiro & User \\
\hline Tokyay & Talia Ekin & User \\
\hline \multicolumn{3}{|c|}{$\begin{array}{l}\text { Investigation of impact factors for FDOT bridges; and the best practices in construction } \\
\text { of paratransit buses }\end{array}$} \\
\hline FAMU-FSU College of Engineering & & \\
\hline Gepner & Bronislaw Dominik & User \\
\hline Kwasniewski & Leslaw & User \\
\hline
\end{tabular}




\begin{tabular}{|c|c|c|}
\hline Siervogel & Jeff & User \\
\hline Taft Ford & Eduardo $E$. & User \\
\hline Wekezer & Jerry & User \\
\hline Florida Department of Transportation & & \\
\hline Westbrook & Robert & Cognizant Engineer \\
\hline \multicolumn{3}{|c|}{$\begin{array}{l}\text { Investigation of River Flow and Transport Processes Using High Resolution Eddy } \\
\text { Resolving CFD Simulations }\end{array}$} \\
\hline $\begin{array}{l}\text { South Florida Water Management } \\
\text { District }\end{array}$ & & \\
\hline Zeng & Jie & User \\
\hline University of lowa & & \\
\hline Constantinescu & Serban George & Cognizant Engineer \\
\hline Chang & Kyoungsik & User \\
\hline Kashyap & Shalini & User \\
\hline Steenhauer & Kate & User \\
\hline Suhodolova & Tatiana & User \\
\hline \multicolumn{3}{|c|}{ Large Eddy Simulation of Interaction of Turbulent Flow with a Porous Media } \\
\hline $\begin{array}{l}\text { Transportation Research and Analysis } \\
\text { Computing Center }\end{array}$ & & \\
\hline Lottes & Steven A. & Cognizant Engineer \\
\hline $\begin{array}{l}\text { University of Illinois at Urbana- } \\
\text { Champaign }\end{array}$ & & \\
\hline Samala & Rahul & Cognizant Engineer \\
\hline Vanka & Pratap & Cognizant Engineer \\
\hline \multicolumn{3}{|l|}{ Management } \\
\hline Energy Systems Divison, Argonne & & \\
\hline Drucker & Harvey & Manager \\
\hline $\begin{array}{l}\text { Transportation Research and Analysis } \\
\text { Computing Center }\end{array}$ & & \\
\hline Ley & Hubert & Manager \\
\hline Tate & Gail & Manager \\
\hline Weber & David & Manager \\
\hline \multicolumn{3}{|c|}{$\begin{array}{l}\text { Modeling Hot Mix Asphalt Compaction - Technical Support for Pavement Design and } \\
\text { Performance Modeling Team }\end{array}$} \\
\hline Delft University of Technology & & \\
\hline Casbergen & c. & User \\
\hline Jonsthovel & Tom Bernard & User \\
\hline
\end{tabular}




\begin{tabular}{|c|c|c|}
\hline Koneru & Saradhi & User \\
\hline Liu & $x$. & User \\
\hline Skarpas & Athanasios & User \\
\hline $\begin{array}{l}\text { FHWA Nondestructive Evaluation } \\
\text { Center, TFHRC, USDOT }\end{array}$ & & \\
\hline Weaver & Eric & Cognizant Engineer \\
\hline Kasbergen & Cornelis & User \\
\hline Texas A\&M University & & \\
\hline Masad & Eyad & User \\
\hline Scarpas & Tom & User \\
\hline \multicolumn{3}{|c|}{ Moreno Valley TRANSIMS Transportation Modeling/ITS } \\
\hline City of Moreno Valley & & \\
\hline Kerenyi & John & Cognizant Engineer \\
\hline Gross & Mark & User \\
\hline Keller & James & User \\
\hline Lloyd & Michael David & User \\
\hline Minard & Mark & User \\
\hline Van Simaeys Jr & Julien & User \\
\hline FHWA Office of Planning, USDOT & & \\
\hline Gardner & Brian & Cognizant Engineer \\
\hline \multicolumn{3}{|c|}{ MOVES (Motor Vehicle Emission Simulator), Modeling emissions from on road vehicles } \\
\hline $\begin{array}{l}\text { U.S. Environmental Protection Agency, } \\
\text { Office of Transportation and Air } \\
\text { Qualilty }\end{array}$ & & \\
\hline Beardsley & Megan & \\
\hline Aikman & William Russell & Cognizant Engineer \\
\hline Michaels & Harvey & Cognizant Engineer \\
\hline Brzezinski & David & User \\
\hline Choi & Jongwoo David & User \\
\hline Church & Thomas Michael & User \\
\hline Faler & Wesley Gordon & User \\
\hline Glover & $E d$ & User \\
\hline Kahan & Ari & User \\
\hline Maciag & Ted & User \\
\hline Shyu & Gwo Ching & User \\
\hline \multicolumn{3}{|l|}{ NHTSA Human Injury Research Division } \\
\hline $\begin{array}{l}\text { National Highway Traffic Safety } \\
\text { Administration Human Injury Division, }\end{array}$ & & \\
\hline
\end{tabular}




\begin{tabular}{|c|c|c|}
\hline USDOT & & \\
\hline Takhounts & Erik & Cognizant Engineer \\
\hline $\begin{array}{l}\text { National Highway Traffic Safety } \\
\text { Administration, USDOT }\end{array}$ & & \\
\hline Parent & Daniel & User \\
\hline \multicolumn{3}{|c|}{ Nonlinear Analysis of Cable-Stayed Bridge Cables } \\
\hline Northern Illinois University & & \\
\hline Gupta & Abhijit & User \\
\hline Vannemreddi & S. & User \\
\hline Vannemreddy & B. & User \\
\hline $\begin{array}{l}\text { Transportation Research and Analysis } \\
\text { Computing Center }\end{array}$ & & \\
\hline Kulak & Ronald F. & Cognizant Engineer \\
\hline \multicolumn{3}{|c|}{ Numerical Investigation of Single Point Incremental Forming } \\
\hline Northwestern University & & \\
\hline Cao & Jian & Cognizant Engineer \\
\hline Huang & Ying & User \\
\hline \multicolumn{3}{|c|}{ Open Channel Flow, Bridge Hydraulics, and Bridge Scour } \\
\hline Northern Illinois University & & \\
\hline Kostic & Milivoje & Cognizant Engineer \\
\hline Majumdar & Pradip & Cognizant Engineer \\
\hline Biswas & Dipankar & User \\
\hline Edwards & Christopher M. & User \\
\hline Elapolu & Phani Ganesh & User \\
\hline Pati & Vishnu Vardhan Reddy & User \\
\hline Tulimilli & Bhaskar Rao & User \\
\hline $\begin{array}{l}\text { Transportation Research and Analysis } \\
\text { Computing Center }\end{array}$ & & \\
\hline Lottes & Steven A. & Cognizant Engineer \\
\hline \multicolumn{3}{|c|}{ RTSTEP: Regional Transportation Simulation Tool for Evacuation Planning } \\
\hline AECOM & & \\
\hline Gregerson & Christopher & User \\
\hline Patnam & Krishna Chandra & User \\
\hline Patnam & Krishna Chandra & User \\
\hline Patnam & Krishna Chandra & User \\
\hline Roden & David & User \\
\hline Santhanam & Srividya & User \\
\hline Singuluri & Sashank & User \\
\hline
\end{tabular}




\begin{tabular}{|c|c|c|}
\hline Chicago Department of Transportation & & \\
\hline Emmanuel & Abraham & Advisor \\
\hline Illinois Institute of Technology & & \\
\hline Arthur & Christina & User \\
\hline Liu & $Y i$ & User \\
\hline Liu & Yifang & User \\
\hline Lu & $x i$ & User \\
\hline$Q u$ & Fei & User \\
\hline Yi & Bo & User \\
\hline Zhong & Yaoqian & User \\
\hline Zhou & Bei & User \\
\hline Northern Illinois University & & \\
\hline Moraga & Reinaldo J. & User \\
\hline Tanaka & Raul Augusto Alvarez & User \\
\hline Zhang & Quan & User \\
\hline $\begin{array}{l}\text { Office of Emergency Management, Cirt } \\
\text { of Chicago }\end{array}$ & & \\
\hline Halac & Yilmaz & Sponsor \\
\hline $\begin{array}{l}\text { Transportation Research and Analysis } \\
\text { Computing Center }\end{array}$ & & \\
\hline Sokolov & Vadim 0. & Cognizant Engineer \\
\hline Auld & Joshua & Developer \\
\hline Hope & Michael & Developer \\
\hline Zhang & Kuilin & Developer \\
\hline Holifield & Justin & User \\
\hline Kang & Sherry & User \\
\hline Park & Young Soo & User \\
\hline Qualls & Timothy & User \\
\hline \multicolumn{3}{|l|}{ SACOG TRANSIMS Implementation } \\
\hline Resource Systems Group, Inc & & \\
\hline Bowman & John L. & User \\
\hline Bradley & Mark Andrew & User \\
\hline Castiglione & Joseph & User \\
\hline Lawe & Stephen & User \\
\hline Rentz & Erich & User \\
\hline U.S. Department of Transportation & & \\
\hline Gardner & Brian & Cognizant Engineer \\
\hline Grady & Brian Robert & Cognizant Engineer \\
\hline
\end{tabular}




\begin{tabular}{|c|c|c|}
\hline \multicolumn{3}{|l|}{ SIMon Brain Model } \\
\hline $\begin{array}{l}\text { National Highway Traffic Safety } \\
\text { Administration Human Injury Division, } \\
\text { USDOT }\end{array}$ & & \\
\hline Ridella & Stephen & Cognizant Engineer \\
\hline Takhounts & Erik & Cognizant Engineer \\
\hline U.S. Department of Transportation & & \\
\hline Hasija & Vikas & User \\
\hline \multicolumn{3}{|l|}{ Snowdrift Mitigation } \\
\hline \multicolumn{3}{|l|}{ University of lowa } \\
\hline Constantinescu & Serban George & Cognizant Engineer \\
\hline Basnet & Keshav & User \\
\hline \multicolumn{3}{|l|}{ Software Testing } \\
\hline \multicolumn{3}{|l|}{ CD-adapco } \\
\hline Reynolds & Robert & Vendor \\
\hline Schnepper & Carol & Vendor \\
\hline \multicolumn{3}{|l|}{ Nuclear Engineering Division, Argonne } \\
\hline Thomas & Justin W. & User \\
\hline \multicolumn{3}{|c|}{ SToRM-Cluster; Computation River Hydraulics and Transfer } \\
\hline \multicolumn{3}{|l|}{ U.S. Geological Survey } \\
\hline Simoes & Francisco J.M. & Cognizant Engineer \\
\hline \multicolumn{3}{|c|}{ Structural Performance of Flexible Pavements } \\
\hline \multicolumn{3}{|l|}{\begin{tabular}{|ll|l}
$\begin{array}{l}\text { Louisiana Transportation Research } \\
\text { Center }\end{array}$ & \\
\end{tabular}} \\
\hline Zhang & Zhao & \\
\hline Chen & Xingwei & User \\
\hline \multicolumn{3}{|c|}{$\begin{array}{l}\text { Structural Testing Laboratory Computational Mechanics Research; Finite Element } \\
\text { Modeling of Highway Structures }\end{array}$} \\
\hline \multicolumn{3}{|l|}{$\begin{array}{l}\text { Turner-Fairbank Highway Research } \\
\text { Center, FHWA, USDOT }\end{array}$} \\
\hline Beshah & Fassil & Cognizant Engineer \\
\hline Adams & Michael & User \\
\hline Chen & Linfeng & User \\
\hline Graybeal & Ben & User \\
\hline Greene & Gary & User \\
\hline Ocel & Justin & User \\
\hline Study of Erosion Mechanisms at Piers & f Rectangula & solving \\
\hline
\end{tabular}




\begin{tabular}{|c|c|c|}
\hline \multicolumn{3}{|c|}{ Techniques and use of the Databases for Improvement of RANS Scour Models } \\
\hline University of lowa & & \\
\hline Constantinescu & Serban George & Cognizant Engineer \\
\hline Kerenyi & Kornel & Cognizant Engineer \\
\hline Cheng & Zhengyang & User \\
\hline Koken & Mete & User \\
\hline Yuksel Ozan & Ayse & User \\
\hline \multicolumn{3}{|c|}{ Study of the Rate of Deterioration of Bridges and Pavements as Affected by Trucks } \\
\hline Clemson University & & \\
\hline Amirkhanian & Serji & Cognizant Engineer \\
\hline Swanka & Merrill & Cognizant Engineer \\
\hline Chowdhary & Ronnie & User \\
\hline Dunning & Anne & User \\
\hline Pang & Wei Chiang & User \\
\hline Putman & Brad & User \\
\hline \multicolumn{3}{|l|}{ Systems Administation } \\
\hline $\begin{array}{l}\text { Computing and Information Systems, } \\
\text { Argonne }\end{array}$ & & \\
\hline DiGiacomo & Denice & SysAdmin \\
\hline Domagala & Paul & SysAdmin \\
\hline Dust & James & SysAdmin \\
\hline Hall & Corey & SysAdmin \\
\hline Hilden & Michael & SysAdmin \\
\hline Schmitz & Corby B. & SysAdmin \\
\hline Winkler & Linda & SysAdmin \\
\hline $\begin{array}{l}\text { Mathematics and Computer Science } \\
\text { Division, Argonne }\end{array}$ & & \\
\hline Valdes & John P. & SysAdmin \\
\hline $\begin{array}{l}\text { Transportation Research and Analysis } \\
\text { Computing Center }\end{array}$ & & \\
\hline Amiot & Lawrence W. & SysAdmin \\
\hline Bernard & Jon & SysAdmin \\
\hline Kolpacki & Penny & SysAdmin \\
\hline Mihalo & William E. & SysAdmin \\
\hline Nowakowski & Waldemar & SysAdmin \\
\hline Reitzer & Joe & SysAdmin \\
\hline Schmitt & Robert & SysAdmin \\
\hline
\end{tabular}




\begin{tabular}{|c|c|c|}
\hline Northern Illinois University & & \\
\hline Gupta & Abhijit & Cognizant Engineer \\
\hline Balpande & Rohit Suresh & User \\
\hline \multicolumn{3}{|c|}{$\begin{array}{l}\text { Traffic Simulation of St. Louis Transportation Network for Earthquake Impact } \\
\text { Assessment }\end{array}$} \\
\hline Newmark Civil Engineering Laboratory & & \\
\hline Elnashai & Amr $S$. & Cognizant Engineer \\
\hline Spencer & William $F$. & Cognizant Engineer \\
\hline $\begin{array}{l}\text { University of Illinois at Urbana- } \\
\text { Champaign }\end{array}$ & & \\
\hline Chang & Liang & User \\
\hline \multicolumn{3}{|c|}{ TRANSIMS Configuration Control, Testing, Automation, and Software Release } \\
\hline Northern Illinois University & & \\
\hline Malar & $\kappa$ & Developer \\
\hline U.S. Department of Transportation & & \\
\hline Ducca & Fred & Cognizant Engineer \\
\hline Gardner & Brian & Cognizant Engineer \\
\hline \multicolumn{3}{|c|}{ TRANSIMS Evacuation Traffic Models of New Orleans and Houston } \\
\hline Louisiana State University & & \\
\hline Wolshon & Brian & Cognizant Engineer \\
\hline Dixit & Vinayak & User \\
\hline Montz & Thomas & User \\
\hline Zhang & Zongjie & User \\
\hline \multicolumn{3}{|c|}{$\begin{array}{l}\text { TRANSIMS Technology and Tools development technical assistance for the } \\
\text { development Buffalo USA/Canadian Region cross-border model. }\end{array}$} \\
\hline U.S. Department of Transportation & & \\
\hline Smith & Scott & Cognizant Engineer \\
\hline Chase & Robert Paul & User \\
\hline Mekuria & Maaza & User \\
\hline \multicolumn{3}{|c|}{ TRANSIMS Traffic Simulation Software Development/Parallelization } \\
\hline Northern Illinois University & & \\
\hline Kanaga-Nayagam & Kanesa & User \\
\hline $\begin{array}{l}\text { Transportation Research and Analysis } \\
\text { Computing Center }\end{array}$ & & \\
\hline Ley & Hubert & Cognizant Engineer \\
\hline Hope & Michael & User \\
\hline
\end{tabular}




\begin{tabular}{|c|c|c|}
\hline \multicolumn{3}{|l|}{ Unspecified } \\
\hline Argonne National Laboratory & & \\
\hline Faber & Merlyn & \\
\hline Nguyen & Vu & \\
\hline Pinkerton & Scott & \\
\hline $\begin{array}{l}\text { Arizona State University, Department } \\
\text { of Transporation }\end{array}$ & & \\
\hline Volosin & Sarah Elia & \\
\hline Pendyala & Ram & User \\
\hline Plotz & Joseph & User \\
\hline Chrysler & & \\
\hline Zhu & Yuhua & \\
\hline $\begin{array}{l}\text { Mathematics and Computer Science } \\
\text { Division, Argonne }\end{array}$ & & \\
\hline Kim & Jong $G$. & \\
\hline Medical College of Wisconsin & & \\
\hline Hallman & Jason & User \\
\hline Michigan Technological University & & \\
\hline Stanton & Brett & \\
\hline Northern Illinois University & & \\
\hline Kuppa & Lakshmi Pratyusha & User \\
\hline $\begin{array}{l}\text { South Carolina Department of } \\
\text { Transportation }\end{array}$ & & \\
\hline Zwanka & Merrill & \\
\hline U.S. Department of Transportation & & \\
\hline Rudd & Rodney W & \\
\hline \multicolumn{3}{|c|}{ Update LA DOTD Policy on Pile Driving Vibration Management } \\
\hline $\begin{array}{l}\text { Louisiana Transportation Research } \\
\text { Center }\end{array}$ & & \\
\hline Gautreau & Garvin & Cognizant Engineer \\
\hline Worcester Polytechnic Institute & & \\
\hline Tao & Mingjiang & Cognizant Engineer \\
\hline Zhang & Mo & User \\
\hline \multicolumn{3}{|c|}{$\begin{array}{l}\text { Using TRANSIMS to Quantify the Impacts of Planned Transportation Projects on } \\
\text { Congestion and Air Quality }\end{array}$} \\
\hline $\begin{array}{lll}\begin{array}{l}\text { Georgia } \\
\text { Authority }\end{array} & \text { Regional Transportation } \\
\end{array}$ & & \\
\hline Goodwin & Robert & Cognizant Engineer \\
\hline
\end{tabular}




\begin{tabular}{|l|l|l|l|}
\hline & Pihl & Eric & Cognizant Engineer \\
\hline & Davis & Curt & User \\
\hline & Rousseau & Guy & User \\
\hline & Zuehlke & Kai & User \\
\hline \multicolumn{2}{|l|}{ Vehicle Stability under High Winds } & \multicolumn{2}{l|}{} \\
\hline & Northern Illinois University & Rohan Satish & User \\
\hline & Patil & \multicolumn{2}{|l|}{} \\
\hline Wind Loads on Highway Sign and Traffic Signal Structures & Cognizant Engineer \\
\hline & University of lowa & Serban George & User \\
\hline & Constantinescu & Shinjiro & User \\
\hline & Miyawaki & Talia Ekin & \\
\hline & Tokyay
\end{tabular}

\subsubsection{Usage Statistics}

Usage statistics were updated to include the entire history of operation, giving a complete picture of overall TRACC cluster usage. For the $4^{\text {th }}$ quarter (July through September 2011), the cluster has been heavily used.

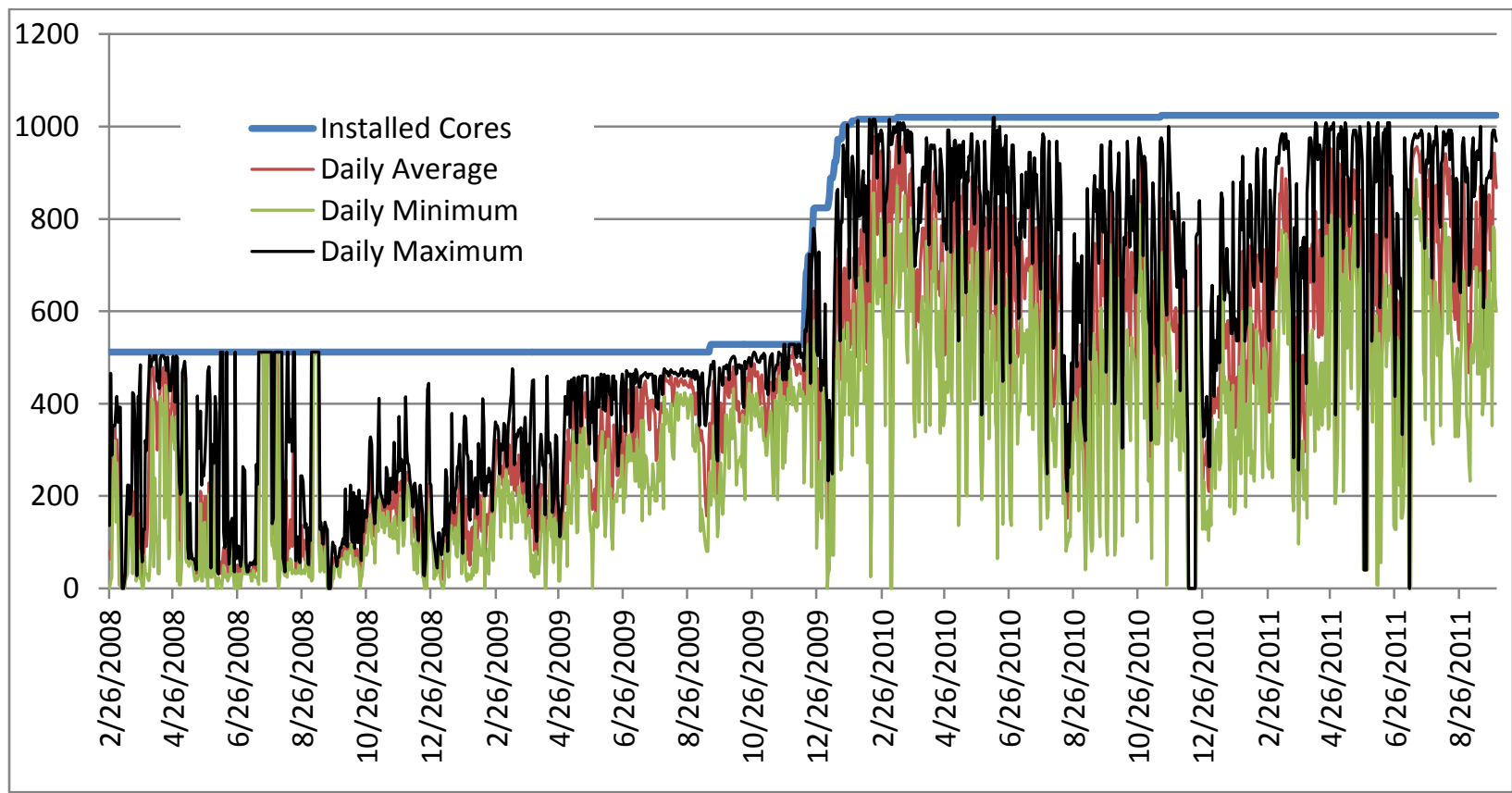

Figure 2.1: Daily Cluster Node Usage with Min and Max Number of Nodes since 2008

The graph clearly shows that the usage of the cluster is bound by the available hardware, and an upgrade of the existing hardware is an important plan for this fiscal year. 


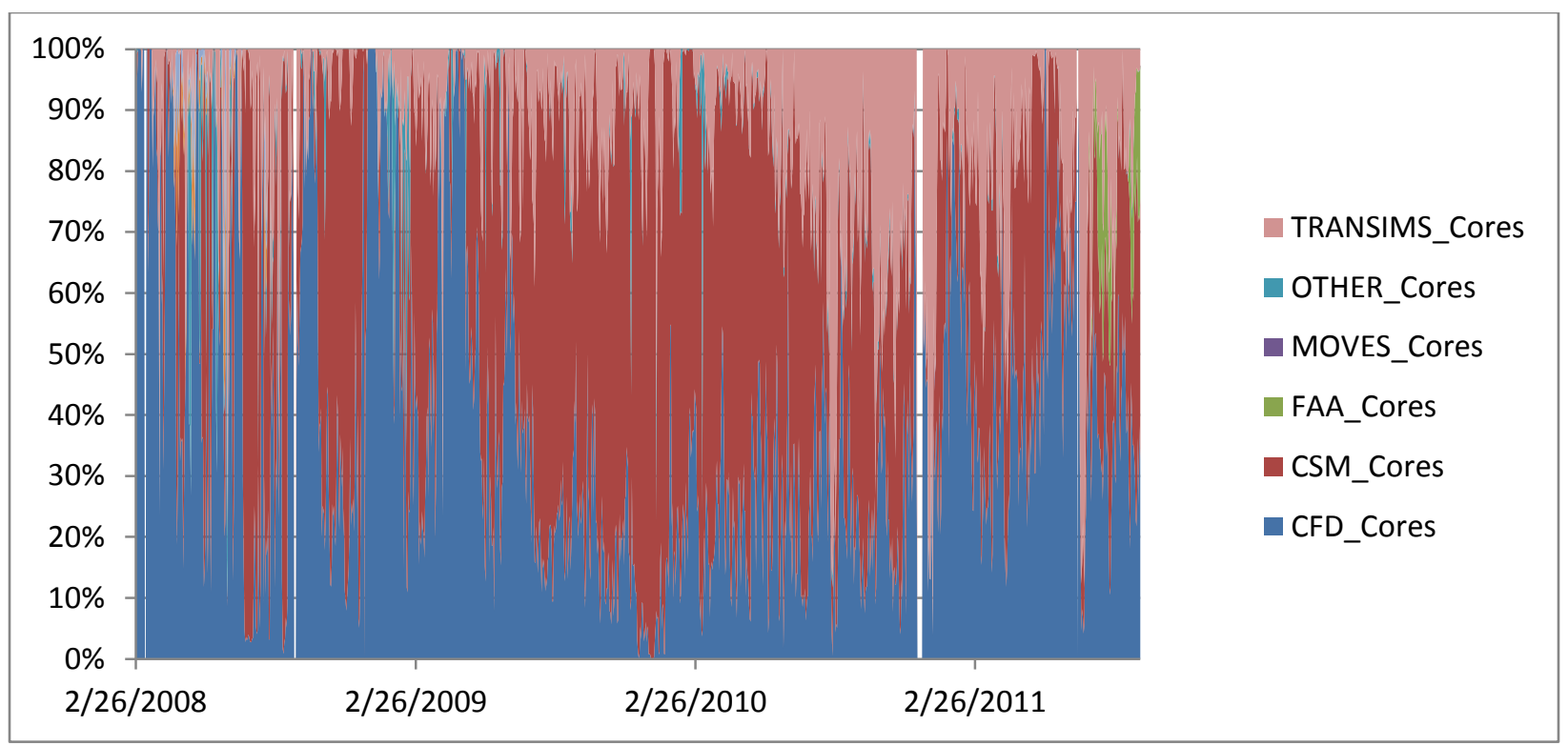

Figure 2.2: Relative Daily Cluster Node Usage by Application Area

Figure 2.6 illustrates the relative breakdown of cluster usage by application area. The newly created accounts for FAA users can be found on the right of the graph, marked in green. Usage in the structural mechanics area has gone down somewhat due to several heavy users (graduate students at the time) finishing their work. The EPA (using the MOVES application) did not put a significant load onto the machine, although they had estimated that they may need up to $25 \%$ of TRACC capacity for several months in the spring. Computation fluid dynamics codes and TRANSIMS made up for the remainder of the load. Additional applications are expected to start up taking significant load in the coming months

More detailed statistics will be provided with the next quarterly report, including year end statistics.

\subsubsection{User Software}

TRACC is running GCC Version 4.5.2 as a loadable module on the cluster. Two different versions of Star$\mathrm{CCM}+$ were placed into service and are being provided to users. An updated version of OpenFOAM is now supported on the cluster. The major software applications currently running on the TRACC cluster are shown in Table 2.2. 
Table 2.2: Major Software Applications currently available on the TRACC cluster

\begin{tabular}{|c|c|}
\hline \multicolumn{2}{|c|}{ Traffic Simulation and Evacuation Planning } \\
\hline TRANSIMS & https://wiki.anl.gov/tracc/TRANSIMS \\
\hline \multicolumn{2}{|c|}{ Computational Fluid Dynamics } \\
\hline STAR-CD ${ }^{\circledast}$ & https://wiki.anl.gov/tracc/STAR-CD \\
\hline $\mathrm{STAR}-\mathrm{CCM}+{ }^{\circledR}$ & https://wiki.anl.gov/tracc/STAR-CCM+ \\
\hline STAR-Design $^{\circledR}$ & https://wiki.anl.gov/tracc/STAR-Design \\
\hline ANSYS FLUENT $^{\oplus}$ & https://wiki.anl.gov/tracc/Fluent \\
\hline 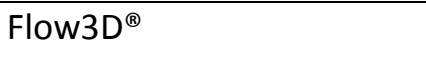 & https://wiki.anl.gov/tracc/Flow3D \\
\hline OpenFOAM & http://www.opencfd.co.uk/index.html \\
\hline \multicolumn{2}{|c|}{ Computational Structural Mechanics } \\
\hline LS-DYNA $^{\circledR}$ & https://wiki.anl.gov/tracc/LS-DYNA \\
\hline LS-OPT $^{\circledR}$ & https://wiki.anl.gov/tracc/LS-OPT \\
\hline Madymo ${ }^{\circledR}$ & https://wiki.anl.gov/tracc/MADYMO \\
\hline ModeFrontier & https://wiki.anl.gov/tracc/ModeFRONTIER \\
\hline \multicolumn{2}{|c|}{ Mesh Generators, Toolkits, Utilities, Compilers } \\
\hline NoMachine & $\begin{array}{l}\text { https://wiki.anl.gov/trac/Using the cluster\# } \\
\text { Graphical Applications with NoMachine }\end{array}$ \\
\hline Hypermesh & $\begin{array}{l}\text { http://www.altairhyperworks.com/Product,7,HyperMesh.aspx } \\
\text { https://wiki.anl.gov/tracc/Hyperworks }\end{array}$ \\
\hline Hyperview & $\begin{array}{l}\text { http://www.altairhyperworks.com/Product,7,HyperMesh.aspx } \\
\text { https://wiki.anl.gov/tracc/Hyperworks }\end{array}$ \\
\hline LS-PrePost & $\begin{array}{l}\text { http://www.Istc.com/lspp/ } \\
\text { https://wiki.anl.gov/tracc/LS-PREPOST }\end{array}$ \\
\hline Salome-Platform & https://wiki.anl.gov/tracc/SALOME \\
\hline MATLAB $^{\circledR}$ & https://wiki.anl.gov/tracc/MATLAB \\
\hline Intel C++ and Fortran & https://wiki.anl.gov/tracc/Compilers \\
\hline GNU C++ and Fortran & https://wiki.anl.gov/tracc/Compilers \\
\hline Pathscale $\mathrm{C}++$ and Fortran & https://wiki.anl.gov/tracc/Compilers \\
\hline Intel MPI libraries & https://wiki.anl.gov/tracc/MPI libraries \\
\hline
\end{tabular}




\section{Transportation Systems Modeling and Evacuation Planning}

Tasks for Transportation Systems Modeling and Evacuation planning in this quarter include:

- Technical Support for TRANSIMS

- TRANSIMS Improvements and Further Development of the Chicago Metropolitan Model

- TRANSIMS Outreach, User Support, Training and Technology Enablement

A significant amount of work has been done in this area. The work was performed under the RTSTEP project contract and is reported in the "Work for Others" section 7 of this report. The RTSTEP project is a \$2M project involving about 25 collaborators at Northern Illinois University, the Illinois Institute of Technology, the Chicago Metropolitan Agency for Planning, the City of Chicago, and AECOM, a large consulting and engineering company. TRACC is managing the project and has hired three additional staff members. 7 staff members at TRACC are working on this project now, and the work is closely related to work performed for USDOT in the past. The project was largely finished in August 2011, and due to the constraints on personnel at TRACC, most activities in the transportation modeling section were temporarily funded out of this project.

TRANSIMS has become a significant part of the work at TRACC, and as demonstrated in the chapter on cluster usage, TRANSIMS has steadily increased the load on the TRACC cluster. Several projects operated solely by TRACC cluster users are making massive use of the machine, and TRACC had to provide frequent support to run jobs effectively and to fix small bugs in the underlying software. 


\section{Computational Fluid Dynamics for Hydraulic and Aerodynamic Research}

Tasks for Computational Fluid Dynamics for Hydraulic and Aerodynamic Research in the fifth year at TRACC

- Technical Support of TRACC External Users for CFD Applications and Software

- Technical Contributions to FHWA Bridge Hydraulics Analysis Program

- Outreach, User Support, Training and Technology Enablement

\subsection{Technical Support of TRACC External Users in CFD Applications and Software}

The TRACC staff continue to support external users with issues related to the use of the TRACC cluster, the software installed on the cluster, and user research software that has been ported to the cluster. Installation of the latest version of the open source CFD software OpenFOAM, 2.0.1, encountered a number of difficulties related to the version of Red Hat Linux on the cluster. Most of these issues have been resolved and there is a serial version of 2.0.1 available. There is still an issue in compiling the latest version of OpenFOAM for parallel use with the versions of the parallel message passing interface (MPI) libraries available on the cluster, and resolution of this problem is being pursued. Support was provided to the University of lowa to resolve problems with running a large eddy simulation (LES) research code that had been ported to the TRACC cluster and was working two years ago. A new post-doctoral researcher was beginning work with this software and a number of issues related to compiling the software and running it in the system environment on the TRACC cluster were resolved with the help of TRACC cluster administrators. The University of lowa LES research software is now functioning again, and a grateful thank you email was received from the team leader. 


\subsection{Technical Contributions to the FHWA Bridge Hydraulics Analysis Program}

The effort during the fourth quarter was focused on completing enhancements to the cylindrical bridge scour models that have been developed in prior work. The previous model displaced the bed only in the vertical direction, did not account for the effect of bed slope on critical shear stress, and allowed the slope of a scour hole to exceed the angle of repose of sand. Model enhancements to address all of these issues were completed, and the details are presented in Section 4.3.

\subsection{Three Dimensional Iterative Scouring Methodology Using Commercial CFD Software}

Major progress has been made in developing and implementing enhancements to the scour model that address issues limiting the original scour methodology: bed slope effects on shear stress, vertical-only scour, and scour hole walls having slopes larger than the angle of repose, $\phi$, of the sediment particles. Work on improved models to address these issues was completed and is documented in detail in [3]. An overview of the new models and some of the results of applying them to modeling cylindrical pier scour are given in the following sections.

\subsubsection{Computational Domain, Volume Mesh and Boundary Conditions}

Simulations are run using STAR-CCM+ version 6.04. The simulations are implicit unsteady using a varying time step and employ a 2-layer realizable k-epsilon turbulence model. A half flume is modeled by placing a symmetry plane along the center of the flume, running in the direction of flow, to save computational time. 


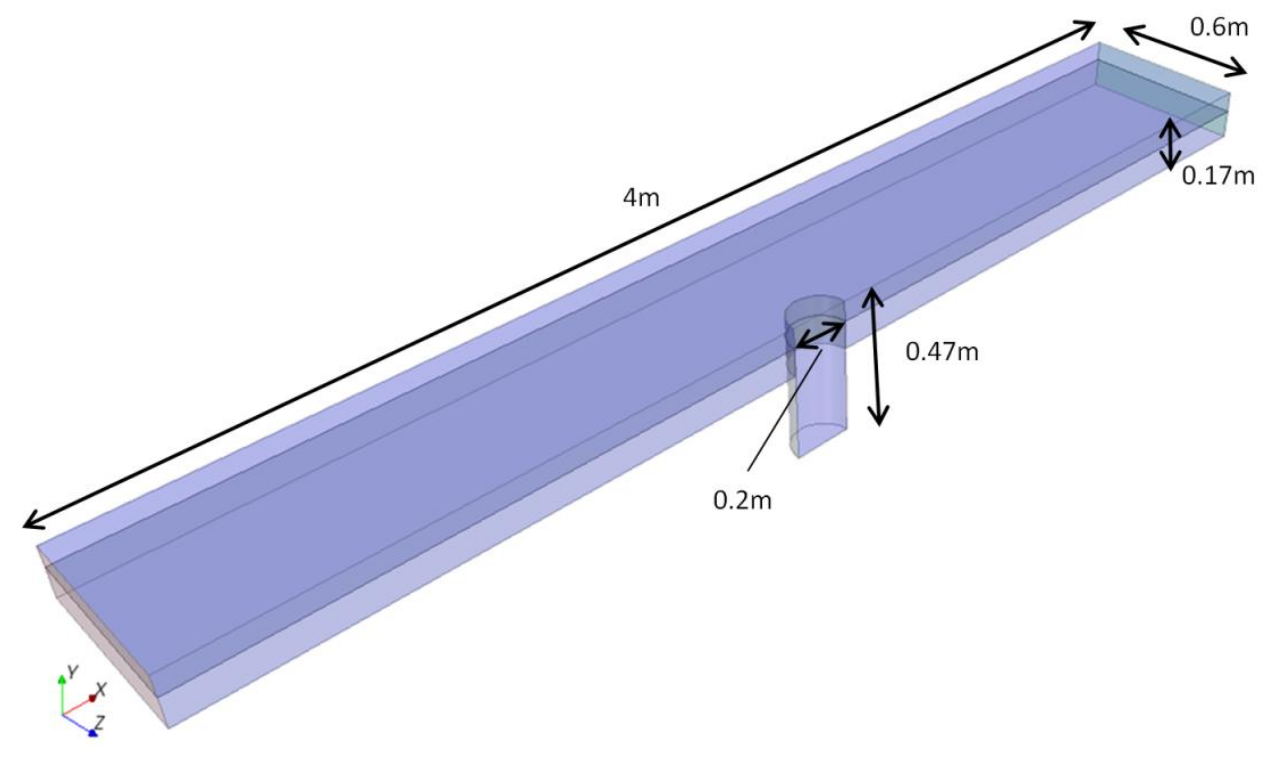

Figure 4.1: Computational domain with key dimensions labeled

The computational domain extends from $-2 \mathrm{~m}$ to $+2 \mathrm{~m}$ in the primary flow or X-direction, $-0.6 \mathrm{~m}$ to $0 \mathrm{~m}$ in the cross stream or Z-direction and $0 \mathrm{~m}$ to $0.17 \mathrm{~m}$ in the vertical or Y-direction. A solid, fixed cylinder is included in the computational domain and is cut along the symmetry plane in the same manner as the flume. The cylinder has a radius of $0.1 \mathrm{~m}$ in the XZ-plane, has a vertical height of $0.47 \mathrm{~m}$, and runs from the top of the flume to $0.3 \mathrm{~m}$ below the initial sediment bed height. The cylinder serves mostly as a marking post to visually inspect the amount of scour in relation to water depth and is meshed with a minimum number of cells. The domain is divided into two regions along the height of the cylinder in order to ensure that mesh morphing occurs only in the bottom region to save computational time and prevent a previously encountered problem of negative volume cells being generated at the top surface after mesh morphing.

The computational domain is meshed using a core cell size of $20 \mathrm{~mm}$. A refined mesh is placed around the cylinder using a cell size of $5 \mathrm{~mm}$. Also, a prism layer is used at the bed boundary to maintain the correct cell thickness for the application of turbulent wall functions (satisfying the $y+$ wall requirement). The near-wall prism layer thickness is specified as $5 \mathrm{~mm}$. The solid cylinder is also meshed so that a welldefined interface between the solid and fluid cylinder walls can be created.. The initial volume mesh contains approximately 265,000 cells and the number slowly increases as the sediment bed is eroded, increasing its volume, and remeshed to maintain high mesh quality. 


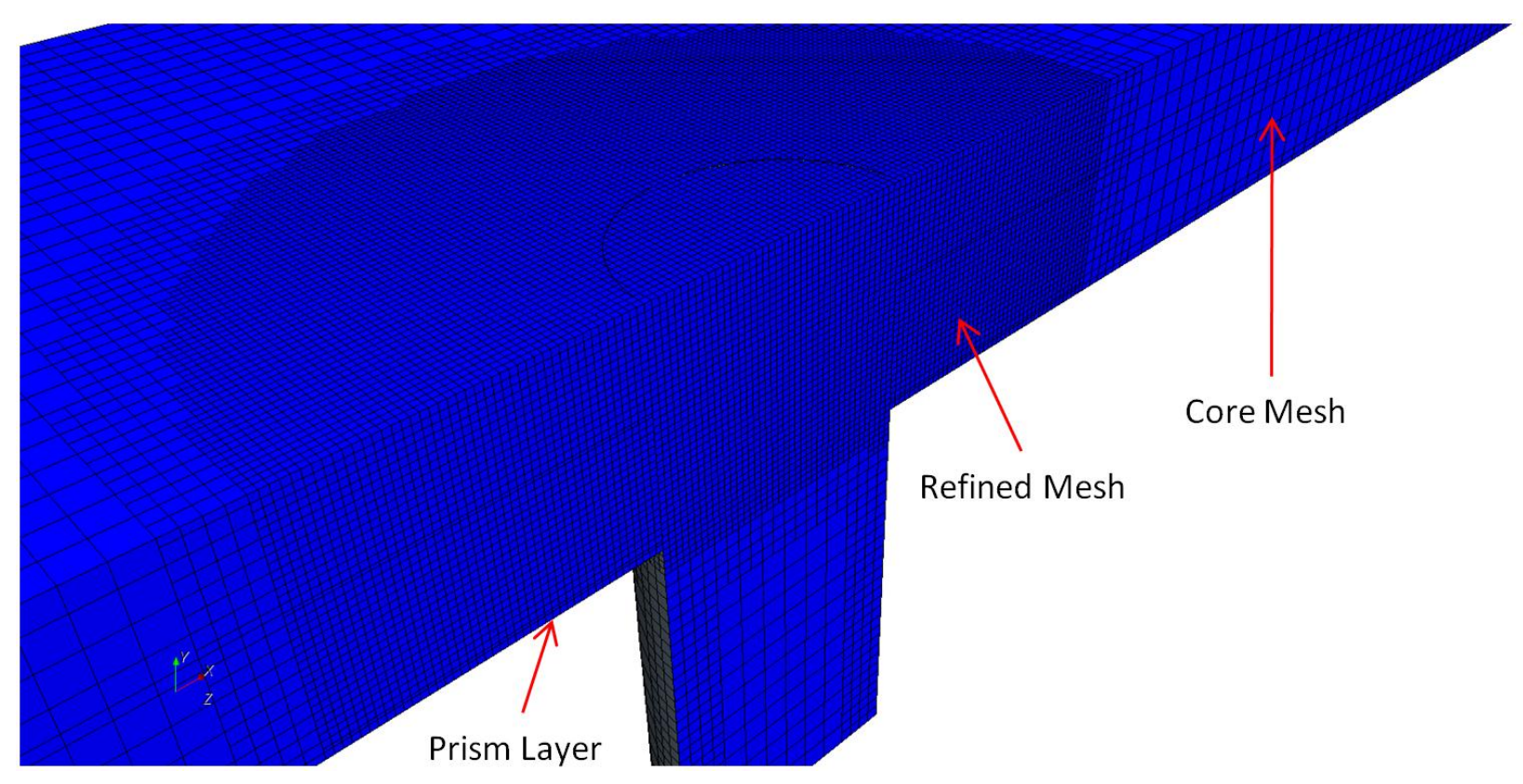

Figure 4.2: Volume mesh of computational domain used in simulations

There are two distinct regions in the flow domain: a solid region and a fluid region. The solid region consists only of a vertical cylinder while the fluid region consists of two domains that comprise the flume. Each boundary in the computational domain has a different set of associated parameters. Symmetry boundaries do not have mesh morphing conditions because mesh points in a symmetry plane are not moved in the mesh morphing process. All other boundaries have a fixed mesh morphing condition except for the sediment bed and the cylinder bottom in the fluid region. The bed boundary has a grid velocity mesh morphing condition to allow the sediment bed to move during the scour process, while the cylinder bottom has a floating mesh morphing condition which allows the cylinder to follow the shape of the bed as it is scoured. The inlet has a specified fluid velocity of $0.5 \mathrm{~m} / \mathrm{s}$ in the Xdirection. The outlet has a well-developed outflow boundary condition, which allows the fluid to exit the computational domain as if the flume continued after the outlet.

\subsubsection{Mesh Morphing}

The simulation of scouring and formation of a scour hole is done through a mesh morphing process in STAR-CCM+. In the mesh morphing process a grid velocity, represented by a vector, is used to move the sediment bed at the beginning of each time step. The grid velocity, or erosion rate, is determined from shear stress at the sediment bed using an entrainment rate function from the literature, Van Rijn[1]. During mesh morphing, as the sediment bed moves downward, not just the layer of cells adjacent to the sediment bed are moved downward; rather, the displacement is distributed by stretching the cells throughout the specified region. This helps maintain a high quality mesh. If the morphed mesh cells become overly stretched due to the formation of deep scour holes, the situation is remedied by remeshing the computational domain periodically during the computation. 


\subsubsection{Grid Velocity and Time Step Equations}

The grid velocity is the rate of displacement of the bed due to erosion and is calculated at the beginning of each time step. The grid velocity is derived from a sediment pickup function from Van Rijn [1]:

$$
d h /_{d t}=\frac{0.00033 \sqrt{\Delta g d_{50}} d_{*}^{0.3}}{\theta_{s}}\left(\frac{\tau_{b e d}}{\tau_{c}}-1\right)^{1.5}
$$

where

$$
\begin{gathered}
\Delta=\left(\rho_{s}-\rho\right) / \rho \\
d_{*}=d_{50}\left[\frac{\Delta g}{v^{2}}\right]^{1 / 3} \\
\theta_{s}=1-\gamma
\end{gathered}
$$

Where $\rho_{s}$ and $\rho$ are the densities of the sediment and the water respectively, $\tau_{c} \tau_{\mathrm{c}}$ is the critical shear stress value, $\tau_{\text {bed }}$ is the shear stress at a given point on the bed, $g$ is the acceleration due to gravity, $\mathrm{d}_{50} d_{50}$ is the mean sand diameter, $\theta_{s}$ is the packing density, $\gamma$ is the porosity of the sand, $d h / d t$ is the grid velocity of the sediment bed and $v$ is the kinematic viscosity of water.

The grid velocity is used to determine a variable time step. The time step is varied in order to keep the maximum amount of scour in any time step small to maintain numerical stability. Currently, the maximum bed displacement is set equal to $0.25 \mathrm{~mm}$. This has an effect of making time steps very small, approximately 0.05 seconds, in the early stages of scouring and very large, greater than 60 seconds, as the simulation approaches convergence. Using a small maximum bed displacement has the effect of making the flow nearly quasi-steady during the period of a time step. The quasi-steady nature of the flow on the erosion time scale allows the computation with the implicit solver to remain stable as the erosion rate slows and time steps become large. Using a time step that is inversely proportional to the erosion rate allows a computation that would take months of wall time to be completed in less than two weeks. The length of each time step is determined based on the maximum specified displacement and grid velocity, given by the following equation

$$
\Delta t=\frac{\Delta \mathrm{y}_{\max }}{\max (d h / d t)}
$$

where $\Delta y_{\max }$ is the user specified maximum displacement for each time step and $\Delta t$ is the length of each time step. The local bed displacement at each point on the bed is obtained from Equation 4.6. The bed is displaced at the beginning of each time step in the mesh morphing process. 


$$
\Delta y=(d h / d t) \Delta t
$$

\subsubsection{Variable Critical Shear Stress (VCSS) Approach}

The previous methodology used a constant critical shear stress (CCSS) value in grid velocity calculations. CCSS is based upon flat bed shear stress, which has a value of $0.18 \mathrm{~Pa}$ for $0.24 \mathrm{~mm}$ sand, and is unrealistic on the sloped bed regions that form during the scouring process. A methodology that uses a variable critical shear stress (VCSS) has been developed and implemented in the scour model. The VCSS value is a function of the bed slope in the direction of the shear stress on the bed. The VCSS approach can be used in conjunction with other scouring model improvements (scouring normal to the bed and modeling sand slides when the bed slope exceeds the angle of repose) to move the sediment bed during simulations.

The variable critical shear stress is formulated using a correction factor from Bohrs [2] applied to the flat bed critical shear stress to obtain the critical shear stress on a sloping bed. This relation has the form:

$$
\tau_{c}=\frac{\sin (\gamma+\phi)}{\sin (\phi)} \tau_{c f b}
$$

where $\tau_{c f b}$ is the flat bed critical shear stress and $\phi$ is the angle of repose of the sand, taken to be 33 degrees. In Bohrs, $\gamma$ is stated to be the slope of the bed. Taking $\gamma$ as the angle of the bed with respect to the horizontal direction is fine for two dimensional scour where the bed shear is either directly downslope or directly upslope, however, in general 3D cases, the bed slope will not be parallel to the direction of flow or the bed shear. Therefore, in the model developed here, $\gamma$ is taken to be the angle of the bed in the direction of the shear stress with respect to the horizontal. In this case, $\gamma$ is given by

$$
\gamma=\cos ^{-1}\left(\frac{\tau_{\text {bed }} \cdot g}{\left\|\tau_{\text {bed }}\right\|\|g\|}\right)-\frac{\pi}{2}
$$

where $\gamma$ is limited to be in the range $-\phi<\gamma<\phi$. Outside of this range, a sand slide model should be applied to reduce the bed slope to within the angle of repose.

\subsubsection{Normal Scour Approach}

Another approach to improve the original methodology is to include scouring perpendicular, or normal, to the sediment bed at each computational cell face on the bed where the shear stress is greater than the critical shear stress. The original methodology moves the sediment bed only in the vertical direction, which is reasonable when the bed slope is small, such as in pressure flow scour, but not for pier scour. Scouring normal to the bed uses the grid velocity, Equation 4.1, in conjunction with the sediment bed normal vector to move each face. Equation 4.9 defines how the grid velocity (erosion rate vector, $E_{r}$ ) is calculated using the normal scour approach. 


$$
\overrightarrow{E_{r}}=(d h / d t) \frac{\vec{A}}{\|A\|}
$$

The last term in Equation 4.9 is the bed cell face area vector divided by its magnitude, which is a unit vector normal to the cell face. Figure 4.3 illustrates the difference between vertical scouring and scouring normal to the bed.

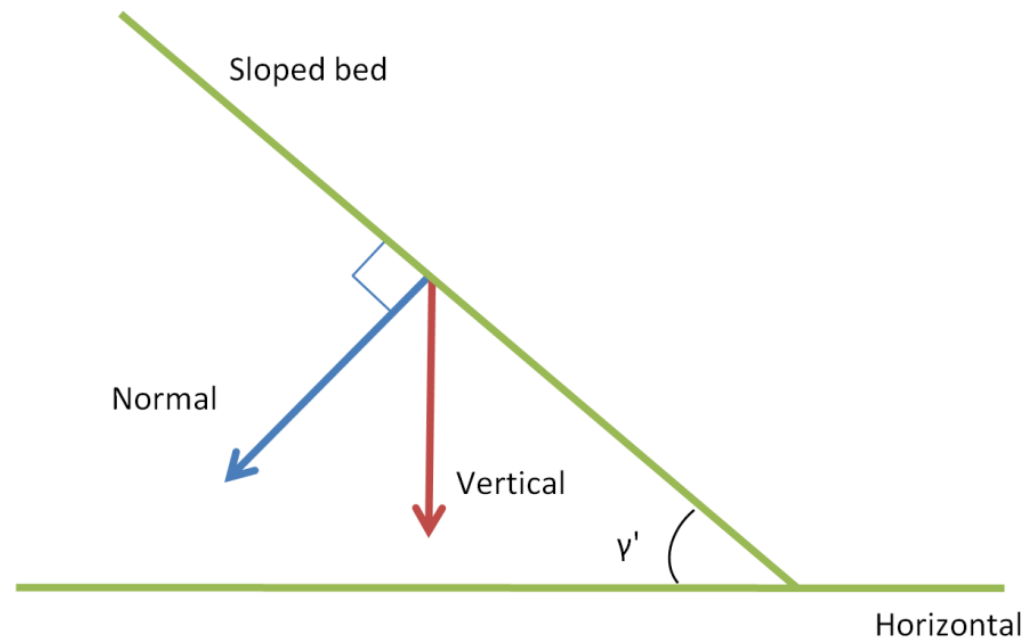

Figure 4.3: Schematic of normal scour approach showing re ssentative sediment bed cell face

At the end of every time step before bed displacement and mesh morphing, the erosion rate vector, $\boldsymbol{E}_{\boldsymbol{r}}$, is split into components to perform the displacement normal to each bed cell face that is undergoing scour.

\subsubsection{Sand Slide Approach}

Another method to reduce the slope of scour hole walls is to incorporate a sand slide model into the scouring methodology. The sand slide model that is currently being developed only takes into account the angle of the sediment bed cell face and does not calculate stresses inside the sediment bed. This approach will reduce the slope of scour hole walls to the angle of repose, while not having the computational overhead of calculating stresses inside the sediment bed.

\subsubsection{Field Functions for Scour Physics Models}

The field functions used in STAR-CCM+ to implement the scour model using mesh morphing with a variable time step including the functions used in the three model enhancements covered in this report are given in Table 4.1. 
Table 4.1 STAR-CCM+ field functions for scour physics models

\begin{tabular}{|c|c|c|}
\hline Name & Definition & Description \\
\hline Alpha & $\operatorname{acos}(\operatorname{dot}(\$ \$$ UnitA, unit(\$\$ravity))) & $\begin{array}{l}\text { Angle of sediment bed for } \\
\text { use in sand slides (rad) }\end{array}$ \\
\hline VCritShear & $\$ F B C S S *(\sin (\$ P h i+\$ G a m m a) / \sin (\$ P h i))$ & $\begin{array}{l}\text { VCSS value before floor } \\
\text { function is applied }(\mathrm{Pa})\end{array}$ \\
\hline D50 & 0.00025 & Median sand diameter $(\mathrm{m})$ \\
\hline Delta & (\$DenSand-\$DenWater)/\$DenWater & Term used in grid velocity \\
\hline DeltaT & 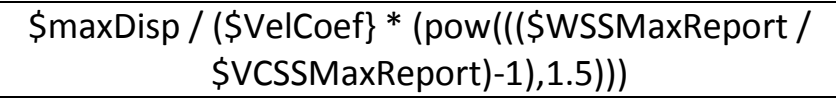 & Variable time step (s) \\
\hline DenSand & 2650 & Density of sand $\left(\mathrm{kg} / \mathrm{m}^{3}\right)$ \\
\hline DenWater & 1000 & Density of water $\left(\mathrm{kg} / \mathrm{m}^{3}\right)$ \\
\hline Dstar & $\begin{array}{c}\text { \$D50*pow }(((\$ \text { Delta * }(- \\
\text { \$\$Gravity[1]))/pow }(\$ N u, 2)),(1 / 3))\end{array}$ & Dimensionless diameter \\
\hline FBCSS & 0.18 & $\begin{array}{l}\text { Flat bed critical shear stress } \\
(\mathrm{Pa})\end{array}$ \\
\hline Gamma & $\operatorname{acos}(\operatorname{dot}($ unit $(\$$ tauWS), unit(\$\$Gravity))) - (\$Pi/2) & $\begin{array}{l}\text { Angle of the bed in the } \\
\text { direction of the shear stress } \\
\text { with respect to the } \\
\text { horizontal (rad) }\end{array}$ \\
\hline Gravity & {$[0,-9.81,0]$} & Gravity vector $\left(\mathrm{m} / \mathrm{s}^{2}\right)$ \\
\hline GridVelLimiter & $\begin{array}{c}\text { ( } \$ \text { DeltaT * \$GridVel > \$maxDisp) ? \$GridVel == } \\
(\text { \$maxDisp } / \text { \$DeltaT }): \$ \text { GridVel }\end{array}$ & $\begin{array}{l}\text { Ensures grid velocity does } \\
\text { not displace more than } \\
\text { specified amount per time } \\
\text { step }\end{array}$ \\
\hline GridVel & $\begin{array}{c}(\$ \$ \text { Centroid }[0]>-1.5) \quad ?(\operatorname{mag}(\$ \$ \text { tauWS })> \\
\text { \$VCSS) ? \$VelCoeff* } \\
\text { pow }(((\operatorname{mag}(\$ \$ \text { tauWS }) / \$ V C S S)-1), 1.5): 0: 0\end{array}$ & $\begin{array}{l}\text { Non-constant term in grid } \\
\text { velocity equation }(\mathrm{m} / \mathrm{s})\end{array}$ \\
\hline maxDisp & 0.00025 & $\begin{array}{l}\text { Maximum bed displacement } \\
\text { per time step }(\mathrm{m})\end{array}$ \\
\hline $\mathrm{Nu}$ & $8.887 e-7$ & Kinematic Viscosity $\left(\mathrm{m}^{2} / \mathrm{s}\right)$ \\
\hline NormalBC & 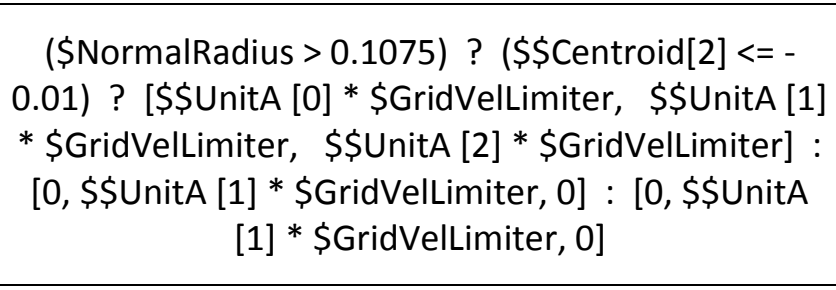 & $\begin{array}{l}\text { Changes normal scouring to } \\
\text { vertical near bridge pier and } \\
\text { centerline symmetry plane } \\
\text { boundaries. Used to avoid } \\
\text { scouring the pier and } \\
\text { symmetry plane }(\mathrm{m} / \mathrm{s})\end{array}$ \\
\hline NormalRadius & $\operatorname{sqrt}(\operatorname{pow}(\$ \$$ Centroid$[0], 2)+\operatorname{pow}(\$ \$$ Centroid $[2], 2))$ & $\begin{array}{l}\text { Distance from center of pier } \\
\text { to cell centroid }(\mathrm{m})\end{array}$ \\
\hline NormScour & $\begin{array}{c}(\$ \text { Centroid }[0]>-1.5 \& \& \$ \$ \text { Centroid }[0]<1.75) ? \\
(\$ A \text { Alpha }<(\$ \mathrm{Pi} / 2)) ?(\operatorname{mag}(\$ \$ \text { tauWS })>\$ V C S S) ? \\
\$ \$ \text { NormalBC }:[0,0,0]:[0,0,0]:[0,0,0]\end{array}$ & $\begin{array}{l}\text { Normal scour rate used in } \\
\text { grid velocity }(\mathrm{m} / \mathrm{s})\end{array}$ \\
\hline Phi & $33 *(\$ \mathrm{Pi} / 180)$ & $\begin{array}{l}\text { Angle of repose of sand } \\
\text { (rad) }\end{array}$ \\
\hline $\mathrm{Pi}$ & 3.14159265358979 & Pi to double precision \\
\hline PorosityS & 0.43 & Sand porosity \\
\hline
\end{tabular}




\begin{tabular}{|c|c|c|}
\hline Name & Definition & Description \\
\hline SandSlide & 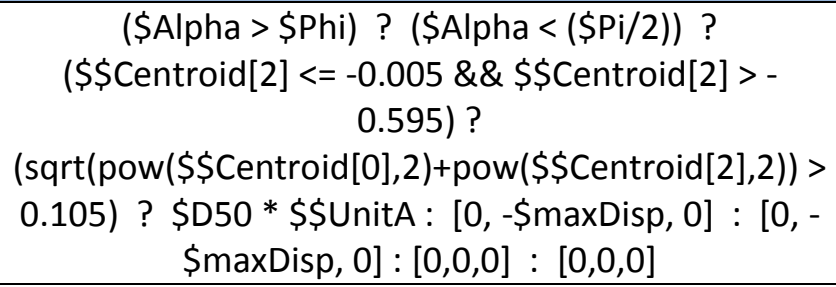 & $\begin{array}{l}\text { Sand slide displacement (m). } \\
\text { Includes special conditions } \\
\text { at boundaries }\end{array}$ \\
\hline sigmaW & $\operatorname{dot}(\$ \$$ WallShearStress, $\$ \$$ UnitA $) * \$ \$$ UnitA & $\begin{array}{l}\text { Wall shear stress vector } \\
\text { projected onto bed unit } \\
\text { normal vector }(\mathrm{Pa})\end{array}$ \\
\hline tauWs & \$WallShearStress - \$\$sigmaW & $\begin{array}{l}\text { Wall shear stress vector } \\
\text { parallel to bed cell faces }(\mathrm{Pa})\end{array}$ \\
\hline UnitA & unit(\$Area) & $\begin{array}{l}\text { Area unit normal vector for } \\
\text { sediment bed cells }\end{array}$ \\
\hline VCSS & $\begin{array}{c}\text { (\$VCritShear > } \\
\text { \$VCSSFloor) ? \$VCritShear: } \\
\text { \$VCSSFloor }\end{array}$ & $\begin{array}{l}\text { Relation for VCSS with floor } \\
\text { function included }(\mathrm{Pa})\end{array}$ \\
\hline VCSSFloor & $0.1 * \$ F B C S S$ & VCSS floor function (Pa) \\
\hline VelCoeff & $\begin{array}{l}(0.00033 * \text { pow }(\$ D \text { Dstar,0.3) * pow((\$Delta*(- } \\
\text { \$Gravity[1])*\$D50),(1/2))) / (1-\$PorosityS) }\end{array}$ & $\begin{array}{l}\text { Constant term in grid } \\
\text { velocity equation }(\mathrm{m} / \mathrm{s})\end{array}$ \\
\hline
\end{tabular}

\subsubsection{Simulation Results}

Results using all three of the model enhancements are presented in this section. Two dimensional plots that are generated on a slice through the domain or one dimensional plots generated from data taken along a line on the bed use the plane or line positions shown in Figure 4.4. The other surface used for plotting results is the sediment bed with the initial bed shown in the figure. Note that the center of the pier is located at the origin, $[0,0,0]$, in the coordinate system.

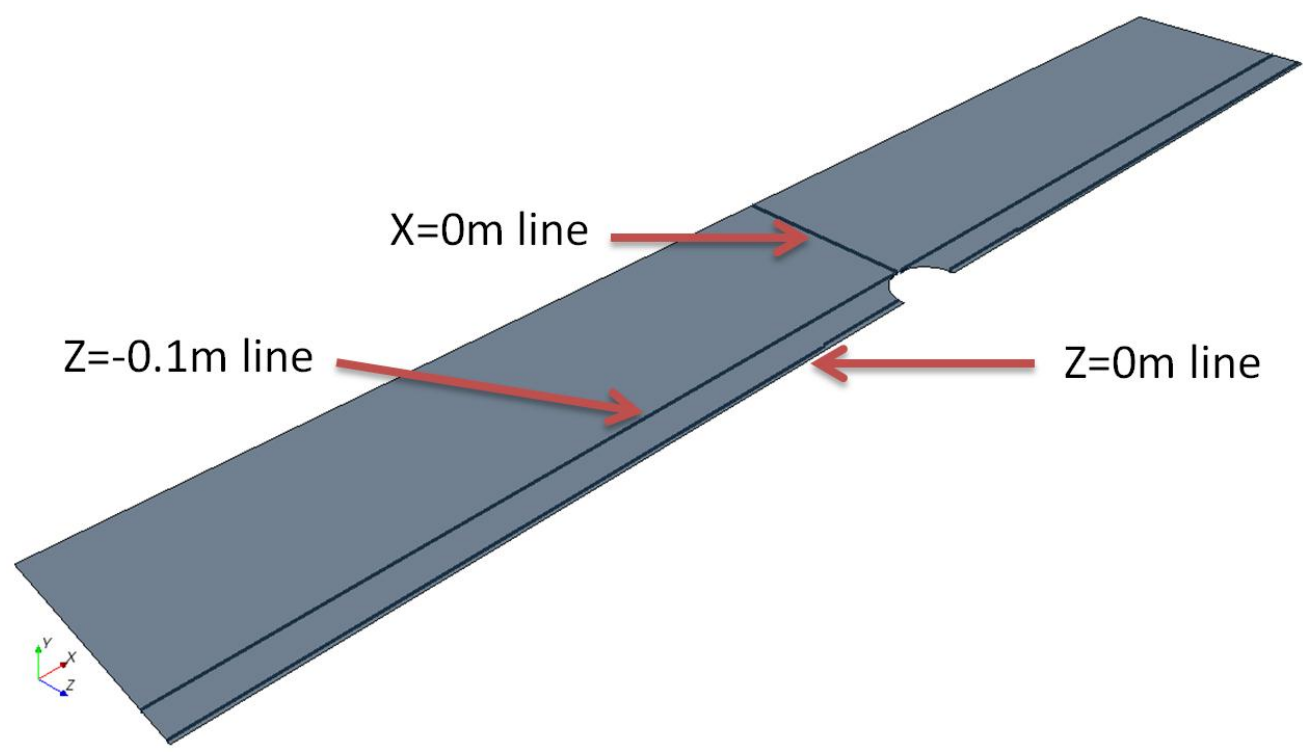


Figure 4.4: Initial bed geometry showing positions used to obtain data for plotted results.

The automated procedure for periodically remeshing and computing sand slides during the scour computation currently takes more than a week of wall time to complete on the TRACC cluster. Efforts to optimize for running time have currently been limited to running on an optimum number of cluster nodes. The time to converge a time step during early time steps is shown as a function of the number of cluster compute nodes used in Figure 4.5. The computational mesh contained 265,000 cells. For this problem size, there is no significant reduction in solver time beyond using 3 compute nodes for a total of 24 processor cores.

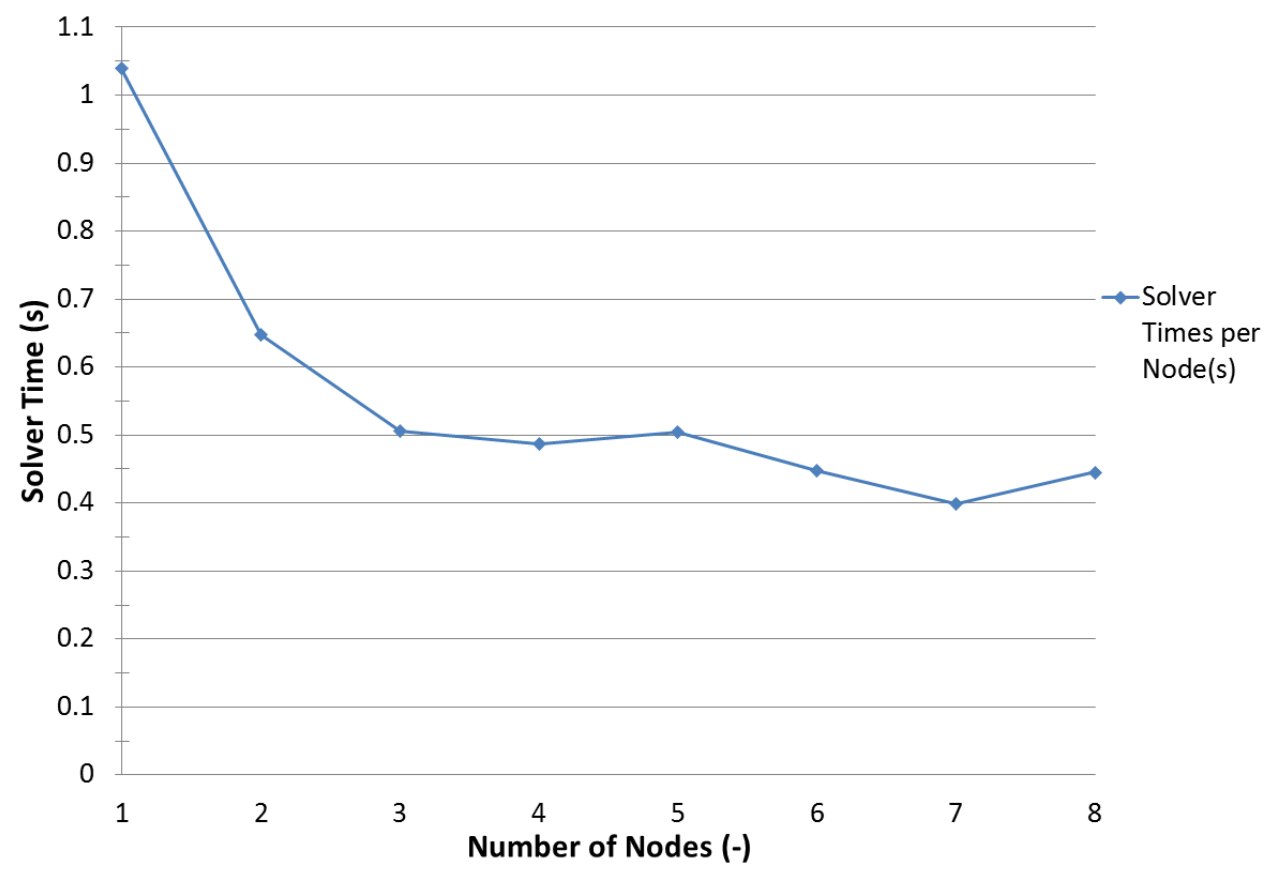

Figure 4.5: Solver time per time step as a function of the number of cluster compute nodes using 8 processor cores per node

A color contour plot of the depth of the scour hole after two hours of scour is shown in Figure 4.6. This model does not include sediment transport, and therefore there is not a pile of sediment in the downstream of the pier that would be expected if sediment transport with settling of the sediment were included in the model. The geometry and conditions for the model match those of one of the cylindrical pier scour experiments in [2], and the general bowl shape of the scour hole is very similar to that of the experiment. The flow conditions create a bed shear that is somewhat greater than critical over the entire bed for $0.25 \mathrm{~mm}$ sand and some general scour of the entire bed occurs and leaves a higher bed at the entry, exit, and flume wall at the end of the scour time period. 


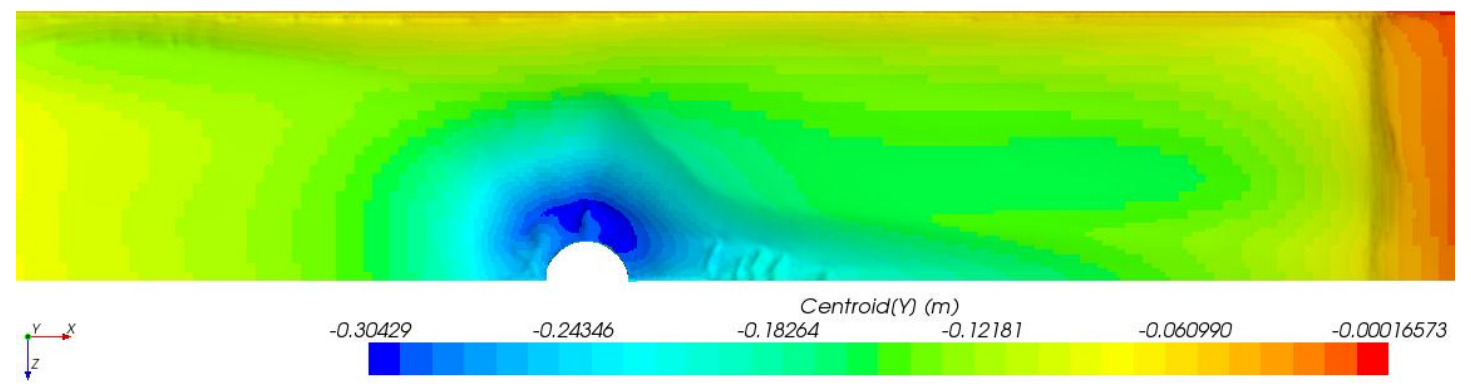

Figure 4.6: Sediment bed contours after 2 hours of scour with all 3 model enhancements showing bowl shaped scour hole

Bed contours at periodic time intervals from the edge of the pier at $z=-0.1$ to the flume wall at $z=-0.4$ at the $x=0$ position are shown in Figure 4.7. The slope of these contours is less than the angle of repose of the sand except near the pier wall, notably at $300 \mathrm{~s}$ and $900 \mathrm{~s}$. The sand slide model appears to have reduced the slopes near the pier wall by $1800 \mathrm{~s}$, except for a small remaining upward bend very close to the pier wall.

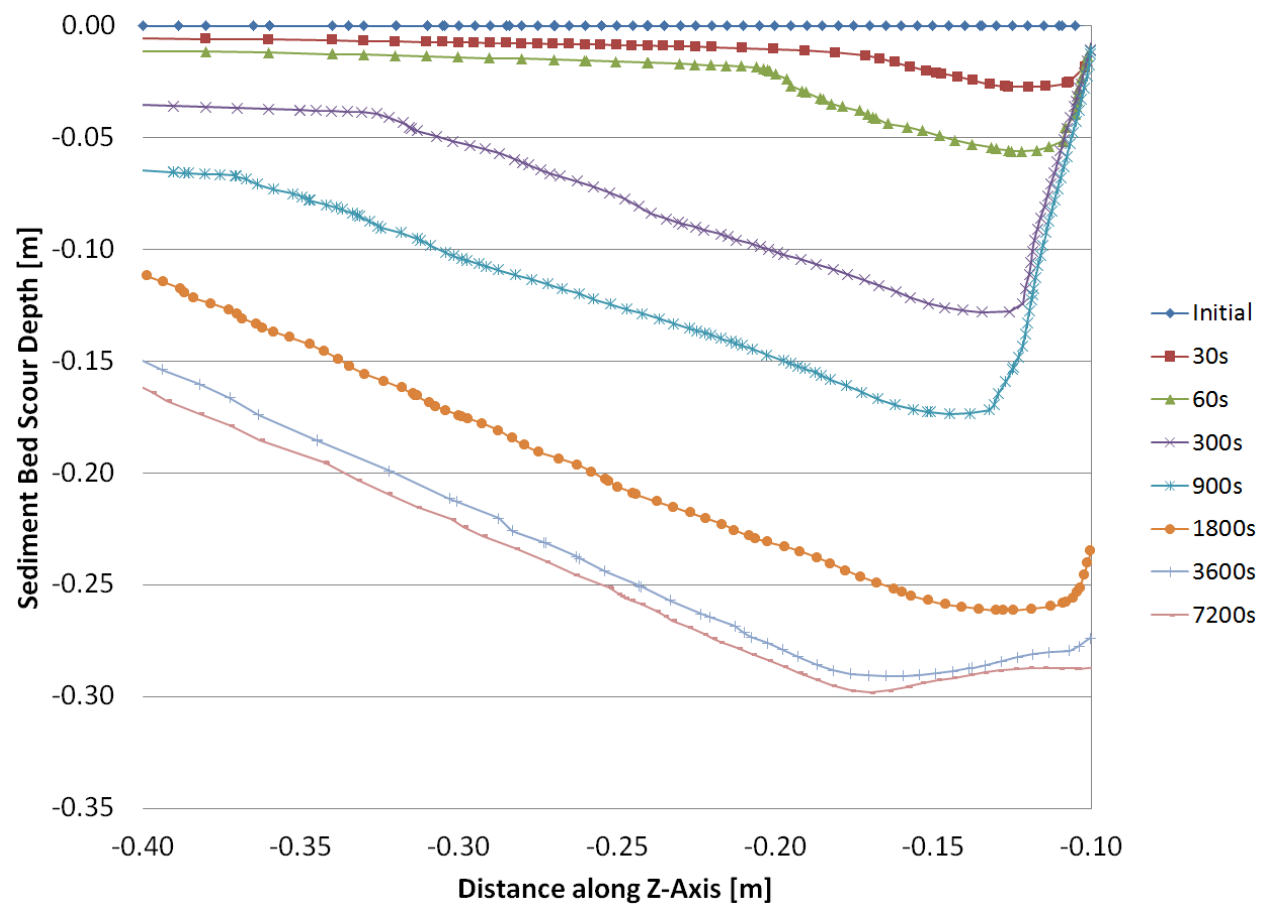

Figure 4.7: Evolution of scour hole profiles across the bed from $(x=0$ line $)$ from the edge of the pier at $z=-0.1$

After the two hour scour time, the bed shear stress is low, at or below the critical shear stress as shown in Figure 4.8 and Figure 4.9. Critical shear stress for $0.25 \mathrm{~mm}$ sand for the initial flat bed is $0.18 \mathrm{~Pa}$. The variation of the critical with the contour of the scoured bed is shown in Figure 4.9. Critical shear stress is lower than $0.18 \mathrm{~Pa}$ when the bed slop is downhill with respect to the shear stress vector direction on the surface and greater than the flat bed critical shear stress when the bed slope is uphill with respect to the shear stress vector on the surface. Because the flow velocity created conditions for general as well as pier scour there is an uphill zone near the end of the flume where the critical shear stress is higher than 
$0.18 \mathrm{~Pa}$. A similar higher critical shear stress area can be seen around the downstream side of the scour hole around the pier.

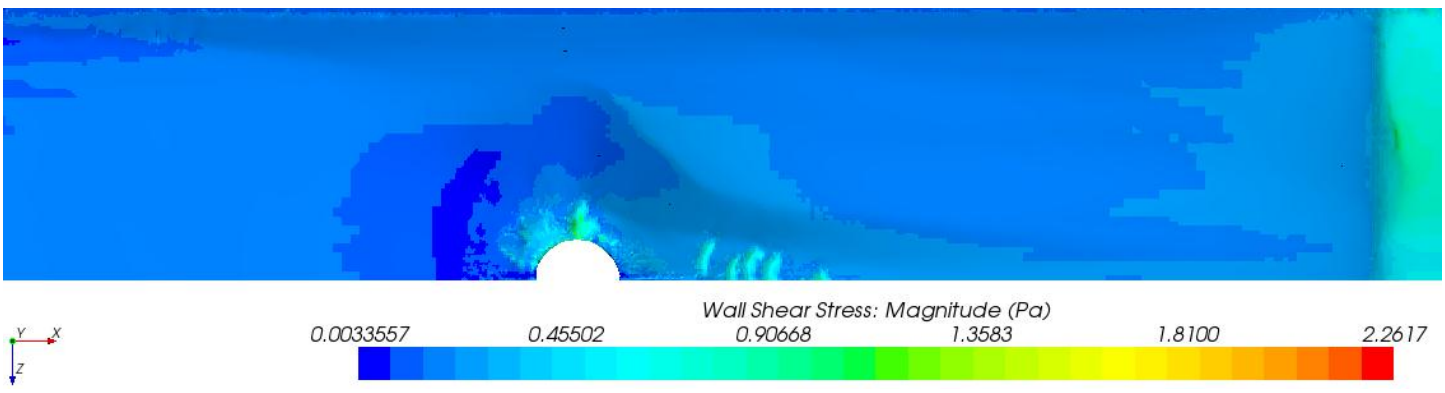

Figure 4.8: Sediment bed shear stress magnitude after 2 hours of scour

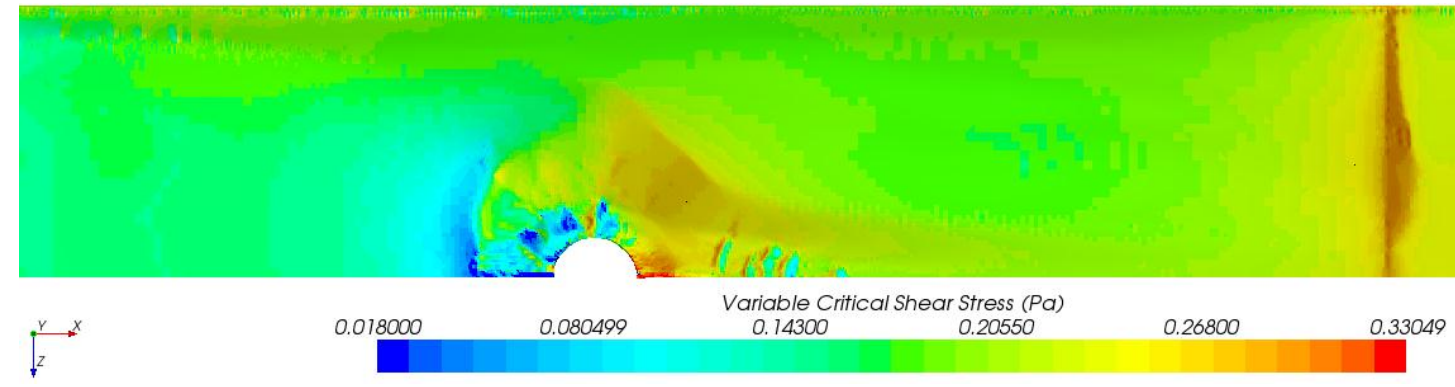

Figure 4.9: Sediment bed critical shear stress after 2 hours of scour showing low values on downslopes and higher values on up slopes with respect to horizontal in the direction of the shear stress vector

The angle of repose for sand in the sand slide model was taken to be 33 degrees. A color plot of the angle of the bed with respect to the horizontal is shown in Figure 4.10. The simple sand slide model can be seen to be fulfilling its primary function of keeping the bed angle less than or equal to the angle of repose. The thin red lines where the angle of the bed is greater than the angle of repose are the flume and pier walls that are excluded from sand slides.

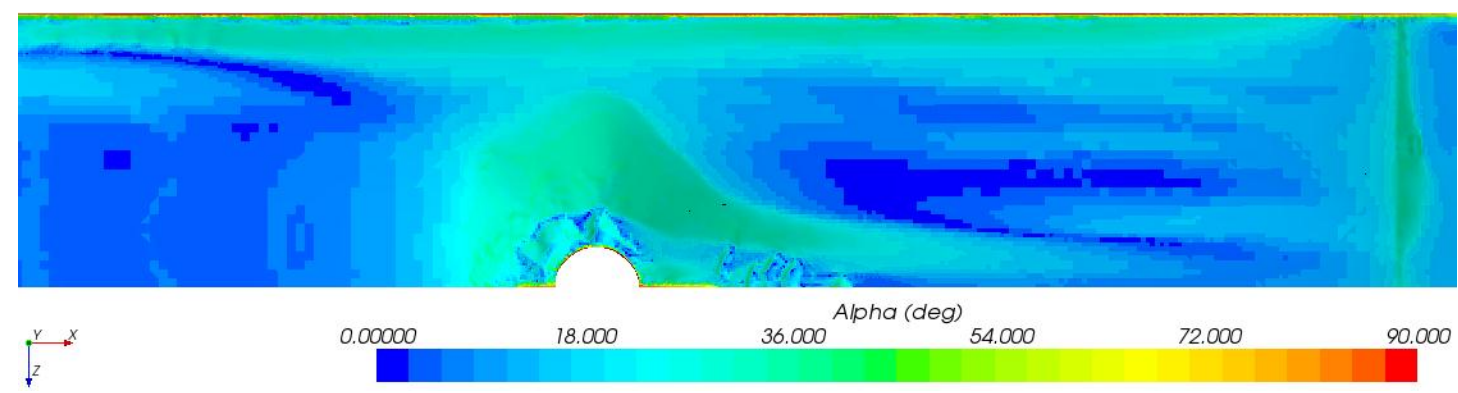

Figure 4.10: Bed slope angle after 2 hours of scour

Figure 4.11 shows the scoured bed contour under identical flow conditions but with successive addition of the variable critical shear stress model in (a), the addition of the model enhancement to make the scour displacements normal to the bed and employ variable critical shear stress in (b), and finally in (c) 
the application of all three model enhancements, the first two and the sand slide model. These results appear to show that the sand slide model is the most important among the three enhancements in obtaining a broad rounded bowl shape of the scour hole that corresponds fairly closely to that of the experimental result obtained in [2].

The next step in the work plan to reach a complete model will be to test sediment transport options. The Eulerian particle transport model that is an existing capability in STAR-CCM+ appears to be the best candidate to model sediment transport due to its scalability in parallel computing and ability to handle arbitrarily large numbers of particles. Some issues with the current state of the model yet remain and will continue to need attention. Mesh morphing eventually leads to large bed displacements in the scour application which require fairly frequent boundary extraction and remeshing of the domain to maintain stability of the computation. Some accuracy loss is incurred for a short time interval after the remeshing operation because the existing solution state must be interpolated to the new mesh and that operation is not conservative. The two hours of time to form the scour hole currently requires more than a week of wall time to compute, and therefore, some effort applied to making the model more efficient is also needed. 


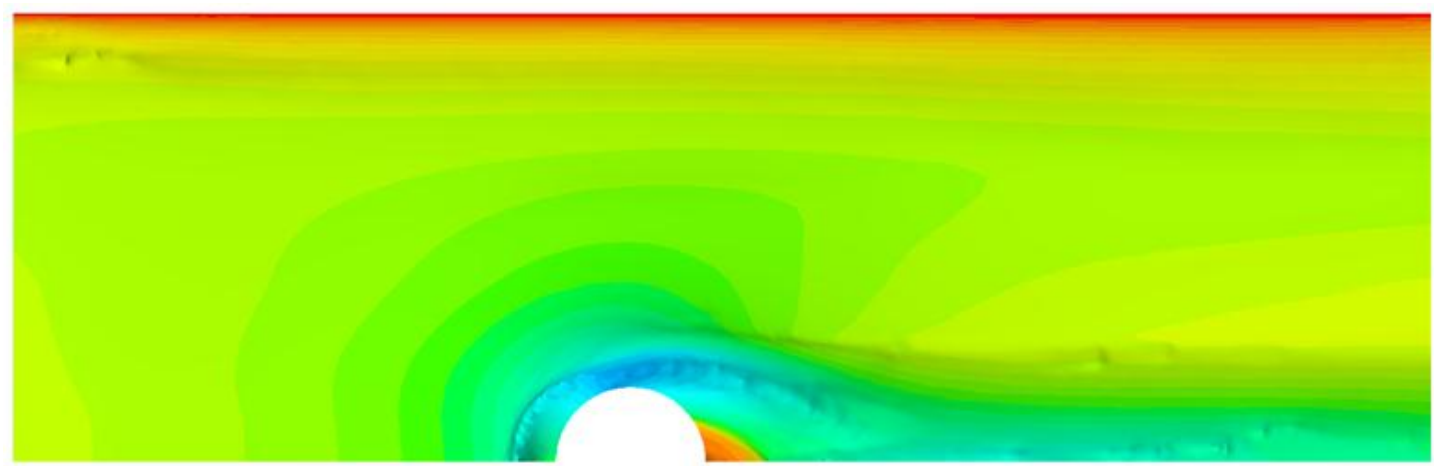

(a) VCSS Scour Hole

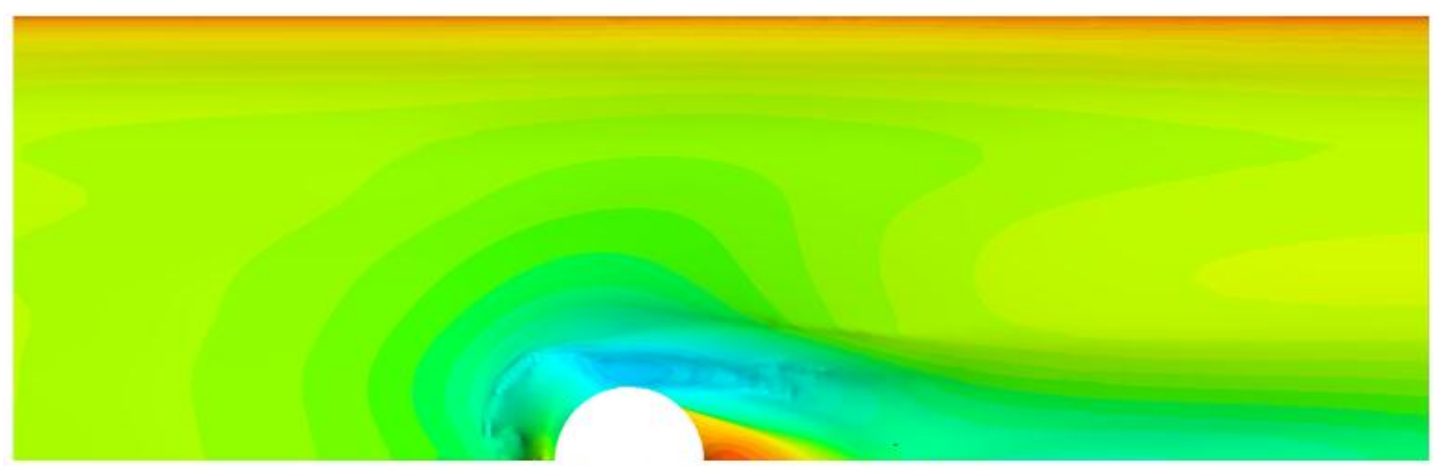

(b) Normal Scour Hole

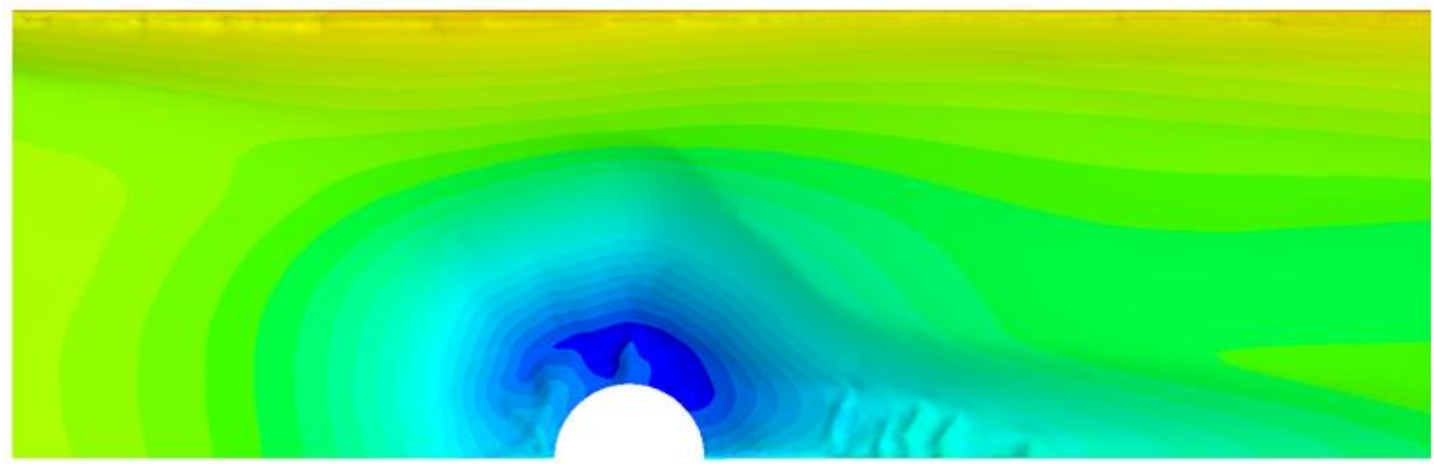

(c) Sandslide Scour Hole

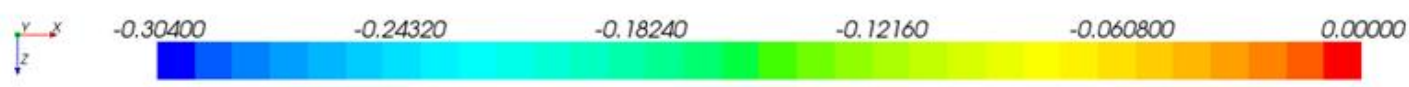

Figure 4.11: Comparison of scour hole contours after 2 hours of scour with successive model enhancements: (a) uses only variable critical shear stress, (b) adds scour displacement normal to the bed, and (c) adds the sand slide model

\subsubsection{References}

1. Leo van Rijn, "Sediment Pick-Up Functions," Journal of Hydraulic Engineering, Vol. 110, No. 10, October 1984, pp. 1494-1502.

2. Niels Bohrs, Offshore Wind Turbines situated in Areas with Strong Currents, Offshore Center Danmark, Doc. No. 6004RE01ER1, 2006. 
3. Chris Edwards, "3-D Mesh Morphing Iterative Methodology for Flow Scouring around Bridge Piers Implemented in a Commercial CFD Code," Master's Thesis, Northern Illinois University, 2011.

\subsection{Outreach, User Support, Training and Technology Enablement}

The videoconferences held every other week among collaborators working on the TFHRC project are continuing and have been both very useful and successful. There are frequently one or two topics that are of highest priority and these consume most of the time of the meeting. It is scheduled to run for two hours but often runs overtime. In addition to sharing current research results, alternative methods to resolve current issues are discussed and a plan of action or actions for the next two weeks are determined. These are followed up with email exchanges as needed. A bridge engineer from California DOT is working on using the open source CFD software OpenFOAM on the TRACC cluster and has joined the biweekly videoconferences. 


\section{Multi-Dimensional Data Visualization}

\subsection{Introduction}

During this quarter development continued on both the TransimsVIS and TransimsEDT applications. The TransimsVIS development was done as part of the RTSTEP project which is covered in full under the work for others section.

Development of TransimsEDT included the addition of transit-related features to the core network editing capabilities. Transit editing has traditionally been a large challenge for TRANSIMS users as any network changes made will tend to disrupt the transit network built on top of it. This is due to the fact that the transit routing is fairly unintelligent about how to correct small gaps in the route.

More work on including transit models into the visualization software is reported in the work for others section, as much of this work is currently being funded by the RTSTEP project. 


\section{Computational Structural Mechanics Applications}

Tasks for Computational Structural Mechanics Applications in year five include:

- Technical support for Computational Structural Mechanics

- Collaborator Research Reports

- Multi-physics Analysis of Bridge Components

- CSM Outreach, User Support, Training and Technology Enablement

\subsection{Technical Support for Computational Structural Mechanics}

LS-DYNA usage on the cluster varies but frequently all 500 licenses are in use (see Figure 6.1). The average usage over the last three months is equivalent to 213 cores with continuous load (compared to 190 in the first quarter and 244 in the second quarter of 2011). In the recent quarter, the heaviest users of the LS-DYNA package were Florida state University, NHTSA and TRACC with Northern Illinois University (NIU).

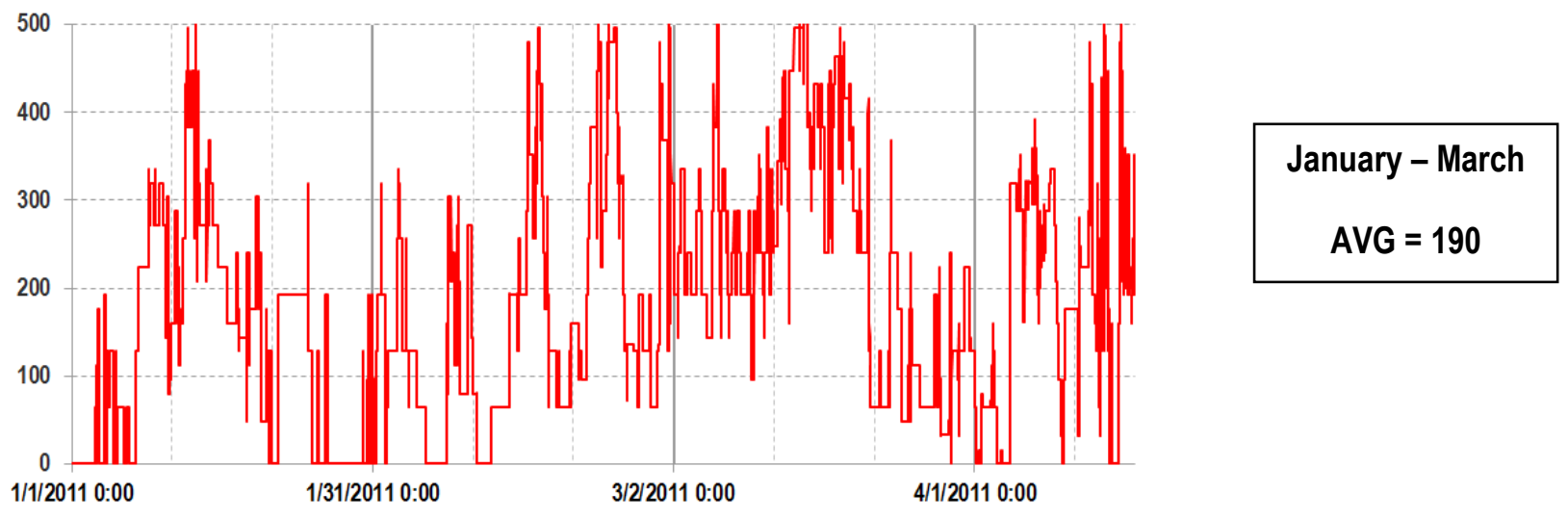


Figure 6.1a: LS-DYNA usage on the TRACC Cluster in the first quarter of 2011

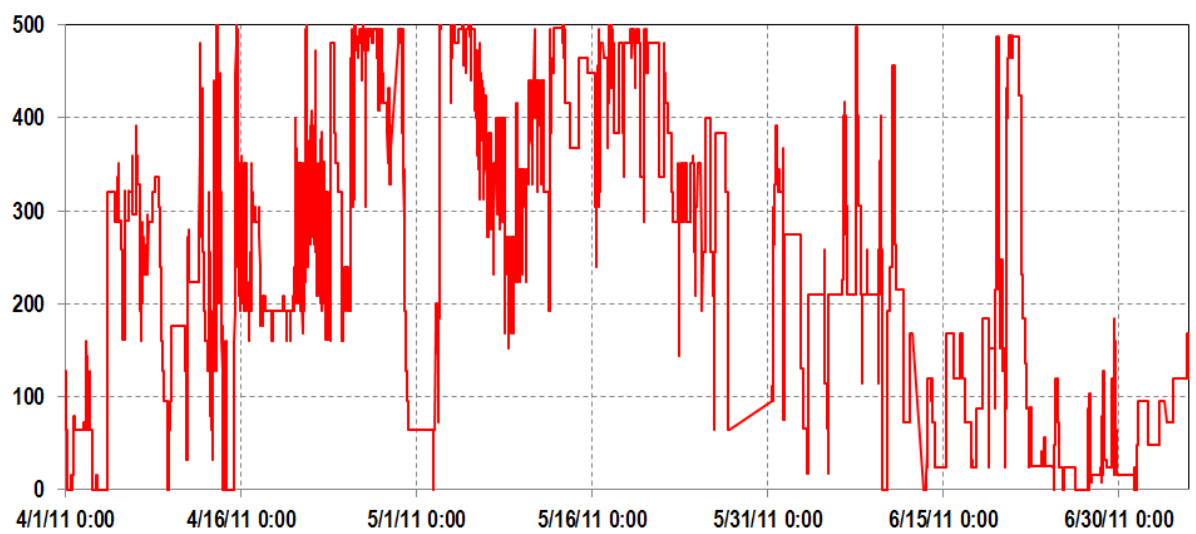

\section{April - June \\ AVG $=244$}

Figure 6.1b: LS-DYNA usage on the TRACC Cluster in the second quarter of 2011

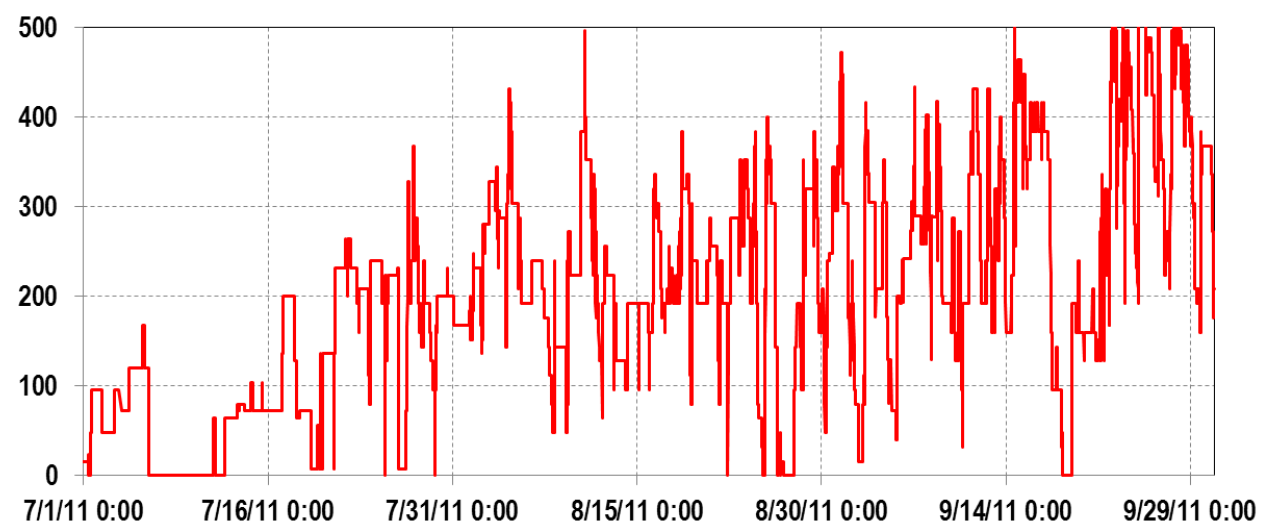

July - September
AVG $=\mathbf{2 1 3}$

Figure 6.1c: LS-DYNA usage on the TRACC Cluster in the third quarter of 2011

\subsection{Collaborator Research Reports}

\subsubsection{Florida Agriculture and Mechanical University - Florida State University College of Engineering}

The following is a description of the support given to FAMU-FSU and a terse presentation of their research results. FAMU-FSU College of Engineering is currently working on project: Best Practice Guidelines for Construction of Paratransit Buses (FDOT contract No. BDK83-943-10).

\subsubsection{Introduction}

In the recent quarter FAMU-FSU researchers were working on finalizing their year-long project and preparing the final reports. Significant number of simulations was performed in order to validate their FE models before they are delivered to the sponsors. Previously conducted full rollover experimental tests gave a lot of insight into the bus behavior during the impact. Two major discrepancies noticed 
during the experiment were friction coefficient of the concrete slab and fracture behavior of the windshield in the FE simulation of the $800 \mathrm{~mm}$ rollover test.

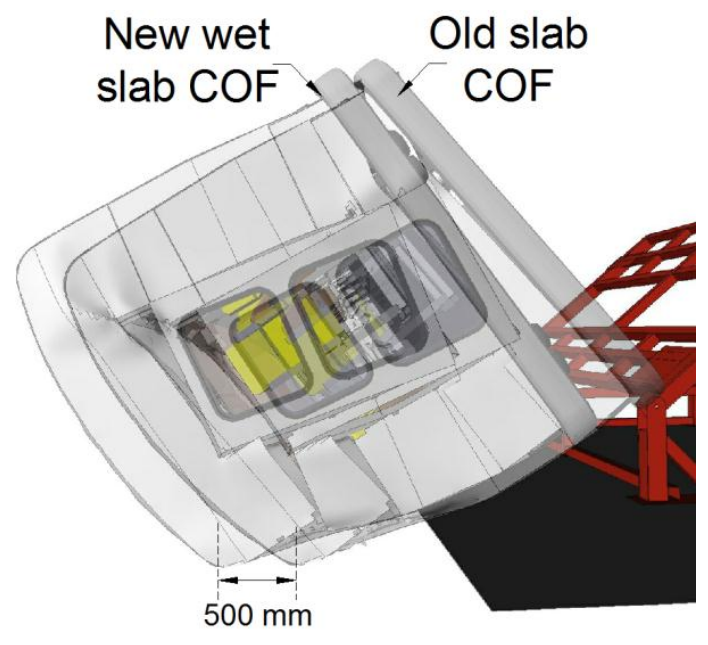

Figure 6.2: Comparison of FE simulations with and without adjustment of COF of concrete slab

\subsubsection{FE Model Updates in the Validation Process}

The new concrete slab constructed for the most recent rollover test (described in the previous quarterly report) is smoother than the previous one. Additionally, the slab became wet on the day of the test due to rainfall. These two factors largely influenced the coefficient of friction on the slab. It was noticeable during a full scale rollover test that the bus slid on the concrete pad right after the impact (see Figure 6.2). This effect was not anticipated for in the rollover simulation, where the coefficient of friction was large enough so that almost no sliding occurred. To obtain a better value for the simulation COF it was decided to use the Parameter Optimization feature in LS-OPT software.

The amount of sliding measured during the full scale rollover at the back wall was approximately $500 \mathrm{~mm}$ (see Figure 6.2) in the direction pointing away from the tilt table (negative y direction in the model). This data was used to calibrate the friction coefficient of the wet slab using the parameter optimization feature in LS-OPT software. Since the sliding displacement in the experiment was measured in the left top back corner of the bus, this point was selected as a reference. In the FE simulation a target function was specified as a displacement of a selected node in the y direction. It has been calculated that the total $y$ displacement of this node in the simulation was: $1193 \mathrm{~mm}$ (distance in the y direction from the position of unstable equilibrium of the bus on the tilt table until the moment of impact) $+500 \mathrm{~mm}$ (distance of the back wall sliding on the concrete pad), which resulted in the total displacement of 1693 $\mathrm{mm}$ as a target value. The results of all the iterations have been shown in Figure 6.3, where fparam is a coefficient of friction on the concrete slab. It was calculated that the desired coefficient of friction of the concrete slab was 0.231 as shown in Figure 6.4. 


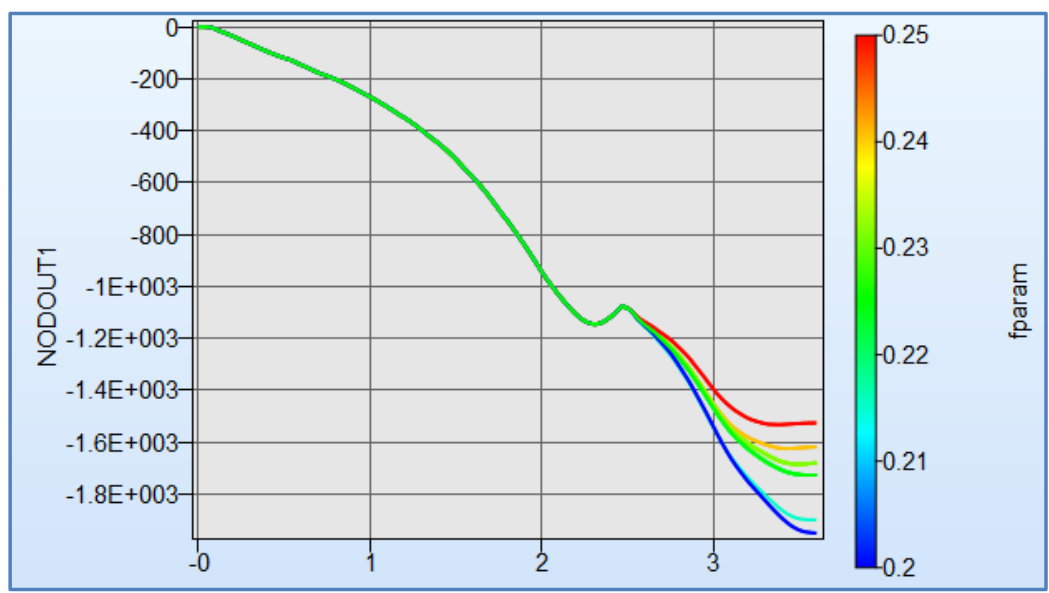

Figure 6.3: Coefficient of friction (COF) of wet concrete slab calibration results. Time vs. displacement in the y direction of the selected node, multiple iterations have been shown

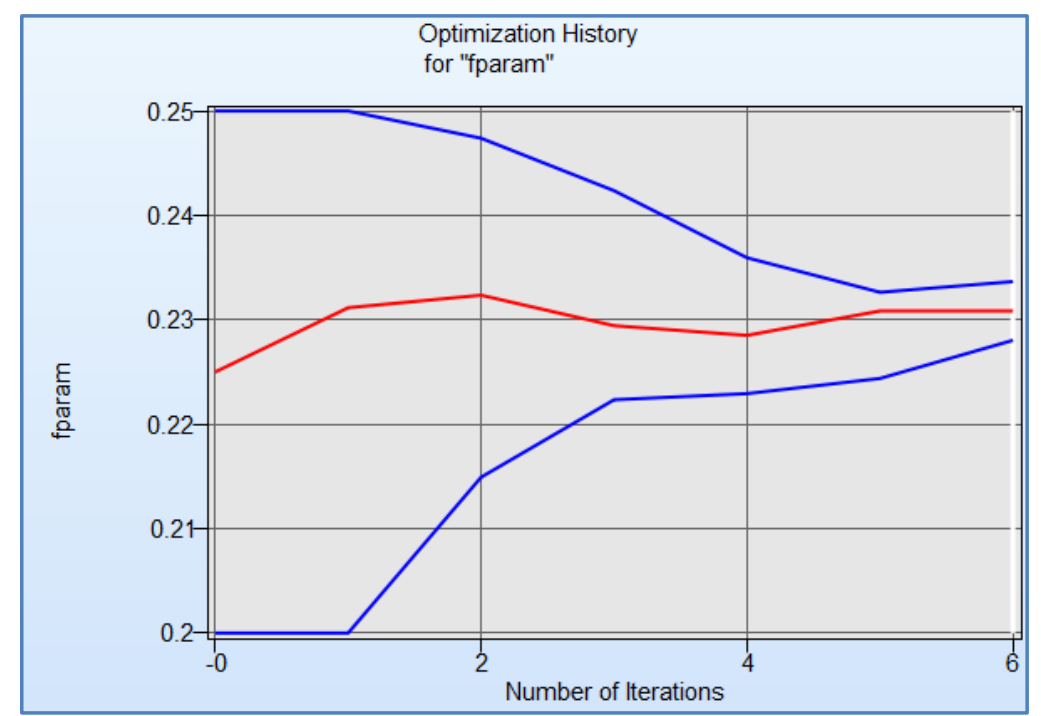

Figure 6.4: Optimization history for coefficient of friction (fparam) of wet concrete slab

Another important discrepancy noticed during a full scale rollover test was the performance of the windshield FE model. Safety glass, a material used for windshields, is a very difficult material to simulate. The structure of safety glass, a layer of PVB composite sandwiched in between two pieces of glass, adds to this complexity. Although the material properties for glass itself are pretty well established, the PVB is a more complex material with a high strain rate dependency. The material model implemented in the existing FE model was based on research done by Du Bois, Kolling, and Fassnacht [1] with a MooneyRivlin formulation for PVB. As defined in the Ls-Dyna manual, the Mooney-Rivlin material formulation is based on the strain energy density function defined as: 


$$
W=A(I-3)+B(I I-3)+C\left(I I I^{-2}-1\right)+D(I I I-1)^{2}
$$

Where:

$$
\begin{gathered}
C=0.5 A+B \\
D=\frac{A(5 v-2)+B(11 v-5)}{2(1-2 v)}
\end{gathered}
$$

$v$ - Poisson's ratio,

$2(A+B)-$ shear modulus of elasticity,

$I, I I, I I I$ - Invariants of right Cauchy-Green Tensor.

This leads to the expression for engineering stress (Piola-Kirchhoff stress) given by:

$$
\boldsymbol{S}=2(A+B I) \mathbf{1}-2 B \boldsymbol{C}+4\left(D J^{2}\left(J^{2}-1\right)-C J^{-4}\right) \boldsymbol{C}^{-1}
$$

Where:

$$
\begin{aligned}
& \boldsymbol{S} \text { - Piola-Kirchhoff stress tensor, } \\
& \mathbf{1} \text { - Identity matrix, } \\
& \boldsymbol{C} \text { - Right Cauchy-Green strain tensor, } \\
& J \text { - Determinant of the deformation gradient. }
\end{aligned}
$$

The values defining the Mooney-Rivlin material formulation proposed by Du Bois, Kolling, and Fassnacht, were: $A=1.60 \mathrm{MPa}$, and $\mathrm{B}=0.06 \mathrm{MPa}$. Unfortunately these results were based on quasi-static experiments for PVB, which made them difficult to use in the experiments with a higher strain rate. This resulted in the windshield completely failing in the FE model whereas it did not in the full scale rollover (drop height of $800 \mathrm{~mm}$ ). This was not the case in the previous rollover test (drop height of $909 \mathrm{~mm}$ ), where both FE model and full scale bus windshields failed, and it was impossible to identify this discrepancy.

The review of existing papers on PVB properties [2] allowed identification of material properties for higher strain rates and their implementation into the Mooney-Rivlin material model. The obtained values were: $\mathrm{A}=1.00 \mathrm{MPa}$, and $\mathrm{B}=9.00 \mathrm{MPa}$. Comparison of both formulations is shown in Figure 6.5. 


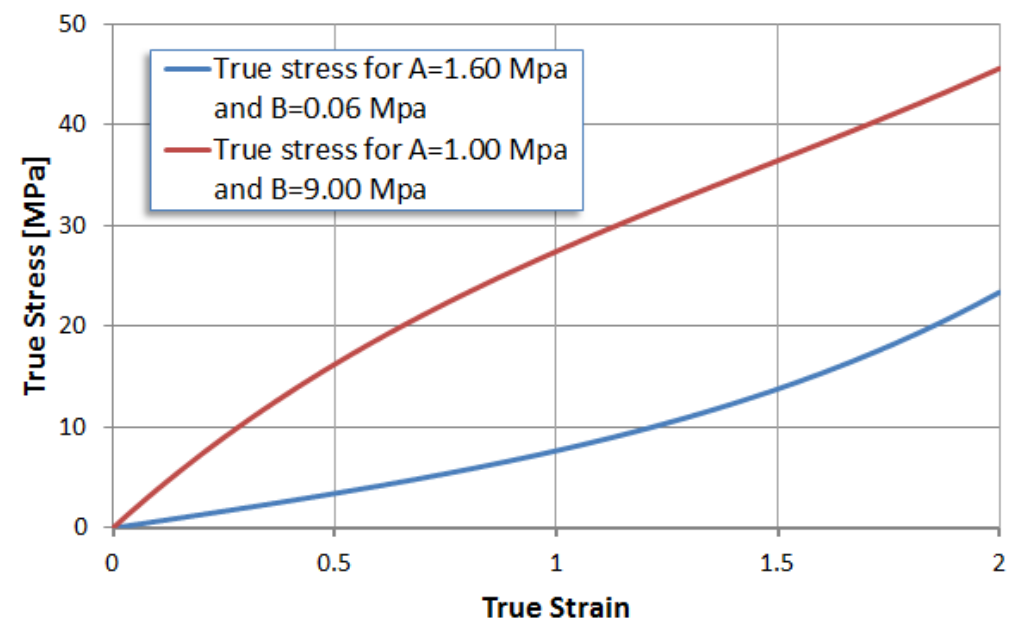

Figure 6.5: Comparison of PVB formulation for different parameters

After implementation of these changes the FE simulations were repeated. The before and after results are shown in Figure 6.6, it may be noticed that the windshield with the newly defined material parameters for PVB does not break up.
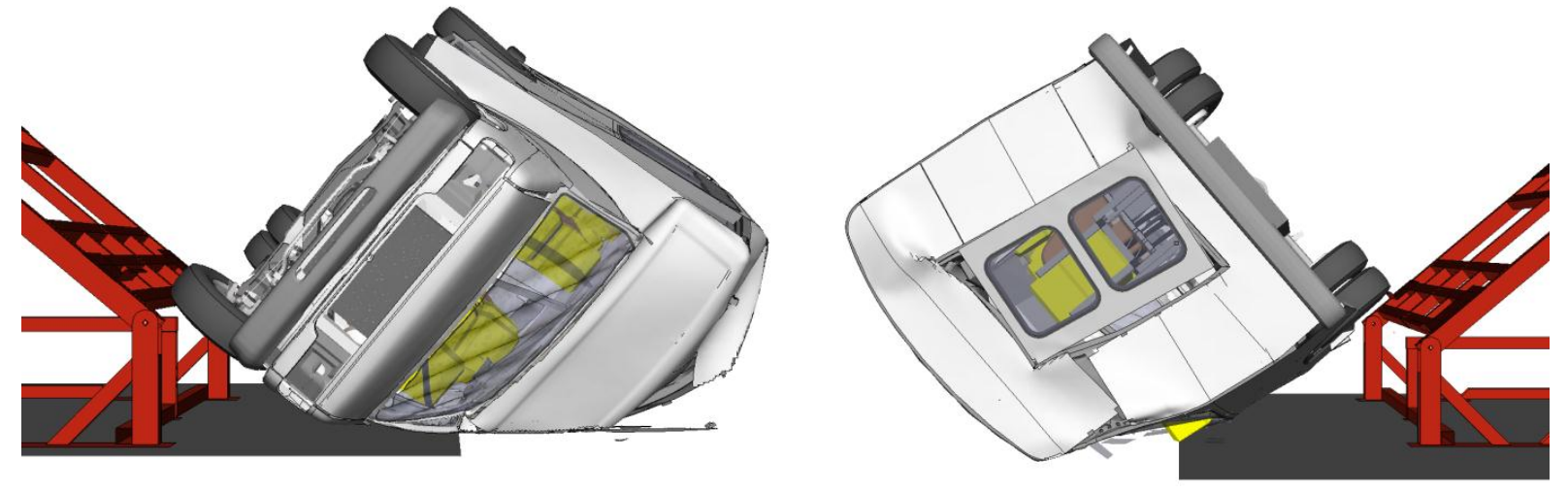

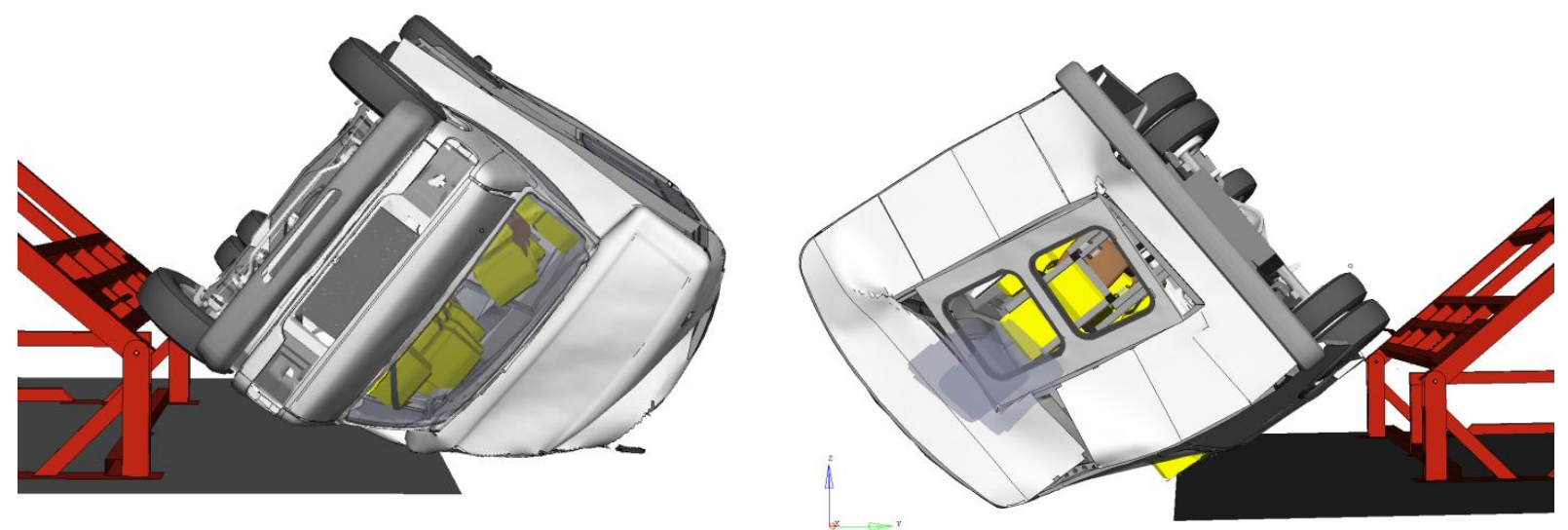

Figure 6.6: A comparison of results from the initial FE simulation of $800 \mathrm{~mm}$ rollover test (top) and after calibration (bottom)

The location of the string transducers used in the experiment for measurement of the deformations is shown in Figure 6.7. The figure also shows residual space and comparison of the wall deformations. The simulations are in good agreement with the experiment.

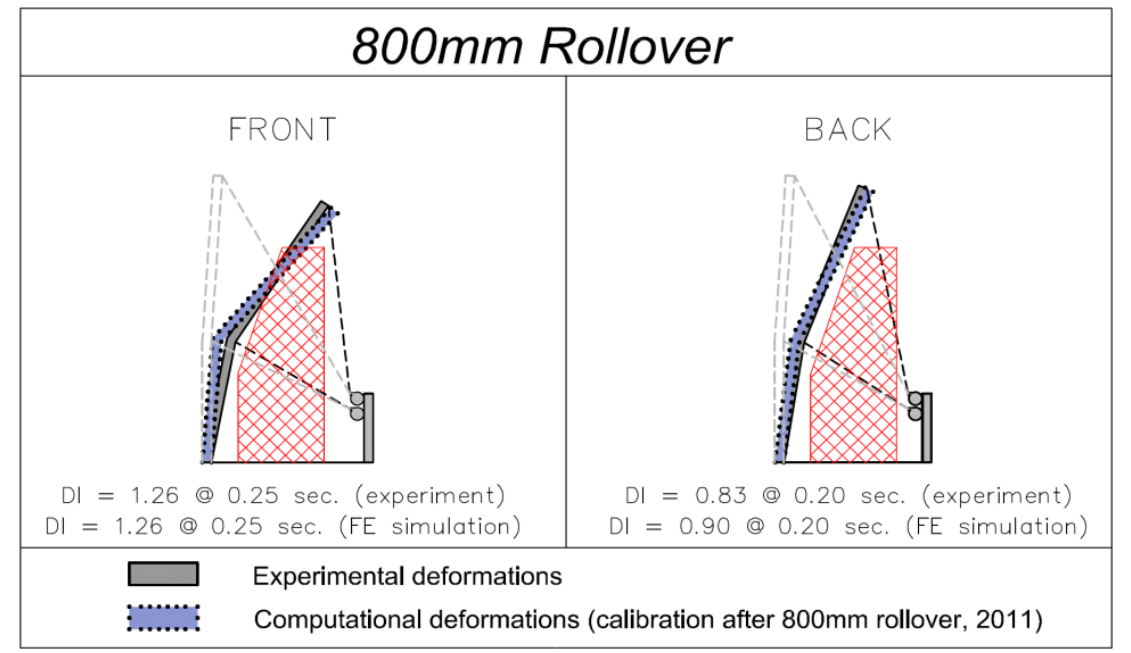

Figure 6.7: Comparison of experimental and numerical results from the $800 \mathrm{~mm}$ rollover

The string transducer displacement results are compared with experimental data in Figure 6.8 and Figure 6.9. 


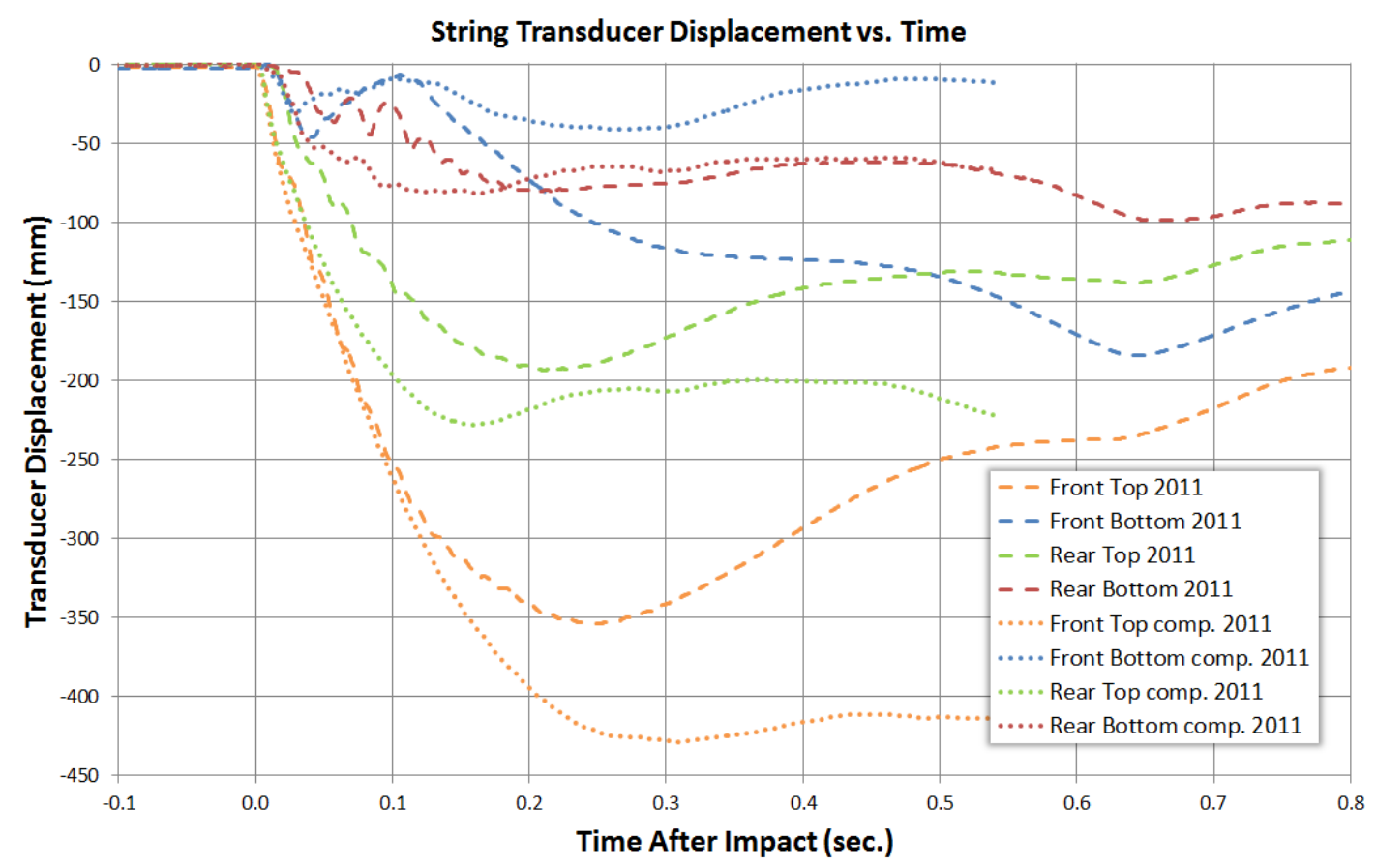

Figure 6.8: Comparison of string transducer displacement and numerical results from an $800 \mathrm{~mm}$ rollover

After the calibration process was completed the FE model simulation corresponding to the 2010, $909 \mathrm{~mm}$ rollover test was rerun. The string transducer displacement results are compared with previous model and the experiment in Figure 6.9. The new FE model with implemented changes showed an improved agreement with the experimental data. 


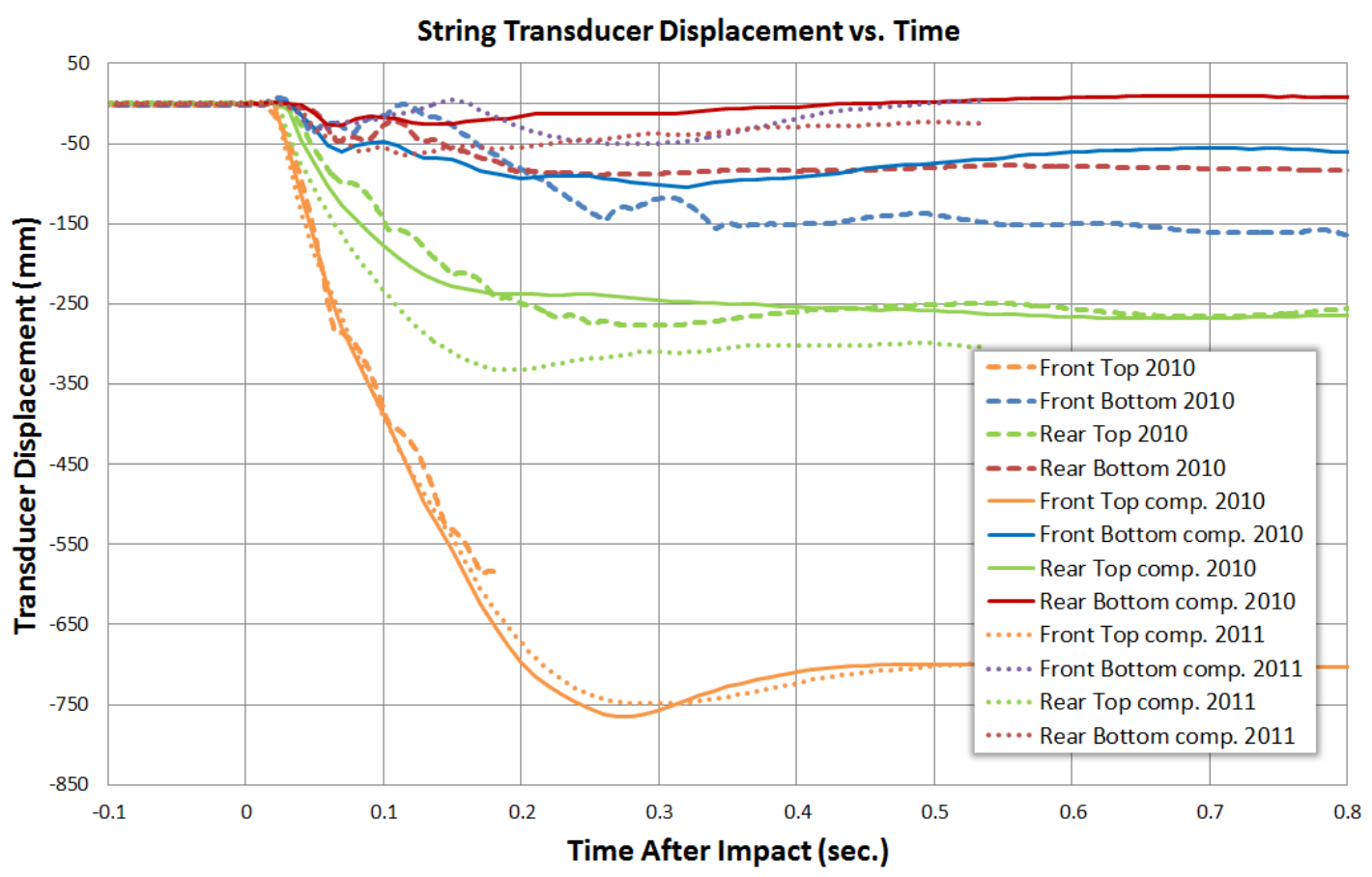

Figure 6.9: Comparison of string transducer displacement and numerical results from a 909mm rollover

\subsubsection{Improvement of the Bus Design}

Analysis of the Paratransit bus rollovers conducted on the Springhill FDOT testing facliity has identified that for paratransit buses the connection between the chassis cab and front portion of the body structure is a very important factor in determining how the bus performs in the rollover. The reason for such behavior is a structure of paratransit buses. First, a cage rear wall, works as a very stiff shear wall during the rollover, and limits the cage deformation in the back of the bus. Second, the Center of Gravity (COG) of the paratransit bus is located near the front of the bus, which delivers the resultant inertial force during the rollover to the front of the bus. Both of these result in large deformations of the frontal section of the bus.

The purpose of this study was to investigate possible ways of improving this critical area of bus to body connection. The approach was to use the calibrated FE model of a paratransit bus, developed during previous research and apply a number of design changes that could improve crashworthiness behavior during the rollover test. The selection of proposed design changes was based on the simplicity of their implementation in a real bus. All of the following changes are only meant to be representative of possible changes. Actual implementation of these would be the responsibility of the manufacturer. All of the developed FE models were then subjected to the Florida Standard computational rollover test.

Design changes were applied in a step by step manner, one design change per simulation. Once it was discovered that a given design modification improves the rollover crashworthiness (lowers the Deformation Index, DI), the next one was implemented for a new iteration step. The applied changes 
that gave the best results are shown in the figures below. Figure 6.10 shows additional columns and plates connecting chassis cab with the bus cage implemented into the FE model.
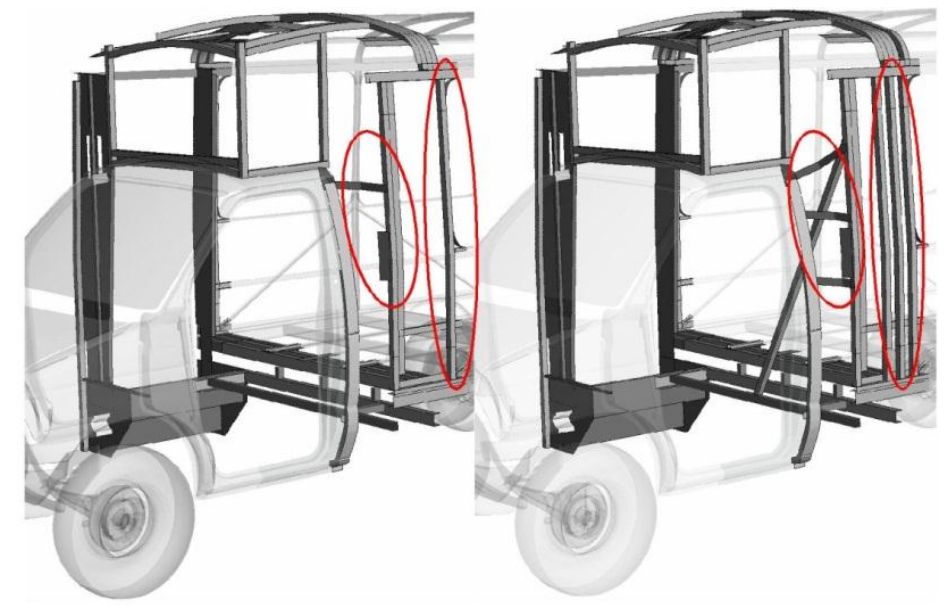

Figure 6.10: Additional columns and plates connecting cab chassis and bus cage implemented into the model - original design on left, improved design on right

It was discovered that in both FE simulation and full scale tests, one of the major structural elements failing during the rollover was a steel doorframe section. During the rollover experiment the steel frame would rupture at the connection with entrance steps (see Figure 6.11), resulting in a deformation of the entire curbside sidewall. Such behavior was due to the width change in the steel plate element, causing the curb effect to occur. This problem was solved by eliminating the reduction in the frame width and adding additional steel plate to the design that was welded to a side wall.

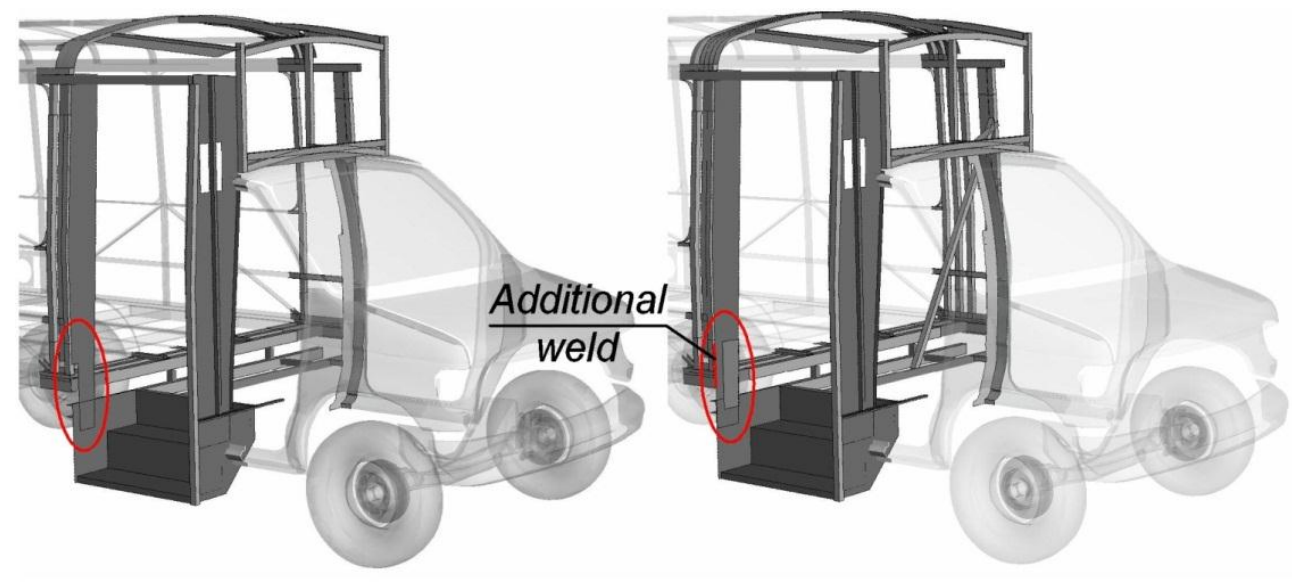

Figure 6.11: Design change of the steel doorframe - original design on left, improved design on right

To protect the roof structure from bowing, during the rollover test, an additional roofbow was added to the cage structure and the thickness of the three bows was increased from 16ga to 14ga. 
To brace the street side wall structure a diagonal cross member was implemented into the design, (see Figure 6.10).

Table 6.1 shows the Deformation Index (DI), for all the performed iterations. (Note: the Deformation Index is less than 1 for acceptable designs. When it is greater than 1 , the structure penetrates the residual space and the bus fails the test).

Also a change in mass of the vehicle was shown to underscore the weight cost of each implemented design change.

Table 6.1: Deformation Index and total mass of a loaded vehicle for each design (iteration)

\begin{tabular}{|c|c|c|}
\hline Design \# & Max Deformation Index & Mass of a Loaded Vehicle (tonne) \\
\hline 0 & 1.262 & 5.922 \\
\hline 1 & 1.252 & 5.922 \\
\hline 2 & 1.240 & 5.925 \\
\hline 3 & 1.178 & 5.928 \\
\hline 4 & 1.230 & 5.929 \\
\hline 5 & 1.188 & 5.933 \\
\hline 6 & 1.172 & 5.933 \\
\hline 7 & 1.133 & 5.934 \\
\hline 8 & 1.175 & 5.935 \\
\hline 9 & 1.167 & 5.941 \\
\hline 10 & 1.170 & 5.934 \\
\hline 11 & 1.162 & 5.936 \\
\hline 12 & 1.008 & 5.939 \\
\hline
\end{tabular}

The final deformation of a bus structure before and after the cab connection design improvement is shown in Figure 6.12. This study shows how important the frontal section of the bus structure for the crashworthy rollover behavior. A well design frontal ring is a critical component for a bus intended to pass a Florida Standard rollover test. 

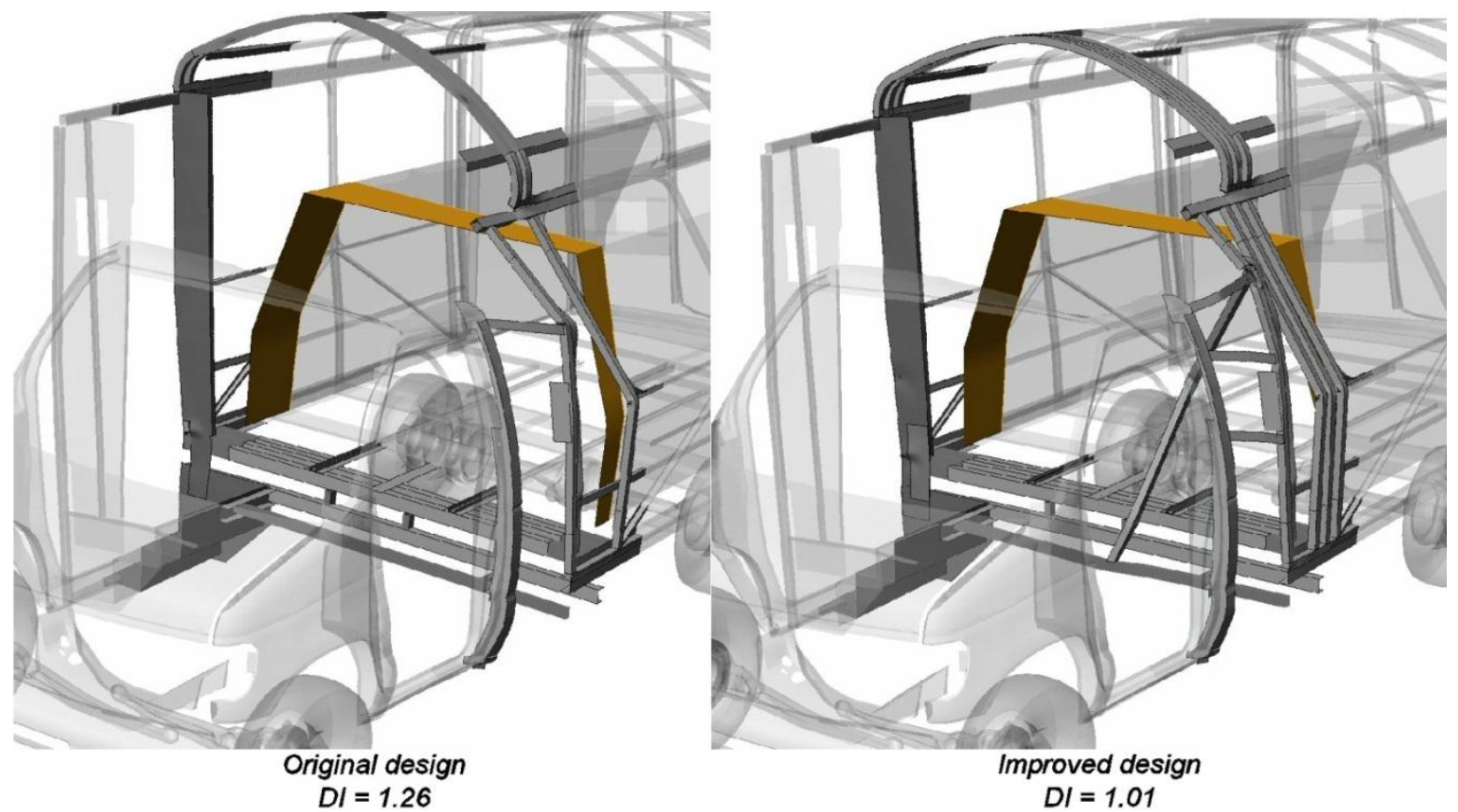

Figure 6.12: Final deformation of a bus structure - original design on left, improved design on right

\subsubsection{References}

1. Du Bois P.A., Kolling S., Fassnacht W., Modeling of safety glass for crash simulation. International Journal of Computational Material Science, 2003, vol. 28(3-4), pp. 675-83.

2. Iwasaki, R., Sato, C. , Latailladeand, J. L. and Viot, P., Experimental study on the interface fracture toughness of PVB (polyvinyl butyral)/glass at high strain rates, International Journal of Crashworthiness, 2007, vol. 12(3), pp. 293-298. 


\subsection{Bridge Response to Blast Loading}

\subsubsection{Introduction}

In the previous quarters of this project, TRACC focused mainly on studying the literature and exploring LS-DYNA capabilities in regard to modeling blast loading effects. Also benchmark tests for further LSDYNA simulations were chosen. For the purpose of validation, a benchmark experiment reported previously by Pope and Tyas [1] was studied. This case focused primarily on the techniques of modeling the blast load in LS-DYNA and not much on the material behavior, since the target steel plate was treated as rigid.

The recent studies performed at TRACC on the effect of blast loading on the concrete deck of the Bill Emerson Memorial Bridge have shown the importance of appropriate modeling of concrete failure. The simulation of concrete failure in LS-DYNA is frequently modeled by a simple erosion algorithm that fails elements when a given stress or strain component (or their invariants) exceeds predefined limit value. The limit values are not easy to determine and are frequently assumed ad hoc without any experimental support. Thus, engineering methods of determining the failure of the concrete should be investigated in more detail.

\subsubsection{Simulation of Blast loading on a Square Reinforced Concrete Plate}

In this quarter response of a reinforced concrete square plate to blast loading was studied. As a benchmark validation experiment conducted and reported by A.G. Razaqpur [2] was chosen. In the test eight doubly reinforced concrete plates with the dimensions as in Figure 6.13 were clamped and subjected to blast load. The $1000 \times 1000 \times 70 \mathrm{~mm}$ square plates were made from concrete with an average compressive strength of $42 \mathrm{MPa}$. The charge was placed at the stand-off distance of $3.0 \mathrm{~m}$ above the plate's center. Several sets of experiments were performed for the plates with and without GFRP reinforcement. In the current analysis only the RC plates without GFRP external reinforcement were studied. The steel bar yield and ultimate strength were measured to be $480 \mathrm{MPa}$ and $600 \mathrm{MPa}$ respectively. For the analyzed case the charge of $33.4 \mathrm{~kg}$ of ANFO was used in the experiment. ANFO was composed of $5.7 \%$ of fuel oil and $94.3 \%$ of ammonium nitrate and shaped to a nearly spherical form. The explosive energy of ANFO is $3717 \mathrm{~kJ} / \mathrm{kg}$ which is about 0.82 of the energy of one kilogram of TNT. Thus, the load was equivalent to load exerted by $27.34 \mathrm{~kg}$ of TNT.

In the tests blast wave characteristics (i.e. incident and reflected pressures and impulses), central deflection of the panel and mode of failure were registered. 


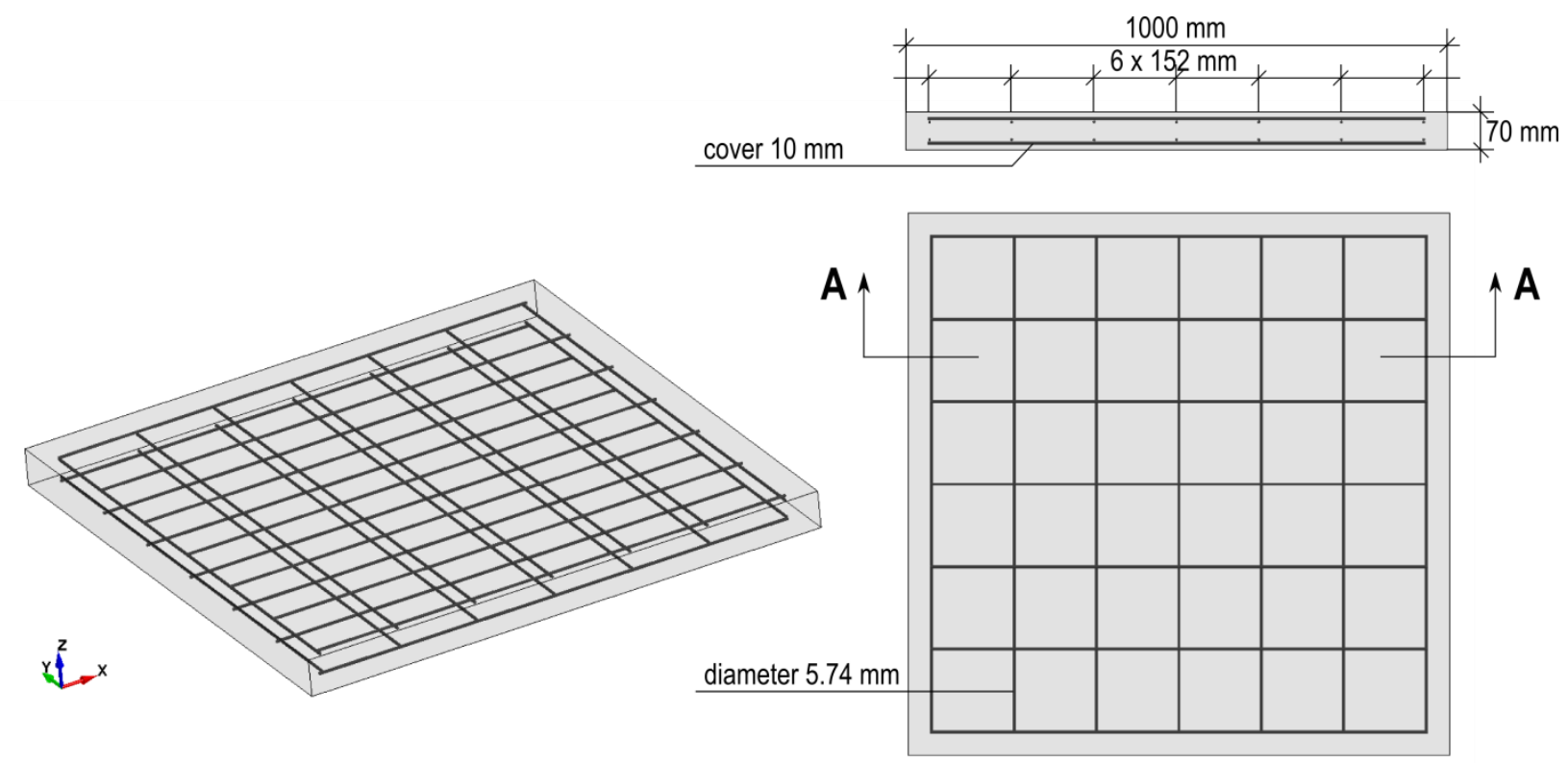

Figure 6.13: Test specimen geometry and reinforcement details

\subsubsection{Finite Element Model}

The LS-DYNA model of the concrete plate was built using 70,000 single integration point solid elements with a cubical shape and edge dimensions of $10 \mathrm{~mm}$ (7 layers through the thickness of the slab). The reinforcement steel was modeled using 2,576 Hughes-Liu beam elements embedded in the concrete by using the CONSTRAINED_LAGRANGE_IN_SOLID command. The concrete was simulated by three different material models: MAT_072R3 concrete with damage material model, MAT_084 Winfrith concrete and MAT_159 continuous surface cap model. All these material models allow the user to input material properties or generate a set of properties based on a limited number of inputs. The generated data are based on built in empirical equations created from experimental data on regular concrete. All of the material models allowed for incorporation of strain rate effects in the concrete.

Table 6.2: Simplified LS-DYNA input for MAT_072R3 (units system metric ton, $\mathrm{mm}, \mathrm{sec}$ )

\begin{tabular}{|l|l|l|l|l|l|l|l|}
\hline ID & RO & PR & & & & & \\
\hline 1 & $2.277 \mathrm{e}-009$ & 0.16 & & & & & \\
\hline FT & a0 & a1 & a2 & & & & \\
\hline & -42.00 & & & & & & \\
\hline slambda & nout & edrop & rsize & ucf & Icrate & locwidth & npts \\
\hline & 2 & & 0.03937 & 145 & 2 & & \\
\hline
\end{tabular}

Where non-zero values:

RO - density of material 
PR - Poisson's ratio

a0 - if negative value, unconfined compressive strength for parameter generation

RSIZE - unit conversion factor for length inch/user-unit

UCF - unit conversion factor for stress psi/user-unit

LCRATE - load curve for strain rate effects

Table 6.3: Simplified LS-DYNA input for MAT_084 (units system metric ton, $\mathrm{mm}$, sec)

\begin{tabular}{|l|l|l|l|l|l|l|l|}
\hline ID & RO & TM & PR & UCS & UTS & FE & ASIZE \\
\hline 1 & $2.277 e-009$ & $3.068 \mathrm{e}+004$ & 0.16 & 42.00 & 3.77 & 0.08654 & 19.00 \\
\hline E & YS & EH & UELONG & RATE & CONM & CONL & CONT \\
\hline & & & & & -4.0 & & \\
\hline
\end{tabular}

Where non-zero values:

TM - Tangent modulus

UCS - Uniaxial compressive strength

UTS - Uniaxial tensile strength

FE - Fracture energy

ASIZE - Aggregate size

CONM - Unit system metric ton, $\mathrm{mm}$, sec

Table 6.4: Simplified LS-DYNA input for MAT_159 (units system metric ton, mm, sec)

\begin{tabular}{|l|l|l|l|l|l|l|l|}
\hline ID & RO & NPLOT & INCRE & IRATE & ERODE & RECOV & ITRETRC \\
\hline 3 & $2.277 \mathrm{e}-009$ & 1 & 0.0 & 0 & 0.0 & 0 & 0 \\
\hline PRED & & & & & & & \\
\hline 0.0 & & & & & & & \\
\hline FPC & DAGG & UNITS & & & & & \\
\hline 42.00 & 19.00 & 2 & & & & & \\
\hline
\end{tabular}

Where non-zero values:

FPC - Unconfined compression strength

DAGG - Maximum aggregate size

UNITS - Unit system metric ton, $\mathrm{mm}$, sec

TRACC/USDOT Y5Q4 
The steel was simulated using MAT_024 piecewise linear plasticity model. Strain rate effects were simulated for both the steel as well as the concrete materials. Since minor concrete erosion was expected in this study, the LOAD_BLAST_ENHANCED command was used to simulate the blast wave. In the case when extensive failure of material is expected, the blast wave should be modeled explicitly by Multi Material Arbitrary Lagrangian Eulerian approach.

\subsubsection{Comparison of Simulation Results with Experimental Tests}

Table 6.5 compares results between the two experimental tests and the LS-DYNA simulation. The reflected pressure, arrival time for the wave and maximum plate deflection are very well predicted. The reflected impulse is underestimated by the numerical model.

Table 6.5: Test and simulation results comparison

\begin{tabular}{|c|c|c|c|c|}
\hline Case & $\begin{array}{c}\text { Reflected } \\
\text { Pressure (kPa) }\end{array}$ & $\begin{array}{c}\text { Reflected Impulse } \\
\text { (kPa ms) }\end{array}$ & $\begin{array}{c}\text { Arrival Time } \\
\text { (ms) }\end{array}$ & $\begin{array}{c}\text { Plate Max. } \\
\text { Deflection (mm) }\end{array}$ \\
\hline Experiment 1 & 5528 & 1954 & - & 13.12 \\
\hline Experiment 2 & 5712 & 2412 & 1.50 & 9.53 \\
\hline Simulation MAT_072R3 & 5384 & 1757 & 1.52 & 9.90 \\
\hline Simulation MAT_084 & 5384 & 1757 & 1.52 & 12.32 \\
\hline Simulation MAT_159 & 5384 & 1757 & 1.52 & 13.22 \\
\hline
\end{tabular}

In general, damage in LS-DYNA is a function of plastic strain. Many material models take into account the damage, but usually the definition of damage varies in different constitutive materials. Figure 6.14 shows the damage of the concrete for three tested material models. MAT_072R3 allows for displaying so called Damage Parameter that reaches value of 2.0 when the material is not able to take any more load. The damage parameter is not equivalent to the failure of the concrete material and cannot be interpreted as such. Explicit cracks in the concrete can be identified when MAT_084 is used. Different definition of damage is also used in the MAT_159.

The cracks in the experiment occurred on the tension side under the reinforcement. Figure 6.15 shows approximate locations of the biggest cracks on the plate surfaces in the experiments. For detailed pictures of the plates see [2]. 


\section{Compression side}
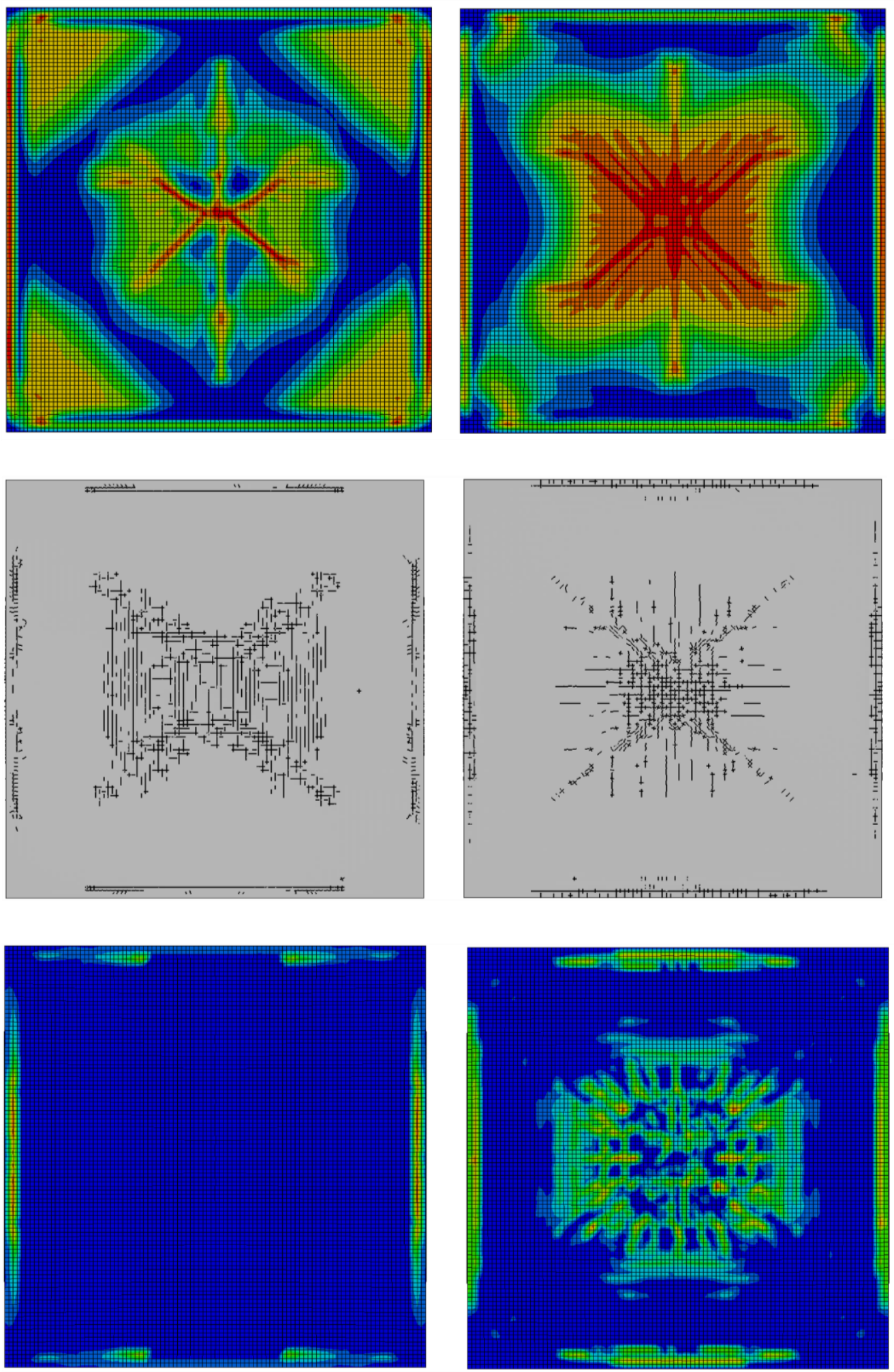

MAT_072R3

Damage

Parameter

$1.996 \mathrm{e}+00$

$1.796 \mathrm{e}+00$

$1.596 \mathrm{e}+00$

$1.397 \mathrm{e}+00$

$1.197 \mathrm{e}+00$

$9.978 \mathrm{e}-01$

7.982e-01

$5.987 \mathrm{e}-01$

3.991e-01

$1.996 \mathrm{e}-01$

$0.000 \mathrm{e}+00$

Figure 6.14: Damage in the plate after the blast event for different LS-DYNA material models 


\section{Experiment 1}
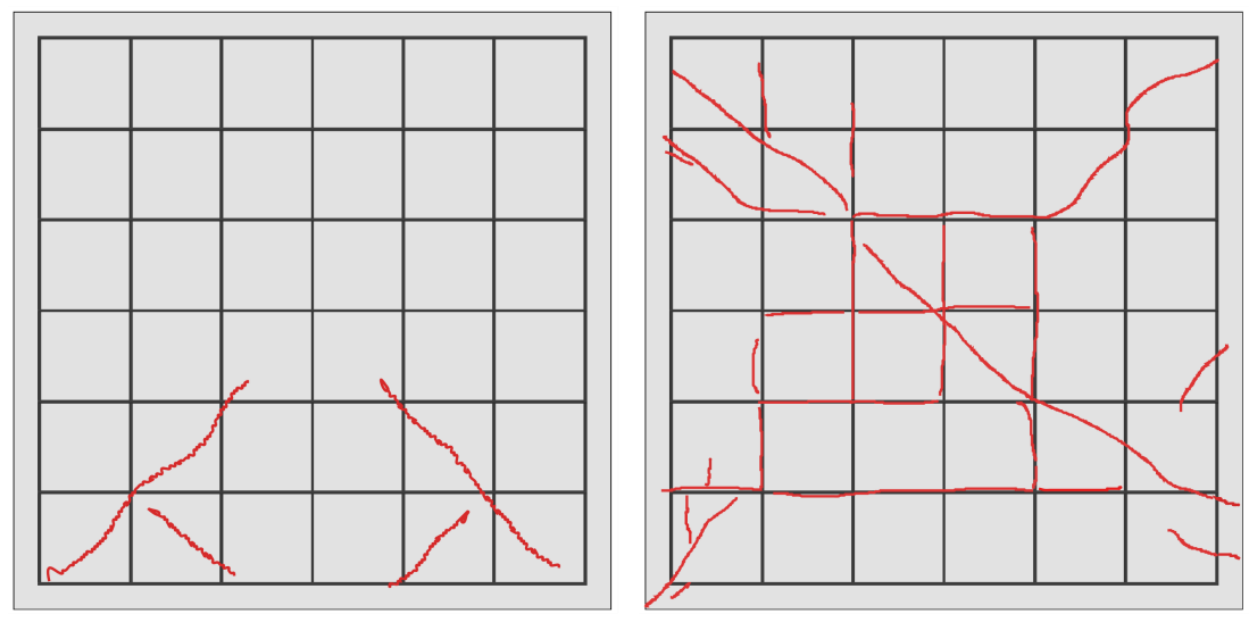

Experiment 2
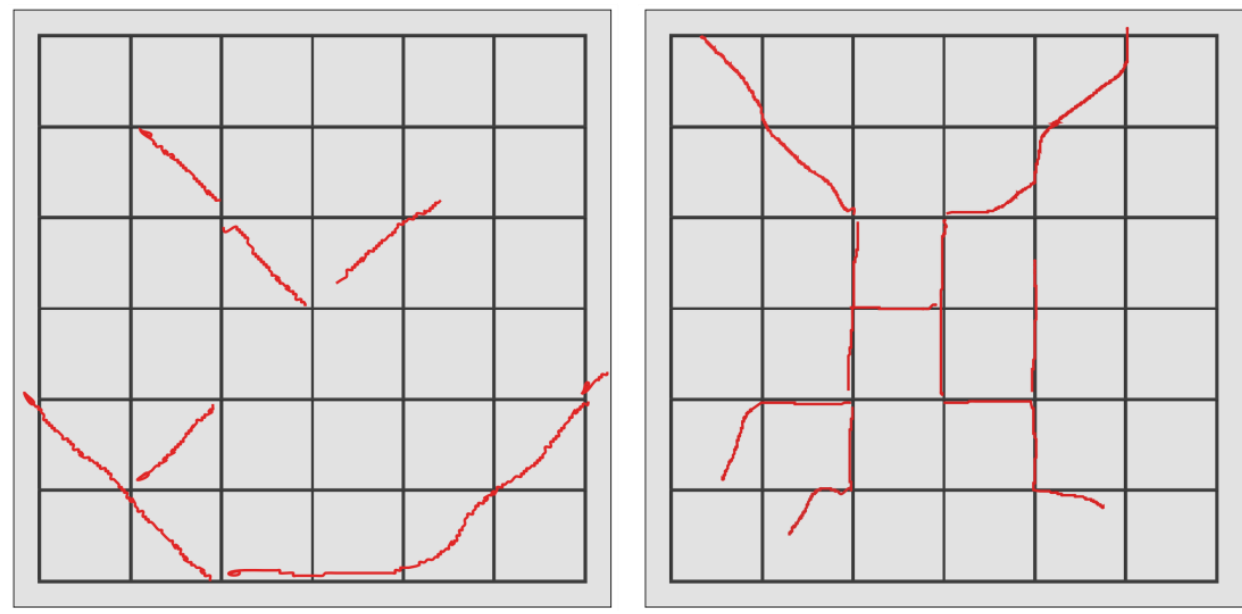

Figure 6.15: Approximate location of the cracks in the experiments

\subsubsection{Conclusions}

From the comparison of simulation results with experimental results, it can be concluded that all the models captured the behavior of the plate pretty well. The MAT_072R3 gave the stiffest response - the deflection of the plate was considerably less than the deflection predicted by the two other models. Winfrith concrete material model allows for displaying the predicted location of cracks; this is very useful for comparison to the experiments. The locations of the cracks - especially on the tension side (bottom) of the plate - were accurately predicted. 


\subsubsection{References}

1. Pope, D. J., Tyas, A., Use of hydrocode modelling techniques to predict loading parameters from free air hemispherical explosive charges. 1st Asia-Pacific Conference on Protection of Structures Against Hazards, Singapore, 2002.

2. Razaqpur, A. G., Tolba, A., . Contestabile, E., Blast loading response of reinforced concrete panels reinforced with externally bonded GFRP laminates, International Journal of Composites, Part B 38 (2007) 535-546.

\subsection{CSM Outreach, User Support, Training and Technology10.1080/13588265.2011.616118 Enablement}

\subsubsection{Outreach}

TRACC continuously develops and maintains its own Wikipedia site. The page contains descriptions of the current software and documents the usage of that software on the TRACC Cluster. It has become an invaluable source of information to the current cluster users and also to a broader community of computational mechanics researchers.-I don't have any updates.

CSM staff hosted the following visitors and provided and overview of the capabilities and research being performed by CSM:

- Dr. Tyler Ley, Assistant Professor of Civil and Environmental Engineering, and Dr. Jay Hanan, Assistant Professor of Mechanical \& Aerospace Engineering, Oklahoma State University (August 5, 2011)

- >Rick Kreifeldt, Vice President, Global Automotive Research and Innovation Corporate Technology Group, HARMAN Automotive/HARMAN International and Robert Boatright, Director of Research for HARMAN International's CTO Office (August 8, 2011).

- Professors and students, University of Illinois at Chicago's Computational Transportation Sciences IGERT Tour (August 17, 2011).

- $\quad$ Sixteen attendees, Patrick Engineering (September 21, 2011).

TRACC has been performing research in the area of crashworthiness simulations of paratransit buses. One paper describing TRACC's research in this area was published by the International Journal of Crashworthiness: Cezary Bojanowski and Ronald F Kulak, Multi-objective optimization and sensitivity analysis of a paratransit bus structure for rollover and side impact test, International Journal of Crashworthiness, vol. 16, issue 6, 2011, pp. 665-676, DOI:10.1080/13588265.2011.616118

\subsubsection{User Support}

The current CSM users have been using the cluster and software for some time and have become seasoned users requiring minimal support. LSTC, the developer of the LS-DYNA suite of codes, did not have any new releases during this quarter. TRACC staff keeps all the versions of the software on the cluster and leaves it up to the user to select a version. No specific version is recommended for different 
types of the analysis. All the available versions of LS-DYNA are implemented in the qsub-dyna-gui tool, which was developed by the CSM staff. The tool turned out to be very useful for submitting and tracking LS-DYNA jobs

\subsubsection{Training}

A central point for computational structural mechanics training provides all the US organizations doing transportation related research with awareness and accessibility to the latest approaches, methods, software training and best practices that can be applied to the wide variety of transportation research needed to ensure a viable transportation infrastructure. The CSM group has a suite of training videos that are available http://www.anl.gov/TRACC/Training/CSM/index.html.

During this quarter, the CSM staff was preparing the next course offering, which will be entitled Parallel Computing and LS-DYNA on the TRACC Cluster. Currently, the plans are to offer the course in the late winter or early spring time frame. Following our recent protocol, both onsite and internet attendance will be available, and the course will be recorded for future viewing over the web. 


\section{Work for Others - RTSTEP - Regional Transportation Simulation Tool for Evacuation Planning}

This project has been performed at TRACC for the City of Chicago with funding from the Department of Homeland Security under the Regional Catastrophic preparedness Grant Program. The project started in December 2010 and lasted through August $30^{\text {th }}, 2011$. Some final work will be performed before November $30^{\text {th }} 2011$ to fine-tune the capabilities and provide appropriate training for emergency responders in the Chicago region.

The information in this chapter has been taken from a draft report on this project and highlights some of the aspects most interesting to other cluster users and sponsors at USDOT. The complete report is about 550 pages long and can be obtained upon request.

\subsection{Introduction}

Large-scale evacuations from major cities during no-notice events - such as chemical or radiological attacks, hazardous material spills, or earthquakes - have an obvious impact on large regions rather than on just the directly affected area. The scope of impact includes the accommodation of emergency evacuation traffic throughout a very large area; the planning of resources to respond appropriately to the needs of the affected population; the placement of medical supplies and decontamination equipment; and the assessment and determination of primary escape routes, as well as routes for incoming emergency responders. Compared to events with advance notice, such as evacuations based on hurricanes approaching an affected area, the response to no-notice events relies exclusively on preplanning and general regional emergency preparedness. Another unique issue is the lack of a full and 
immediate understanding of the underlying threats to the population, making it even more essential to gain extensive knowledge of the available resources, the chain of command, and established procedures. Given the size of the area affected, an advanced understanding of the regional transportation systems is essential to help with the planning for such events. The objectives of the work described here (carried out by Argonne National Laboratory) is the development of a multi-modal regional transportation model that allows for the analysis of different evacuation scenarios and emergency response strategies to build a wealth of knowledge that can be used to develop appropriate regional emergency response plans.

The focus of this work is on the effects of no-notice evacuations on the regional transportation network, as well as the response of the transportation network to the sudden and unusual demand. The effects are dynamic in nature, with scenarios changing potentially from minute to minute. The response to a radiological or chemical hazard will be based on the time-delayed dispersion of such materials over a large area, with responders trying to mitigate the immediate danger to the population in a variety of ways that may change over time (e.g., in-place evacuation, staged evacuations, and declarations of growing evacuation zones over time). In addition, available resources will be marshaled in unusual ways, such as the repurposing of transit vehicles to support mass evacuations. Thus, any simulation strategy will need to be able to address highly dynamic effects and will need to be able to handle any mode of ground transportation. Depending on the urgency and timeline of the event, emergency responders may also direct evacuees to leave largely on foot, keeping roadways as clear as possible for emergency responders, logistics, mass transport, and law enforcement.

This RTSTEP project developed a regional emergency evacuation modeling tool for the Chicago Metropolitan Area that emergency responders can use to pre-plan evacuation strategies and compare different response strategies on the basis of a rather realistic model of the underlying complex transportation system. This approach is a significant improvement over existing response strategies that are largely based on experience gained from small-scale events, anecdotal evidence, and extrapolation to the scale of the assumed emergency. The new tool will thus add to the toolbox available to emergency response planners to help them design appropriate generalized procedures and strategies that lead to an improved outcome when used during an actual event.

\subsection{Executive Summary}

The City of Chicago awarded the RTSTEP project to Argonne in mid-December 2010. Work on the project started right away to accomplish the rather large task in the short time frame allotted to the project. Several subcontractors had been lined up in advance, and appropriate contracts had been prenegotiated. These subcontracts could only be established after the acceptance of the master contract with the City, but Argonne was able to do so quickly because the RTSTEP project had been planned for a long time and the subcontracts were largely ready for signatures when the master contract was finally awarded.

The following subcontractors participated in the RTSTEP project: 
- $\quad$ AECOM - a top engineering and design firm. TRACC's contract was established with David Roden, a Senior Consulting Manager specializing in the development of advanced transportation simulation software. David Roden is the primary developer of USDOT'S TRANSIMS transportation systems modeling application the forms the basis for RTSTEP. AECOM developed the underlying simulation software components with special regards to the needs of the RTSTEP project. AECOM also brought their team with special expertise in the application of transportation models to the table, and worked both on the normal day model and the evacuation scenarios.

- IIT - The Illinois Institute of Technology has been working on TRANSIMS simulations in the City of Chicago for a couple years now, based on funding by the Federal Highway Administration. Their independent project also involved CMAP and CDOT, the Chicago Department of Transportation. IIT became a subcontractor in the RTSTEP project to provide hands-on labor on the detailed editing of the Chicago highway network, traffic signals, intersection details, and more, and also worked on the normal day model by establishing equilibration methodologies and test cases.

- NIU - Northern Illinois University has worked with TRACC in the past and has relevant experience in the application of TRANSIMS. NIU's existing work on pedestrian simulations and movement of individuals in buildings and special venues such as stadiums was used for the RTSTEP project to address the demand on the transportation system at special events.

- CMAP - The Chicago Metropolitan Agency for Planning was another important subcontractor and provided model components and data to the project. The RTSTEP project depends heavily on socio-economic data and the demand on and response of the underlying transportation network. CMAP originally provided their metropolitan simulation network to TRACC's TRANSIMS model, and has improved the network in a collaborative effort with TRACC and NIU several years back. As the coordinator of data collection, standardization, and distribution to the region, CMAP was an essential contributor to RTSTEP.

- $\quad$ NORC is part of the University of Chicago, and specializes in social science research and public policy. NORC supported TRACC's work on the evacuation decision survey that was part of the effort to determine appropriate demand on the transportation network. NORC reviewed the survey and helped shape it so that it is statistically relevant.

- Independent Contractors: A number of independent contractors working with Argonne have been involved with the project as well, such as in software development, system administration, consulting, meeting support, and general project management.

Starting in January 2011, bi-weekly coordination meetings were held with all subcontractors, OEMC, and others. These meetings were held at 10:00 on every second and fourth Thursday of the month. To document the RTSTEP effort, monthly reports were delivered to OEMC, detailing the ongoing work of all subcontractors and staff at Argonne. These meetings were particularly important to coordinate remote collaborators. With about 8 people at TRACC directly involved in the RTSTEP project, and 20 or more personnel working for the subcontractors, the team was sizable and effective communication was important to coordinate everybody's efforts. 
Training is an essential part, both in the development and application of RTSTEP. TRACC held a three day training course on TRANSIMS and the plans for RTSTEP on January 19 to 21, 2011. This training course was also broadcast over the internet to subcontractors and other people interested in this technology. Part of the training was repeated on February $24^{\text {th }}$ at OEMC due to the fact that some participants were unable to attend the training classes at Argonne due to their job responsibilities. Final training classes are scheduled for December 2011 and will be held at Argonne and at OEMC respectively.

A summary of work performed on each individual task is provided in the next chapter of this document. The project was mostly implemented by August 31, 2011, and OEMC and Argonne decided to implement a no cost time extension to wrap up some of the technical details and refine some of the features, such as the visualization capabilities, case studies, ortho-imagery, and such. OEMC and IEMA also established connections with GIS departments across the region to explore additional data sources useful for distribution with the final application. Work was finalize by November 30, 2011, and training courses will be held in December 2012 due to scheduling conflicts of participating individuals that made it advisable to postpone these training course until after the end of the project.

\subsection{Summary by Task}

\subsubsection{Task 1: Refinement and Extension of the Existing Road and Transit Network}

The original highway network of the TRANSIMS model was developed with a focus on analyzing the Chicago Business District in great detail. This effort was funded in 2008 by the Illinois Department of Transportation. Refined regional analysis requires refining other areas in the region to increase the fidelity of and applicability to emergency evacuations. The network improvements were implemented by TRACC staff and subcontractors from AECOM and IIT.

\subsubsection{Key Deliverables}

- Evaluation of data sources, methodologies, and available resources to upgrade the TRANSIMS highway network to support the regional aspects of emergency evacuation planning sufficiently. Results and recommendations will be discussed with OEMC in advance of actual network modifications.

- Implementation of a more robust TRANSIMS normal day model. The goal is to allow more flexibility when assigning different traffic patterns that are driven by a specific event and a specific emergency response to the event.

- Implementation of the network changes agreed upon between transportation modelers and emergency responders on the basis of a priority list. The implementation will be performed in several stages to allow stable intermediate networks to be available for the implementation of the other tasks under this statement of work.

- Documentation of all network changes, documentation of data sources and procedures, and electronic maps and hard copies of the improvements; integration with OEMC resources as necessary. 


\subsubsection{Progress Summary for Task 1}

Work on this task started in December 2011. Much of the early work was performed by the team from the Illinois Institute of Technology under direction of Prof. Zongzhi Li. Prof. Li was working with a team of 6 students on both the RTSTEP project as well as an independently funded project for FHWA that IIT is working on in collaboration with the City of Chicago Department of Transportation, the Chicago Metropolitan Agency for Planning, and other collaborators. Before their involvement with TRACC as a subcontractor under the RTSTEP project, the IIT team focused on improving and validating the Chicago Business District model. Staff at TRACC defined individual work assignments that were implemented by students from IIT to address high priority network editing. The team at IIT also worked on the adjustment procedures for Origins and Destinations in the original demand model, as well as calibration of the traffic assignment process for normal days. This work formed necessary foundation for RTSTEP due to the fact that the normal day is in fact the starting point for deviations caused by evacuation trips.

AECOM was also heavily involved with this task. A work planning meeting was held in January 2011 on defining individual responsibilities, and AECOM was working on the conversion of the underlying data to the new TRANSIMS 5 format. TRANSIMS 5, which was still under development at the time, has also been modified to better perform as part of the RTSTEP application. Throughout the project, AECOM was developing the underlying software library that drives the individual tools that TRANSIMS consists of. This library called SysLib will be frequently mentioned throughout this report. The library serves the purpose of standardizing the underlying software approaches and objects to make higher level programs more effective and less error prone.

By March 2012, TRACC, AECOM and IIT finished much of the work on the network supply model, correcting insufficiencies and introducing a number of network enhancements. A large number of modifications were made to the network. The sections of I-294, 1-90, I-290, I-355, I-88, I-57, I-55, I-65 and $1-80$ have been realigned to match the ortho-imagery. Missing ramps and loops were added to replace simplified straight links from the original CMAP network. A new feature for the NetPrep tool was implemented at TRACC. This feature allows to read in the Open Street Map (OSM) network data and to generate a routable TRANSIMS network from this open source project. The TRANSIMS network was modified based on the OSM data. Specifically, the nodes which correspond to arterial road intersections were aligned to match those of the OSM data. Also, the Chicago Business District (CBD) area signal timing and phasing data originally prepared by IIT for the version 4 model of TRANSIMS was converted into the new version 5 format.

To obtain an accurate traffic assignment result, it is important to mimic the real life transportation network details as detailed as possible. Significant effort was spent on refining the locations of activity locations (the abstract equivalent of homes and work locations, or rather the start and end locations of all trips that are simulated in a given model) and intersection controls (such as traffic signals and stop signs). Activity locations (the trip loading and unloading points) are spatial locations that represent dwelling units, employment locations, social and recreational facilities, and shopping centers. These locations either produce or attract trips. Activity locations were synthesized based on the area type (business district, rural area, etc.) and facility type (highways, major and minor arterials, local streets, 
ramps, etc.). In some situations, the activity locations were not generated properly using the default settings in TRANSIMS. For example, there were no activity locations generated on Lower Wacker Drive, while there are quite a few loading docks and parking spots located on that street. Some of those changes were automated and some required manual tweaks. Also the traffic assignment process had to be calibrated. Traffic assignment is the process of calculating a path for each of the travelers on the network based on the current understanding of congestion patterns. For the validation of the model assignment result, field link counts were used. The TRANSIMS Router, along with several other TRANSIMS utilities, was used to perform the assignment. To mimic real live traffic volumes, several of the TRANSIMS Router attributes were adjusted. For example, facility bias factors and distance values were adjusted. Those parameters are specific to a given geographical region. Facility bias helps modeling a situation in which a traveler does not necessarily choose the fastest route but rather prefers to take a highway, even if it takes longer.

The RTSTEP team also worked on increasingly accurate models of traffic signals. In a transportation model, it is very important to replicate real life traffic signal timing and phasing because the transportation network is a highly sensitive dynamical system. This means that a small change in the intersection control timing and phasing may lead to large changes of traffic patterns in a relatively large area. However, it is impractical to manually input the information for all of the traffic signals in the model region; there are 3000 signals alone within the limits of the City of Chicago. A more practical approach was to manually code the traffic signals for "critical intersections." Those are intersections that are part of evacuation routes as well as intersections with large traffic volumes under normal circumstances. There are approximately 430 of those intersections in the model area covering the Chicago metropolitan region. Unfortunately, intersection control data is currently not available in machine-readable format but rather available as engineering documents scanned into PDF files. TRACC along with AECOM developed an Excel tool which streamlined the process of converting the data into the TRANSIMS format. The tool also performs quality control and helps to minimize human error.

To address the need for model validation, the AECOM team established quality control procedures for the process of extracting traffic volumes from CMAP's EMME2 network and used this to validate the TRANSIMS Router assigned volumes.

Work on converting and testing the traffic signal locations as well as their timing and phasing was completed in June. It was determined that there is no clear pattern to distinguish whether an intersection is controlled by a traffic signal versus a traffic sign. Thus, it was shown that this process could not be fully automated, i.e. the location of traffic signals could not be accurately synthesized at a reasonable error rate. The group from TRACC and AECOM developed a list of actual traffic signal locations which also contains the cycle length for each of the signals and developed a program to convert this data into the TRANSIMS format. The TRACC team also validated 300 of the traffic signals manually coded by IIT in the central business district. A number of shortcomings were fixed. Several short links (length $<18 \mathrm{~m}$ ) in the highway network were modified or deleted to prevent problems in the Microsimulator, one of the TRANSIMS tools. Due to the design of this simulation algorithm, the traffic flow could not be accurately estimated on links shorter than $18 \mathrm{~m}$. Some of those short links 
corresponded to real live links and were artificially modified; some of those were coding errors from the previous version of the network and were removed.

Finally, toll data was refined in the normal day model for the metropolitan region. In the previous revision, the tolls were the same for trucks and passenger cars, while they are different in real life. Previously, there was no distinction between express (for I-PASS users) and cash lanes. The accurate representation of toll stations on the network allows predicting path choices as well as to estimate the traffic density and speed on the toll links more accurately. A good estimation of "normal day" driver's behavior affects travel patterns during an evacuation period. The group from AECOM developed and documented the network conversion process for the Chicago Metropolitan Are network. The set of scripts and documentation was developed which would allow replicating the process for a different geographical area. Usually, network conversion is a very time consuming process. Keeping in mind that the potential customers of the RTSTEP toolbox are local transportation or emergency management authorities which usually are very limited in terms of man power, the new process will allow preparing network data while having very limited resources.

\subsubsection{Task 2: Development of Tools to Evaluate Evacuation Routes and Strategies}

An important task within the overall RTSTEP project is the modeling of evacuation routes. TRANSIMS has been developed as a general purpose traffic forecasting tool and does not distinguish between normal roadways and designated evacuation routes at present. Nevertheless, the underlying components are readily available in TRANSIMS, such as the capability of prohibiting turns by time of day, preempting and manipulating traffic signals, closing and reserving lanes for specific types of vehicles, and much more. Lane reversals, such as those implemented during counter-flow evacuation scenarios, can be readily modeled and are already a standard feature of TRANSIMS. The work in this area focuses on the development of a tool that allows emergency responders to define the characteristics of emergency evacuation routes with a degree of flexibility and automation.

\subsubsection{Key Deliverables}

- Determination of the procedures used by emergency responders to implement the establishment of emergency evacuation routes. Discussion of findings with OEMC and emergency response planners to set the priorities for implementation of modeling tools.

- Development of tools to configure evacuation routes with a reasonable degree of simplicity and to run TRANSIMS on the basis of the resulting modified network.

- Development of tools to evaluate rail options, to allow for capacity planning, and to determine the impact on target communities.

- Documentation of the tools and training of emergency response planners in the use of the tools

\subsubsection{Progress Summary for Task 2}

At the beginning of the project in December 2010, TRACC staff developed a short document titled "'RTSTEP Technical Proposal" that was discussed in detail at semimonthly team meetings. This document described the anticipated software development needs for RTSTEP, both in an idealistic way (e.g. what the tool would look like under ideal circumstances, with unlimited funding, and with 
unlimited implementation time), as well as under the existing time and funding constraints of the RTSTEP project. The approach served TRACC well in the past - taking a step back from considering only immediate needs and allowing for a more consistent and more extensible development approach. The main issue was weighing the cost of the implementation of complex graphical inputs and representations against the more simple programming approaches. The goal was to create a user interface that is open for future enhancements and will not limit itself to a few features of immediate urgency.

As the proposal was discussed, it was found reasonably feasible. Some of the work was directly related to TRACC's visualization work, while other components were related to the development of the underlying TRANSIMS tools by AECOM. Another design goal of the RTSTEP Technical Proposal was the optimization of workflow between the geographically separated developers, and to more clearly delineate individual components and their interactions. The appropriate methodologies also depended heavily on the target user community. It is difficult to precisely define the typical user of the final product, but it was clear that emergency responders needed to be involved early in the project to ensure that the software would meet their needs. From the perspective of a software developer, the needs of emergency responders and evacuation planners are also difficult to predict, and it was important to start the dialog early in the process of establish such a project. At Argonne, TRACC participated in a number of discussion groups and expert panels on the scientific aspects of national security issues, and TRACC. TRACC staff is also on the transportation subcommittees of both the Illinois Terrorism Task Force and the Regional Catastrophic Preparedness Teams. Having had access to emergency response planners from different organizations, such as IDOT, CTA, PACE, the Red Cross, the State Police, and many other organizations helped to create appropriate feedback during the development of the RTSTEP application.

TRACC staff also performed literature research, and prepared presentations on the underlying technologies to a number of groups in the Chicago Region. TRACC was also looking at the effects of improvised nuclear devices on the transportation infrastructure as part of an ongoing effort in the Chicago region to be better prepared for IND events.

A major effort under this task was the development of the EvacNet tool as part of the TRANSIMS toolbox. The EvacNet tool has three main functions with regards to modifying the highway network for emergency evacuations: (1) establishing turn prohibitions; (2) enforcing lane use restriction; and (3) evacuation route planning. The three main functions require three input files. New formats and input functions were designed for the EvacNet input files. The turn prohibition function prohibits use of a turn for certain vehicles for a given time period during the evacuation. The input for this function is called Evac_Turn_Penalty'. The lane use restriction function is to restrict lane use on a link for certain vehicles for a given time period during evacuation. The SysLib data structures were utilized for Evac_Lane_Use and Evac_Turn_Penalty. Evac_Route has been defined its own data and file structure in EvacNet.exe showing below. 
EvacNet.exe is capable of reading all supply elements of a normal day network and then output them to the 'EvacNet' directory, which forms the base network for case studies. Network supply changes related to evacuation are saved in this particular folder. A new control key for Evac_Routes file was created, called EVAC_ROUTES_FILE, which can be used in the EvacNet.ctl control file. According to the Evac_Routes input, EvacNet.exe changes the normal day model's signal plan, timing plan and phasing plan. A set of candidate evacuation routes was defined as part of this approach. This set of evacuation routes includes most of the freeways and a large number of arterial roads. The team refined the candidate routes and improved the connectivity of the evacuation routes network to create more realistic evacuation simulation capabilities.

Laying the foundation for the behavioral evacuation demand model, EvacNet was updated to include links affected by individual scenarios, and the development of the evacuation response survey intended to provide information on how individuals respond in various evacuation scenarios. Two IIT students, Yifang Liu and Fei Qu, worked closely with Kuilin Zhang to update the candidate evacuation routes set and the inputs for EvacNet, testing the functionality of EvacNet, and defining segments for both freeway and arterial corridors for the heuristic rule based evacuation network design procedure.

The EvacNet tool is capable of not only identifying stretches of road dedicated to evacuation routes and access points for those routes, but also computes the traffic signal timing and phasing to optimally accommodate evacuation traffic. There are two distinct types of intersections - intersection of two evacuation routes, and intersection of an evacuation route and non-evacuation route. For the intersection of two evacuation routes, the flow is allowed on both adjunct roads, and for the latter case only the movements associated with evacuation traffic has green phases assigned to them. In order to allow off-ramp exits, lane use restrictions for evacuation vehicles only in evacuation zones were included, and both lane_use and turn_penalty were updated to the latest version of SysLib., the central TRANSIMS software library The evacuation network plans for the Wrigley Field case study were refined and scenario tests for the proposed evacuation scenarios were conducted.

\subsubsection{Task 3: Tools to Model Populations, Evacuation Trips and Background Traffic}

TRANSIMS, just like any other traffic simulation package, cannot readily predict the destinations of evacuees who are able to evacuate by car or transit. Identifying likely destinations is clearly a pressing issue for emergency responders, and a large part of the work will focus on the implementation of tools that simplify running the models under different assumptions to test and compare the responses of the transportation network to varying demand. Evacuation trips also do not exist in a vacuum; a significant portion of the traffic on the road may be driven by normal day demand, such as trips to work by people outside the affected areas. Thus, this task also focuses on creating a robust normal day model for the Chicago Metropolitan Area that forms the basis for all evacuation models.

\subsubsection{Key Deliverables}

- Evaluation of currently available methodologies for the modification and synthesis of evacuation trips and the compatibility with and impact on the existing TRANSIMS model. 
- Development of tools that allow for the creation and modification of evacuation trip destinations. This will also affect the origin of these trips and their start times, in particular for evacuees that either live in the affected area or that have already arrived there.

- Development of algorithms and tools to increase the fidelity of the simulation and to resolve problems caused by some artifacts of the TRANSIMS approach that cause poor fidelity of the simulations in highly congested areas.

- Evaluation and subsequent implementation of building egress models and pedestrian simulation as necessary to increase the fidelity of the model in areas where evacuation on foot will be the most likely mode.

- Integration of the modified trip and activity data with the TRANSIMS model to simplify parameter studies and the comparison of different scenarios and assumptions fed into the process by emergency preparedness planners.

\subsubsection{Progress Summary for Task 3}

A new tool called EvacDemand was proposed by TRACC to be developed as a part of TRANSIMS. TRACC staff developed detailed specifications for the features required to be implemented within the new tool. One of the proposed features would allow the visualization software TransimsVIS to be linked with EvacDemand through an interface. This allows the modeler to select and modify evacuation-related demand parameters right on the map. For example, it is possible to modify origins and destination of evacuation trips, and to assign weights to certain activity locations to make them more or less attractive. Users are able to specify a time span for trip start times for each of the origins. The proposed tool was discussed in detail during bi-weekly project progress meetings.

A literature review was also performed to learn how other models in the past have handled demand modification under evacuation scenarios. The literature review was also looking at surveys that have been conducted to get evacuation responses, including response time, compliance rates, destination types, etc.

A prototype of this tool was developed by March. The work for scenario specifications was also finalized and then integrated with the Visualizer (developed under Task 4) as well as EvacNet. The team worked on designing the underlying behavioral model which forms the basis for the modeling response of the evacuees under simulated conditions.

For calibration of the behavioral models that have been developed, the TRACC team decided to prepare and perform an evacuation decision survey. A random sample of a population from the Chicago Metropolitan Area was surveyed. The goal was to collect basic demographic information on the responders, as well as the location data of all members of the households. The second part of the survey presented one of several scenarios to the participant, and asked for the participant's anticipated response to that scenario.

By April, the team improved the evacuation network design tool EvacNet, refined candidate evacuation route sets for both freeways and arterials, and designed a heuristic rule based procedure for defining evacuation network plans for given extreme event sites, shelter locations, and budget constraints. Two 
students from the Illinois Institute of Technology, Yi Liu and Xi Lu, worked closely with Kuilin Zhang on refining candidate evacuation route sets, preparing the inputs for EvacNet, and defining the key rules for the heuristic rule-based evacuation network design procedure.

The evacuation network plan design is essentially a network redesign problem. In this study, a set of candidate evacuation routes was defined. Given a set of candidate routes, extreme event sites, evacuation demand, and corresponding shelter locations, the evacuation network plan design problem finds a subset of optimal evacuation routes, to determine corresponding traffic management and operational strategies (e.g. turn prohibition, lane use restriction, contra-flow lane, and signal re-timing) to maximize network throughput for evacuation zones or minimize clearance time, with minimum impacts to the normal demand satisfying a set of capacity, budget, and time constraints. The main decision variables for designing the evacuation network plan in this study are the accessibility of intersections from other approaches, lane use restriction, and turn prohibitions.

EvacDemand allows evacuation analysts to input evacuation scenario information and baseline travel information and then modifies the baseline travel information to respond to the evacuation scenario specified. By May, the following capabilities were implemented: creation of demonstration scenarios, incorporation of final network data into scenarios, and completion of the evacuation simulation modeling process. Work has also continued with the development of the evacuation response survey intended to provide information on how individuals respond in various evacuation scenarios. The survey design was finalized and pilot testing began in May to improve the survey in response to pilot respondent suggestions. Since the approach of using maps is considered innovative, the National Opinion Research Center researchers suggested developing quick video tutorials on how to answer those questions which ask a respondent to specify a location on a map.

In order to improve the fidelity of the demand model, a microscopic model of the event locations has been built by the research team from NIU. The information of three locations of interest (Wrigley Field, Sig Flags and Chicagoland Speedway) was collected and analyzed. The annual number of visitors at Six Flags was obtained as well as collecting the land use, employment, and demographic information of the surrounding area. Capacity and similar demographic and employment information was collected for other locations. In addition, the NIU team analyzed the structure of Wrigley Field as well as locations and characteristics of each of the entrances/exits in the Field. The number of seats per block and possible flow at the exits was estimated. The software chosen for this analysis, after evaluating eight different software solutions, was LEGION. An AutoCAD model of the Wrigley stadium along with the model of Sig Flags Great America was created. Models of both of the locations were used in the LEGION simulator to get preliminary results on the behavioral model of the people during the evacuation.

\subsubsection{Task 4: Development of Visualization Methodologies to Compare Scenarios}

The massive amounts of data resulting from TRANSIMS runs are not easily interpreted without the help of powerful visualization tools. TRACC has developed significant contributions to TRANSIMS visualization techniques and developed additional tools as necessary to present results in a meaningful and easy-touse fashion to emergency response planners. The applicability of different visualization strategies was determined as an early part of the RTSTEP project and is an integral part of much of the scope of work. 


\subsubsection{Key Deliverables}

- Development of visualization concepts, both static and in form of videos, that will suit the needs of emergency response planners and illustrate the differences between given scenarios effectively.

- Implementation of the necessary software and tools to create such visualizations both in batch mode and with a certain level of interactivity.

- Documentation of the tools developed under this task. Training in the effective use of the tools.

\subsubsection{Progress Summary for Task 4}

RTSTEP has a need for both a tool which can be used to configure transportation management options for evacuation studies as well as a tool which can visualize the final results of the evacuation case studies. The original TransimsVIS software was developed by TRACC to aid in the visualization of generic TRANSIMS results. The tool was pseudo-3D, python-based, and semi-interactive - it included many features including visualizations of: vehicle trajectory, regional congestion, regional signal patterns, transit ridership, vehicle paths, car following, vehicle speeds, and more. It also included features such as movie creation, waypoint selection for cinematic camera movements, and side-by-side comparisons. But there were many motivations to re-code a new version of the visualizer for use in the RTSTEP project, based on TRACC's capabilities and the ready availability of high-end graphics cards on typical desktop computers.

The development of TransimsVIS 2.0 proceeded through a number of phases. First, the basic program structure was designed and the integration with TRANSIMS version 5 was conceptualized. Coding began with TRANSIMS integration followed by a framework for performance testing. The next development was the navigation framework, followed by the visibility testing algorithms. This was followed by a network drawing algorithm and all the corresponding optimizations. After this, the ortho-imagery algorithm was designed as well as a general framework for multithreading. It also includes a comprehensive optimization of each feature to ensure perfect performance on even slower machines and an attempt to run on specialized hardware such as the mini lambda system at TRACC. The mini lambda system is a synchronous multi-tile high resolution display system, which was constructed to improve the modeler's capabilities in network editing, visualization, and analysis. It has been used for high end demonstrations throughout the project.

A major development was for the TransimsVIS software to support evacuation visualizations. An important part of this work was the development of a compression algorithm for vehicle data. Since the execution of regional simulations for every second of the day is very expensive from a disk storage perspective, this compression scheme cuts that space requirement down by a factor of 4 or more. Along with the vehicle compression algorithm, a multithreaded method was developed that can load vehicles from the disk drive to the visualizer very efficiently; the method resembles a web video which has a similar buffer. This procedure is combined with a vehicle rendering step that computes the geometry of all vehicles loaded. The development also included data plot visualizations. 
Of pressing concern was adjusting the software to handle the regional demands of the Chicago network. With over 27 million daily trips and covering over 10,000 square miles, the Chicago Metropolitan Area pushes any visualization software to the limit. This limitation required upgrades to the vehicle loading procedure, data plot computation procedure, and the ortho-imagery loading routine. In addition to these upgrades, the framework was put in place to identify model elements (initially just links and nodes), and display their attributes. Finally, it was important early in the project to start testing the software among the RTSTEP group to identify bugs and to gather more comprehensive feedback. This involved establishing a new branch of the TRANSIMS source code control archive and updating the software to run on more generalized input data.

The software features also a waypoint system and an interpolation feature. In addition, a large degree of restructuring was done half way through the project that doubled performance for drawing the data plot, and increased vehicle trajectory visualization speed by $20 \%$. It cut overall memory usage by $30 \%$ as well. The restructuring paved the way to easily incorporate new visual elements specific to evacuation visualizations.

In May, development continued on the TransimsVIS software to support evacuation visualization. It included four major feature additions: probe vehicles, improved data plot intelligence, 3D vehicle models, and additional satellite imagery. Development also began on the link plot feature, which is a mesoscopic visualization aimed at communicating information which is relevant at the link-by-link scope.

Along with changes in the TransimsVIS, one of the Microsimulator's output data files was modified. Snapshot file fields were expanded to include waiting time, time difference, and user data fields in the compressed and standard output. The simulation plan data was expanded to include activity duration and leg schedule time. The Microsimulator tracks the difference between the scheduled time and the actual time at each time step. All of those extra pieces of data in the Snapshot file are to be used for visualization purposes to support the evacuation case studies effort.

By late June, development culminated in the TransimsVIS software to support evacuation visualizations in a very comprehensive manner. It included new link plots and 3D vehicle models while zoomed in. In addition, it included several new features: 3D building models, strategic routes visualization, and evacuation area visualization.

The final version of the tools allows for several different modes of operation, both to configure and to evaluate evacuation scenarios. All available ortho-imagery from USGS and other sources was layered into a single large scale mosaic to make a complete map available across the entire metropolitan region. Heat plots and bar graphs on top of roads allow for comprehensive analysis of aggregate information, while the addition of a generic GIS capability allow for the inclusion of user-provided data sets to enhance the modeling capabilities. 


\subsubsection{Task 5: Integration of the Transportation Model with the Dispersion Model}

TRANSIMS has the unique capability to consider the movements of individual travelers through and around a contaminated area. This capability makes it significantly easier to determine the exposure of individuals to radiological hazards because of their accumulative nature. Without a transportation component, the determination of the level of exposure would be limited to the assumption of a statistically immobile population. With TRANSIMS, the radiological exposure can be aggregated on an individual personal level while moving on complex paths through the time-dependent dispersion cloud, whether by train, bus, or walking or in a personal vehicle.

\subsubsection{Key Deliverables}

- Development of strategies for integration between a dispersion model and RTSTEP. Evaluation of methodologies and determination of the best feasible approach. Discussion with OEMC on the level of integration.

- Modification of TRANSIMS tools and development of utilities to implement the integration. Establishment of a framework that can be used by modelers to set up specific scenarios and start the necessary TRANSIMS runs to obtain results.

- Documentation of the tools developed under this task. Training in the effective use of the tools.

\subsubsection{Progress Summary for Task 5}

The National Atmospheric Release Advisory Center (NARAC) of Lawrence Livermore National Laboratory (LLNL) provides operational services, tools, and expertise for preparedness, response, and recovery from dispersion events. The services include dispersion event modeling, analysis and guidelines for response actions. It can be a valuable resource for various event information including weather data, hazardous substance, geographic and demographic data, and other measurement and observations. TRACC requested access to this system to simulate dispersion events and to integrate them with the RTSTEP project. This work was originally to be performed in a companion project between the City of Chicago and Argonne, but it was decided to delay this project.

TRACC staff then investigated several packages available on the market for computational fluid dynamics (CFD) modeling. To develop a meaningful interface to RTSTEP, TRACC explored the use of CFD and other dispersion modeling tools. The exploration of dispersion modeling tools should lead to generic interfaces that can be replaced in the future with more specific interfaces as needed.

The size of the problem, which arises while modeling plume dispersion, is of significant size. It was initially decided to explore the STAR-CCM+ CFD code for modeling of a small region surrounding the source. STAR-CCM+ was chosen because TRACC has an in-house expertise with this package. It was decided to also take a look at mesoscopic modeling tools for the rest of the domain. The meso-scale models predict large grain atmospheric conditions over a large area.

To address the computational challenges, an initial computational fluid dynamics (CFD) model has been developed for a small urban area. This helped with developing and verifying various modeling methods and capabilities. The model developed consisted of a small urban area with generic building geometries. 
The area was $120 \mathrm{~m} \times 120 \mathrm{~m}$. Analysis was done on a volume of fluid (air) surrounding the buildings. To perform the finite element model (FEM) analysis, the volume was meshed with a polyhedral volume mesh. The simulation model was solved numerically. The simulation model was described by a boundary value problem with the following boundary conditions: East, Top = pressure outlet, North, South = symmetry outlet.

By April, the focus of Task 5 was to develop a steady-state air flow model. First, the steady-state air flow model was being developed, providing an initial condition for the subsequent plume dispersion model. As discussed above, those models are limited in terms of the size of the domain, but are a good start and can be a good starting point for surrogate mesoscopic dispersion models.

The steady-state model means that the dispersion is time independent and some averaged solution is considered. Since the plume generation is expected to vary in time, it is necessary to develop an unsteady flow model and perform transient analysis. This is accomplished by Star-CCM+, redefining the model as unsteady flow model, and associating the boundary condition with a user function for time varying flow speed at the plume inlet.

With the addition of the gaseous component and solid particle phase, the simulation model was also solved in an unsteady state mode. The multi-component, multi-phase dispersion model was further refined by including the effects of gravity, earth boundary layer, and particulate restitution factors.

To scale the modeling capabilities up, a set of tools was developed for constructing 3D models of the buildings based on their foot print geometry and approximate height based on data provided by OEMC. The extrusion from footprint data created urban canyons with an approximated geometry of all buildings within the city limits. It allows not only visualizing all of the building in TransimsVIS, but also using the models in the computational fluid dynamics software to predict gas flow with in a large domain which includes all of the buildings. The TRACC team identified a meshing scheme and flow parameters which lead to the conversion of the computation methods for calculating flow of methane and aluminum dispersed through a rectangular window inside the domain.

As a case study, a plume dispersion model was developed for the Wrigley Field area. Using python scripts, the buildings in the Wrigley field area of 3 or more stories were created. As part of the final scenario, a series of reference models were developed. For the application to suburban areas, a volume measuring $3.2 \mathrm{~km}$ by $3.2 \mathrm{~km}$ by $2 \mathrm{~km}$ was created with no buildings. The plume inlet was centered $200 \mathrm{~m}$ from the wind inlet. Originally, the inlet was a cylinder due to the problems with the sphere inlet in the Wrigley simulations. With no buildings in this model, the surface wrapper was no longer necessary.

Concurrently, TRACC continued to evaluate additional software tools with mesoscopic modeling capabilities. TRACC obtained a copy of the HPAC toolbox (Hazard Prediction and Assessment Capability) developed by the Defense Threat Reduction Agency (DTRA). The TRACC team tested the HPAC software from DTRA. In contrast with the CFD models HPAC uses "rough" aggregated statistical models for predicting the impact of an explosion. However, the advantage of using HPAC is the amount of computing time needed is much less compared to CFD calculations as well as availability of predefined parameters for different type of threats. 


\subsubsection{Task 6: Case Studies}

Significant effort is needed for the development of a few representative case studies that highlight the methodologies used and the capabilities that result from the RTSTEP project. The case studies will be defined in greater detail early in the project in consultation with emergency response planners and OEMC. The scenarios defined during the 2009 RCPT Summit in Chicago (July 15 and 16, 2009) will serve as the basis for these developments. The scenarios will also form the basis for training materials and training courses planned to transfer the technology to emergency response planners before the end of the RTSTEP project.

\subsubsection{Key Deliverables}

- Detailed definition of the postulated events, determination of likely response strategies with regards to transportation, and determination of the need for additional data.

- Determination of the need for model refinements to best addresses the questions to be answered by the case studies. Determination of the scope of road and transit network refinement in the affected areas.

- Determination of likely destinations for evacuees, the establishment of mass transportation, and the location and capacities of emergency shelters that will be used.

- Detailed results from case studies performed as part of this work, such as data, graphs, animations, and presentations.

- Detailed documentation of the processes, assumptions, methodologies, and limitations of the scenarios developed as case studies.

- Training materials based on the case studies that are suitable for teaching the methodology to emergency response planners to be identified by OEMC.

\subsubsection{Progress Summary for Task 6}

A significant number of changes were implemented within the Microsimulator tool. Those changes more realistically simulate heterogeneous traveler behavior. Facility-based traveler behavior keys were changed to traveler-type based keys, and a facility biased factor was added. Those changes allow simulating different driver behavior based on the facility type. For example, the car following distance is generally larger on a highway compared to an average car following distance on a minor road. More traveler types using nested logic were enabled, and the use of a unique random number seed for each traveler was implemented. This feature will allow distinguishing driver behavior by type. For example, an aggressive driver tends to change lanes more frequently compared to a less aggressive one.

During the month of February subarea processing (unsimulated, macroscopic, mesoscopic, and microscopic) was implemented. This is an extremely important feature for modeling a large scale transportation network because it is impossible to build a high fidelity model of the network supply for the entire region. It is associated with a tremendous modeling and computing effort, and is not cost effective. This feature will allow using mesoscopic or macroscopic simulation for remote areas which do not impact the transportation network performance significantly and thus are not required to be 
accurately modeled and simulated with a microsimulation technique. This approach saves development time as well as speeds up the computing time necessary to run a model on a computer.

The Microsimulator was then extensively tested and many bugs were corrected. Lane swapping logic was added which allowed improving the accuracy of the simulation results. The Router and PlanSum were enhanced to improve consistency between Router and PlanSum link delay files. Plan processing routines were enhanced and reports to summarize and compare travel times and trip lengths were added. Data conversion routines were enhanced to convert plan, pocket lane, and signal timing information accurately. Data processing and conversion tools for transit networks, count and volume data were enhanced.

A large number of changes were made in the core TRANSIMS executable. The major improvement is the implementation of the Message Passing interface (MPI) capabilities that were added to several key executables such as the Router and PlanSum. This allows utilizing massively parallel computers with distributed memory (when separate compute nodes are connected with each other through a network). The TRACC cluster is an example of such a machine. The new MPI implementation was tested and debugged. Other major changes were done in the Microsimulator. Various Microsimulator algorithms were tested and Microsimulator performance was improved. Calibration keys for facility type and traveler type controls were added to Microsimulator. The Microsimulator's driver behavior and data structures were improved. Parallel to improving the performance of the code, different compilation procedures was tested on the TRACC cluster to identify the optimal combination of compiler and optimization flags. Proper compilation of a code into an executable is crucial and can save hours of computing time for a single model run.

The TRANSIMS software was modified so that the lane use restrictions can be applied based on a vehicle type. For example, it is possible to model a lane on a stretch of road which is dedicated to emergency vehicles or model lanes which can be used only by IPASS users. The traveler type usage within the ConvertTrips tool was enhanced. Now a modeler has more control of how a traveler type is to be assigned to a trip. For example, as an input to the model, a number of vehicle trips from zone $A$ to zone $B$ are given. The mode of transportation and departure time is also part of the input. ConvertTrips based on the trip attributes and a distribution of a random variable assigns a travel type to each of the trips. For example, travelers who use in-vehicle GPS navigation could be distinguished from those who do not. Different traveler types would choose different paths. For example, an IPASS user is more likely to take a toll road compared to the traveler who does not have an IPASS. The capability to control assignment of traveler types on to trips brings an extra level of accuracy in to the model.

A new simulation algorithm was implemented within the Microsimulator tool. The new simulation algorithm is a macroscopic queue-based algorithm. The name macroscopic comes from the fact that the traffic flow model is based on macroscopic properties, like the relationship between vehicle density and average speed on a link. The disadvantage of the new algorithm is that the lane changing mechanics are not modeled. On the other hand, the data requirements as well as run time is significantly less compared to the previously used mesoscopic simulation algorithm. The run time is 10 times faster when the mesoscopic simulation technique is used. 
Significant effort was spent on the MPI version of the Microsimulator. Logic was added to set and restrict the partition range considered by MPI machines. MPI communications, data structure packing and unpacking, and machine specific network data partitioning were implemented. Travelers, vehicles, and boundary link occupancy data transfers between MPI machines was implemented and boundary issues were addressed.

The TRANSIMS software was modified so that the modeler has more control on selecting and identifying travelers whose plans go through a certain geographical area, for example through an evacuation zone. The MPI framework for the simulator module was also improved. When an MPI environment is used, the transportation network is partitioned and every individual machine is "responsible" for one of those partitions.

Some of the core data structures were modified. The changes allow for a friendlier user interface to model lane restrictions on the network. A new tool called Relocate allows a user to seamlessly move transportation demand data from one network revision to another. This makes the process of synchronizing travel plans and network data much easier. A large number of bugs identified during case studies in the Router box were fixed. The new version of the Router allows obtaining more stable and consistent results. The logic in the traffic simulator was modified and enhanced. This allows eliminating some of the factors which lead to an artificial congestion on the network. Transit vehicles can now be simulated. The new routing algorithms for transit have been implemented in August. This allows having a full scale transit model. The software compilation scripts were moved to and tested on the TRACC cluster, which is more reliable compared to the machines used at AECOM.

The case studies concentrated in this study on the development of the software and in the evaluation and implementation of features necessary to create a tool that is suitable for use by emergency responders. Particular scenarios that are described in great detail in section Error! Reference source not found. of this report include Wrigley Field, Naperville, and Six Flags Great America. The tool was developed to configure different scenarios in any area in the region, and the chosen scenarios are highlighting different aspects of the modeling needs.

\subsection{Network Coverage by County}

The overall extend of the model area is shown in Figure 7.1. It covers approximately 10,000 square miles, and stretches from Milwaukee in the North to Kankakee in the South, and Rockford in the West to Michigan in the East. The network is a modeling network that includes all important streets in the model area, and is more refined in densely populated places.

The maps on the following pages provide a basic understanding of the highway network as it is used in the underlying RTSTEP and TRANSIMS models. Figure 7.1 shows the model network in red. The figure illustrates the general topology of the network, showing that the model area is largely bounded by some major exit points along the Interstate highway system. Cities and towns on the boundary of the model area are modeled relatively sparsely. As a consequence, the details for smaller towns like Kankakee may 
be far more detailed than the details for Milwaukee. Coverage for the 16 Counties in the Combined Statistical Area are excellent, as demonstrated in the maps in this chapter.

Data layers such as Hospitals, schools, police stations, and fire stations have been provided by CMAP, OEMC, IEMA, and GIS experts from the different counties. An example for enhanced data provided by a county is shown in Figure 7.11 and Figure 7.12 for Kankakee County.

The maps in this chapter have been created using the ArcMap software from ESRI, a tool commonly available to GIS departments at participating counties. Layers are provided as part of the RTSTEP application. 


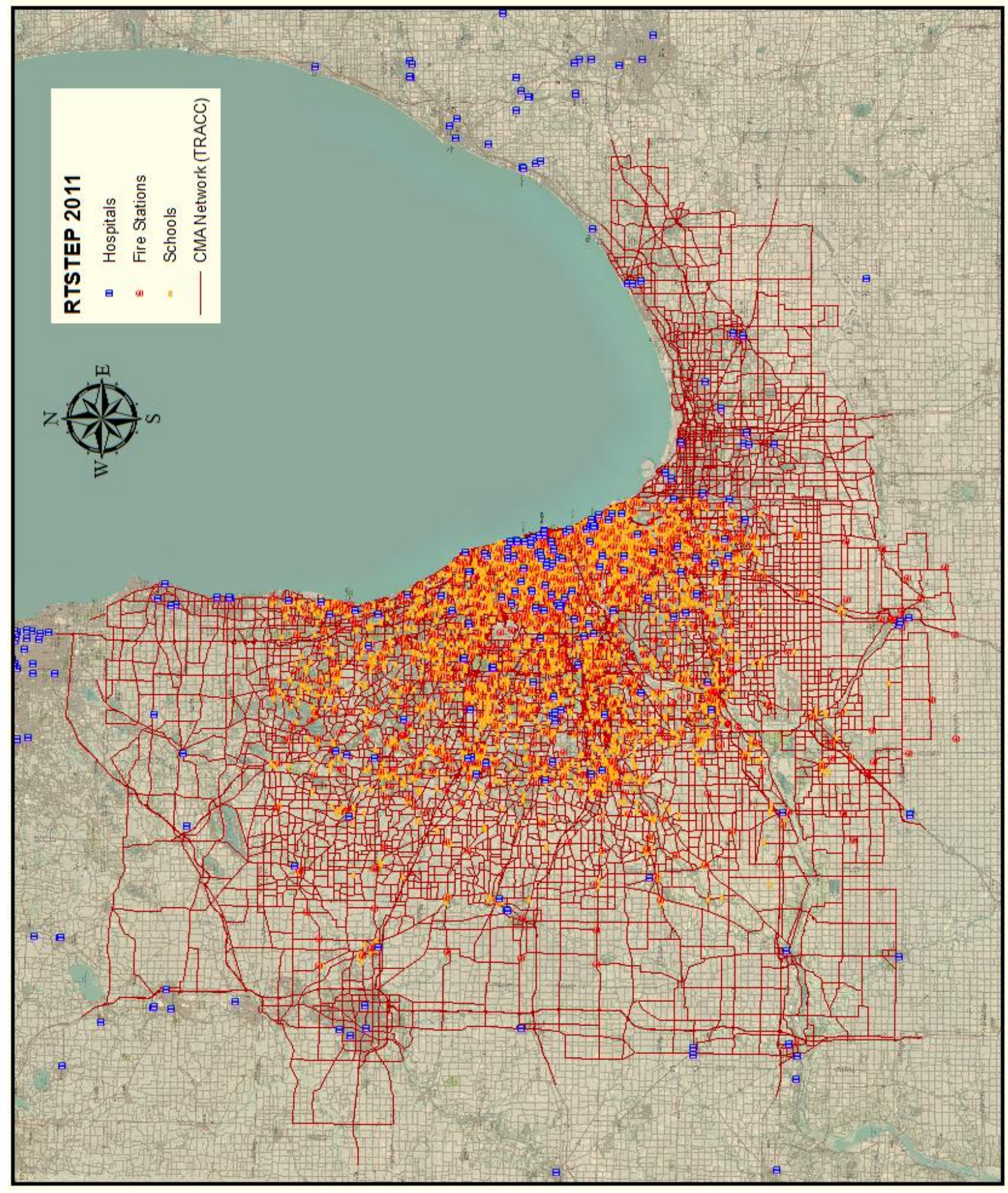

Figure 7.1: Extent of the Chicago Metropolitan Area highway network used for RTSTEP 


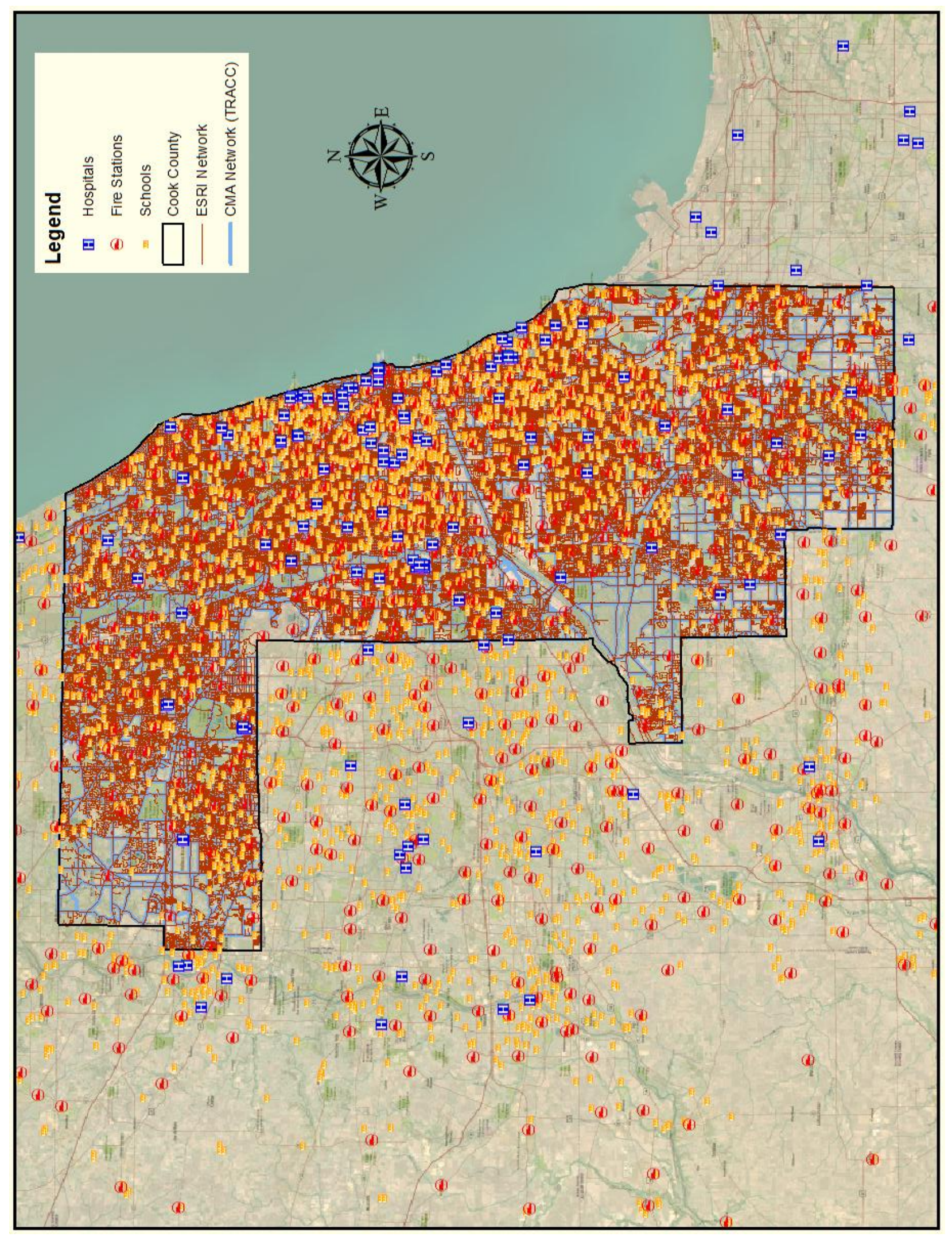

Figure 7.2: Cook County 


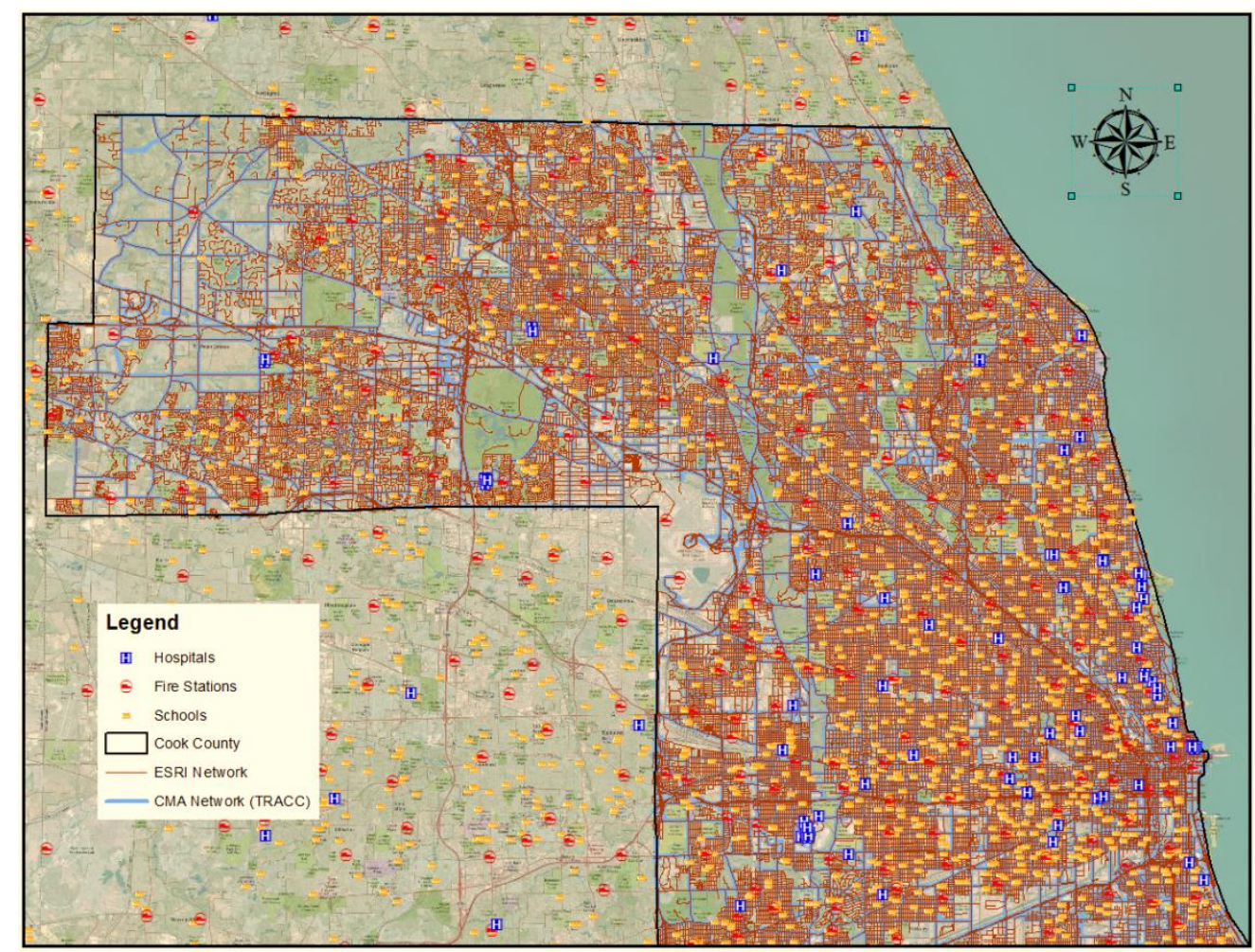

Figure 7.3: Northern Cook County

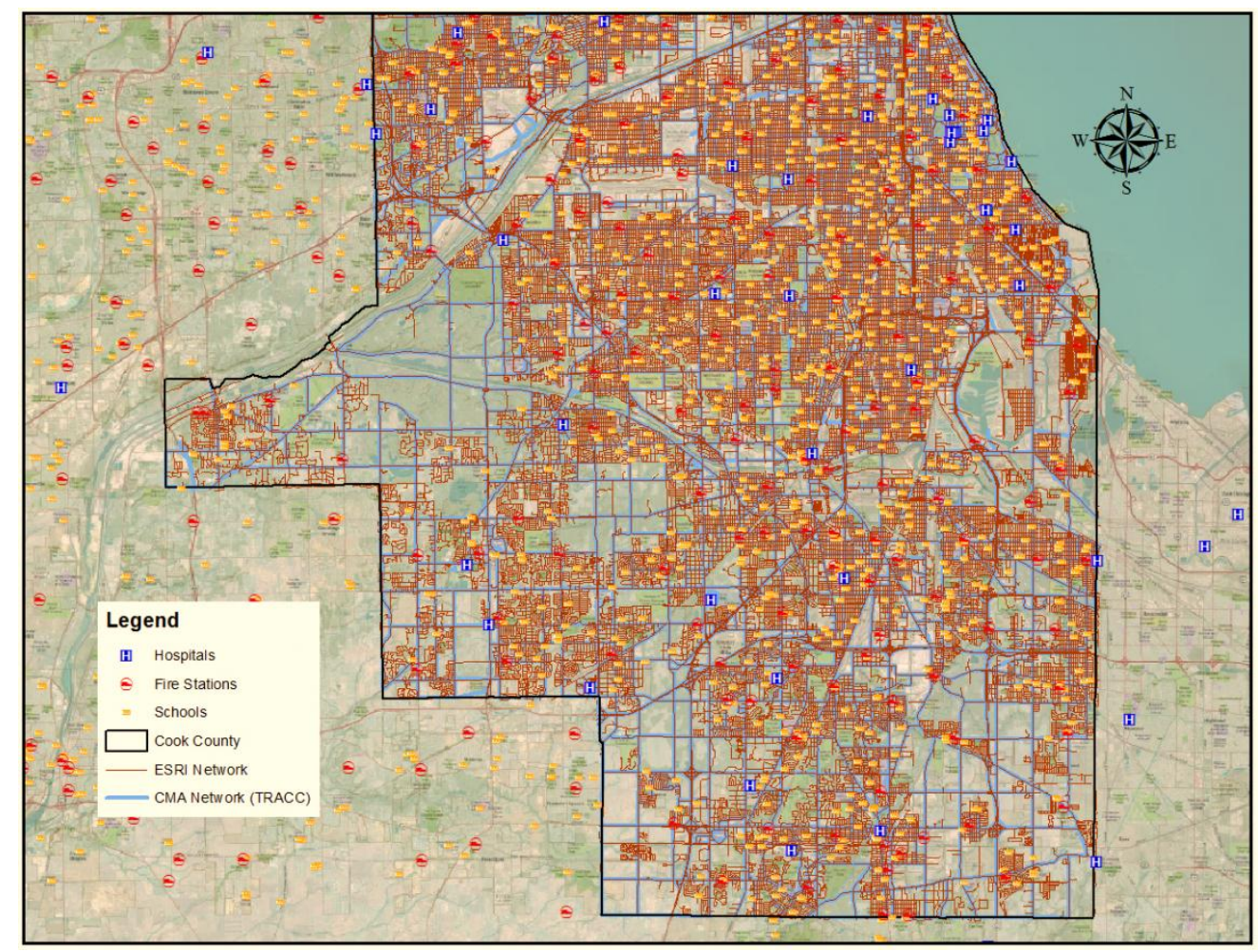

Figure 7.4: Southern Cook County 


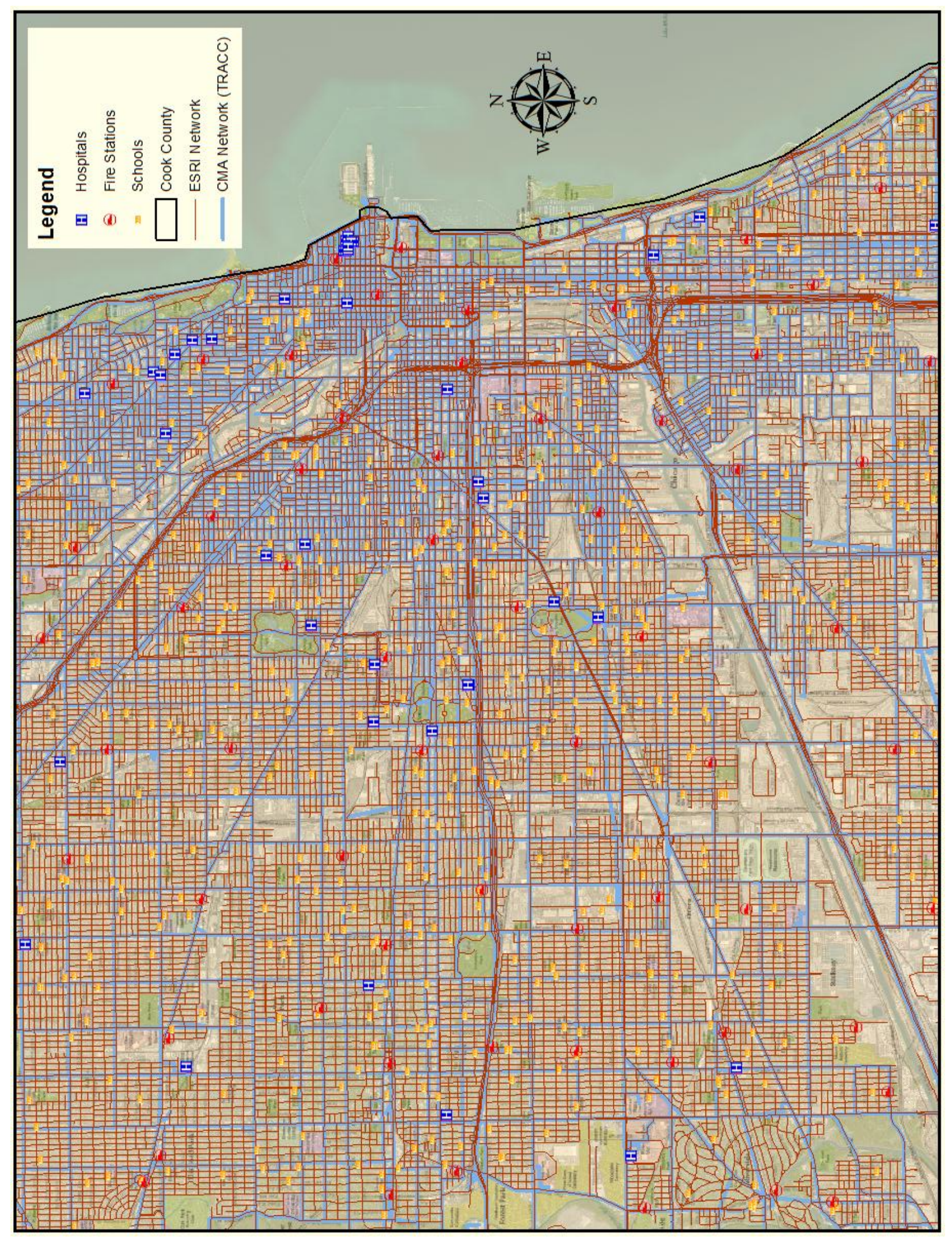

Figure 7.5: Chicago Business District and Surrounding Area 


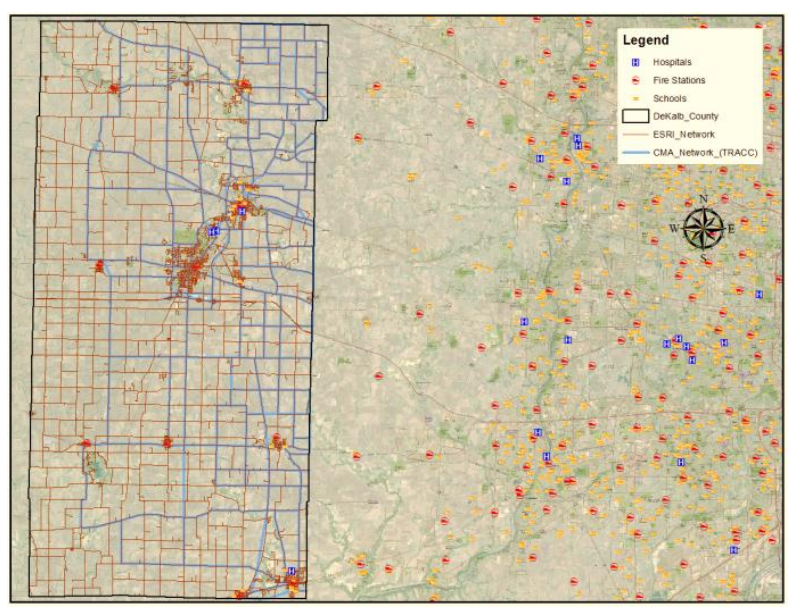

Figure 7.6: DeKalb County

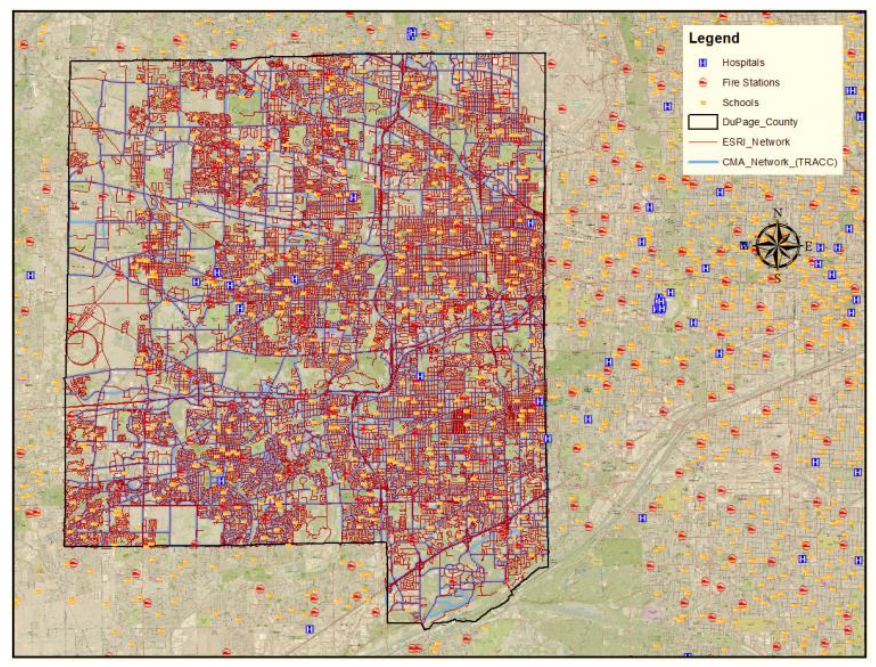

Figure 7.7: DuPage County

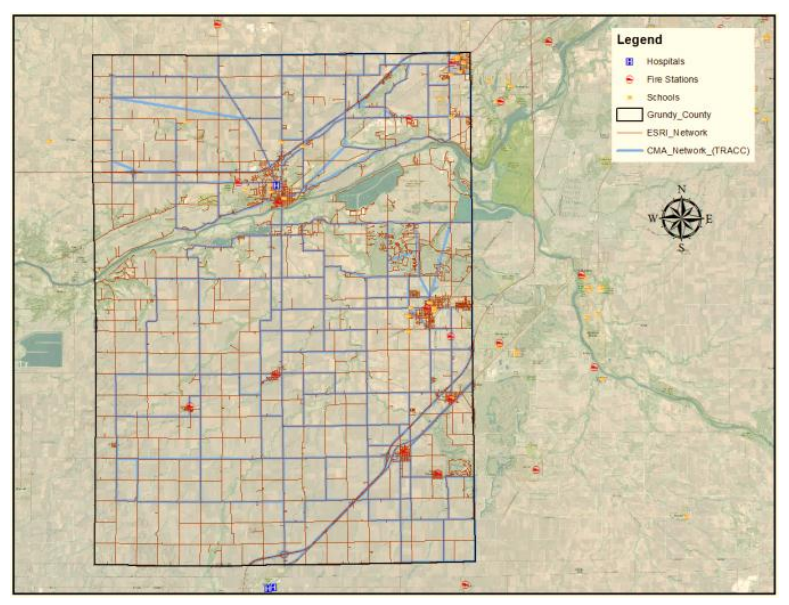

Figure 7.8: Grundy County 


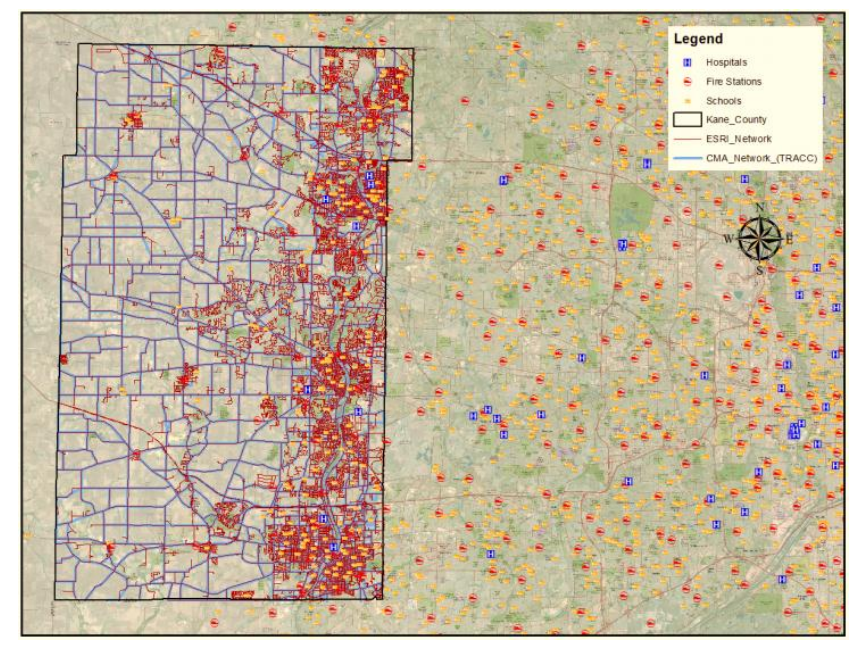

Figure 7.9: Kane County

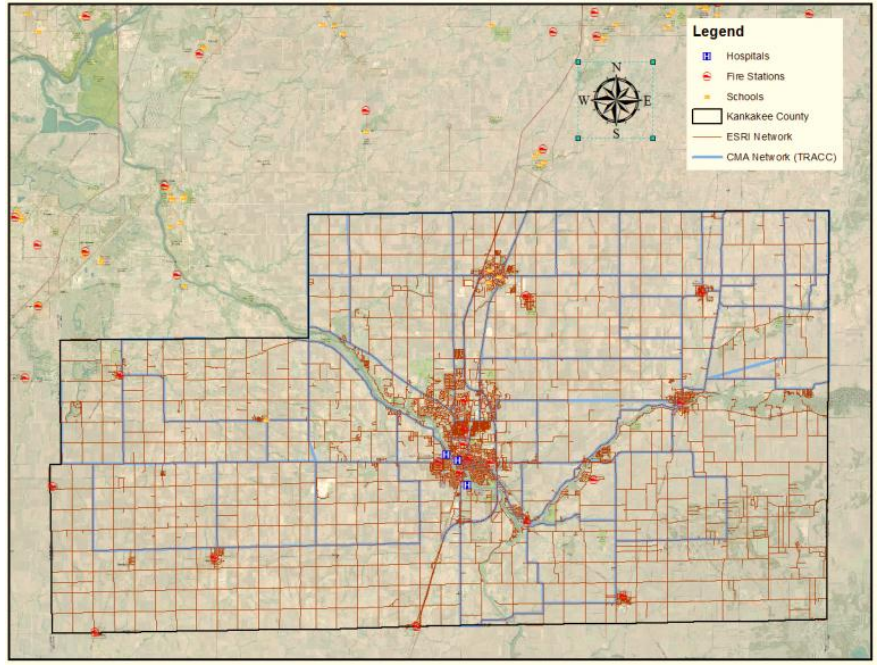

Figure 7.10: Kankakee County (based on CMAP and other regional data)

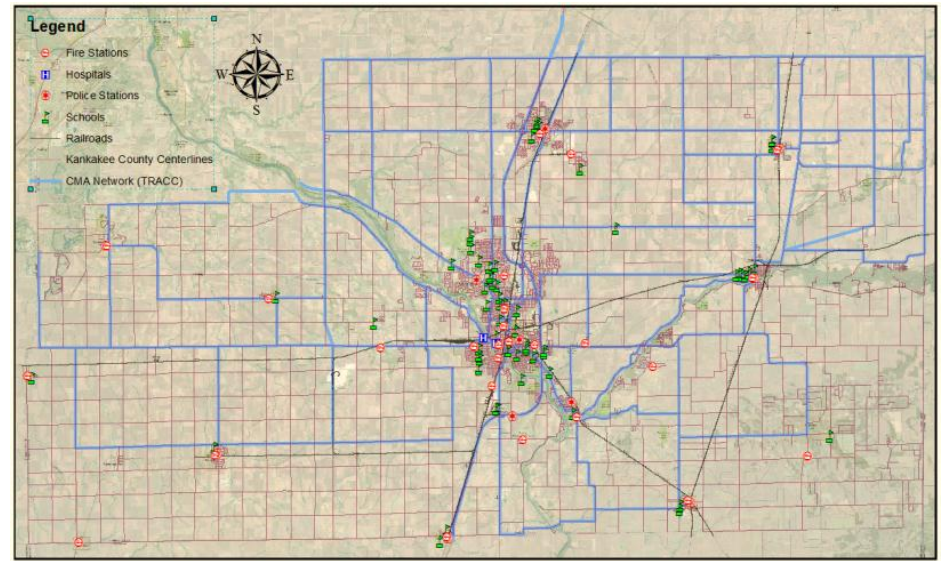

Figure 7.11: Kankakee County (detailed data layers have been made available) 


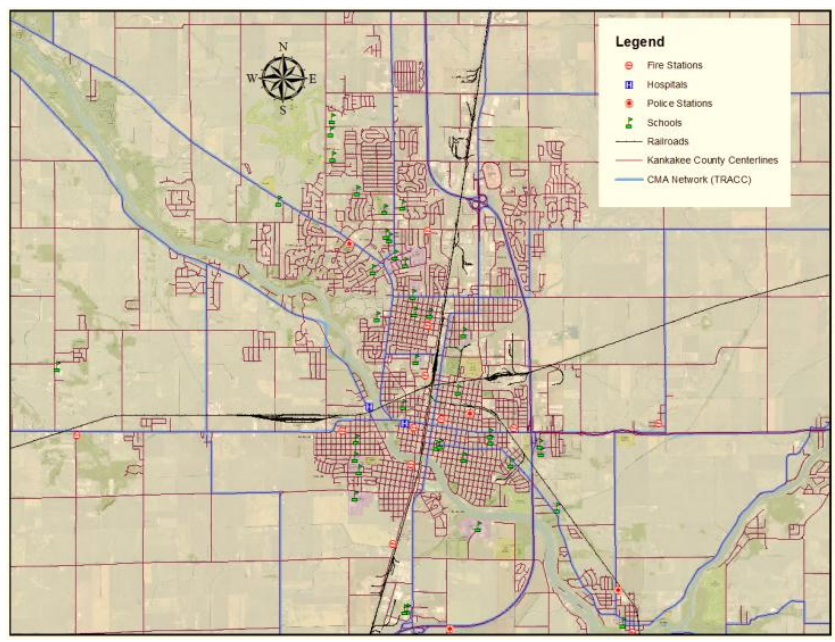

Figure 7.12: Kankakee (detailed data layers have been made available)

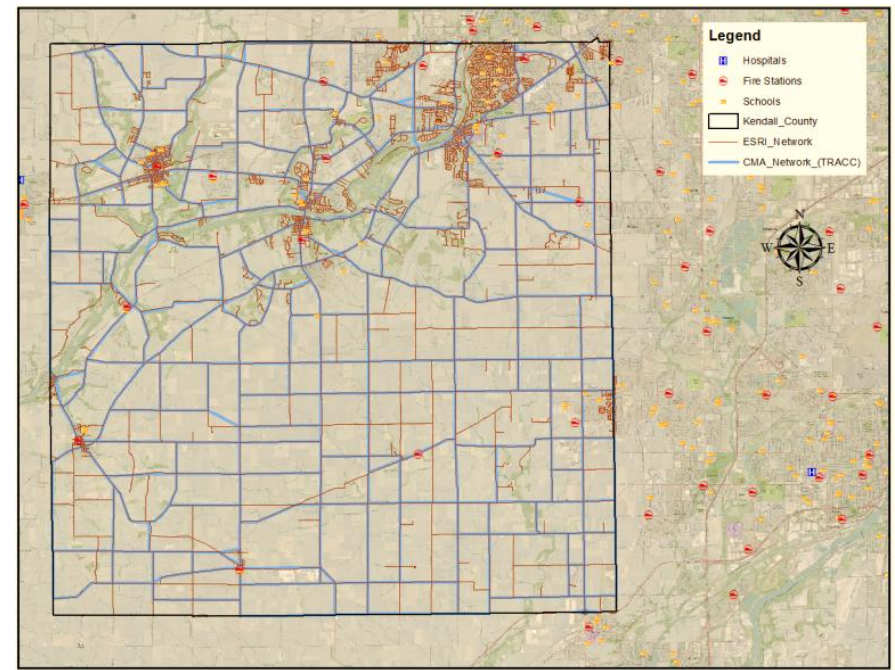

Figure 7.13: Kendall County

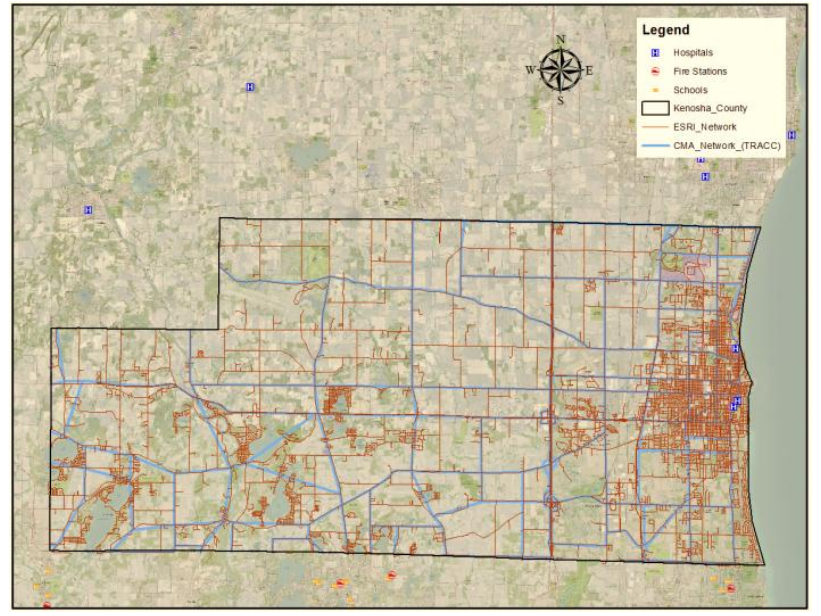

Figure 7.14: Kenosha County 


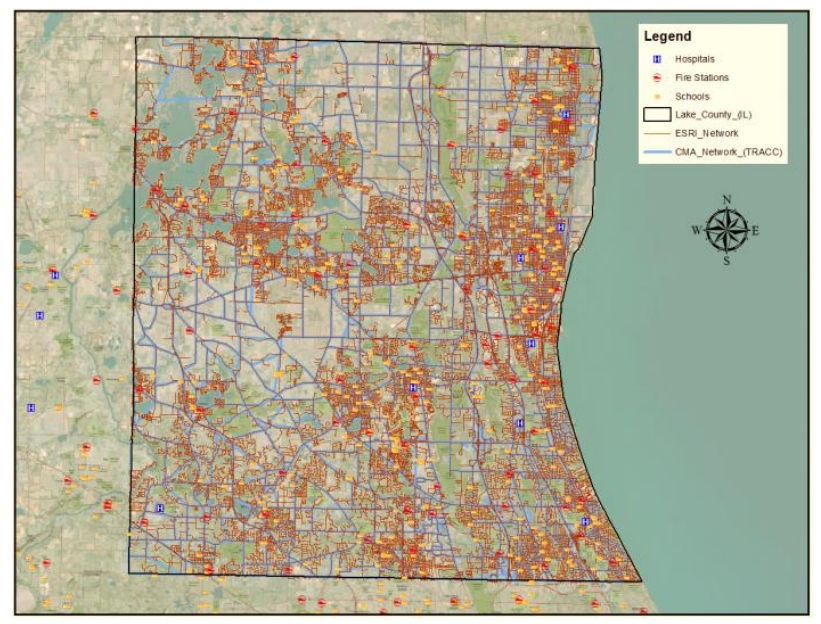

Figure 7.15: Lake County (Illinois)

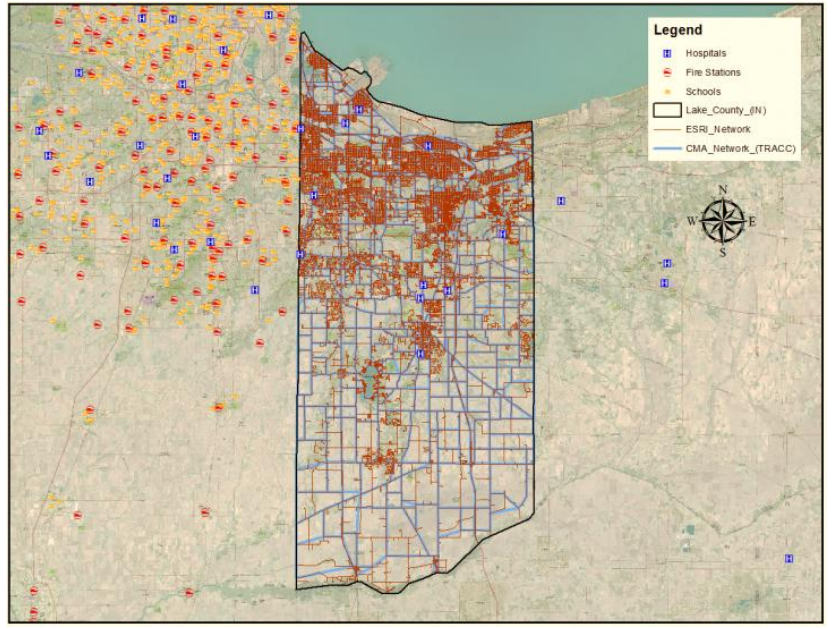

Figure 7.16: Lake County (Indiana)

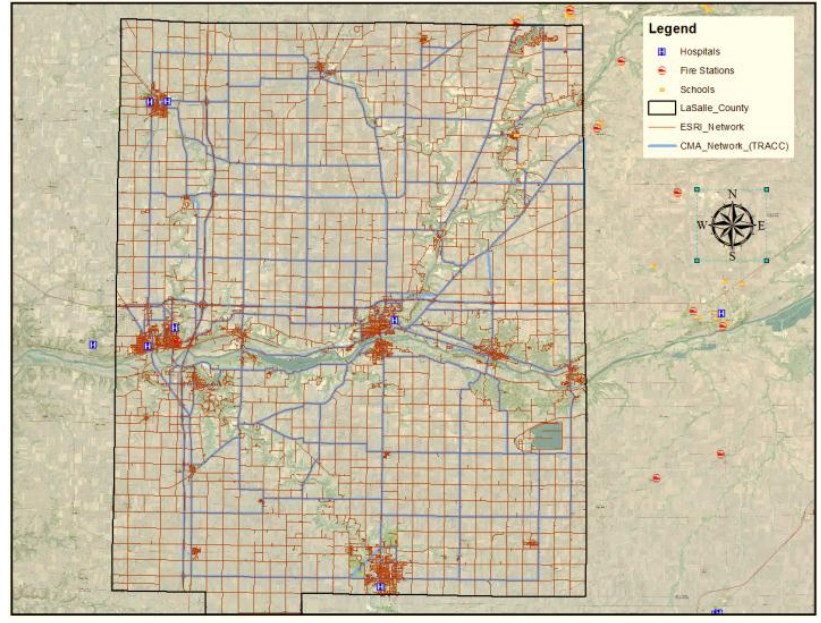

Figure 7.17: LaSalle County 


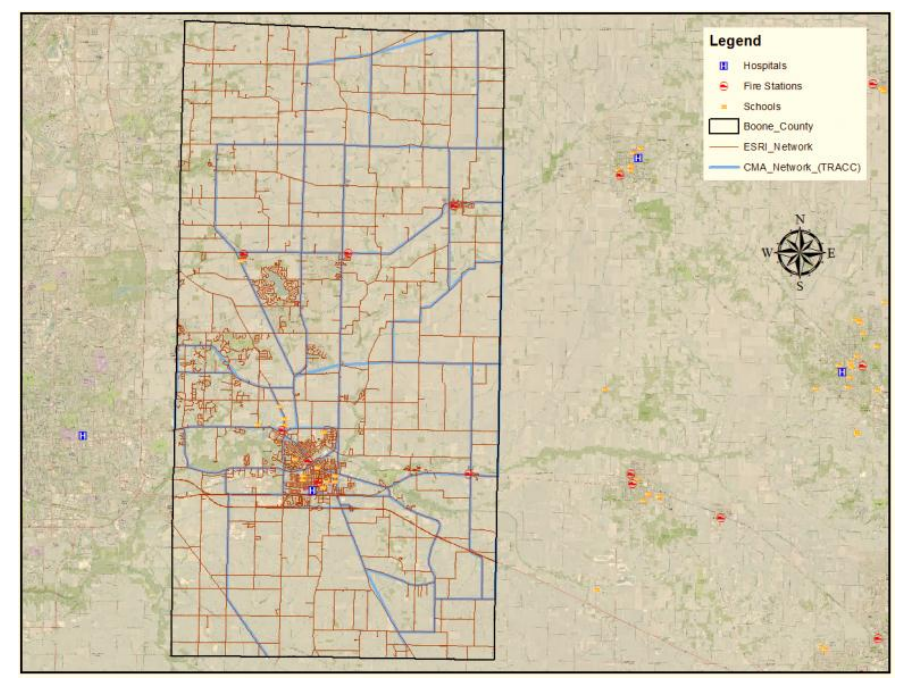

Figure 7.18: McHenry County

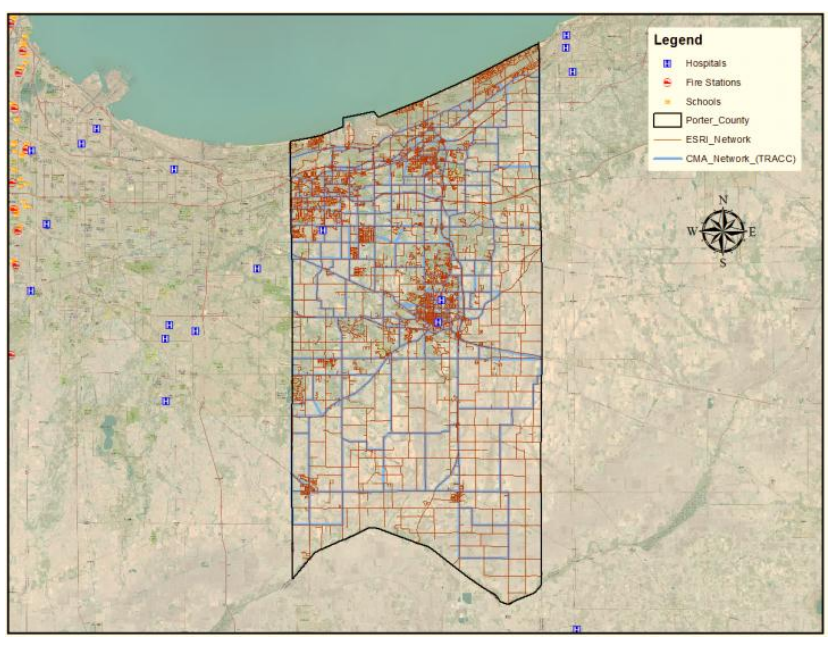

Figure 7.19: Porter County

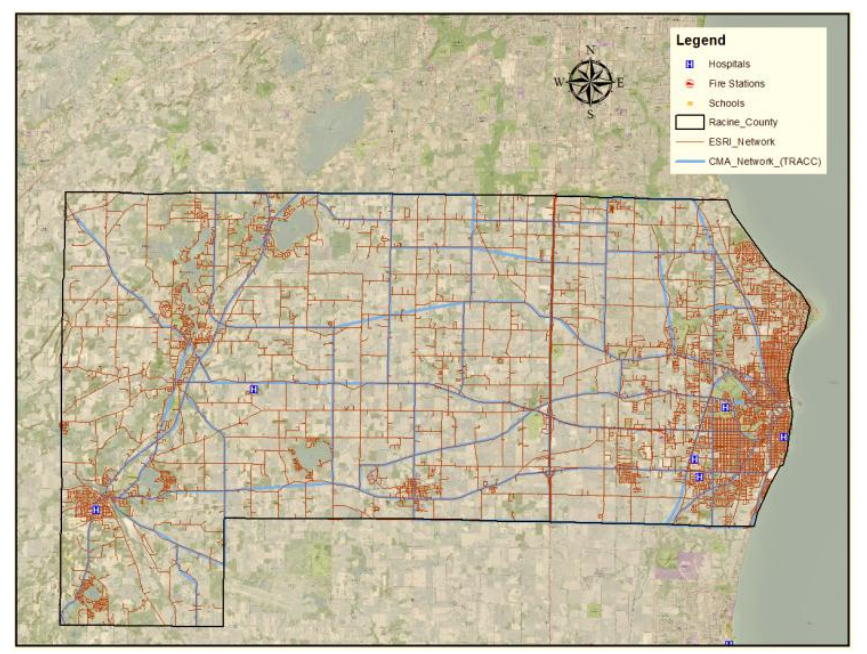

Figure 7.20: Racine County 


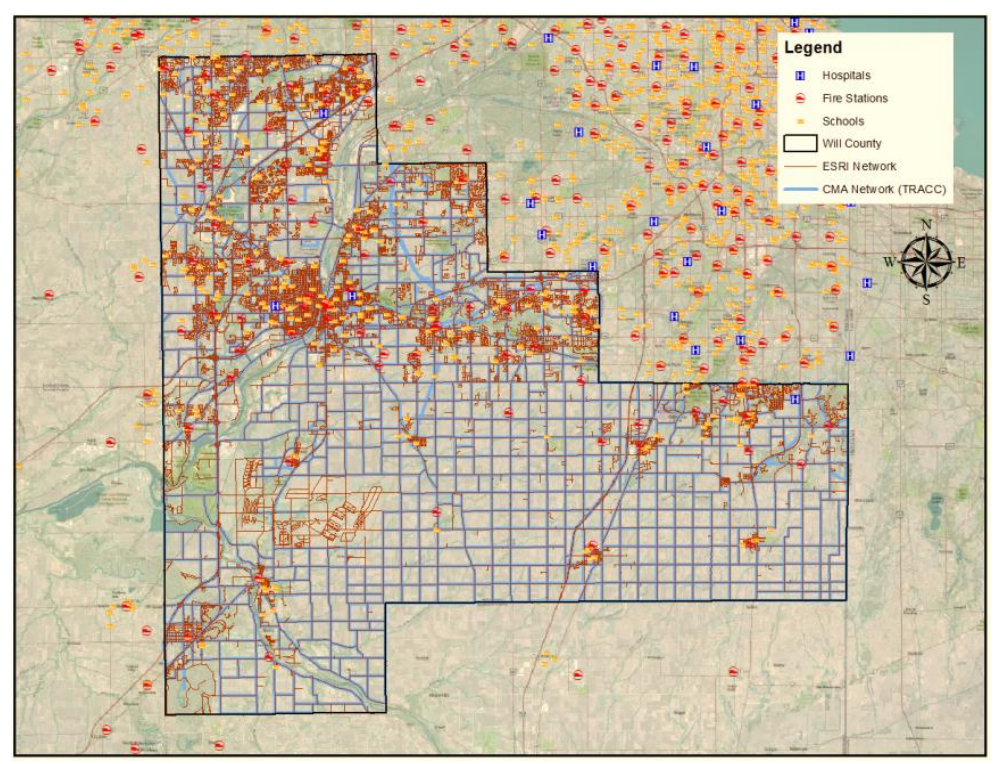

Figure 7.21: Will County 


\subsection{RTSTEP User's Manual}

The RTSTEP User's Manual describes the capabilities and operation of the RTSTEP software without going into details of the underlying TRANSIMS foundations. The graphical user interface allows for configuration of evacuation cases and visualization of the output from the corresponding TRANSIMS runs. The documentation is helpful to understand the scope of capabilities developed under this project.

\subsubsection{Introduction to RTSTEP}

\subsubsection{Introduction}

The RTSTEP software is distributed with 4 primary components. The first component is a software package containing all TRANSIMS executables which underlie the actual simulation of the evacuation. The second component is the tools EvacDemand and EvacNet, which are tools designed specifically for RTSTEP. The third component is the RTSTEP Evacuation Planner software used for case management and the RTSTEP Visualizer software used for both visual case configuration and displaying case results. The final component is a collection of commonly useful data sets which feed all other components.

Once the RTSTEP distribution has been installed, the software is ready for use. The RTSTEP Evacuation Planner software is a central hub for the overall software package. Open it by double click on the Evacuation Planner executable to begin configuring and running RTSTEP cases.

\subsubsection{RTSTEP Evacuation Planner}

This section describes the details of running the RTSTEP Evacuation Planner. The Evacuation Planner software is the main utility used to estimate and evaluate evacuation scenarios, and it is used to call the other executables developed for the RTSTEP project as well as the TRANSIMS executables used to estimate the evacuation travel demand. Following is a description of the process that must be done in order to take the TRANSIMS average day travel demand files and turn them into the demand files that contain evacuation information that can be visualized using RTSTEP Visualizer.

First the RTSTEP Evacuation Planner program must be launched. The RTSTEP Evacuation Planner will use the average day files plus a few special files (described in the RTSTEP Technical Documentation) to create a configuration file to be used by RTSTEP Visualizer. Once the configuration file has been created by the RTSTEP Evacuation Planner, the user can start up RTSTEP Visualizer using the Evacuation Planner in order to configure an evacuation scenario. The documentation shown in Section 3 below will show you how to navigate around the network, how to use the Time Control Bar, and how to use the Menu options. Of particular importance is the menu that allows you to configure evacuation data including adding evacuation areas, evacuation shelters, evacuation routes, and plumes.

Once the evacuation data is configured, the RTSTEP Evacuation Planner is used to run the TRANSIMS model using the evacuation scenario defined in RTSTEP Visualizer. From the Planner, Transims executables can be called and the network can be run through all of Transims steps including the Microsimulator to produce a new snapshot file that contains the evacuation data. RTSTEP Visualizer is then re-launched, and the network can now be visualized with evacuation data shown. 


\subsubsection{File Menu}

After launching the Evacuation Planning Tool users need to select one of the options for starting the scenario. In the File menu they can select the ability to either create a New Configuration File or Open an existing Configuration File. The modified Configuration File can be also saved from this menu. In the following example we will work from an existing Configuration File.

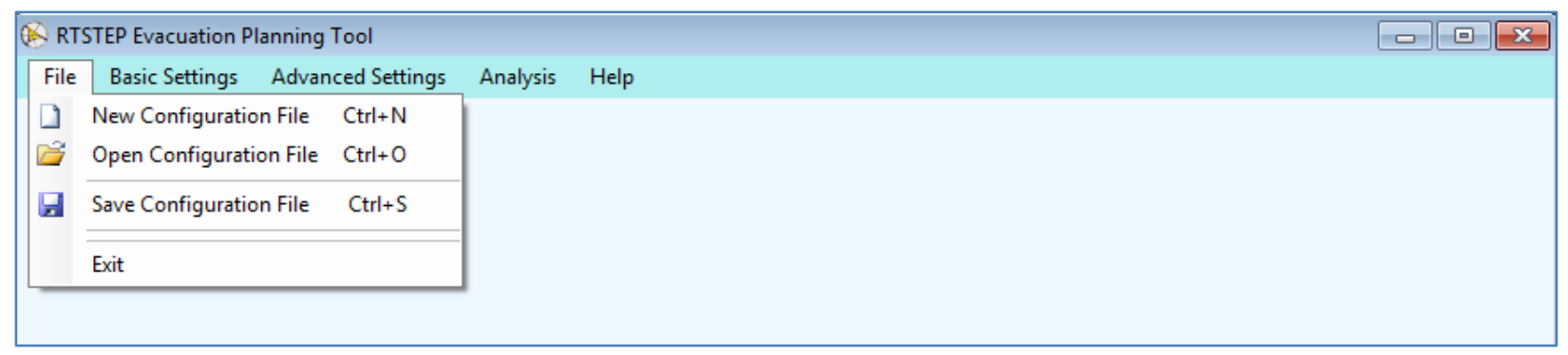

Figure 7.22. Content of the File Menu

Chose the Open Configuration File option and navigate to your_config_file.xml file. After loading the file, the Project Setup frame will be displayed (see Figure 7.23). The button Show/Hide Advanced Settings will provide an access to the setting files:

- Average Day Travel Demand and Link Delay Files

- Spatial Data Files

- Network Files

After modifying paths to the specific files, hit the OK button located at the bottom of the frame. The project setup will be finished. For further setup, proceed to the right of the top menu bar. 


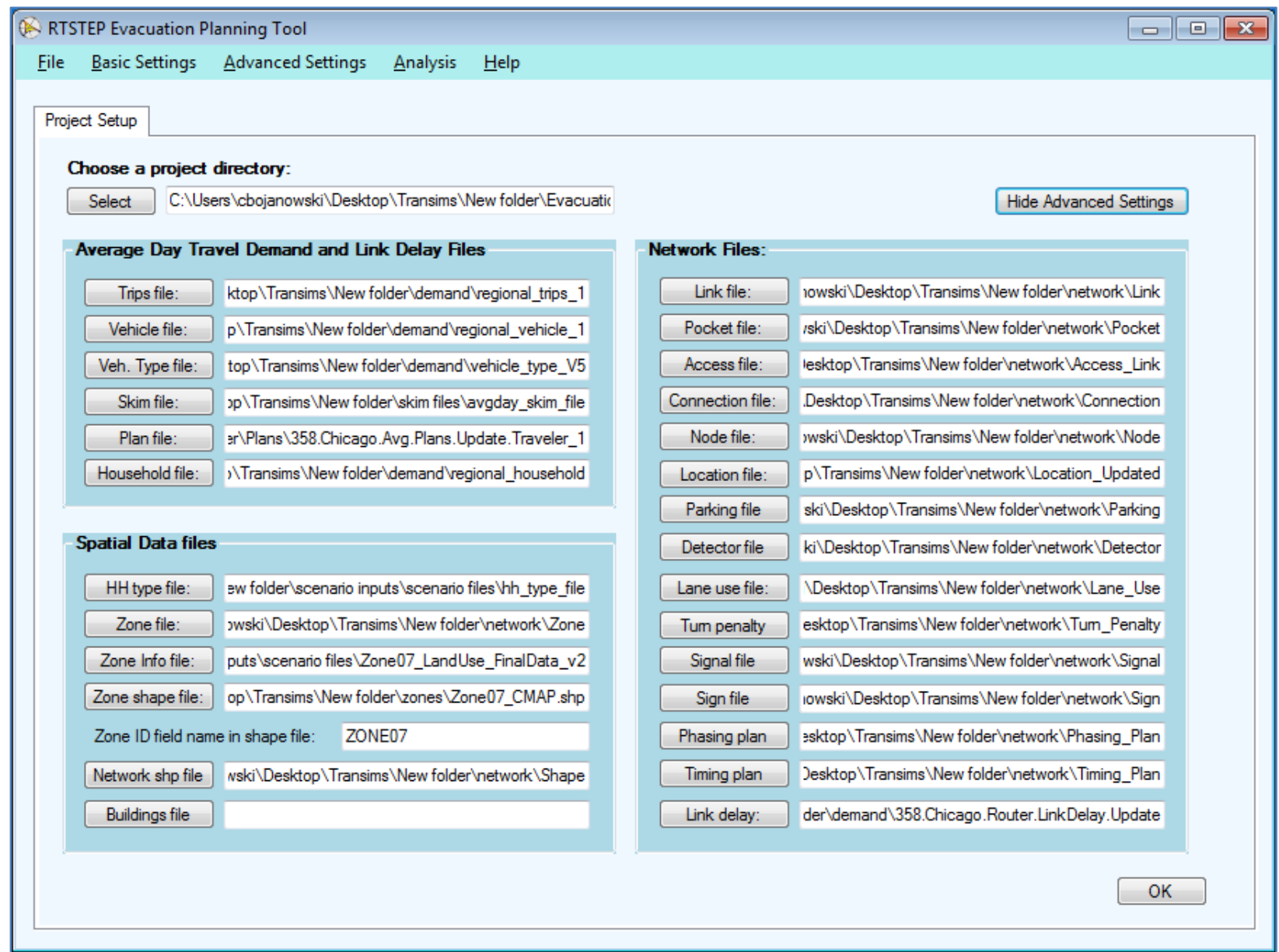

Figure 7.23. Project Setup panel

\subsubsection{Basic Settings Menu}

In the next step the Basic Settings menu should be launched. As shown in Figure 7.24, it provides access to the following settings:

- Percentage of total demand to simulate, which reduces the number of simulated vehicles in the evacuation scenario,

- Model Timeframe, which controlls the start and the end time of the evacuation process simulation.

The Intersection control mode in the Microsimulator can be also turned on here. The settings can be applied by hitting the OK button. 


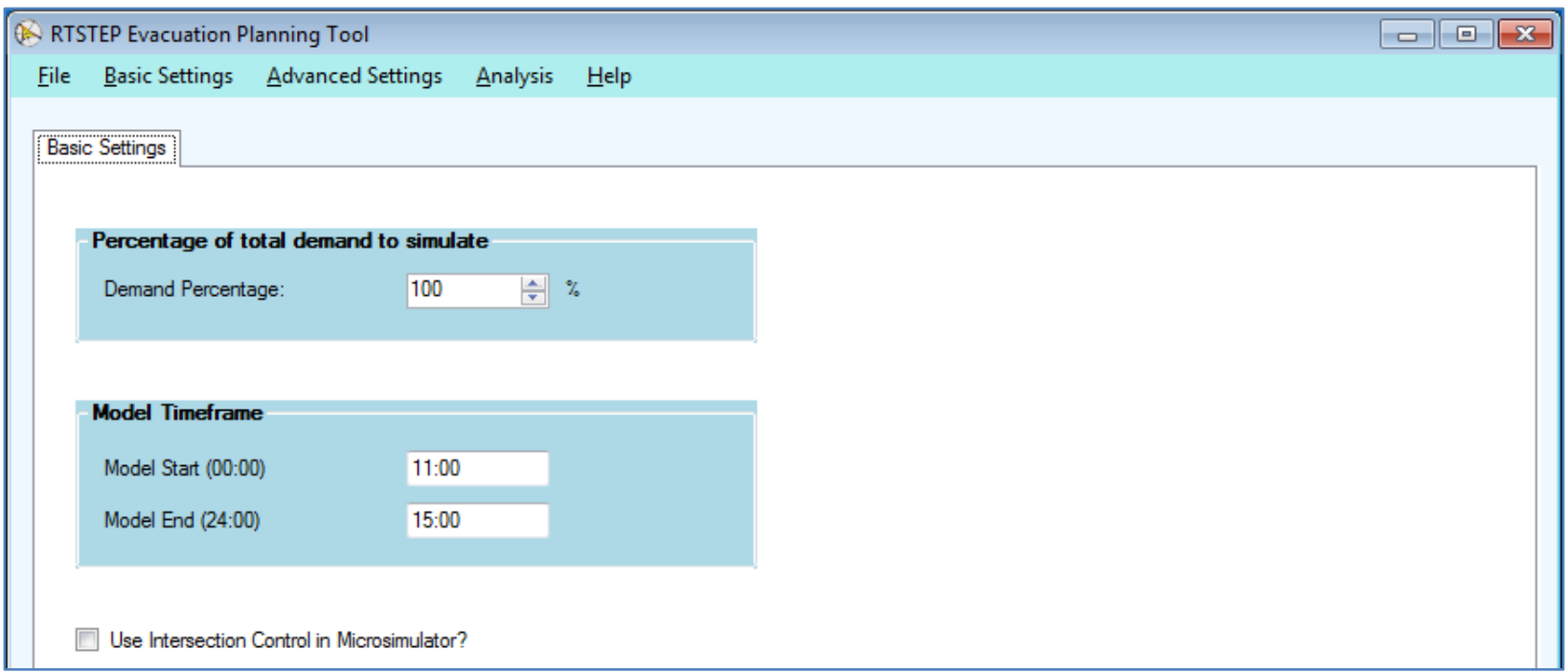

Figure 7.24. Basic Settings panel

\subsubsection{Advanced Settings Menu}

In the next step the Advanced Settings Menu should be accessed. Three groups of settings are available:

- Demand Settings,

- Transims Settings, and

Advanced Settings Analysis
Demand Settings
Transims Settings
Scenario Settings

- Scenario Settings.

\subsection{Demands Settings}

This section of the program allows the parameters for the evacuation demand model to be edited by the user. This setting is currently disabled in the Evacuation Planner software. For users who want to modify the demand model parameters to values other than the default values, the EvacDemand.ctl control file can be edited directly. See the EvacDemand Technical report for details.

\subsection{Transims Settings}

The Transims Settings menu is shown in Figure 7.25. In this menu, basic settings for the Transims Router, Simulator and RTSTEP Visualizer can be set. Router Settings consist of:

- Link-delay update rate in trips per update, and

- Percent of random independence.

The Simulator Settings menu allows user modification of:

- Maximum wait time in seconds, and

- Permission probability percentage.

RTSTEP Visualizer settings allow for modifications of offset and scaling factors for: 
- ZONE File Geographic Projection, and

- NETWORK Files Geographic Projection

Also in the RTSTEP Visualizer section, the Satellite Theme can be adjusted by switching between the gray and color modes.

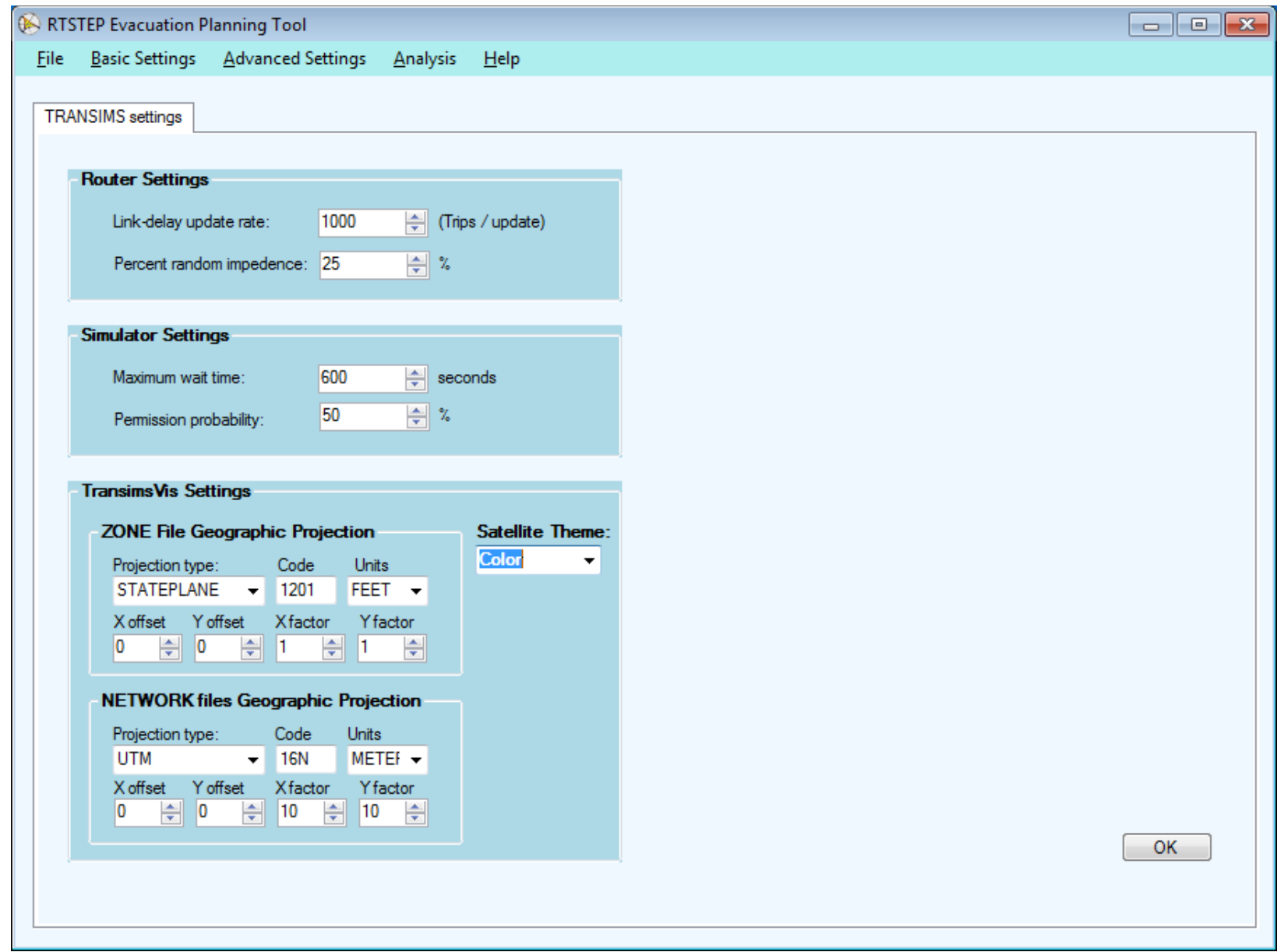

Figure 7.25. Transims Settings in the Advanced Settings menu

\subsection{Scenario Settings}

Once the Scenario settings are selected from the Advanced Settings menu, the Define Scenario frame pops up with two sub menus (see Figure 7.26). In the first setting on the top, the scenario definition input files option needs to be specified. The file list includes:

- Routes File,

- Plume File,

- Evacuation Zone File,

- Response File, 
- Shelter File, and

- Evacuation Vis File.

If a new case is considered, then the Evac Zone, Response and the Shelter Files must be left blank.

The Evac Demand Settings frame includes:

- Home-based trip purpose codes,

- Number of housejold types in HH Type File,

- Average Vehicle Occupancy for Evacuation Households,

- Allowable Reduction in Utility for Destination Choice,

- Minimum allowable ratio of origin to evacuation travel time, and

- Trip scaling factor used in generation.

After all the necessary fields are defined, click the OK button on the bottom of the window.

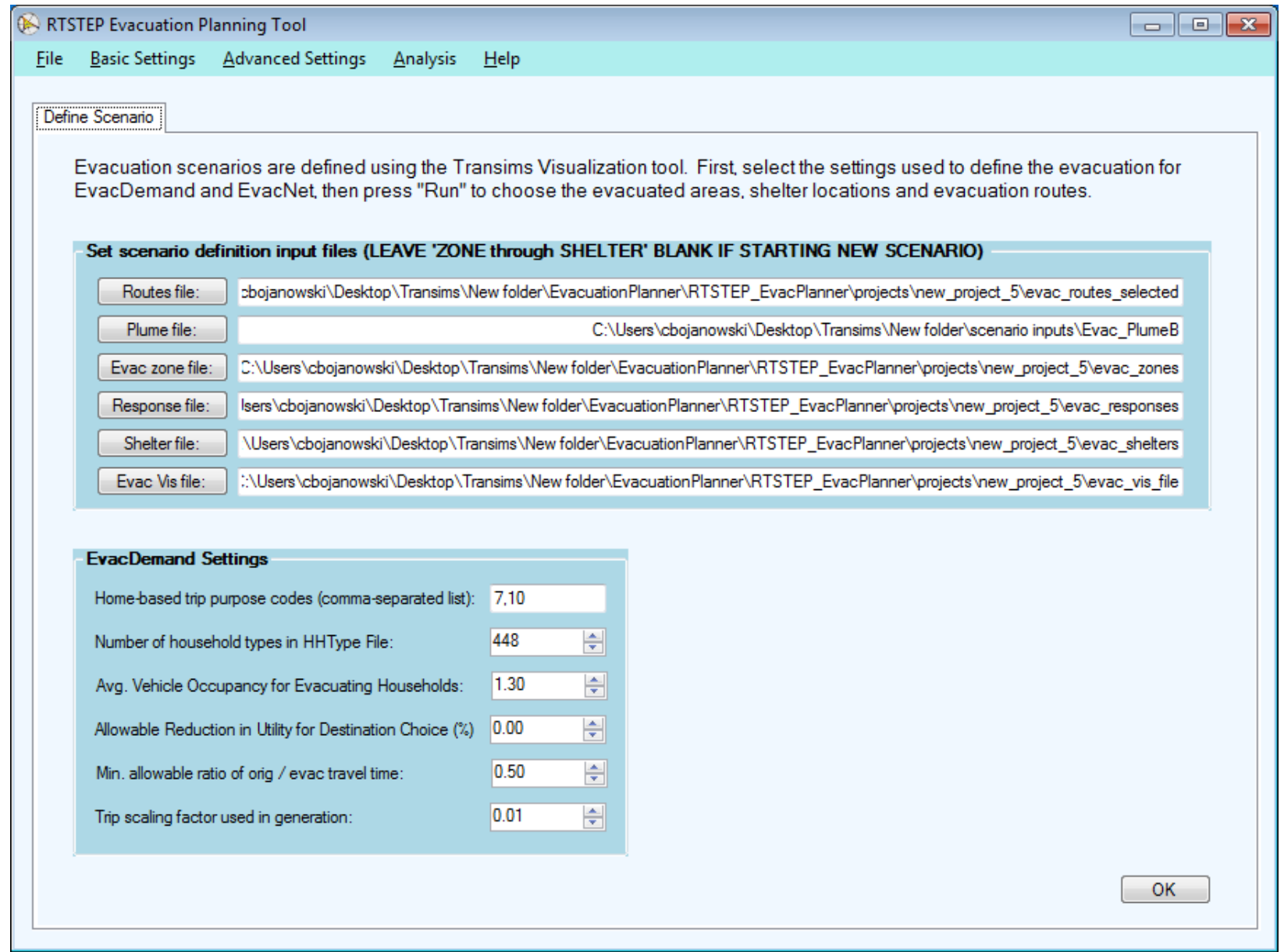

Figure 7.26. Scenario Settings in the Advanced Settings menu 


\subsubsection{Analysis Menu}

The Analysis Menu contains the following options (as shown in Figure 7.27):

- Create/Edit Scenario

- Run Model

- Run Model - Evacuees only

- Run Submodel

- View Results

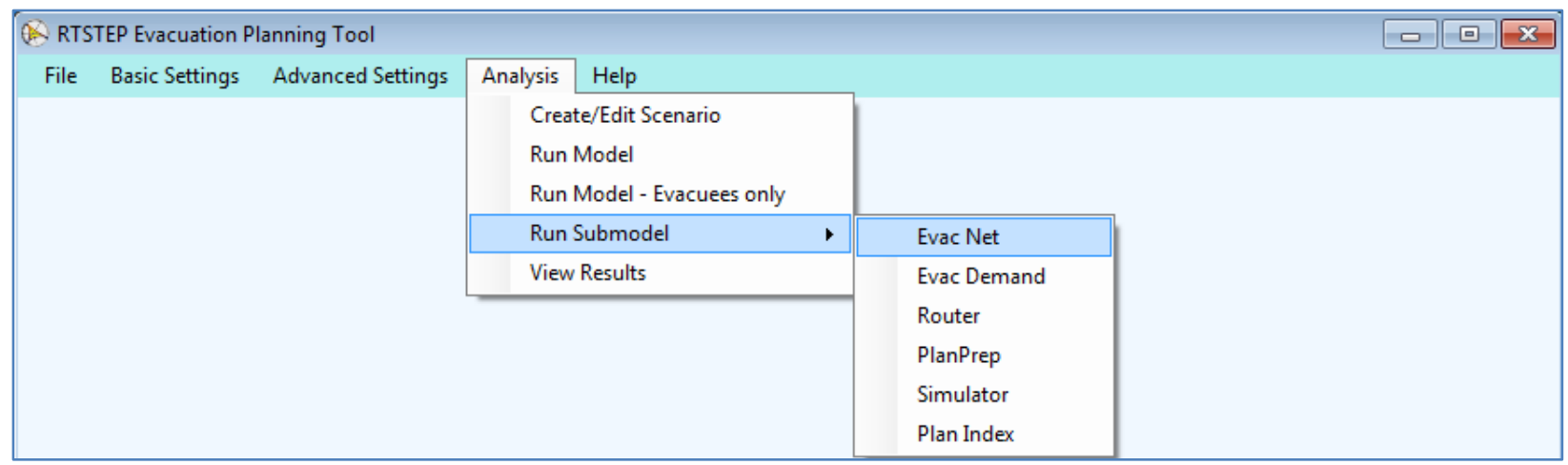

Figure 7.27. Content of the Analysis Menu

Once the scenario settings with all the input file paths are defined, the scenario itself can be modified within the RSTEP Evacuation Configuration Tool. This application is accessed from the Analysis menu under the option Create/Edit Scenario. The RTSTEP Evacuation Configuration Tool is described in detail in Section 7.5.3 of this manual. After editing the scenario, the entire evacuation model can be run by selecting the Run Model option. If the user is interested in running the case considering only the vehicles in the affected areas for evacuation, then the Run Model - Evacuees only option should be selected. Step by step Transims analysis can be performed by selecting solvers from the Run Submodel option:

- $\quad$ Evac Net

- Evac Demand

- Router

- PlanPrep

- Simulatore, and

- Plan Index

If the option Run Model (or Run Model - Evacuees only) is chosen, the Results are automatically loaded into RTSTEP Visualizer and can be visualized. If you have previously run the model, the results will have already been stored. You can then select the View Results option, and the previously run results will be loaded into RTSTEP Visualizer and can be visualized. 


\subsubsection{RTSTEP Evacuation Configuration Tool}

After the model has been run using the RTSTEP Evacuation Planner and is loaded into RTSTEP Visualizer as described in the previous section, it can be configured using the RTSTEP Evacuation Configuration tool and visualized. Once the Evacuation Menu has been selected, you should see the Options for the Evacuation Menu in the Map Options Frame on the left side of the display, the corresponding Map View in the middle, and the Attribute Menu on the right as shown in Figure 7.28. At this state, the Visualizer provides functions for defining, editing, and viewing an evacuation scenario by using the Evacuation Menu, Map View, and Attribute Menus. The Evacuation Menu includes functions for the evacuation scenario. The Map View shows evacuation elements, and the Attribute Menu shows parameters of evacuation elements for editing.

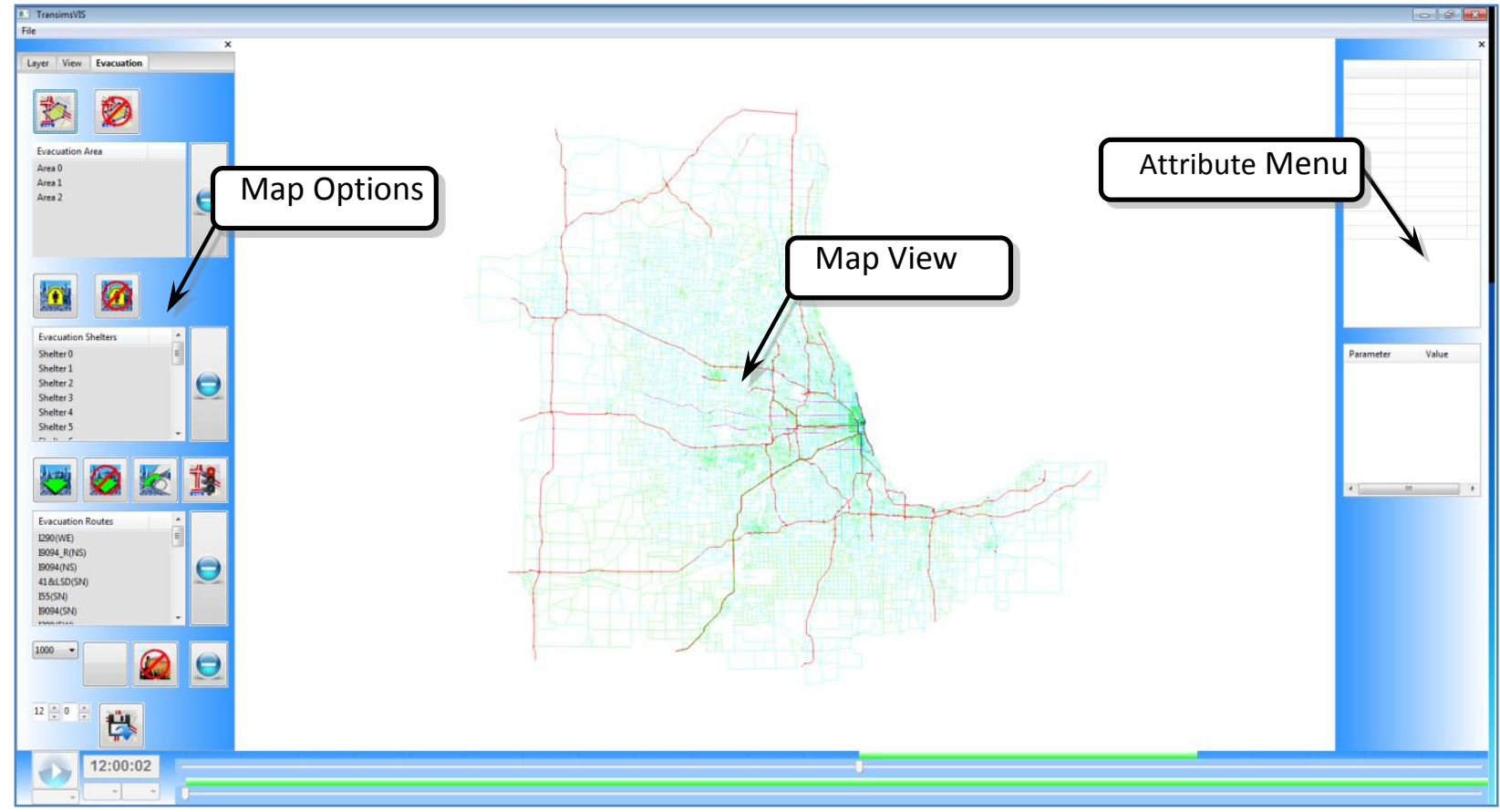

Figure 7.28. Visualizer in Evacuation Mode 


\subsubsection{Visualization mode}

The evacuation menu includes two visualization modes: the configuration mode and the evacuation mode. The visualization mode is defined by the VISULIZER_MODE key in the RTSTEP_Visualizer.ctl control file as follows:

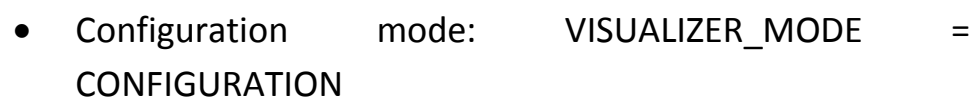

- Evacuation mode: VISUALIZER_MODE =EVACUATION

In the configuration mode, the evacuation menu is active. Users can create a new evacuation scenario with elements of the evacuation area, evacuation shelters, evacuation routes, dispersion area, and the evacuation start time. Users can also edit these evacuation related elements using the Evacuation Menu, Map View, and Attribute Menu. In the evacuation mode, the evacuation menu is inactive. Users can only visualize a pre-defined evacuation scenario.

\subsubsection{Functions of the Evacuation Menu}

The evacuation menu includes five functions as follows:

1. Evacuation area

2. Evacuation shelters

3. Evacuation routes

4. Dispersion area

5. Evacuation start time and scenario saving

For the first four functions, two buttons, the Plus Button and the Minus Button, are designed with similar purposes. When the Plus Button is clicked, it will enable the corresponding evacuation element, which will be shown in the Map View; similarly, when the Minus Button is clicked, the corresponding evacuation element will be disabled and will disappear from the Map View.

\subsection{Evacuation Area}

The Evacuation Area function is used to define a set of evacuation areas with different levels of risk. The Evacuation Area function consists of three buttons and a list view. The Adding Evacuation Area Button is used to add a new evacuation area. The Deleting Evacuation Area Button is used to delete an existing evacuation area. The Evacuation Area List View lists the existing defined evacuation areas.
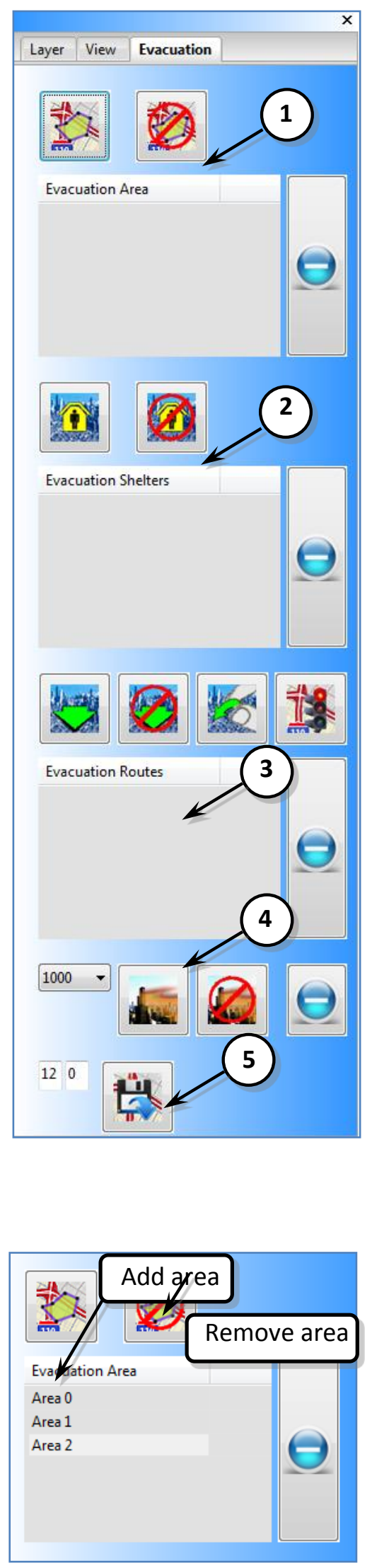
When the Adding Evacuation Area Button is clicked, users can use the mouse to create a new evacuation area in the Map View by defining a polygon. The newly created evacuation area will then be automatically added to the Evacuation Area List. Users can also define corresponding parameters for the newly created evacuation area using the Evacuation Area Parameter List View on the Attribute Menu. There are six parameters that can be modified as follows.

- Name

- Evac Pct: Evacuation percentage

\begin{tabular}{|ll|}
\hline Parameter & Value \\
\hline Name & Area 2 \\
Evac Pct & 100 \\
Response Median & 15 \\
Response Shape & 1.25 \\
Severity (1-5) & 3 \\
Population & 0 \\
& \\
& \\
\hline
\end{tabular}

- Response Median

- Response Shape

- Severity (1-5)

- Population

Users can double-click the Value field for each parameter and edit the value. An example of an evacuation area scenario with three risk levels is shown in Figure 7.29.

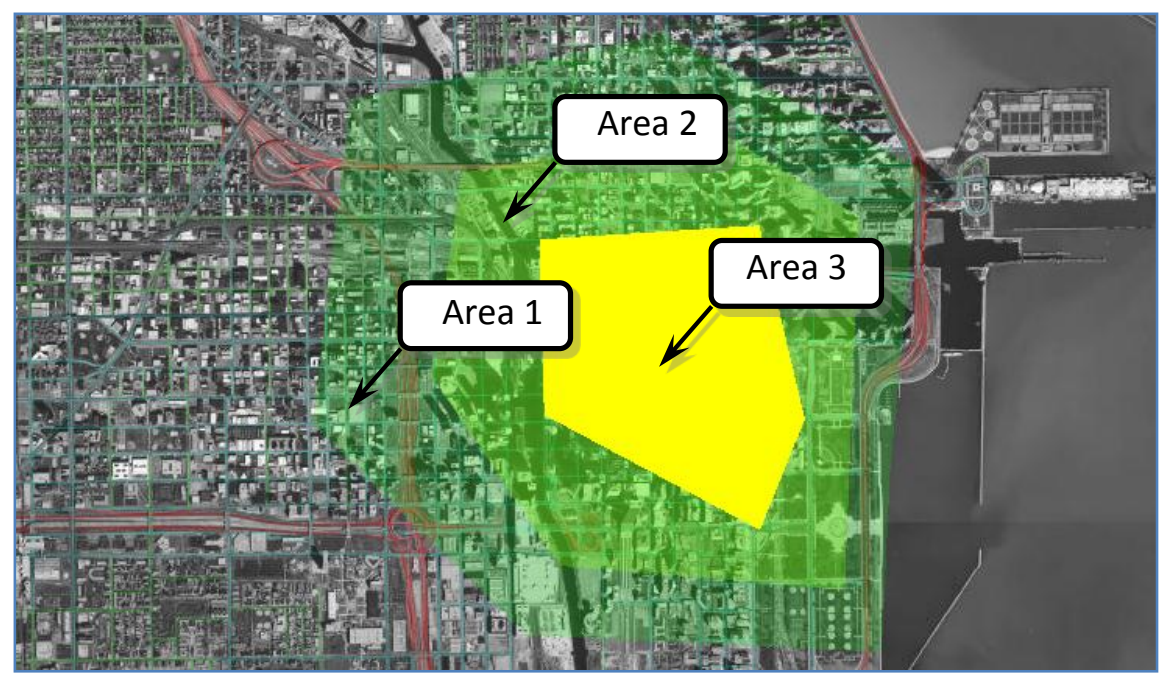




\subsection{Evacuation Shelters}

The Evacuation Shelters function is used to define a set of evacuation shelters in the map. The Evacuation Shelters function consists of three buttons and a list view.

The Adding Evacuation Shelter Button is used to add a new evacuation shelter. The Deleting Evacuation Shelter Button is used to delete an existing evacuation shelter. The Evacuation Shelters List View lists the existing defined evacuation shelters.

When the Adding Evacuation Shelter Button is clicked, users can use the mouse to create a new evacuation shelter in the Map View by clicking in the map with a shelter icon. The newly created evacuation shelter will then be automatically added to the Evacuation Shelter List.

Users can also define corresponding parameters for the newly created evacuation shelter using the Evacuation Shelter Parameter List View on the Attribute Menu.

There are three parameters that can be modified as follows.

- Name

- Capacity

- Desirability

Users can double-click the Value field for each parameter and edit the \begin{tabular}{|ll|}
\hline Parameter & Value \\
\hline Name & Shelter 0 \\
Capacity & 1000 \\
Desirability & 1 \\
& \\
& \\
\hline & \\
\hline
\end{tabular} value. An example of an evacuation shelters scenario is shown in Figure 7.30 . 


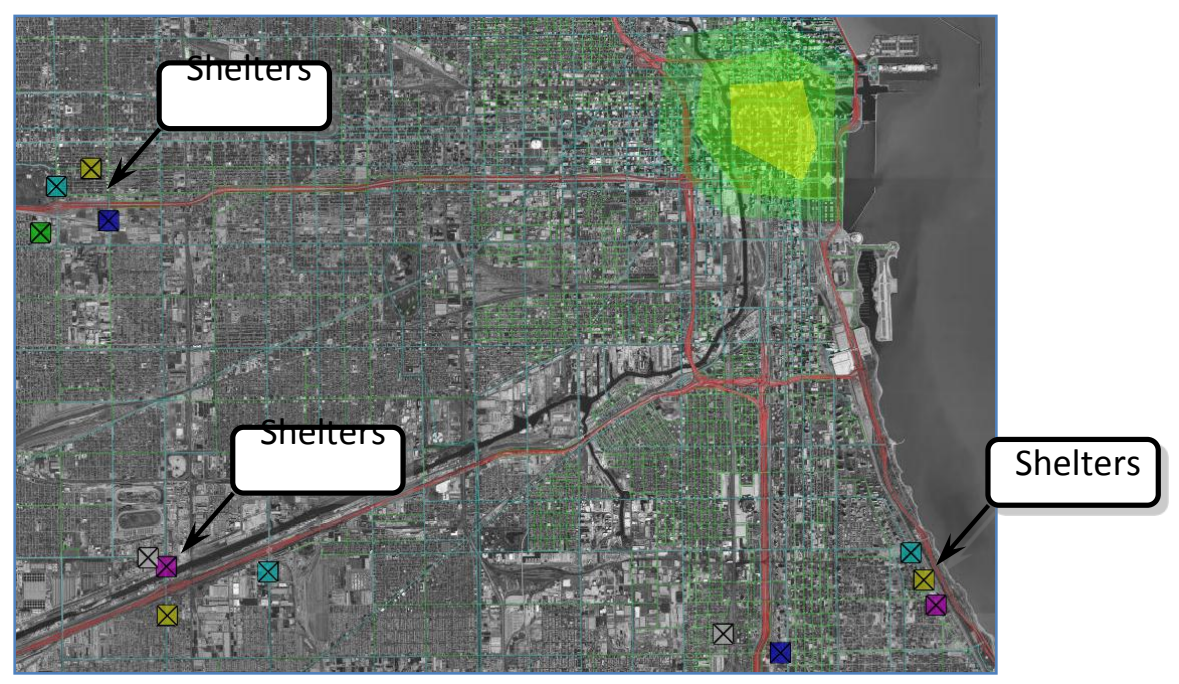

Figure 7.30. Evacuation Shelters Scenario

\subsection{Evacuation Routes}

The Evacuation Routes function is used to define a set of evacuation routes in the map. The Evacuation Routes function consists of five buttons and a list view.

The Adding Evacuation Route Button is used to add a new evacuation route. The Deleting Evacuation Route Button is used to delete an existing evacuation route. The Editing Evacuation Route Button is used to edit an existing evacuation route. The Editing Critical Intersection Button is used to add/delete a critical intersection along

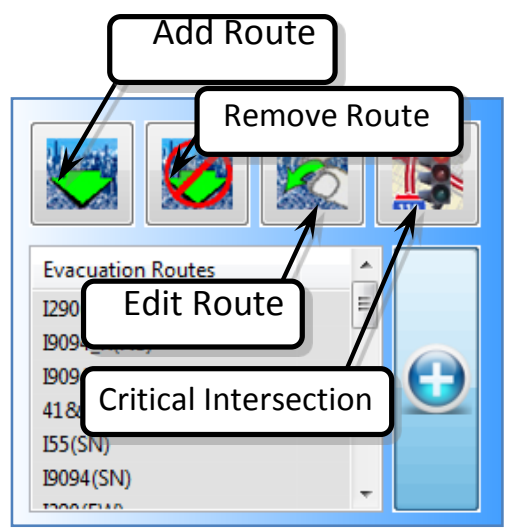
an existing evacuation route. The Evacuation Routes List View lists the existing defined evacuation routes.

When the Adding Evacuation Route Button is clicked, all the candidate evacuation routes will be shown in the Map View, and users can use the mouse to select an evacuation route by clicking in the evacuation route (selected evacuation routes will be highlighted as white in this case), which is shown in Figure 7.31. 


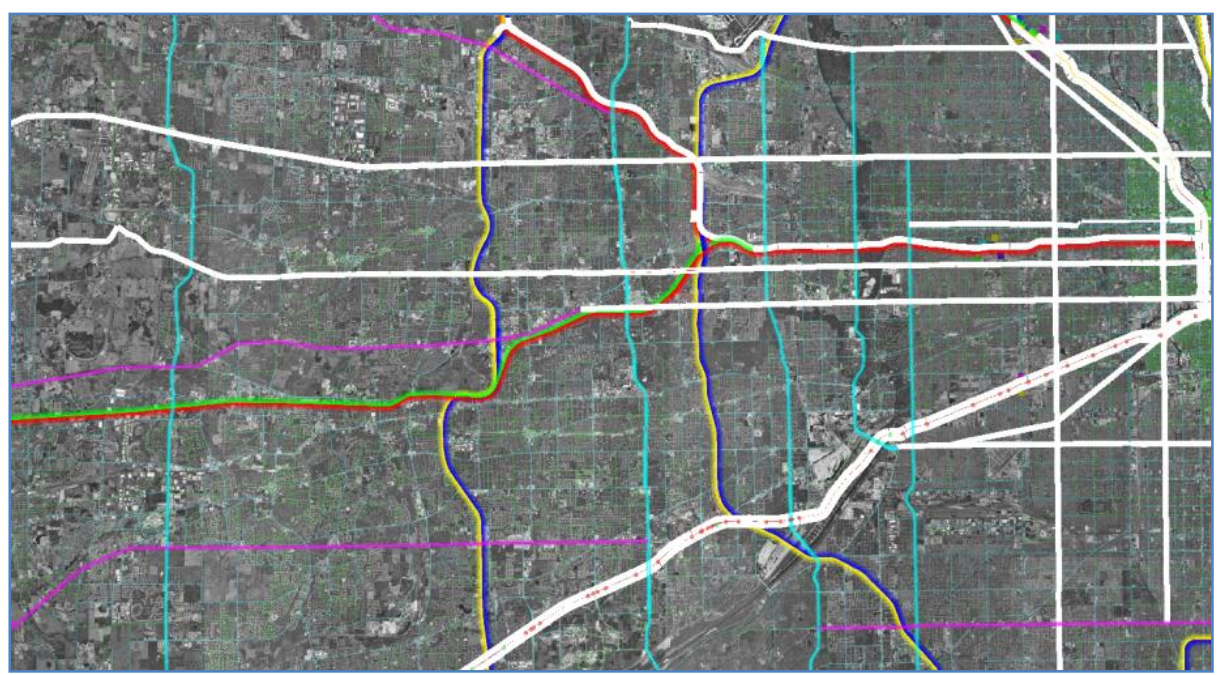

Figure 7.31. Adding Evacuation Route Mode

The newly created evacuation routes will then be automatically added to the Evacuation Routes List. Users can also define corresponding attributes for the newly selected evacuation route using the mouse.

By clicking the Editing Evacuation Route Button, users can trim the selected evacuation route as is shown in

Figure 7.32 (left). Colored links are used to distinguish different links along the evacuation route. By clicking the Editing Critical Intersection Button, users can add/delete a critical intersection along the selected evacuation route as is shown in

Figure 7.32 (right). The green nodes are critical intersections and the red nodes are managed intersections. An example of evacuation route scenario is shown in Figure 7.33.

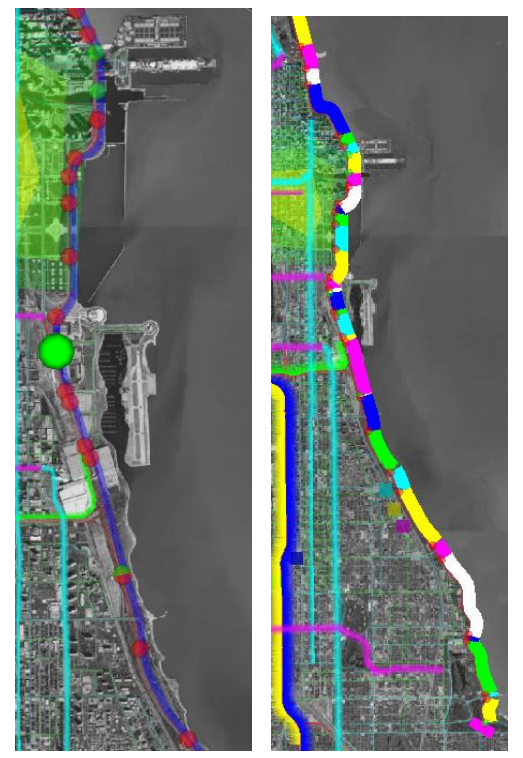

Figure 7.32. Adding Evacuation Route Mode 


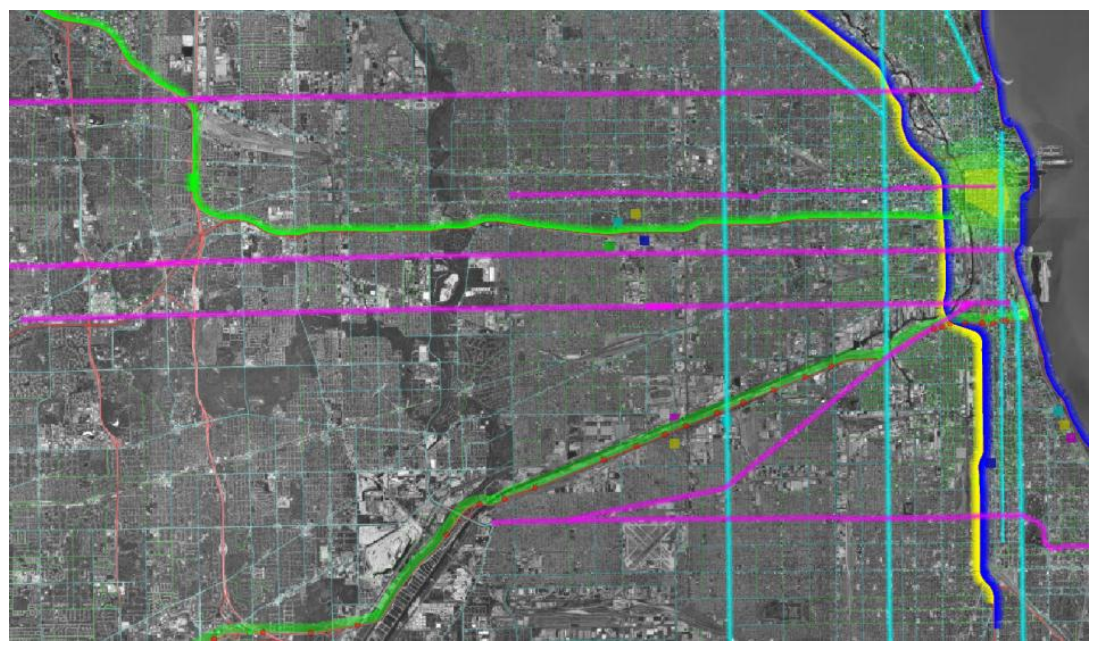

Figure 7.33. Evacuation Routes Scenario

\subsection{Dispersion Area}

The Dispersion Area function is used to define a dispersion area in the map. The Dispersion Area function consists of three buttons and a drop down list. The Adding Dispersion Area Button is used to create a dispersion area in the map. The Deleting Dispersion Area Button is used to delete an existing dispersion area in the map. The Dispersion Area Size Drop Down List is used to define the size of a dispersion area. Before creating a dispersion area, users need to select the size of the dispersion area using the Dispersion Area Size Drop Down List where two default values are provided: 1000 and 2500. When the user clicks the Adding Dispersion Area Button, the mouse cursor will turn to a red dot. After that, users click inside a pre-defined evacuation area and draw a dispersion area. See an example of dispersion area in Figure 7.34.

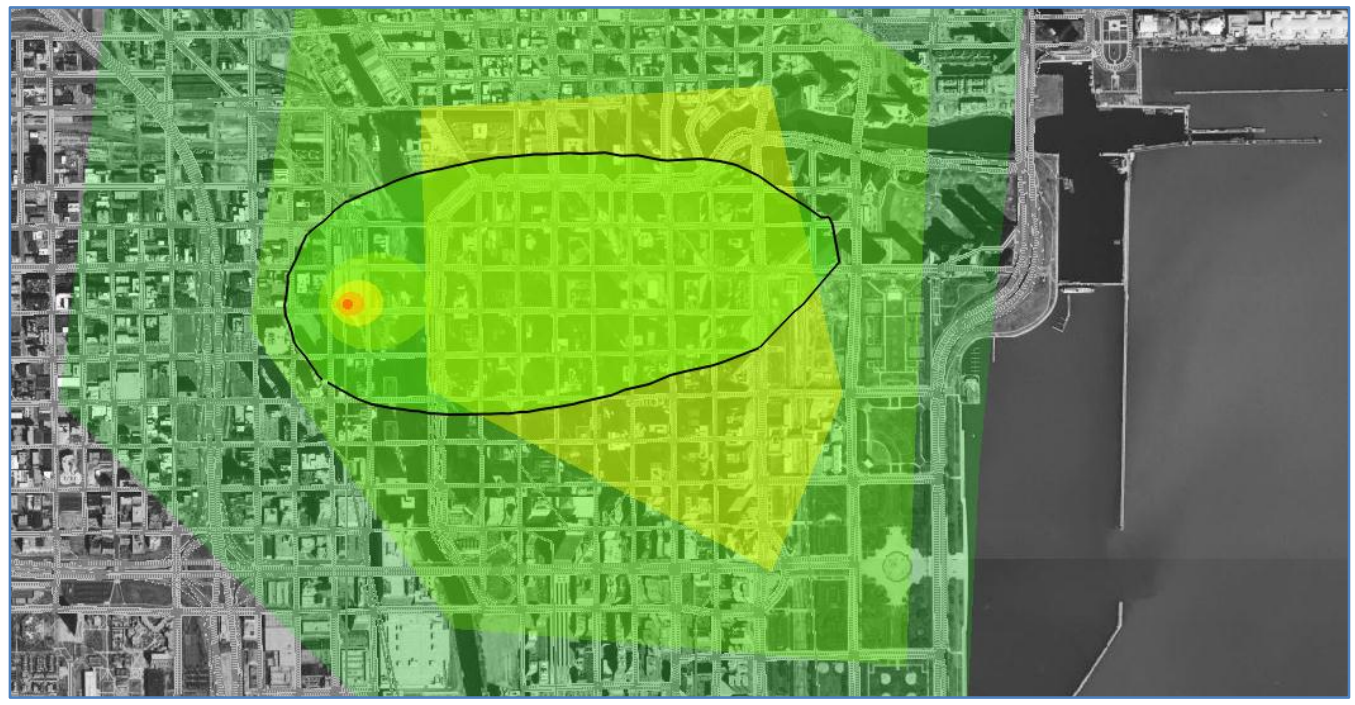




\subsection{Evacuation Start Time and Scenario Saving}

The Evacuation Start Time and the Scenario Saving function is used to define the start time of an evacuation scenario and to save the

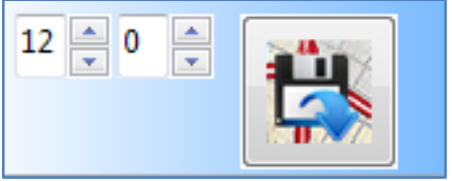
scenario data to corresponding control files. The Dispersion Area function consists of one button and a selection list.

The Evacuation Start Time Selection List is used to define the start time of an evacuation scenario where the first field is the hour and the next field is the minute. The Evacuation Scenario Saving Button is used to save the defined evacuation scenario to corresponding control files. The output control files are as follows.

- EvacDemand.ctl

- EvacNet.ctl 


\subsubsection{RTSTEP Visualizer}

\subsubsection{Introduction}

RTSTEP Visualizer is a TRANSIMS visualizer that was developed as a part of the RTSTEP program. It is designed to assist the modeler in interpreting the output data of TRANSIMS using creative visual paradigms. It has three core components. The heart of RTSTEP Visualizer is the visualization of dynamic traffic data such as congestion or vehicle trajectories. Another component is the ability to create a play list of cinematic sequences from the dynamic traffic data. Finally, RTSTEP Visualizer allows the creation of a dynamic traffic evacuation scenario with a "dirty bomb" nuclear explosion plume superimposed.

The purpose of this User's Guide is to provide documentation that will allow users to be able to install and use RTSTEP Visualizer. The guide was written so that a beginner would be able to follow through the instructions and be able to use the application to visualize a TRANSIMS project; however, it is assumed that the user has a good grasp of the TRANSIMS concepts and is able to use included tools to create a snapshot and associated data files using the Transims microsimulator.

It should be noted that this manual relates to RTSTEP Visualizer Version 2. A previous version of RTSTEP Visualizer was written using the Python programming language and a RTSTEP Visualizer Version 1 manual was created describing that version. RTSTEP Visualizer version 2 was written using $\mathrm{C}++$, the $\mathrm{C}++$ multithreading libraries, and WX-Widgets. This paradigm has the advantage of significantly improving performance and potentially capitalizing on OpenGL for 3D vehicle models. There are significant differences in the GUI between Version 1 and Version 2, and thus the user should be cognoscente of that fact when referring to this Version 2 manual.

\subsubsection{Getting Started with RTSTEP Visualizer}

The transportation Research and Analysis Computing Center (TRACC) has developed a program that contains two parts: RTSTEP Visualizer for visualizing transportation networks and the RTSTEP Evacuation Planner. The two programs will come packaged as one program called RTSTEP Visualizer. Although a knowledgeable developer can create their own "Average Day Files" as input, the packaged version of RTSTEP Visualizer will contain a set of these files for the greater Chicago area that have already been run through Transims to produce a snapshot file that can be visualized using RTSTEP Visualizer. In order to produce a snapshot file that contains evacuation data, the RTSTEP Evacuation Planner will have to be run as described in the following section.

The current version of RTSTEP Visualizer will only run on a Windows machine. Future versions will be made platform independent so that they can also run on a Mac OSX computer. RTSTEP Visualizer is a fairly CPU intensive application. The faster the processor that you have, the better the application will run. We recommend at least a $2 \mathrm{GHz}$ dual core CPU with a minimum of 2 Gigabytes of memory. 


\subsubsection{The RTSTEP Visualizer GUI}

\subsection{Starting the GUI and Loading a RTSTEP Visualizer Project}

Find the file called RTSTEP Visualizer.exe (either through the start menu, desktop icon, or within the TRANSIMS directory) and double click it. The RTSTEP Visualizer GUI should start to run on your computer (see Figure 7.35).

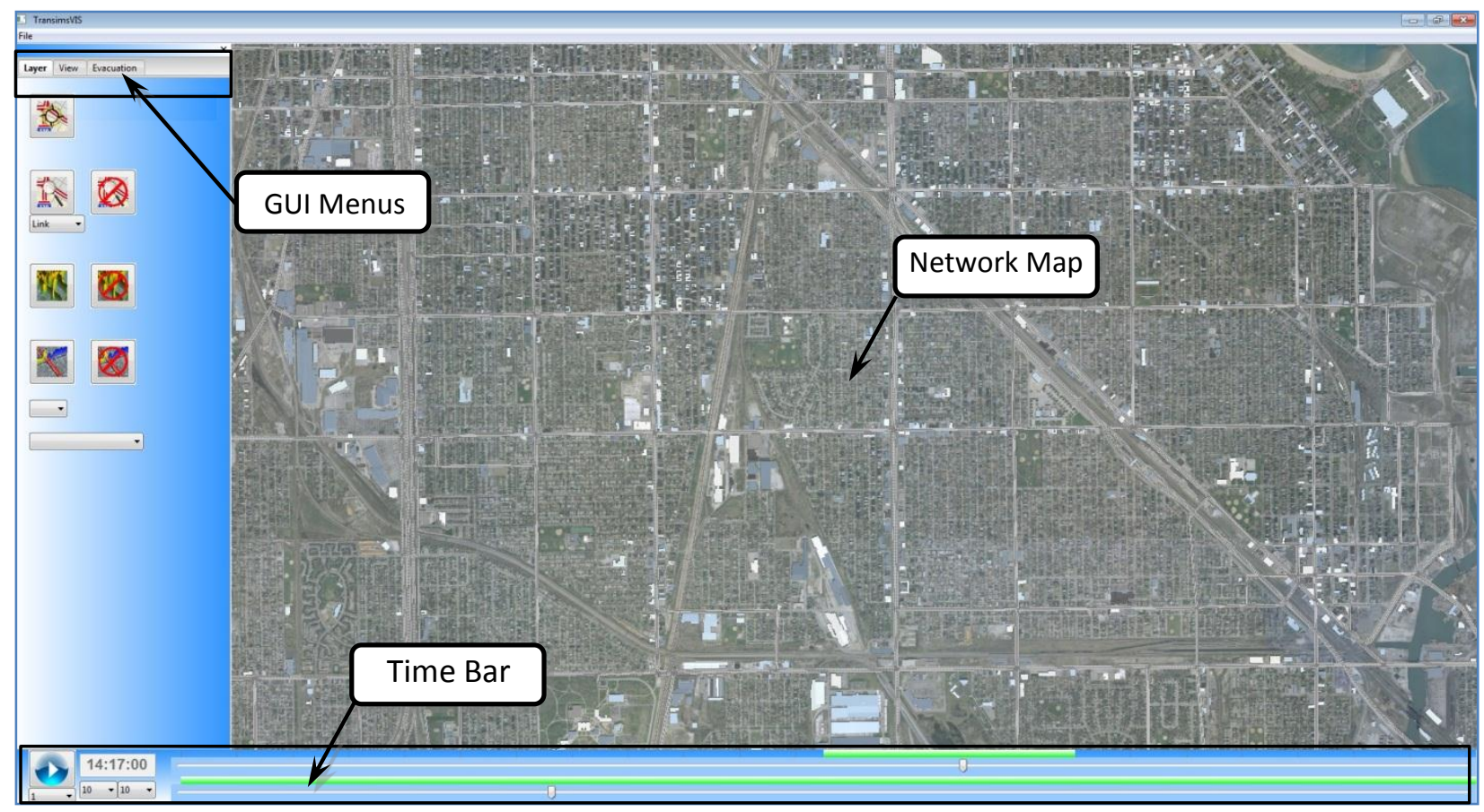

Figure 7.35. The GUI will have three frames: a Map Options Frame on the left, a frame showing the network on the right, and a TimeBar frame at the bottom.

\subsection{TimeBar Controls}

The TimeBar allows you to control the visualization of the network. The "Play" and "Pause" function can be initiated by the single button at the lower left of the TimeBar that allows you to toggle between playing and stopping the visualization. At the bottom of the TimeBar window are two sliders; the top slider designates the hour of the simulation while the bottom one designates the minutes of the hour. As you start the visualization by clicking the start button, the sliders will begin to move toward the right indicating time as it increases.

The actual time of a particular moment in the visualization is shown in the "Time" window located just to the right of the start/stop button of the TimeBar. You can manually move the time slider using your mouse to any time within the simulation. The slides provide for a complete 24-hour simulation although the snapshot file may only cover a portion 
of the 24-hour period. A green indicator is present over the sliders to indicate that portion of the 24 -hour period for which simulation data is present.

There are three buttons that control the rate at which the simulation is played. These three buttons are located at the lower left of the TimeBar and are, from left to right, the:

\section{1. "Frames per Second"}

2. "Interpolations per Step", and the

3. "Simulation Time per Step".

The frames per second is the number of frames per second that the visualizer tries to achieve or to put it another way the number of frames per second that the visualizer tries to render. The number of frames per second can be selected from the pull down list ranging from a low of 1 up to 60 frames per second or even "Max" which is the fastest that the program will allow frames to be rendered. The frames per second should be kept as low as practical to ensure that the display of frames is readable given the ability of your particular computer to render frames.

A frame is the display of one iteration of the visualization. Each step is further broken down into one or more iterations. The value of the time for each frame is controlled by a combination of the number of interpolations per step and the simulation time per step. Interpolations provide a smooth transition between individual steps created by the microsimulator when the snapshot file is created. The default is for a step size of 1 second and 1 interpolation per step. With both the number of interpolations per step and the simulation time per step set to 1 , every frame would represent 1 second of the simulation process.

The number of interpolations per step can range from 1 to 10 and the simulation time can range from 1 to 900 seconds. As the number of interpolations per step increases, the simulation time per frame decreases proportionally. As the simulation time per step increases, the simulation time per frame decreases proportionally.

\subsection{GUI Menus}

There are three GUI menus that are shown at the top of the Map Options window:
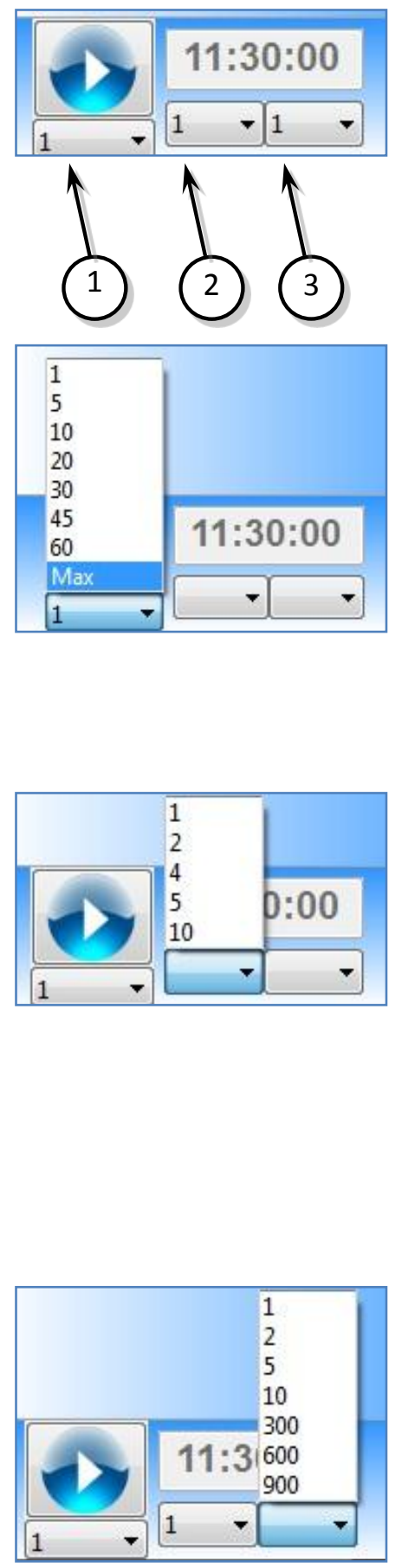
- Layer

- View

- Evacuation

These three menu control options can be used for designing and controlling the visualization of the network; adding waypoints to the network; and configuring and viewing an evacuation scenario. When one of the menus is selected, the map options windows shows the various options for each menu. Each of these menus is described in more detail in the section "Menu Options" below.

\subsubsection{Navigating and Visualizing the Network}

Now that you have a network loaded into RTSTEP Visualizer and have located the basic canvas controls and menus, you can begin to navigate through the network. First left click on the network with the mouse to select the network pane. Navigation is then handled by panning with the left mouse button, zooming in and out with the mouse wheel, and 3-dimension rotation by holding and dragging with the right mouse button. It should be noted that it is more difficult to navigate with a computer that only has a touch-pad mouse. A physical two-button mouse with a mouse wheel is recommended.

The second by second simulation can be viewed by clicking on the "Play" button at the bottom of the screen. Once started, the vehicles can be seen traversing the network. Also, you may have to zoom in to a higher level of detail to observe the cars and make sure that the time on the time bar slider is in a green area where traffic data exists. As the simulation time increases, the sliders (hours and minutes) at the bottom of the window will move toward the right. The visualization can be stopped at any point in time by toggling the "Start" button. 


\subsubsection{Menu Operations}

There are three main menus for controlling the display of the traffic on the network:

- Layer Menu

- View Menu

- Evacuation Menu

\subsection{Layer Menu}

Once the Layer Menu has been selected, you should see the Map Options Frame to the left of the window. The options for the Layer menu will allow you to do the following functions:
1. Navigation
2. Identification
3. Heat Plot
4. Link Plot
5. Probe Vehicles
6. Shape Files

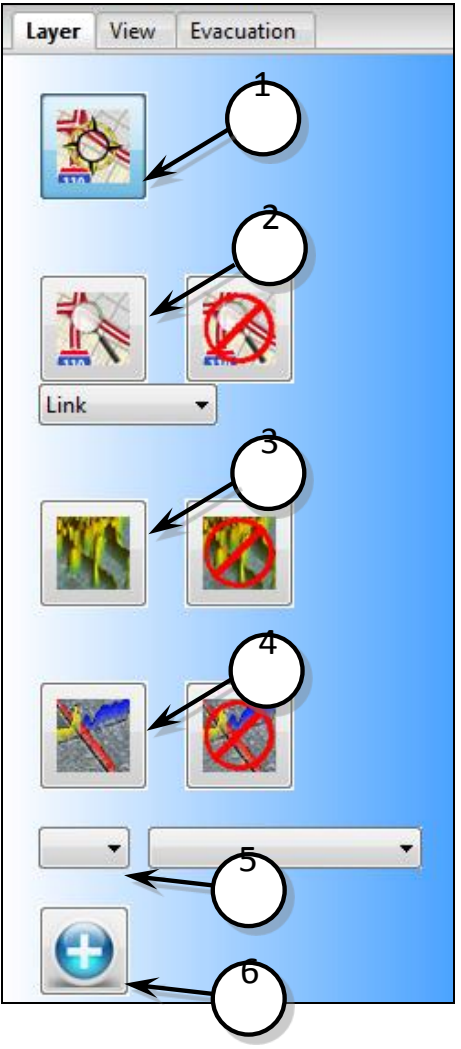

\subsection{Navigation}

The top button in the Map Options Frame is the Navigation button. It should be noted that once the user selects one of the other options (Identification Heat Plot, Link Plot or Shape Files), the navigation function of the mouse is disabled, and the mouse is used for

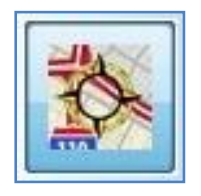
the selected option. In order to return the mouse to be used for navigation, the Navigation button can be clicked thus cancelling the other option(s). 


\subsection{Identification}

Identification is controlled by a combination of the two buttons and the pull down list located just under the Navigation button. There are four different "elements" that can be identified. They can be selected in the identification pull down list as any one of the following:

1. Link

2. Node

3. Vehicle

4. Shape Layer

Links and nodes are as defined for the project's network. Vehicle relates to the vehicles that move in the simulation. Once a type of identification has been selected, the user needs to click on the left hand button (the enable identification button) located immediately above the identification pull down list in order to allow identifications. When the button has been clicked, a new frame, an identification frame, will open up at the right hand side of the display. The user can then move the mouse around on the network and the information for the selected item will be highlighted on the display and its information will be shown in the identification frame. As the mouse is moved, the information will change as the mouse passes over one of the elements of the type selected.

As the mouse passes over an element (link, node, or vehicle depending on the type) selected, the information for that element will appear in the identification frame. As the mouse is moved to a new element, the information for that element will appear. If the mouse is left clicked, the information for that element will remain in the identification frame until a new element is selected (left clicked).

To clear the identification option, return the mouse to the Navigation mode and to remove the identification frame; the user can either click the Clear Identification button located next to the Identification button or click the Navigation button as described in 7.5.4.5.1.1.
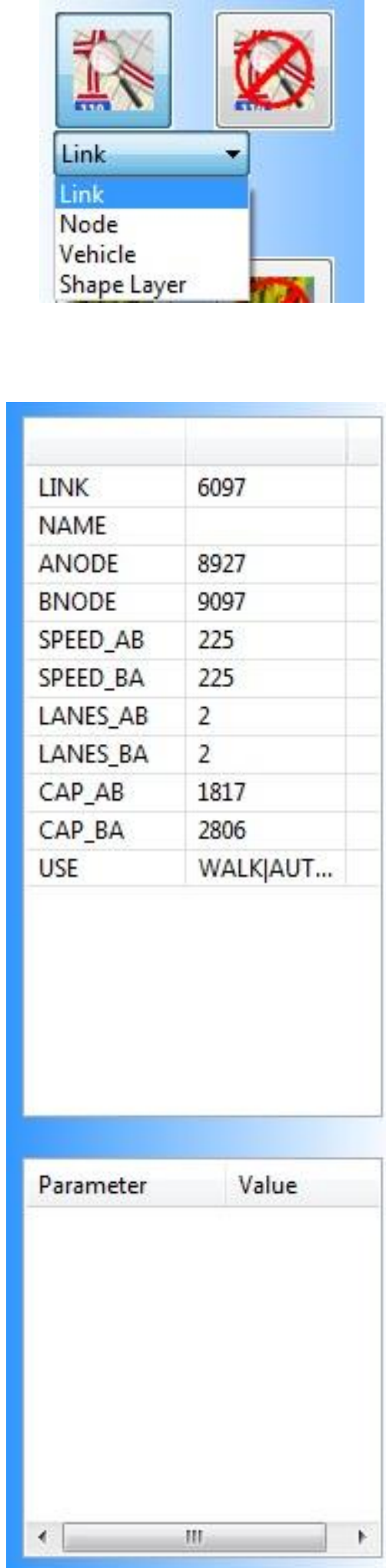


\subsection{Heat Plot}

The Heat Plot option is controlled by the two buttons located directly below the identification buttons; they are the Heat Plot button (the button on the left) and the Clear Heat Plot button (the button on the right). First click the Heat Plot button and then select an area of the network by holding down the left mouse button on the network and dragging the mouse to extend the sides of the heat plot shown by a transparent red area. Release the button when the desired area has been selected. When the simulation is played, the heat plot will be displayed.

Cooler areas will be shown in a light green color and will be essentially transparent with gradually increasing heat shown as turning to red and being opaque for the hottest areas. Rotating the display may help visualize the heat plots.

There are several specific heat plot paradigms that can be selected. They can be selected using the heat plot pull down list. These are based on statistics output from the microsimulator. Speed Ratio is basically a measure of congestion. It indicates a low speed ratio of the vehicle as compared to the speed limit thus causing congestion and therefore heat. Time Difference is a measure of how far along a person is in their trip as compared to where they should be. In other words it is a measure of how far behind schedule they are. Waiting time is a measure of how long a vehicle is in one spot or basically stopped. User defined is not yet developed and would allow the user to define a different paradigm.

To clear the Heat Plot option and return the mouse to the Navigation mode, the user can either click the clear Heat Plot button located next to the Heat Plot button or click the Navigation button as described in 7.5.4.5.1.1.

\subsection{Link Plot}

The link plot is basically the same as the heat plot but measures congestion rather than heat. It is a better option for getting more precise data over a smaller area of the map. The bottom two buttons in the map options frame control the link plot. First click the left hand button and select an area on the map by holding down the left mouse button and extend the area by moving the mouse diagonally creating a purple area for the link plot. Release the button when the desired area has been selected. When the simulation is played, the link plot
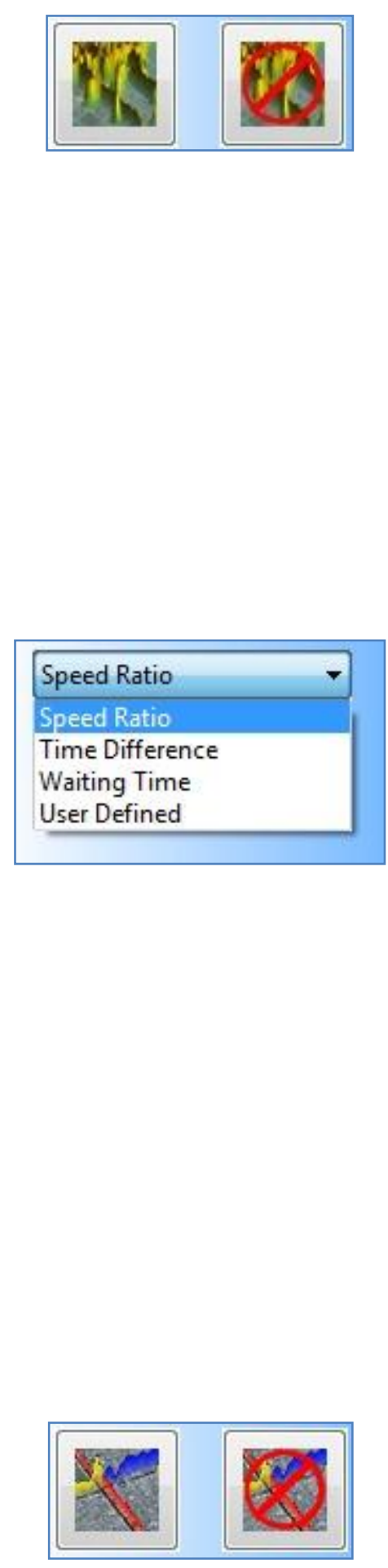
showing congestion will be displayed.

There are several specific link plot paradigms that can be selected. They can be selected using the same pull down menu as used for the heat plot above. These are based on statistics output from the Microsimulator. Speed Ratio is basically a measure of congestion. It indicates a low speed ratio of the vehicle as compared to the speed limit thus causing congestion. Time Difference is a measure of how far along a person is in their trip as compared to where they should be. In other words it is a measure of how far behind schedule they are. Waiting time is a measure of how long a vehicle is in one spot or basically stopped. User defined is not yet developed and would allow the user to define a different paradigm.

By zooming in on the map, the detailed congestion is shown for individual links on the map. The color of the plot has significance as to which way the traffic is flowing: blue for north bound traffic, yellow for south bound traffic, red for east bound traffic, and green for west bound traffic.

To clear the Link Plot option and return the mouse to the Navigation mode, the user can either click the Clear Link plot button located next to the Link Plot button or click the Navigation button as described in 7.5.4.5.1.1.

\subsection{Probe Vehicles}

Probe vehicles provide a mechanism to show a selected percentage of vehicles traveling on the network even though the network has been zoomed out so far that normal sized vehicles cannot be resolved on the display. As you zoom out, the vehicles are normally displayed with a decreased pixel length. Eventually they become too small to be seen. Probe vehicles however, remain displayed as visible dots even when zoomed out.

The percentage of vehicles that are displayed as probe vehicles can be selected on the probe vehicles pull down list as $0,1,5,10$, or 100 percent (located near the bottom of the Map Options Frame). If " 0 " is selected, no probe vehicles are shown. If " 100 " is selected, all vehicles are shown as probe vehicles. " 1 ", " 5 ", and " 10 " allow you to select percentages in between zero and one hundred percent. When zoomed out so that the probe vehicles show as dots, the color of the dots indicates the speed of the probe vehicle. The color changes from black to orange, to yellow to green as the vehicle speeds up.
Speed Ratio Speed Ratio

Time Difference

Waiting Time

User Defined 
A black dot would thus indicate severe congestion. When zoomed in, all of the vehicles are shown in detail.

\subsection{Shape Files}

Users also have an option to add shape files as additional layers to the existing network view. Clicking the left mouse button on the Plus icon opens up the file manager for locating various shape files. Once a file is loaded, new menu options become available to the user:

1. The color and opacity settings,

2. shape file list, and

3. shape file attribute list.

Multiple shape files can be loaded at once. They can be activated and deactivated by using the check box located next to the file name in the file list box. They can be unloaded by clicking the Minus button.

For each layer, a distinct color can be selected by clicking on the color bar. The first set of arrow buttons next to the color bars is used to control the opacity of the layer. By clicking the top arrow, the user can turn the layer opaque. Clicking the down arrow makes it transparent. The second set of larger arrows on the right is used to control the marker size. The up arrow increases the size of the marker; the down arrow decreases it.

Each layer can be displayed with different marker shapes. The menu underneath the color bars allows for selecting one of the available shapes for a given type of data. The point data can be displayed as points, pyramids, cubes, or prisms. The line data can be displayed as lines, ribbons, or paths.

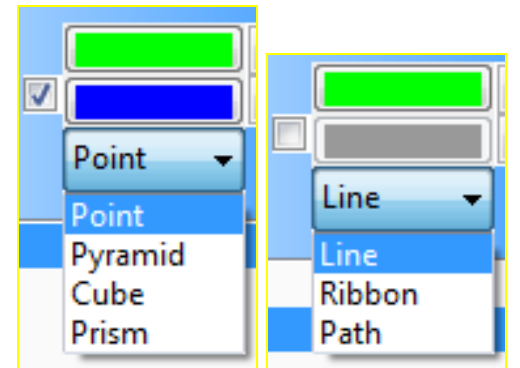


By checking the check box located to the left of the second color bar, a user has an option to activate a two-color gradient for the attributes of the shape files. The two selected colors will denote how minimum and maximum values of the given component is displayed. Values in between them will be displayed with an interpolated color. As an example, a regional node shape file with a gradation of the $y$ -

\begin{tabular}{|l|l|}
\hline NODE & $\mathbf{4 5 3 9 1 7 1 . 0}$ \\
\hline X_COORD & \\
\hline Y_COORD \\
Z_COORD \\
\\
\end{tabular}
coordinate is shown in Figure 7.36.

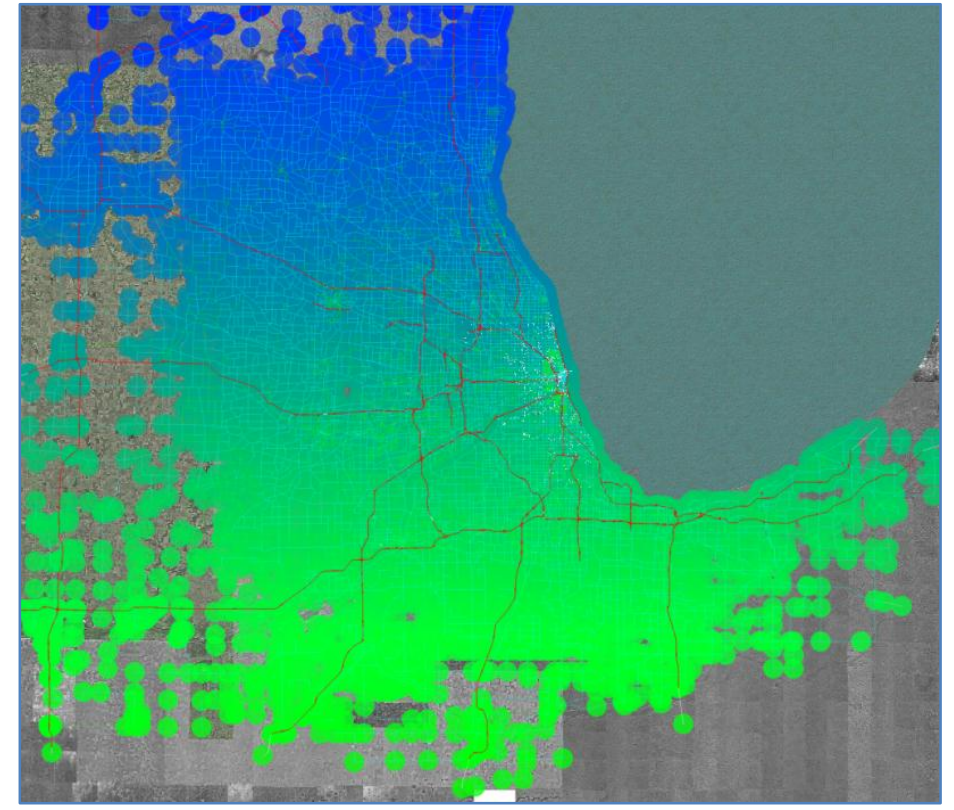

Figure 7.36. An example of shape file displayed with the color gradation. 


\subsection{View Menu}

Once the View Menu has been selected, you should see the Options for the View Menu in the Map Options Frame on the left side of the display. The options for the View Menu will allow you to set waypoints in the simulation and create cinematic sequences. The options frame consists of two large boxes and three buttons. You first need to configure a series of waypoints that will be shown in the top box. Once you have configured the desired waypoints for your sequence, you need to select them (or some of them) and copy them to the lower box. This box defines the sequence that will be followed when you follow the waypoints and play the sequence.

First you need to navigate on the map to the initial waypoint be moving to the desired time for the initial point and then zooming, panning, and rotating the map as desired. By clicking on the camera button, the initial waypoint will be entered into the top box. By clicking on that waypoint, and then clicking a second time (do not double click, rather click slowly), you can enter a label that has some significance to you to identify that waypoint. You can then advance to a new time in the simulation and repeat the process to create additional waypoints.

Once all of the desired waypoints have been created for the cinematic sequence you want to create, you can highlight each one and click the Plus button to add the waypoint to the sequence that will copy it to the lower box. You can remove any waypoint from the sequence by highlighting it and clicking on the Minus button.

To play the sequence, click the Follow Waypoints button and the start the sequence by clicking the Play button. The cinematic sequence will start to play starting from the initial waypoint and continuing through to the last waypoint. The simulation will be played sweeping through from one waypoint to another until the last waypoint is reached.
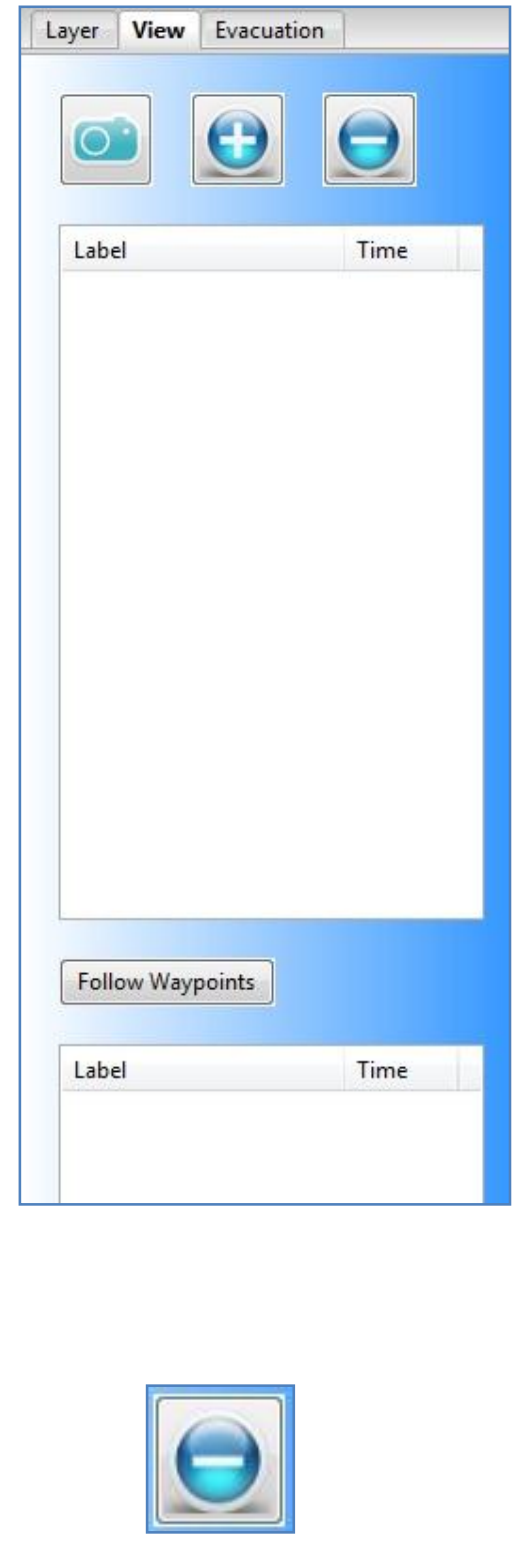

\subsection{Evacuation Menu}

The Evacuation Menu is explained in detail above in Section 3, the RTSTEP Evacuation Configurator. 


\subsubsection{Making a Movie of Your Project}

To accomplish the task of making a movie, a third party software application is required to capture screen shots and compose them into a movie. One of the applications that we have used is HyperCam 2. First you will have to down load and install your application. HyperCam 2 requires that you:

- Select a region of the screen that you want to capture- this is typically the network frame of the visualizer.

- You need to select a codec to encode the captured frames- we typically select the XVID codec.

- Select the frame rate for capturing- we typically use 10 frames per second.

- Select a location to store the encoded video file

Once the capture application is set up, you need to decide on the video sequence that you want to visualize. Typically you will want to set up a cinematic sequence as described in "View Menu", section 4.3.2 above. You can select way points as desired and include any of the effects such as Heat Plot, Link Plot, and Probe Vehicles and include any zooming or panning that is appropriate.

Once you have configured the capture application and set up the sequence to be visualized, you can click the start button on the application in order to start capturing frames and then click the start button on the visualizer in order to start the video sequence. When the video sequence has reached the desired end point, click the stop button on the capture application to complete the encoding of the video clip.

Capture applications have a limit on the rate that they can capture frames. This may be in the 5 to 10 frame per second range depending on the particular software application and the computer it is running on. This is slower than one would like and limits the rate at which the simulation can be played. The "Frames per Second", "Interpolations per Step", and "Simulation Time per Step" (see "TimeBar Controls", section 4.1.2 above) must thus be chosen judiciously and the process may require a little trial and error to get the parameters set just right.

There is a new feature that is currently under development and should be implemented soon. This feature would allow the user to output frames during the course of visualizing the network. The output that would be stored would be individual frames. Another application would have to be run to post process the frames and integrate them into a continuous movie clip. An application like FFMPEG would be suitable for this activity. The feature will be implemented so that a button can be clicked to enable visualized frames to be stored and to be toggled off to stop the process. This feature will provide a movie clip that will run much smoother than a screen capture process. 


\section{Work for Others - Computational Mechanics Research and Support for Aerodynamics and Hydraulics at TFHRC}

\subsection{Computational Fluid Dynamics}

During the fourth quarter modeling and analysis of the separation of flow at the leading edge of a flooded bridge deck was begun to aid in the development of an enhanced approach for evaluating scour due to submergence of bridge decks during floods. Modeling of flow through culverts for fish passage continued with the addition of a porous media model to capture the effects of large diameter gravel in the bottom of the culvert. TFHRC provided geometry files for their wind tunnel laboratory and a new CFD model was developed to analyze flow in the wind tunnel and the room under a variety of flow conditions including with and without furniture. A CFD model using the sliding mesh capabilities of STAR-CCM+ with multiphase droplet tracking was developed and tested for application to the analysis of road salt spray from large truck traffic under bridges with weathering steel.

\subsubsection{CFD Analysis of Flow Separation under an Inundated Bridge Deck for Application to Pressure Scour Evaluation}

An update to the submerged-flow bridge scour evaluation procedure in HEC 18 [1] is being prepared by TFHRC. The approach to scour hole estimation assumes that the scour process will enlarge the area under the bridge until it is large enough to pass the flow with a condition of critical shear stress at the bed. The bridge deck is a bluff body in the flow and flow separation will normally occur at the upstream bottom edge of the submerged bridge deck. The separation zone under the bridge restricts the area open to flow under the deck and is therefore an important parameter in conservatively predicting the depth of the scour hole. A set of CFD simulations were performed to investigate the relation between the initial opening height under the submerged deck before scour and the thickness of the separation zone, and one test was done to see if the thickness of the separation zone changed during the scour process. 


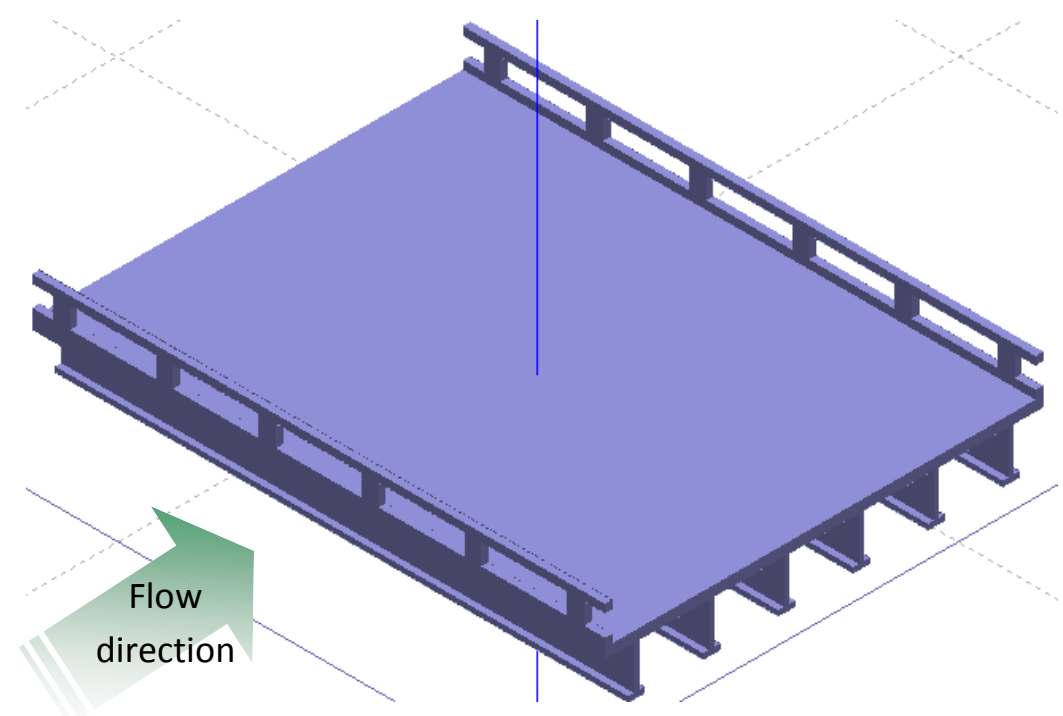

Figure 8.1: Bridge deck geometry

The bridge deck geometry is shown in Figure 8.1, and a schematic of the flow domain is shown in Figure 8.2. To reduce computer time and eliminate the effects of the flume side walls, the simulations were performed using a section of the bridge deck cut through the middle of a post and running half the distance to the center of the next post. This geometry is shown in Figure 8.3.

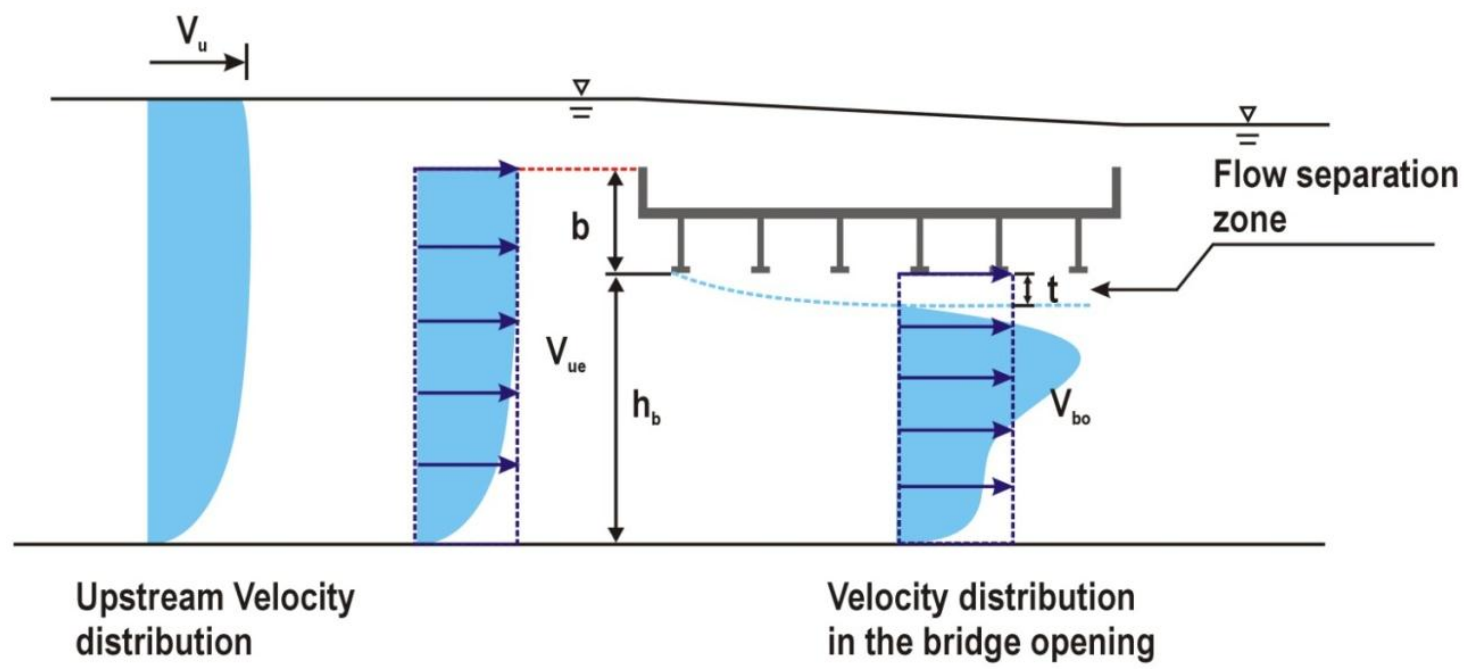

Figure 8.2: Schematic of flow domain for separation zone simulations 


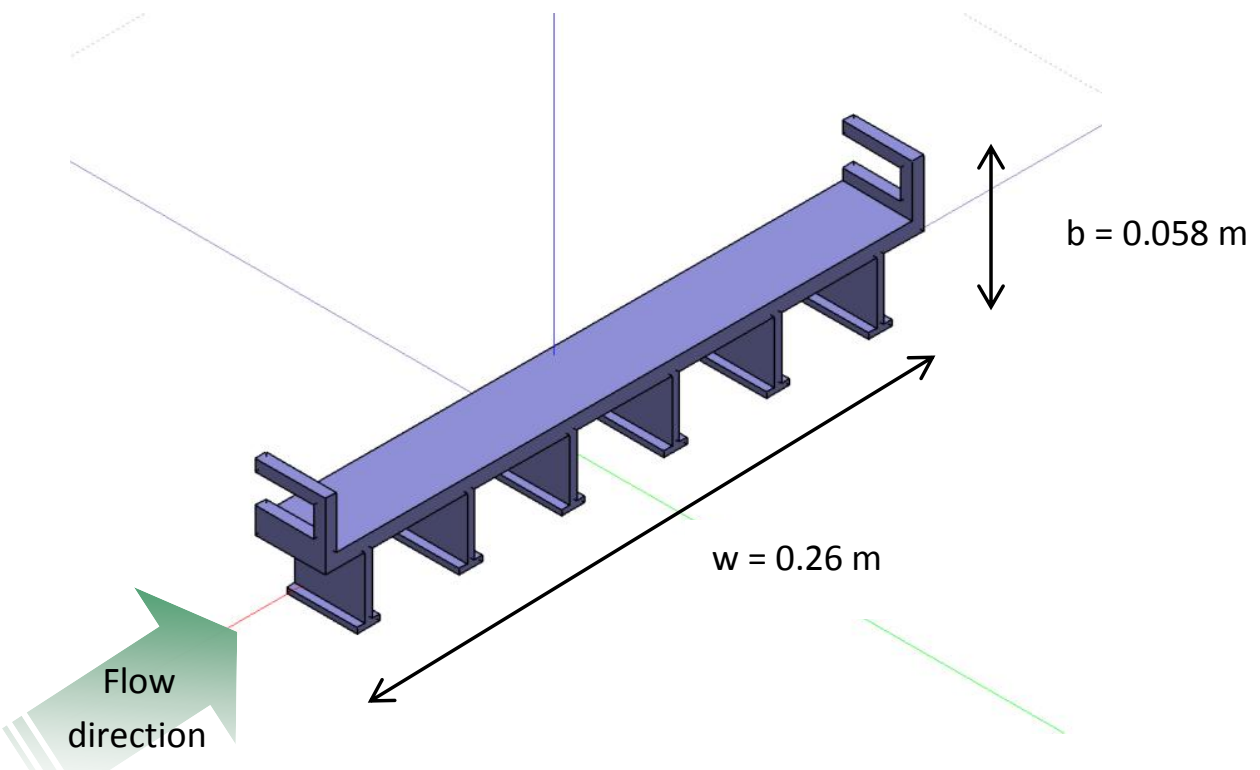

Figure 8.3: Symmetric section of bridge deck from center of a railing post to halfway to the next post

Symmetric boundary conditions were used on the cross stream sides of the domain. The simulations were done as single phase flow with a flat water surface using a symmetry boundary condition at the surface. Previous tests have been done using the multiphase VOF model for free surface flow, and the flat surface assumption is good except in the case with the bridge deck very close to the surface but still overtopped. The inlet boundary was taken to be a uniform velocity located just at the outlet of the honeycomb in the TFHRC scour flume. The honeycomb is a flow straightener that also strips off boundary layers, and therefore the uniform inlet velocity is a reasonably good assumption applied at this position.

\subsubsection{Separation Zone Before and After Scour Hole Formation}

To determine the effect of scour hole formation on the size of the separation zone below the bridge deck, the maximum thickness of the separation zone before and after scour was calculated using an existing scour model that does not include sediment transport. The model is based on a TFHRC scour experiment with water depth of $25 \mathrm{~cm}$, bridge height above the bed, $h_{b}$, of $16 \mathrm{~cm}$, and mean inlet flow velocity of $0.482 \mathrm{~m} / \mathrm{s}$. Figure 8.4 shows the velocity field under the deck for the initial un-scoured flat bed. High velocity under the deck caused by the deck blockage in the flow causes scour of the bottom. The velocity distribution under the deck after scour in shown in Figure 8.5: plotted using the same scale as the flat bed pre-scour condition. The reduced velocity under the deck is apparent. With the scour hole present the maximum shear stress under the deck is reduced to the critical shear stress. 


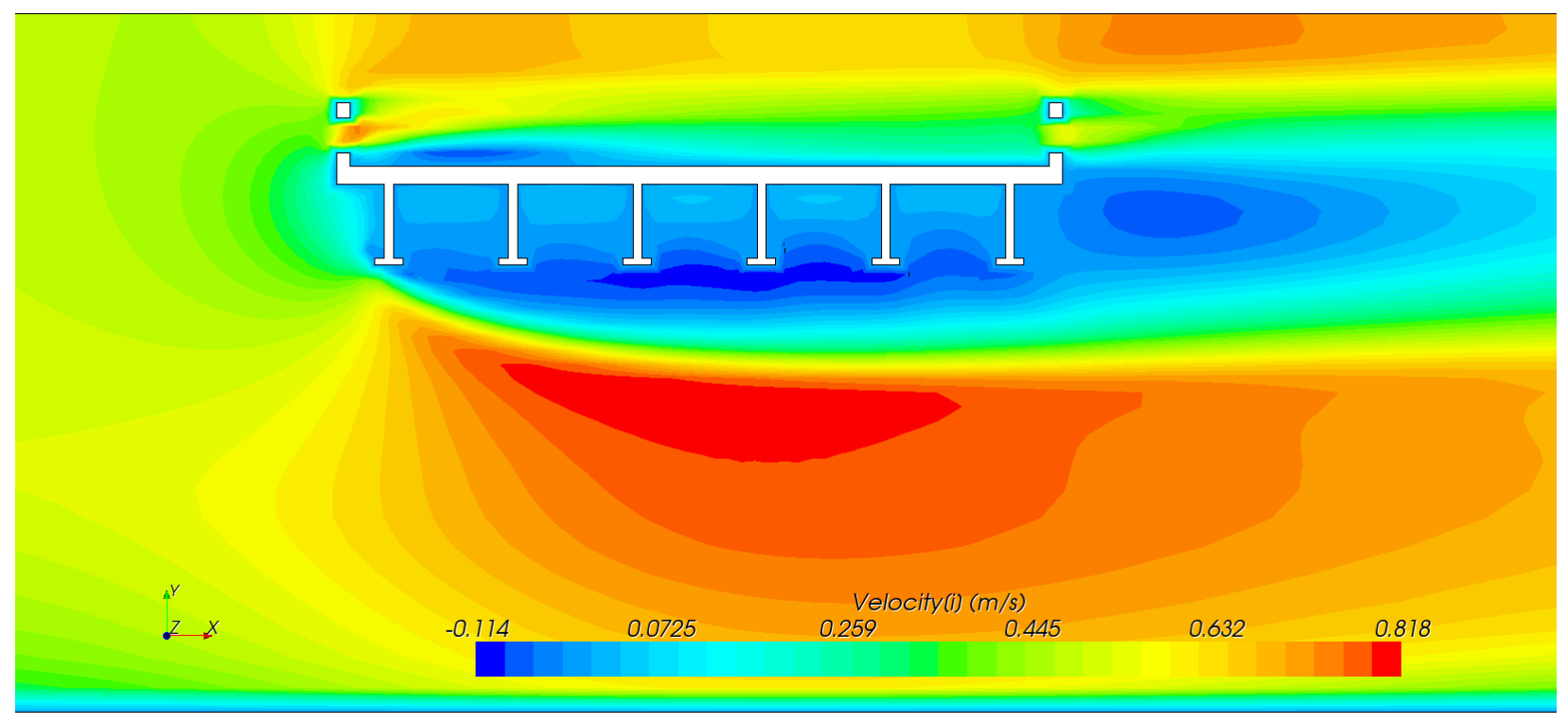

Figure 8.4: Velocity field under bridge deck before scour

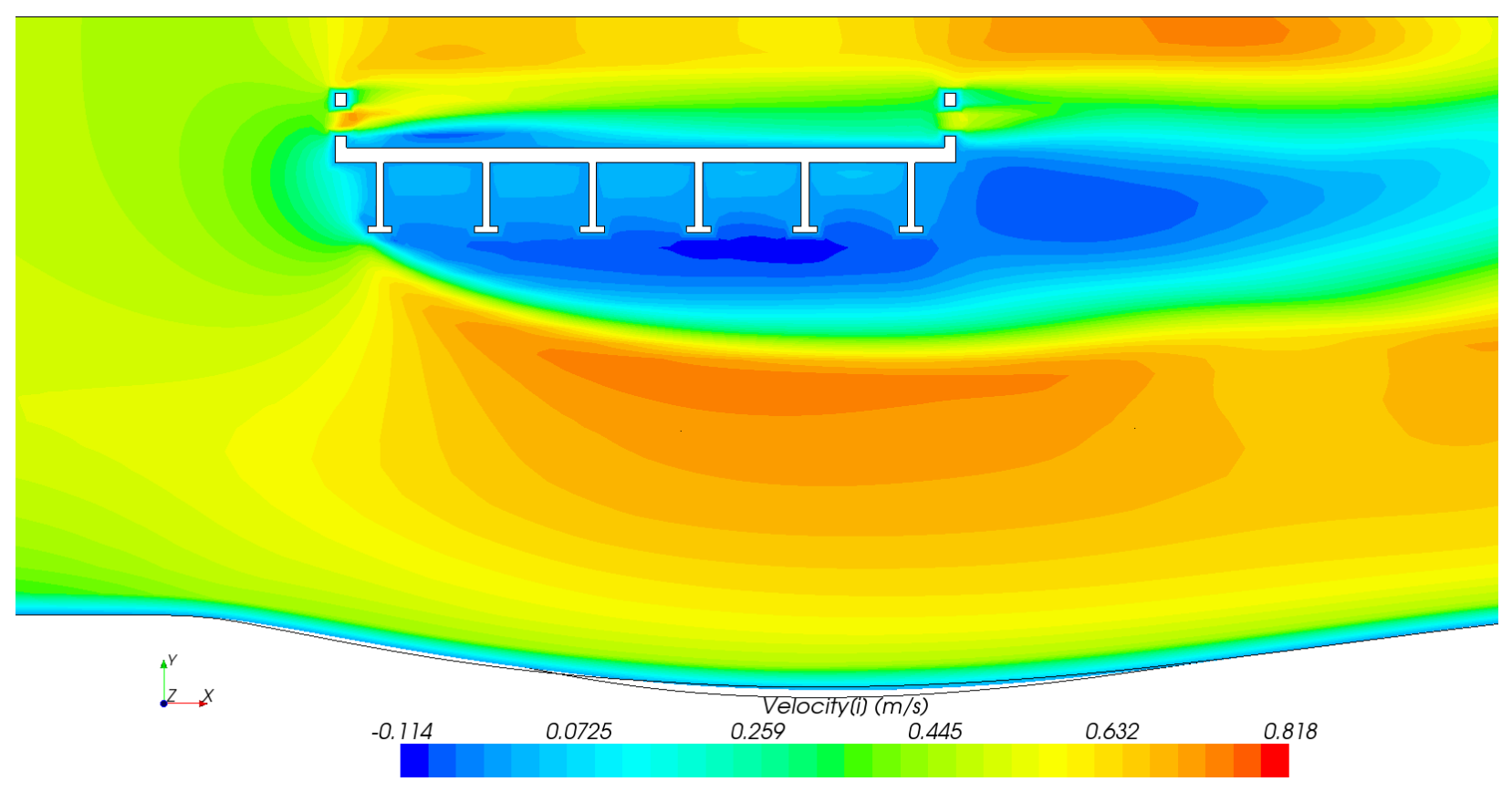

Figure 8.5: Velocity field under bridge deck after scour

Velocity profiles in the upstream of the deck (blue line) and $15 \mathrm{~cm}$ under the deck (red line) before scour are shown in Figure 8.6. The negative $x$-direction velocity due to recirculation in the separation zone beneath the deck can be seen. 
Velocity Profiles (Mean Inlet Velocity $=0.482 \mathrm{~m} / \mathrm{s}$ )

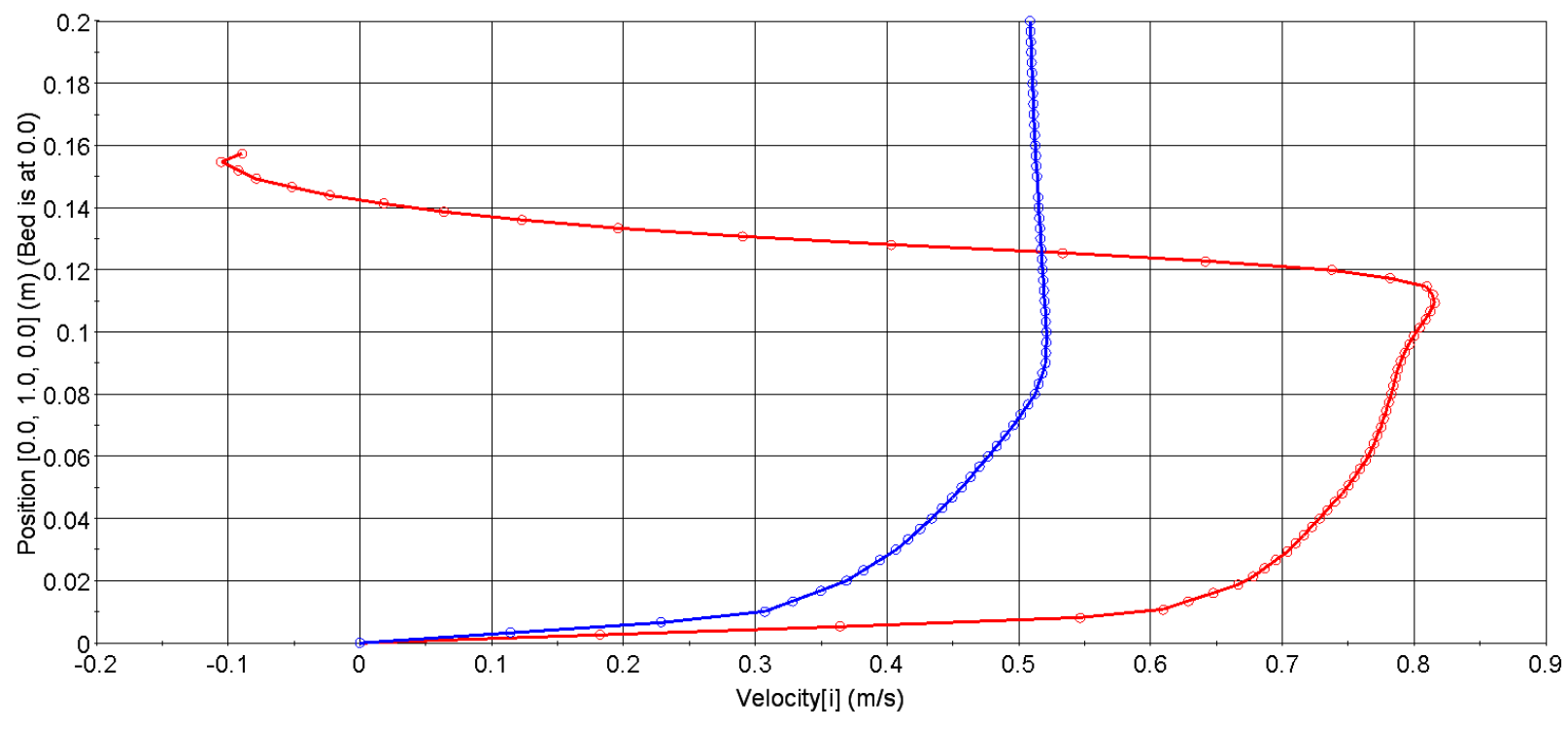

$\ominus$ line-probe-15-cm-under-deck $\ominus$ line-probe-upstream

Figure 8.6: Velocity profiles upstream and $15 \mathrm{~cm}$ under the deck

The size of the separation zone for both the pre and post scour conditions is determined by computing the streamline that starts from the leading edge at the bottom of the first I-beam. A plot of this streamline for the pre scour condition is shown in Figure 8.7. It is colored with the height above the flume bed. The maximum thickness of the separation zone is determined by using a STAR-CCM+ report to find the minimum height of the streamline at the zone boundary above the bed and subtracting the result from the height of the deck above the bed in another report. For the pre scour condition, the thickness of the separation zone is $0.033 \mathrm{~m}$. For the post scour condition, the thickness of the separation zone grows to $0.046 \mathrm{~m}$. Therefore, the larger opening under the deck when the scour hole has formed does lead to a larger separation zone under the bridge deck.

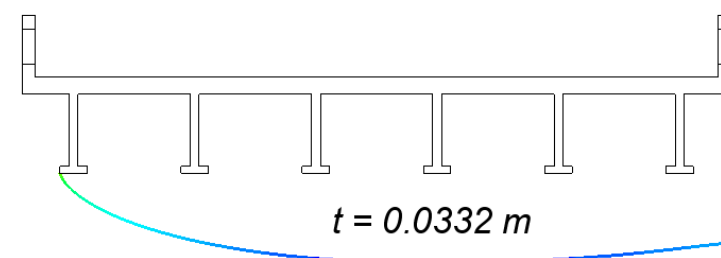

Minimum $y=0.1268 \mathrm{~m}$

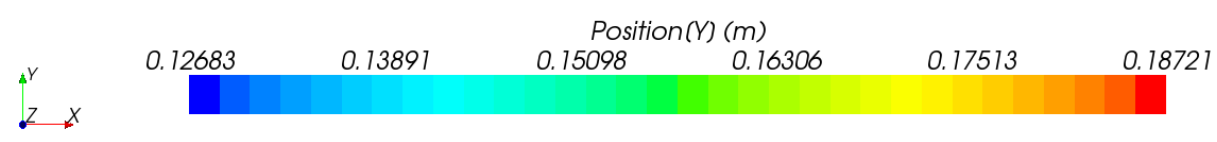


Figure 8.7: Streamline bounding the separation zone under the deck before scour

The qualitative differences in the separation zones for the pre and post scour conditions can also be seen in Figure 8.8 and Figure 8.9. These figures show a set of streamlines seeded from a line upstream of the deck blockage. The stagnation point on the upstream side of the deck is approximately at the height of the road surface, and with more area for flow under the deck after the scour hole forms, the stagnation point moves only slightly downward. The separation zone leading into the wake is larger when the scour hole is present. The maximum thickness increases from $0.033 \mathrm{~m}$ to $0.46 \mathrm{~m}$ and the position of the point of maximum thickness moves from $14 \mathrm{~cm}$ downstream of the leading edge of the first I-beam to $18 \mathrm{~cm}$ downstream of the leading edge.

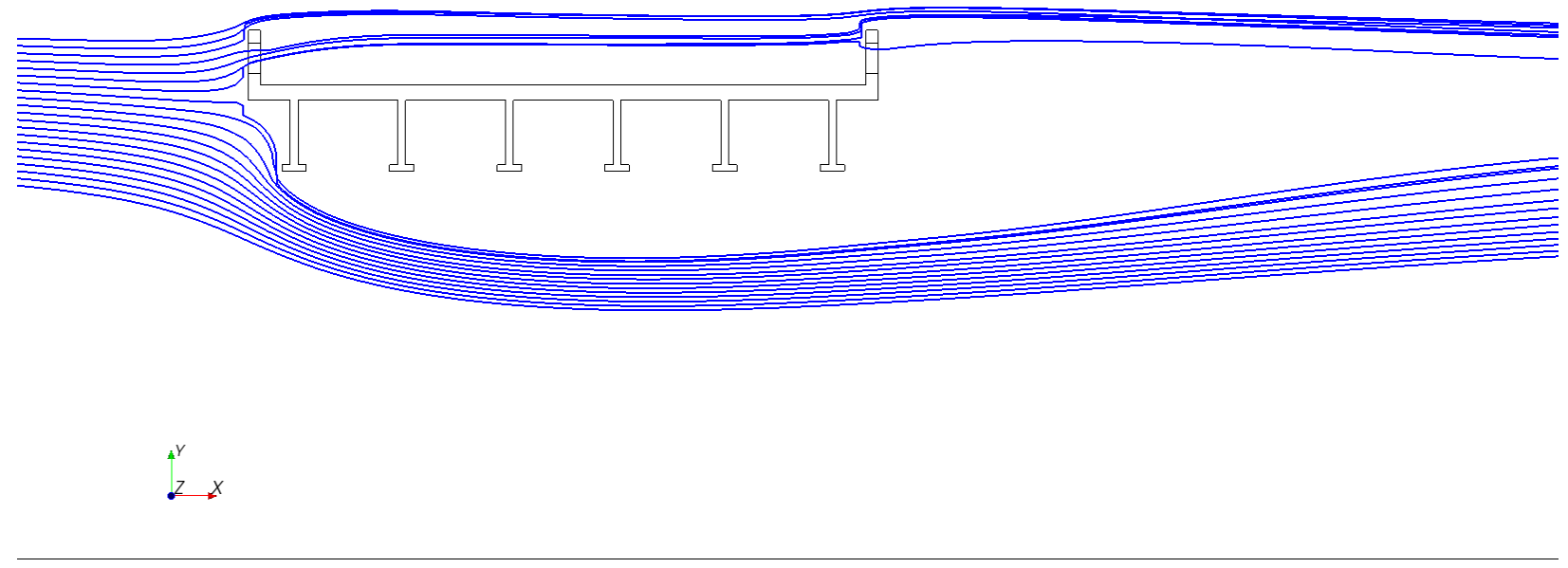

Figure 8.8: Streamlines seeded from the upstream of the deck blockage showing the stagnation point before scour 

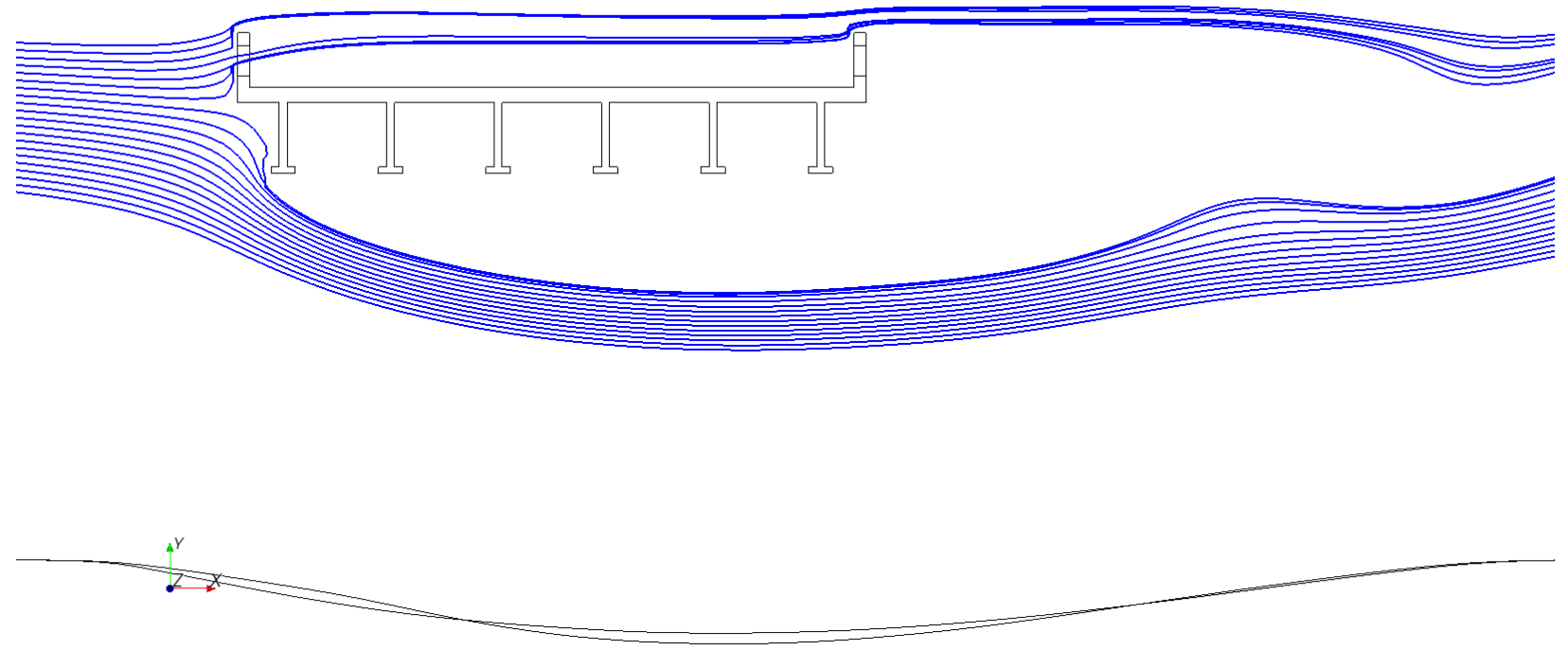

Figure 8.9: Streamlines seeded from the upstream of the deck blockage showing the stagnation point with scour hole present

\subsubsection{Relation between Separation Zone Thickness and Bridge Height above Bed}

A set of simulations were done to test the relation between the height of the bridge deck above the sediment bed and the maximum thickness of the separation zone. For these cases the water depth was $0.5 \mathrm{~m}$, and inlet flow velocities of $0.6 \mathrm{~m} / \mathrm{s}$ and $0.4 \mathrm{~m} / \mathrm{s}$ were used. The set of bridge openings, $h_{b}$, and the maximum contraction created by the separation zone, $\mathrm{t}$, under the deck are listed in Table 8.1. The thickness of the separation zone is not affected much by velocity within the range tested.

Table 8.1 Contraction of flow under bridge deck

\begin{tabular}{|c|c|c|}
\hline $\begin{array}{c}\text { Bridge Opening } \mathrm{h}_{\mathrm{b}} \\
(\mathrm{m})\end{array}$ & $\begin{array}{c}\text { Maximum Contraction } \\
\mathrm{V}_{\mathrm{u}}=0.6 \mathrm{~m} / \mathrm{s} \\
(\mathrm{m})\end{array}$ & $\begin{array}{c}\text { Maximum Contraction } \\
\mathrm{V}_{\mathrm{u}}=0.4 \mathrm{~m} / \mathrm{s} \\
(\mathrm{m})\end{array}$ \\
\hline 0.05 & 0.013 & 0.013 \\
\hline 0.10 & 0.021 & 0.021 \\
\hline 0.15 & 0.026 & 0.027 \\
\hline 0.30 & 0.036 & 0.036 \\
\hline 0.42 & 0.052 & 0.051 \\
\hline 0.47 & 0.048 & 0.046 \\
\hline
\end{tabular}

Figure 8.10 and Figure 8.11 show the bounding streamlines for fully submerged cases where the deck is close to the bed, $h_{b}=5 \mathrm{~cm}$ and where the deck is at the highest point tested that still has significant 
overtopping, $h_{b}=30 \mathrm{~cm}$. The separation zone is relatively thin and short for the case with the deck near the bed, and it grows in thickness with the thickest point moving to the downstream edge of the deck at a deck height of $h_{b}=30 \mathrm{~cm}$.
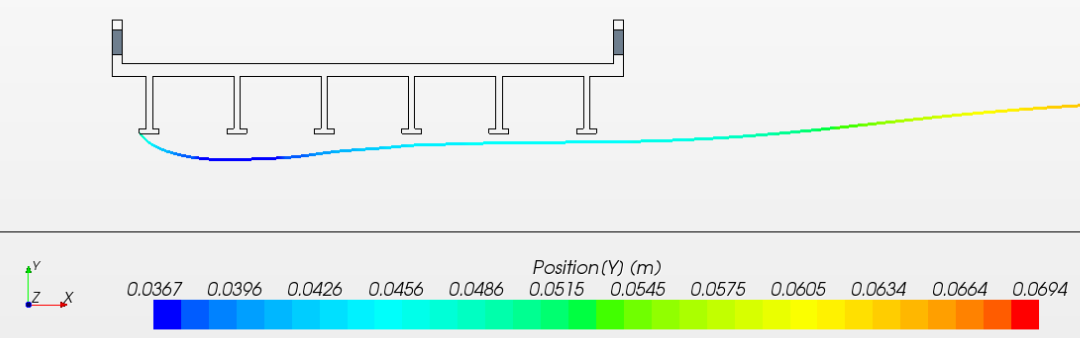

Figure 8.10: Separation zone bounding streamline for $h_{b}=0.05 m$ case

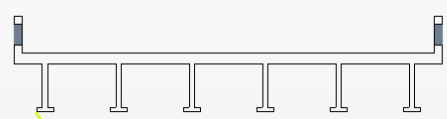

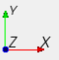

$\begin{array}{llllll}0.367 & 0.374 & 0.381 & 0.388 & 0.394 & \begin{array}{ll}\text { Position }(Y)(m) \\ 0.401 & 0.408\end{array}\end{array}$

$\begin{array}{lllll}0.415 & 0.422 & 0.428 & 0.435 & 0.442\end{array}$

Figure 8.11: Separation zone bounding streamline for $h_{b}=0.30$ case

The relation of deck height above the bed to the vertical contraction created by flow separation at the leading edge of the first I-beam is shown in Figure 8.12. The vertical contraction, which is the same as the thickness of the separation zone, $t$, increases nearly linearly with the height above the bed until the bridge deck is not fully submerged at $h_{b}=0.47$. 


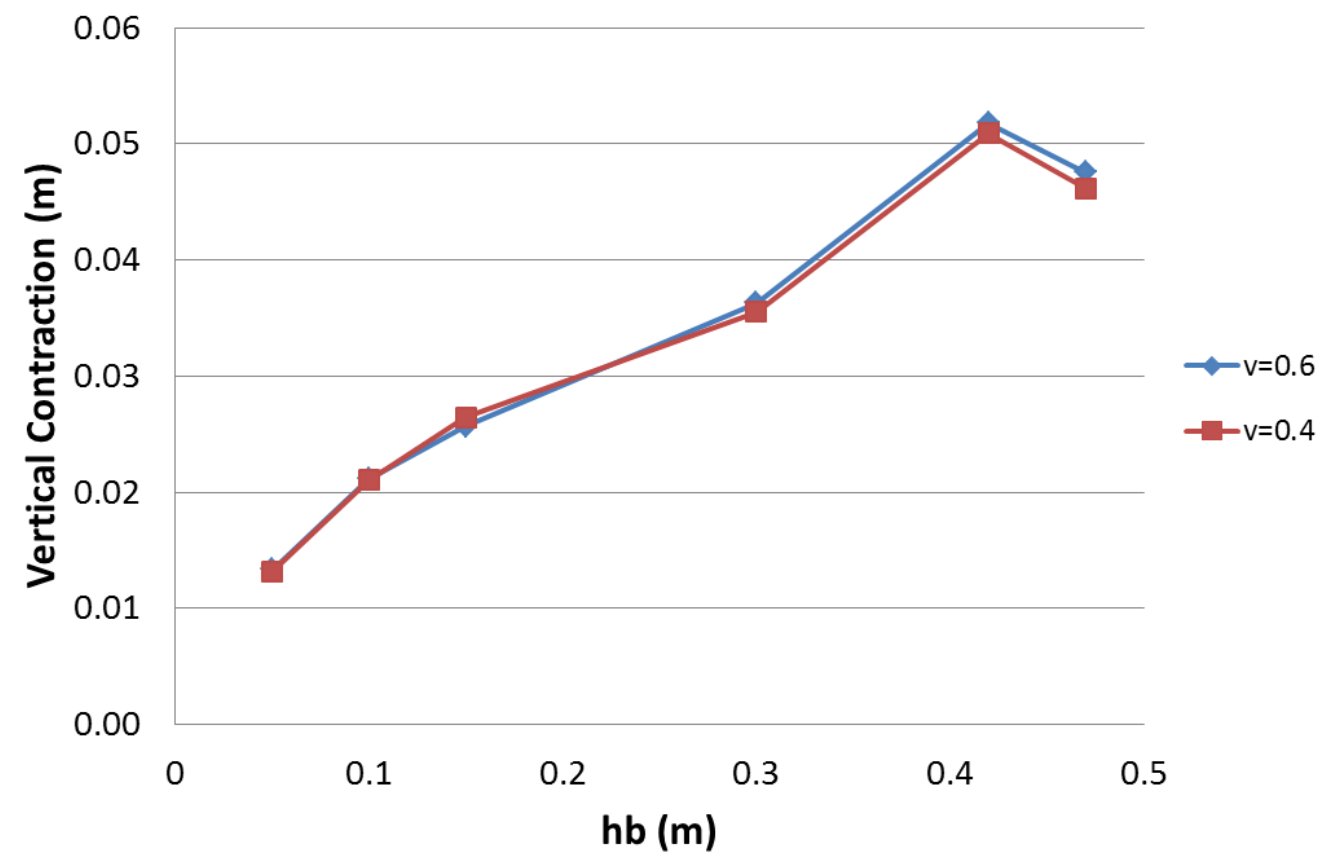

Figure 8.12: Vertical contraction of flow under bridge deck as a function of the height of the deck above the bed showing a dip at 0.47 when the deck is not fully submerged 


\subsubsection{Computational Modeling and Analysis of Flow through Large Culverts for Fish Passage}

Fish passage through culverts is an important component of road and stream crossing design. As water runoff volume increases, the flow often actively degrades waterways at culverts and may interrupt natural fish migration. Culverts are fixed structures that do not change with changing streams and may instead become barriers to fish movement. The most common physical characteristics that create barriers to fish passage include excessive water velocity, insufficient water depth, large outlet drop heights, turbulence within the culvert, and accumulation of sediment and debris. Major hydraulic criteria influencing fish passage are: flow rates during fish migration periods, fish species, roughness, and the length and slope of the culvert.

The objective of this work is to develop approaches to CFD modeling of culvert flows and to use the models to perform analysis to assess flow regions for fish passage under a variety of flow conditions. The flow conditions to be tested with CFD analysis are defined in the tables of a work plan from TFHRC [6]. The CFD models are being verified by comparing computational results with data from experiments conducted at TFHRC. A primary goal of CFD analysis of culverts for fish passage is to determine the local cross section velocities and flow distributions in corrugated culverts under varying flow conditions. In order to evaluate the ability of fish to traverse corrugated culverts, the local average velocity in vertical strips from the region adjacent to the culvert wall out to the centerline under low flow conditions will be determined.

A primary goal of the CFD analysis during this quarter has been to investigate methods to model gravel in the culvert. The test matrix in the TFHRC work plan [6] includes tests with the bed height at $15 \%$ and $30 \%$ of the culvert diameter. For these cases, the culvert bed material is coarse gravel with a mean diameter, $D_{50}=12 \mathrm{~mm}$. At this gravel size, the gravel bed boundary cannot be treated as a rough wall using wall functions because the centroid of the near wall computational cell must be at a position that is greater than the roughness height. For $12 \mathrm{~mm}$ gravel, the near wall mesh would be far too large for the analysis results to be mesh independent. Two options to model flow parallel to a porous gravel bed are (1) to treat the bed as a porous media, with a flat interface dividing the two flow zones, and (2) to mesh out the rough bed contour created by the top layer of gravel. The methods for generating various velocity averages over a cross section from the work plan [6] that may be applied in improved fish passage analysis are summarized in Section 1.3.2.1, and example results from a test case are presented.

\subsubsection{Velocity Curves for Culverts}

\subsection{Local Depth-Averaged Velocity Curve Development}

Flow velocity and flow depth are two important factors influencing fish activity in a culvert. Figure 8.13 illustrates the information that was measured in the flume and was calculated by the numerical modeling methods described below. At a given culvert cross-section, flow depth, and flow discharge, the local depth-averaged velocities $V_{1}, V_{2}, V_{3}$, etc. are measured at regular offsets from the culvert wall. Typically the local depth-averaged velocity will approach zero at the culvert wall and will be at a maximum near the center of the culvert. 

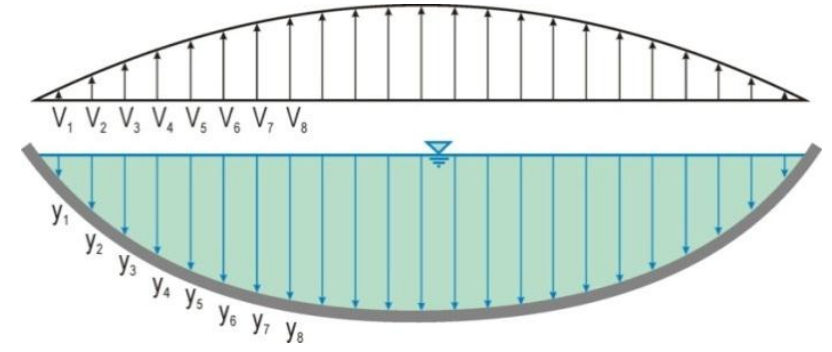

Figure 8.13: Variation of flow velocity and depth in a cross-section of a corrugated metal pipe

In the numerical modeling, the culvert cross-section is divided into evenly spaced strips, and then the grid's discharge and area in each strip are determined by integrating over the strip. The ratio of the integrated discharge and integrated area is the depth-averaged velocity. In this study, a trimmed cell mesh was used to generate an extremely high quality hexahedral-based mesh for the culvert geometry. This kind of mesh model gives a structured mesh across the culvert section that is well suited for computing strip averages because the grid can be built to align cell faces with strip boundaries. When these boundaries are not aligned with cell faces, velocity data in uniform vertical strips in the crosssection are generated by interpolating the original grid velocity data from STAR-CCM+. The exported data is then used to get the depth averaged velocity distribution over the strips by averaging the velocities falling in each strip with MATLAB. The results are shown in Figure 8.14. 

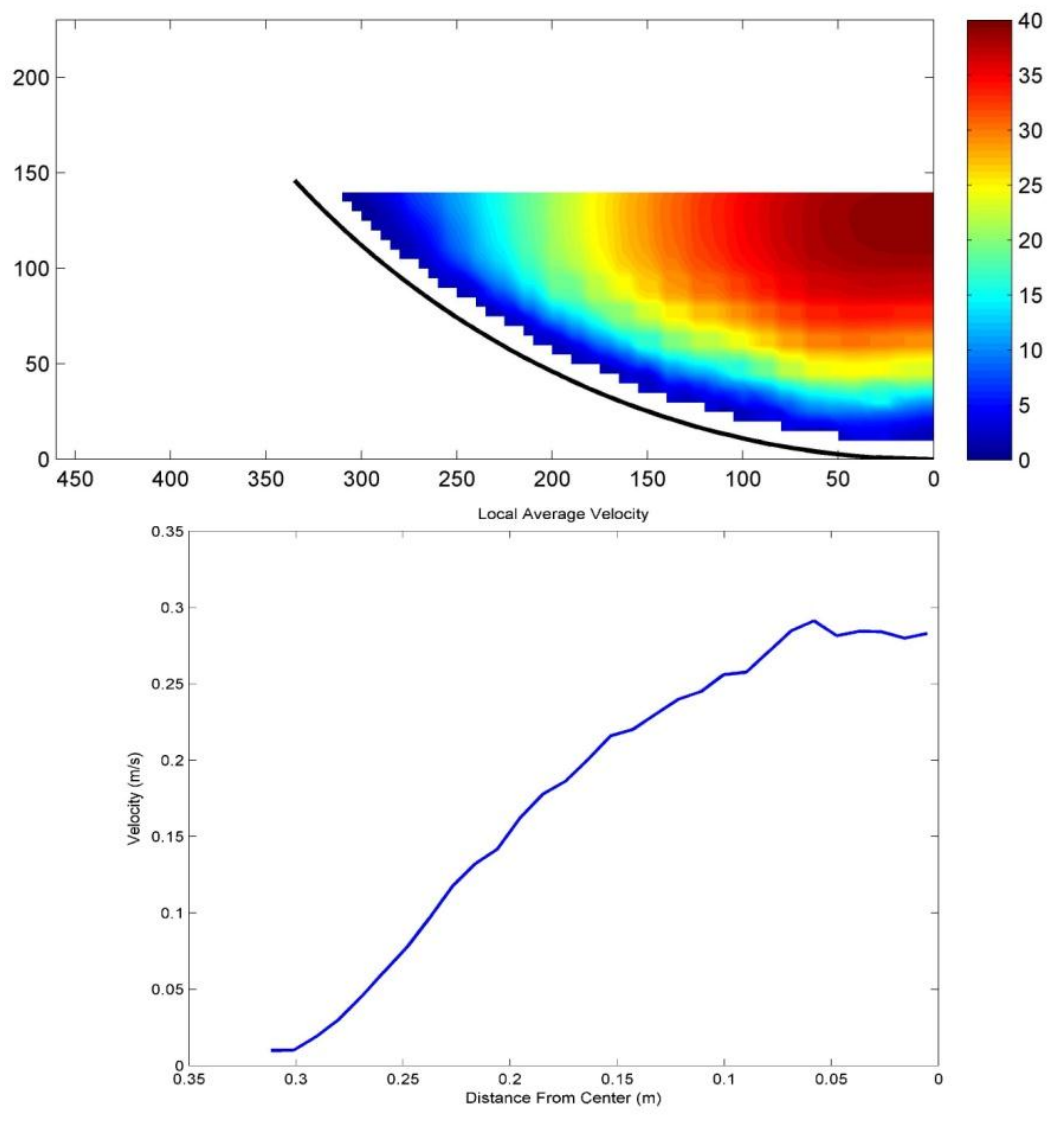

Figure 8.14: Depth-averaged velocity curve development in a cross-section of 6 inch water depth with symmetry boundary

In a similar manner, the local flow depths y1, y2, y3 can be measured at the same offsets from the wall that are used for the velocity readings. These depths can be related to the maximum flow depth $y_{\max }$ through the culvert geometry. The relationship between the local depth-averaged velocity $V$ and the local depth y of the flow at any point in the culvert cross-section is the important information needed for the Aquatic Organism Passage design of the culvert. The fish design criteria, can be provided by the appropriate environmental agency as noted in the discussion of Tables $1 A$ and $B$ in the work plan [6]. The next step is to predict velocity distribution and depth variations through culverts under various conditions, including flow depths, inlet velocities, bed elevations, and roughness. A path through a culvert suitable for fish passage can be defined for the given conditions from strip depth average velocities. The fish path in the culvert is shown in Figure 8.15. 

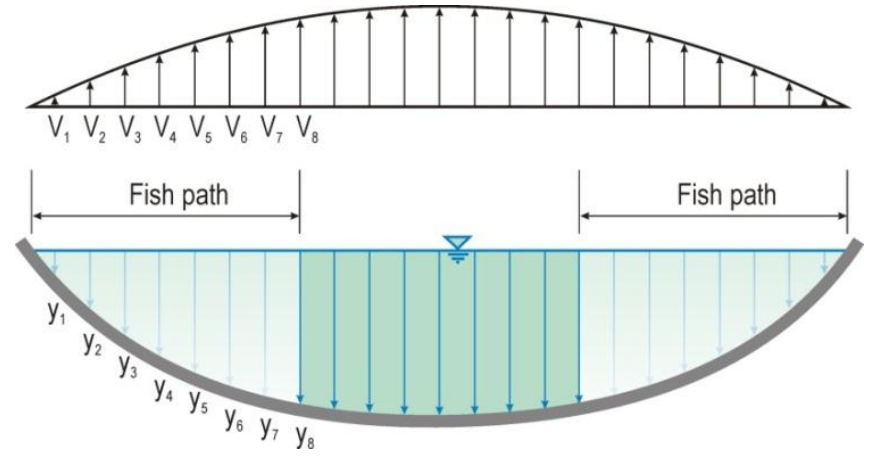

Figure 8.15: Flow path for the selected fish design criteria of velocity and depth

In Figure 8.15, one end of the path is defined by the culvert wall. The other end of the path, towards the center of the culvert, is defined by the point where the local depth-averaged flow velocity $V$ is equal to the maximum fish swimming velocity, $V_{F}$, as defined by the appropriate standard. In this illustration example $V_{8}=V_{F}$. Note that another limit to the fish path can be the water depth when the depth in a strip is less than that required for larger species of fish, such as trout or salmon. 


\subsection{Average Velocity Curve Development}

The area (A) and volume flow rate (Q) of the flow within the limits of the fish path also can be computed, and the average velocity of the flow within the fish path can be computed as illustrated below in Figure 8.16 and Figure 8.17.

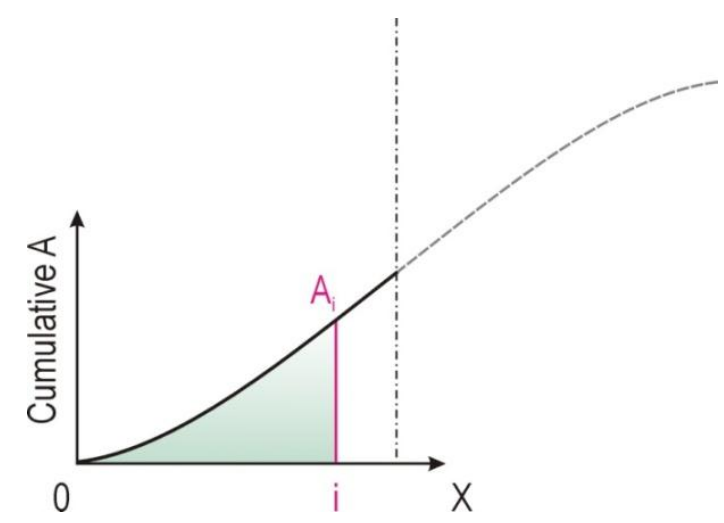

Figure 8.16: Cumulative area from the wall to the centerline

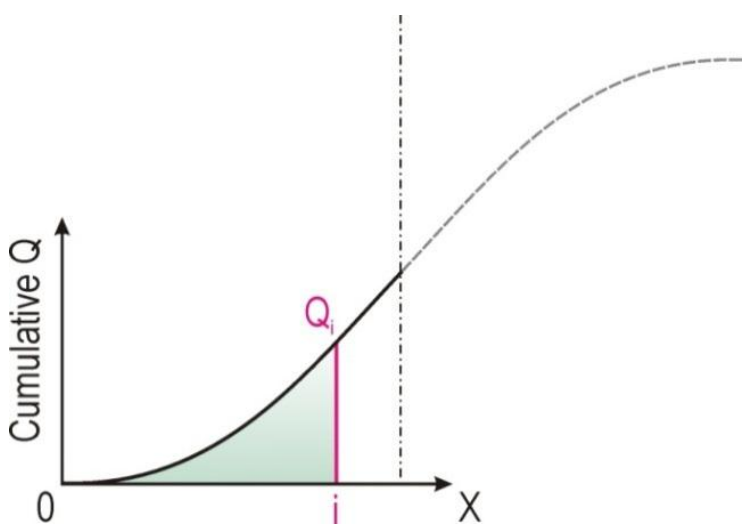

Figure 8.17: Cumulative discharge from the wall to the centerline

Equation 8.1 can be used to compute the average velocity between the outer culvert wall and position i:

$$
\left(V_{A V G}\right)_{i}=\frac{Q_{i}}{A_{i}}
$$

For the flow condition illustrated in Figure 8.14, the cumulative discharge (Q), cumulative area (A), and the corresponding average velocity can be computed with MATLAB, as shown in Figure 8.18 through Figure 8.20. 


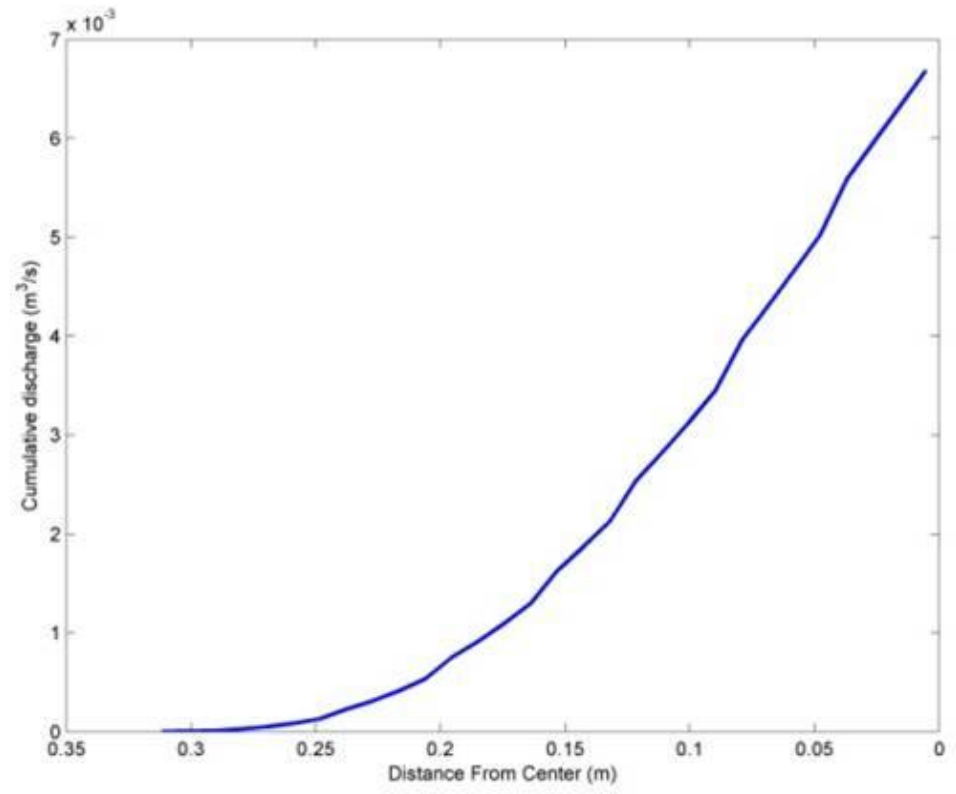

Figure 8.18: Cumulative discharge from the wall to the centerline in a cross-section of 6 inch water depth with symmetry boundary

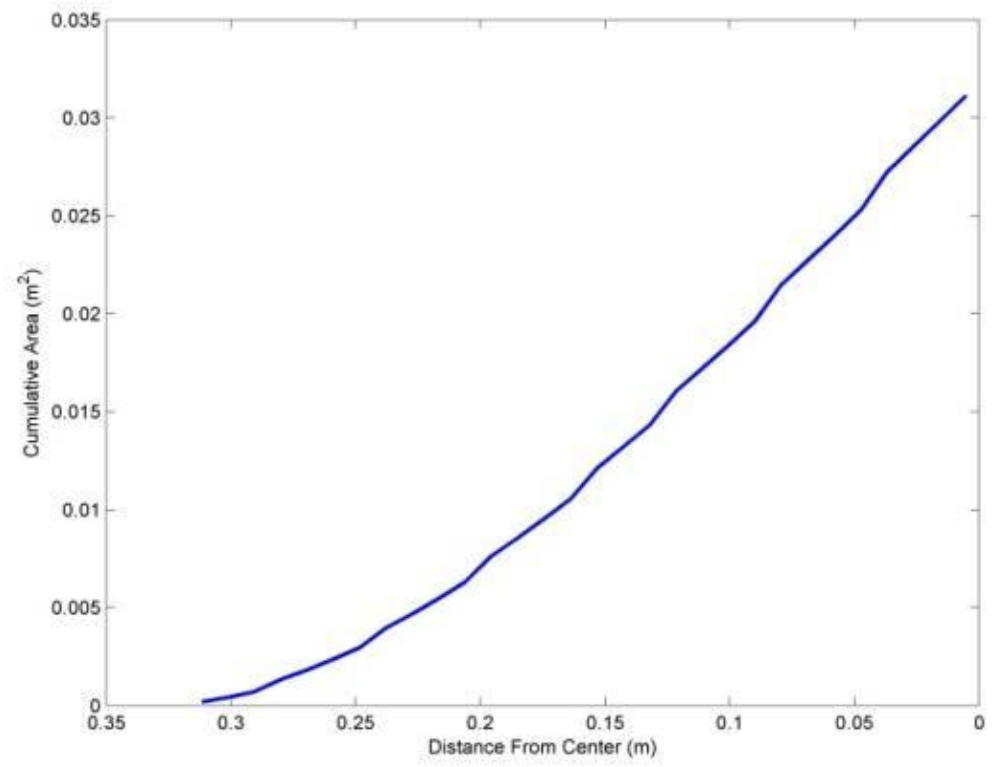

Figure 8.19: Cumulative area from the wall to the centerline in a cross-section of 6 inch water depth with symmetry boundary 


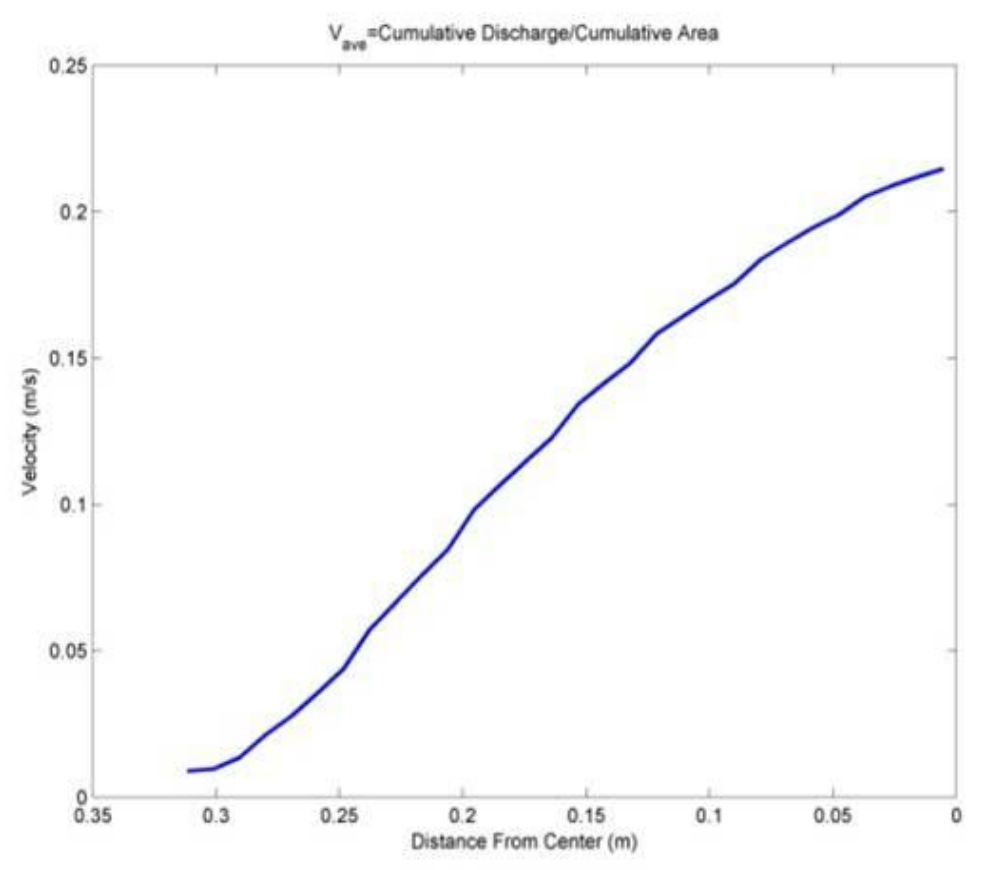

Figure 8.20: average velocity curve in a cross-section of 6 inch water depth with symmetry boundary

\subsubsection{Porous Media Modeling}

For the larger size gravel beds in culverts, the gravel bed cannot be modeled as a rough wall with wall functions used to determine the wall shear stress because the near wall cell height would be a significant fraction of the water depth, making the grid too coarse to obtain an accurate solution. Two alternatives to modeling the effects of the presence of a gravel bed are (1) to include the large scale roughness in the geometry and mesh the irregular surface of the bed, and (2) to model the bed as a porous media. Porous media models in CFD software usually treat the porous media part of the domain as a distributed volumetric flow resistance. Porous media models capture the overall effects of the presence of the porous region on a flow without resolving the small scale details of the flow in the interstitial spaces of the media. Modeling the effects of a large diameter gravel bed in a culvert on the main flow above the bed appears to be a good fit into the class of problems that can be handled with the porous media model.

Some difficulties arise as a consequence of using short sections of culvert with cyclic boundary conditions to determine the cross section velocity for fully developed flow conditions. When a porous media is present in the bed subdomain, the cyclic boundary conditions cannot be applied to the porous subdomain. This restriction means that a section of culvert modeled with cyclic boundary conditions and porous media for the bed is equivalent to having a gravel bed with plates blocking flow in the porous media between sections. The effect of this limitation is expected to be small. The work to set up the porous media models was completed, initial tests were run, and the results are presented in the following sections. A comparison of the previously used short section and a section five times as long, which allows for more induced flow in the porous media, was also done to see if there is a significant difference. 


\subsection{Porous Media Parameters}

The TFHRC work plan for the culvert studies [6] has cases with large gravel sizes of $12 \mathrm{~mm}$ and $24 \mathrm{~mm}$ that will be modeled using a porous media subdomain. The parameters characterizing the porous media in the model are the porosity, permeability, and the beta factor coefficient of the Forchheimer inertial momentum sink term in the porous media momentum equation. In absence of experimental or other material property information for the gravel in the bed, the gravel is taken to be a packed bed of spheres with diameter equal the mean gravel diameter. The porosity of packed beds of spheres can vary depending on how densely they are packed. The porosity was taken to be 0.40 . This value corresponds to a midrange packing that would be typical of poured random packing.

For packed beds, the STAR-CCM+ User Guide [7] gives the permeability, $k_{p}$, as:

$$
\frac{1}{k_{p}}=\frac{150(1-\chi)^{2}}{\chi^{3} D_{p}{ }^{2}}
$$

where and $D_{p}$ is the mean diameter of particles in the porous medium, and $\chi$ is the porosity, The inertial term beta factor is given as:

$$
\beta=\frac{1.75(1-\chi)}{\chi^{3} D_{p}}
$$

where $\rho$ is the fluid density. The coefficients $P_{v}$ and $P_{i}$, of the viscous and inertial momentum sink terms for packed beds in the STAR-CCM+ porous media model are user inputs that define the model parameters and they contain the viscosity, $\mu$, and density, $\rho$, of the fluid (water):

$$
P_{v}=\frac{150 \mu(1-\chi)^{2}}{\chi^{3} D_{p}^{2}}
$$

And

$$
P_{i}=\frac{1.75 \rho(1-\chi)}{\chi^{3} D_{p}}
$$

\subsection{Implementation of Porous Media in STAR-CCM+}

CD-adapco's STAR-CCM+ software provides a mechanism by which field functions can be used to modify the physics models of the flow or define parameters in physics models. The specification of porous inertial resistance and porous viscous resistance along with the other input conditions and values are enabled for a porous region to specify the coefficients of flow resistance. These coefficients are used to calculate the porous media sink terms in the momentum equation, as detailed in the formulation. 


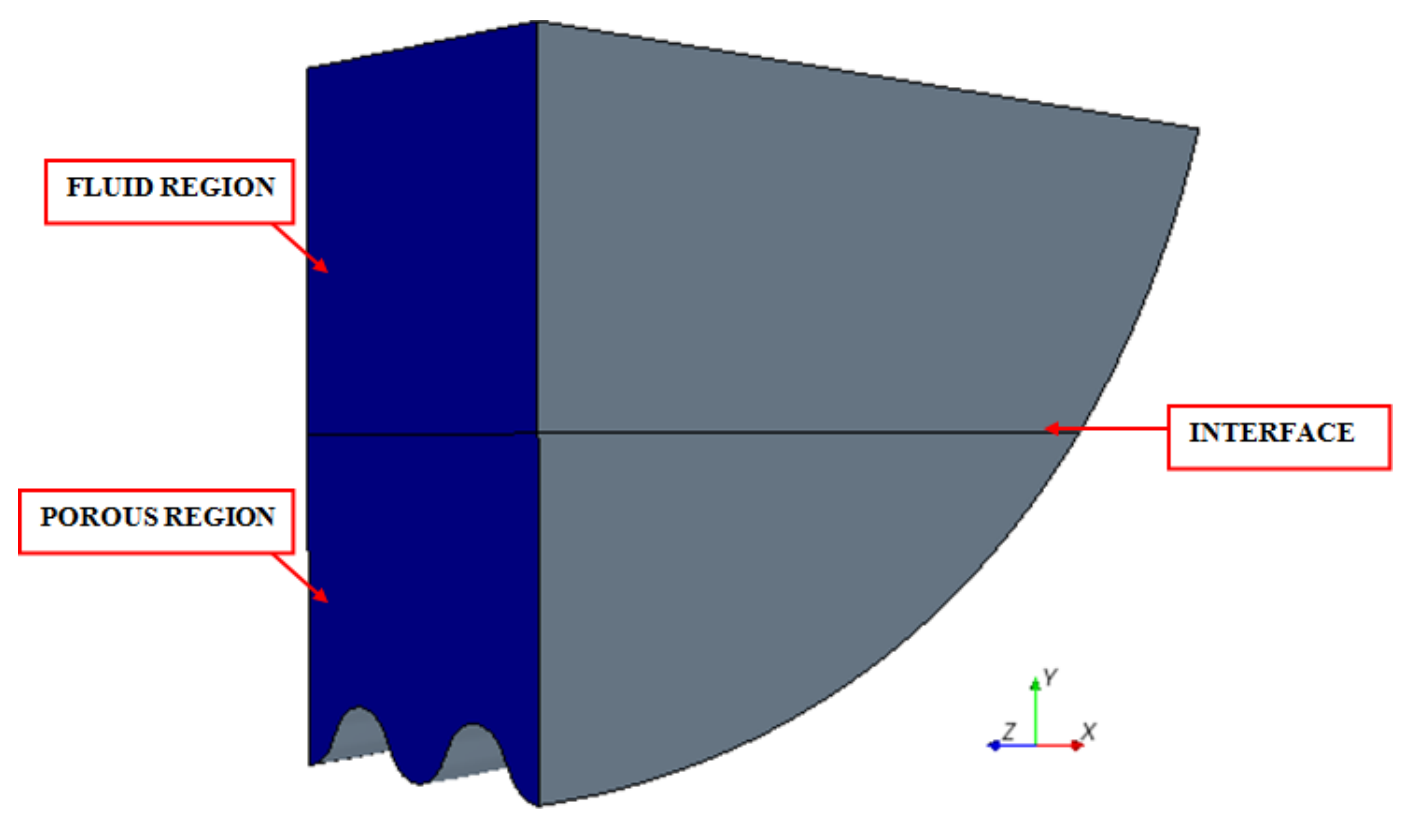

Figure 8.21 CAD model representing the computational domain used for porous media modeling

\subsection{Description of the Physical Model}

In this study, a simulation model was developed using the commercial CFD software STAR-CCM+. A small section of the culvert barrel was modeled with cyclic boundaries at the inlet and outlet sections of the computational domain in the fluid region as in the previous studies for flow through a culvert with no gravel. For simulating the flow within the porous region, the ends of the domain with the cyclic boundaries are treated as walls in the lower porous region because the cyclic boundary conditions cannot be applied when there are multiple types of inlet and outlet boundaries to be coupled. A 36 inch diameter culvert with corrugation size 3 inches by 1 inch has been used for this study. A flow depth of 6 and 9 inches for a flow velocity of 0.71 feet/second is considered for this study.

\subsection{Boundary Conditions for the Porous and the Fluid Regions}

The physical model consists of two regions namely the fluid region, which is the focus of the study and the porous region (gravel section) which is being simulated using the porous media approach. The following are the boundary conditions used for the fluid region. 
Table 8.2 Boundary conditions

\begin{tabular}{|c|c|c|}
\hline Boundary & Name & Type \\
\hline Face at minimum $x$ value & Inlet & Cyclic boundary condition \\
\hline Face at maximum $x$ value & Outlet & Cyclic boundary condition \\
\hline Water surface & Top & Symmetry plane \\
\hline Centerline & Center & Symmetry plane \\
\hline all other surfaces & Barrel & No-slip wall \\
\hline
\end{tabular}

For the porous region, the boundary at the centerline is taken to be a symmetry plane and all other surfaces are considered as no-slip wall. In Figure 8.21, the interface between the fluid and the porous region, which is taken to be an in-place boundary, is shown.

\subsection{Meshing Methodology}

A strategic meshing technique has been adopted to mesh the model to get good CFD results. It is very important to have an optimum number of cells in the mesh to have a good flow resolution. As a part of the meshing strategy, a volumetric control (annulus ring) has been created along the corrugated section. The mesh is refined along the volumetric control with respect to the other regions of the computational domain. The refinement of the mesh is defined by specifying a reduction of mesh size for volume within an annulus intersecting the model as shown in Figure 8.22. The volumetric control body intersecting the corrugated section provides a means to refine the mesh in the corrugated region of interest. The refined mesh enables better resolution of the flow field with recirculation zones at the troughs between the corrugations. Also a block has been created at the interface of the fluid and the porous regions where the mesh is further refined. The purpose of creating a block for mesh refinement is the same as that of creating an annulus ring around the corrugated section. Meshing also includes a prism layer consisting of orthogonal prismatic cells running parallel to the wall boundaries, which constitutes a boundary mesh that is good for the application of wall functions to compute the shear stress at the wall boundaries. 

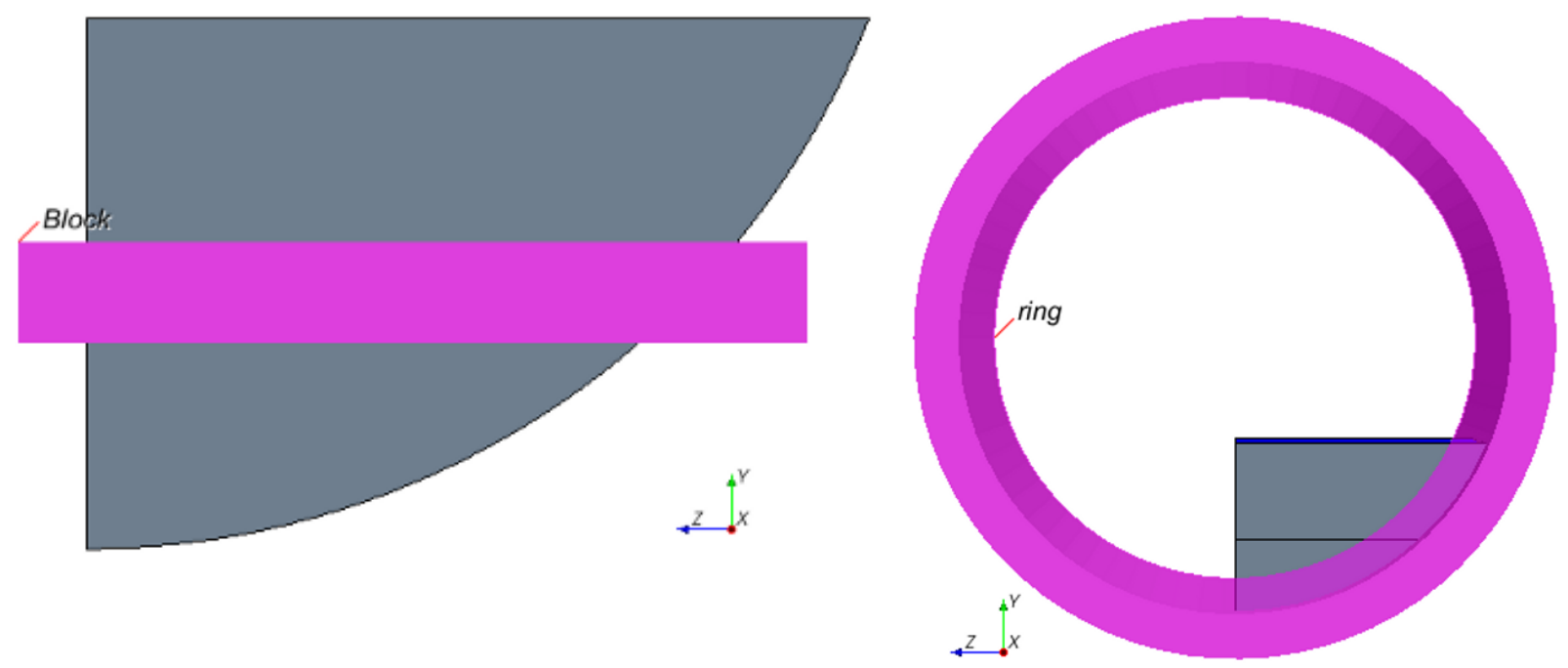

Figure 8.22 Volumetric controls created around regions of major interest for mesh refinement

From the mesh refinement studies, done to find the optimum base size of the mesh for a 36 inch diameter of the culvert with corrugation size 3 inches by 1, for a flow depth of 6 inches and 9 inches, it was found that a base size of $5 \mathrm{~mm}$ and $67 \%$ refinement in the volumetric controls is a good size for the mesh which gives mesh independent simulation results with adequately fast run times. The same base size of the mesh and refinement along the volumetric controls has been used for the present study.
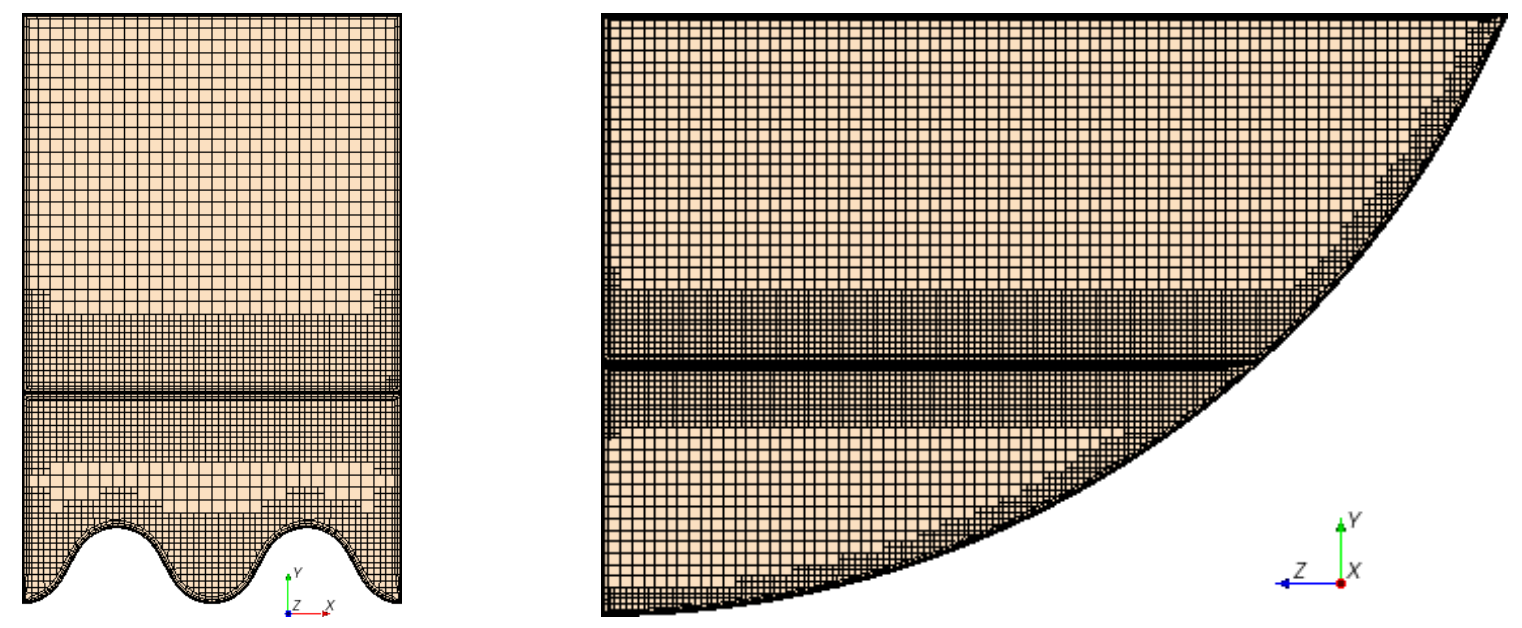

Figure 8.23 Cross sectional view of the mesh scenes

\subsection{Comparison of the Reduced Section of a Culvert with an Increased Section}

A reduced section with cyclic boundary conditions has been used for all CFD tests. Working with a reduced section is computationally fast, allowing a couple of tests to be completed in one day. In order to check the reliability of the results with blocking walls in the porous section at the cyclic boundaries of 
the culvert, a comparison study has been conducted to check for significant differences in both the fluid and the porous regions when the modeled section length is significantly increased allowing more opportunity for induced flow to develop in the upper part of the porous media when compared to a reduced section of only two corrugation crests.

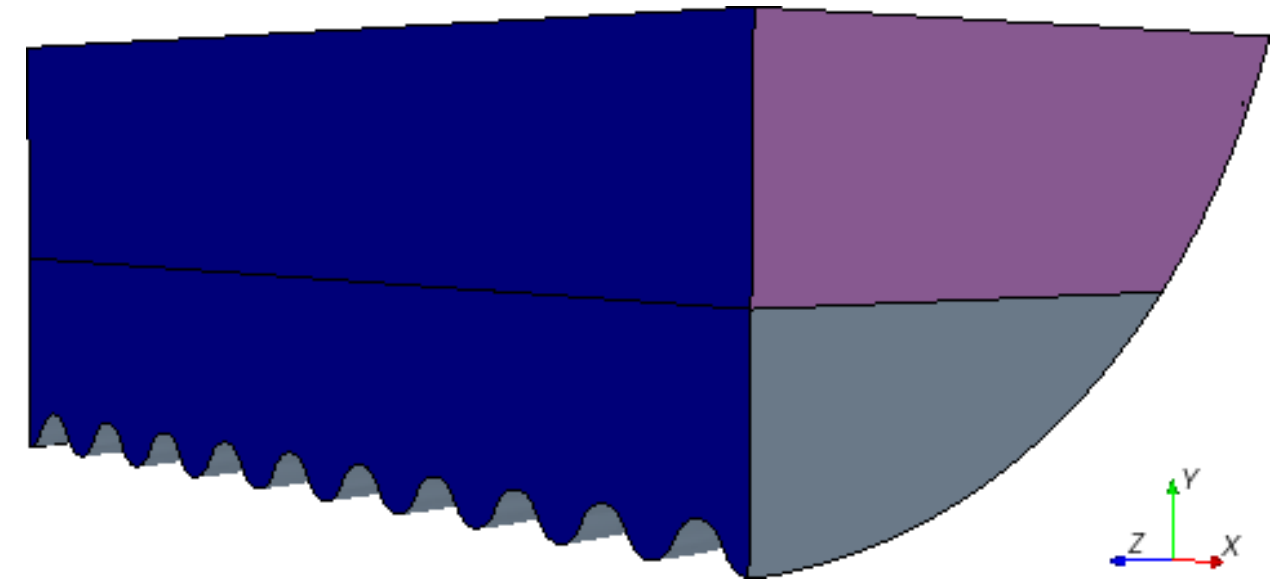

Figure 8.24 Increased section of the culvert (nearly five times bigger than the reduced section) used for porous media modeling

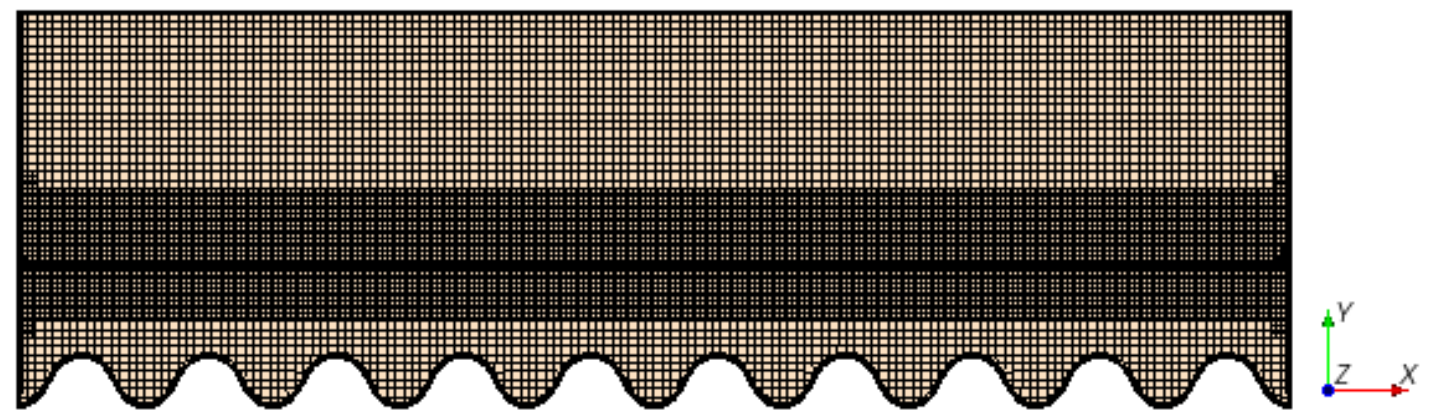

Figure 8.25 Cross sectional view of mesh scene along on a plane taken along the length of the increased section

For this comparison study, an increased section has been created. The increased section is 5 times longer than the reduced section. The CFD run corresponding to the increased section has the same mesh and flow parameters as that of the reduced section. After mesh generation, it was found that the volume mesh for the reduced section contains about 554176 cells and the volume mesh for the increased section contains about 2468562 cells. The results of each of these tests are presented in the following sections.

\subsection{Results and Discussion}

Line probes have been created along the flow section in the STAR-CCM+ software. In this particular case line probes have been created at a trough and a crest which are the regions of major interest. Each of the line probes created has 30 points on the line. The value of the velocity magnitude of the flow is extracted at each particular point. Velocity profiles have been plotted using the line probes at a trough 
and a crest for both the reduced barrel and the increased sections. By taking a close look at the velocity profiles, it is possible to better analyze the nature of the flow.
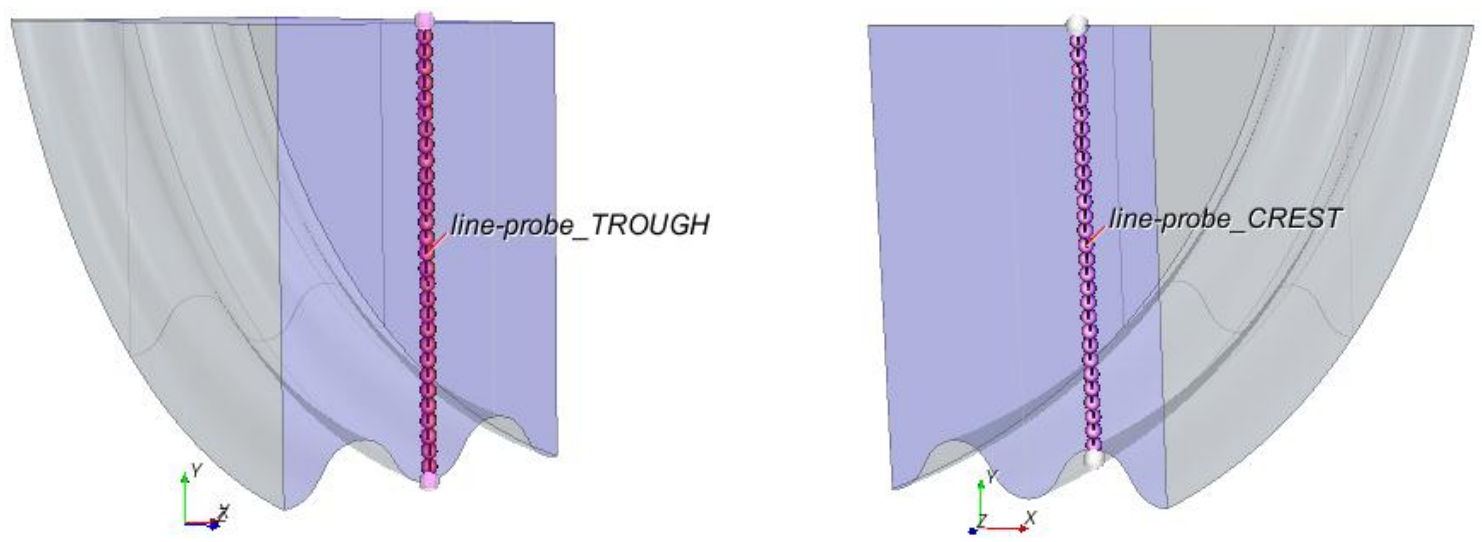

Figure 8.26 Image depicting the line probes created at a trough and a crest for a reduced section

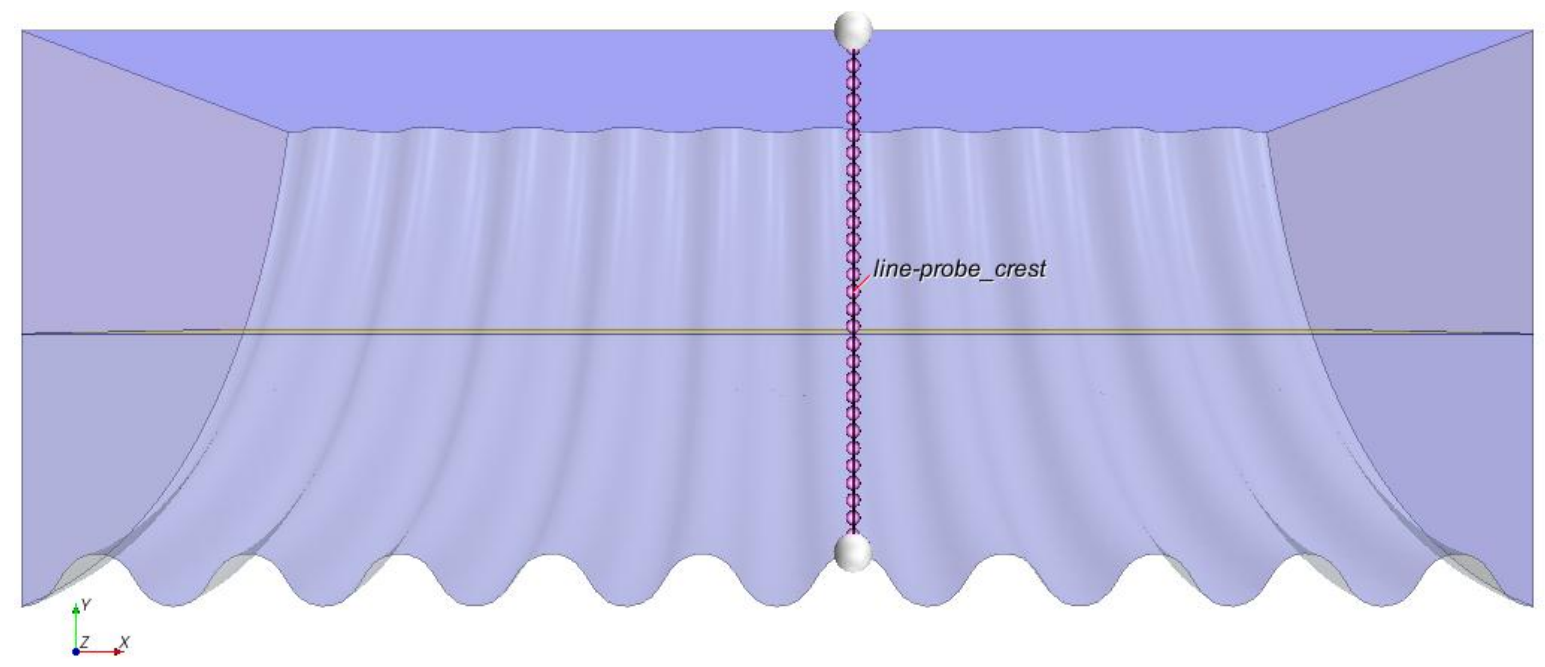

Figure 8.27 Image depicting the line probe created at a crest for an increased section

In Figure 8.28, the x-axis of the plot represents velocity and the $y$-axis represents the position of the line probe at a trough in the vertical direction. The minimum unit on the $y$-axis is $0.15 \mathrm{~m}$ and the maximum unit is $0.45 \mathrm{~m}$. The y coordinate of the boundary representing the water surface (namely the top of the reduced culvert section in the CFD study) is at $0.17 \mathrm{~m}$ and the y coordinate of the boundary representing the bottom of the culvert at the wall is at $0.4572 \mathrm{~m}$. The same CAD model has been used for all the CFD simulations with the co-ordinates of the reduced symmetric barrel section considered from a trough to a trough as mentioned above. The top surface of the culvert is simulated as a symmetry plane as mentioned previously which represents an imaginary plane of symmetry in the simulation. It implicates an infinitely spread region modeled as if in its entirety. The bottom of the culvert is simulated as a wall 
with a no slip condition. When velocity is plotted against position, the velocity at the wall is zero, the first point plotted is the velocity in the cell next to the wall and increases with distance from the wall.

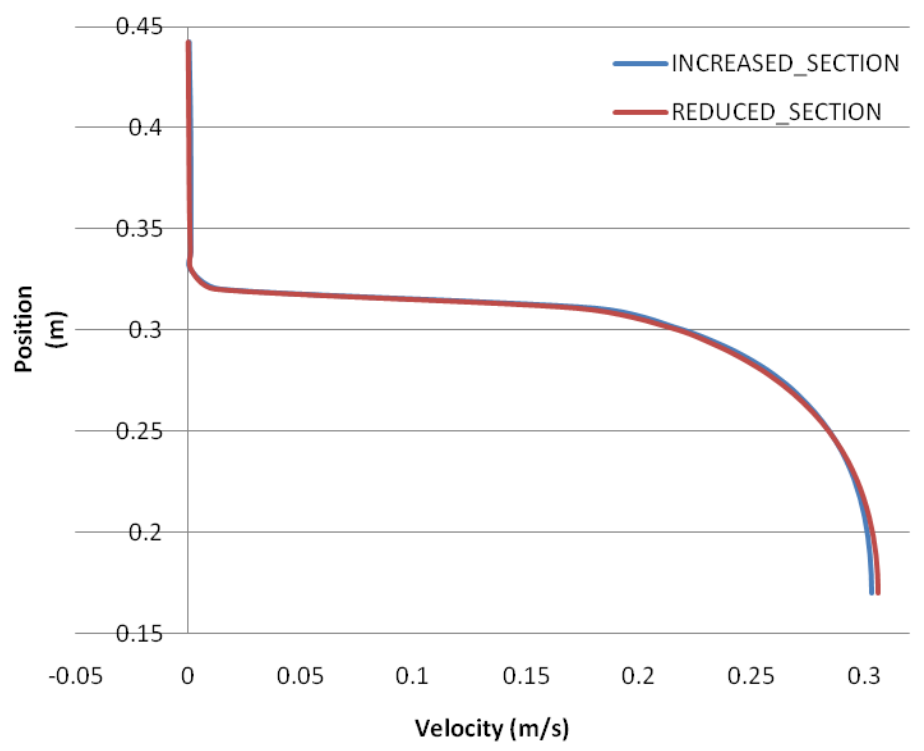

Figure 8.28 Velocity profiles for both reduced and increased sections at a crest

In Figure 8.28, the velocity and the position corresponding to the line probe at a crest are plotted on the $x$ and $y$ axis respectively. Figure 8.29 shows a similar comparison at a trough. The velocity in the porous region is very close to zero. The velocity profile in the fluid region is close to that of one with a wall boundary. The profiles for reduced and long barrel sections are nearly identical, and therefore the shorter section appears to be reasonable to use for determining the cross section velocity distribution above the porous media in parametric tests for different conditions and culvert geometry parameters with a gravel bed. 


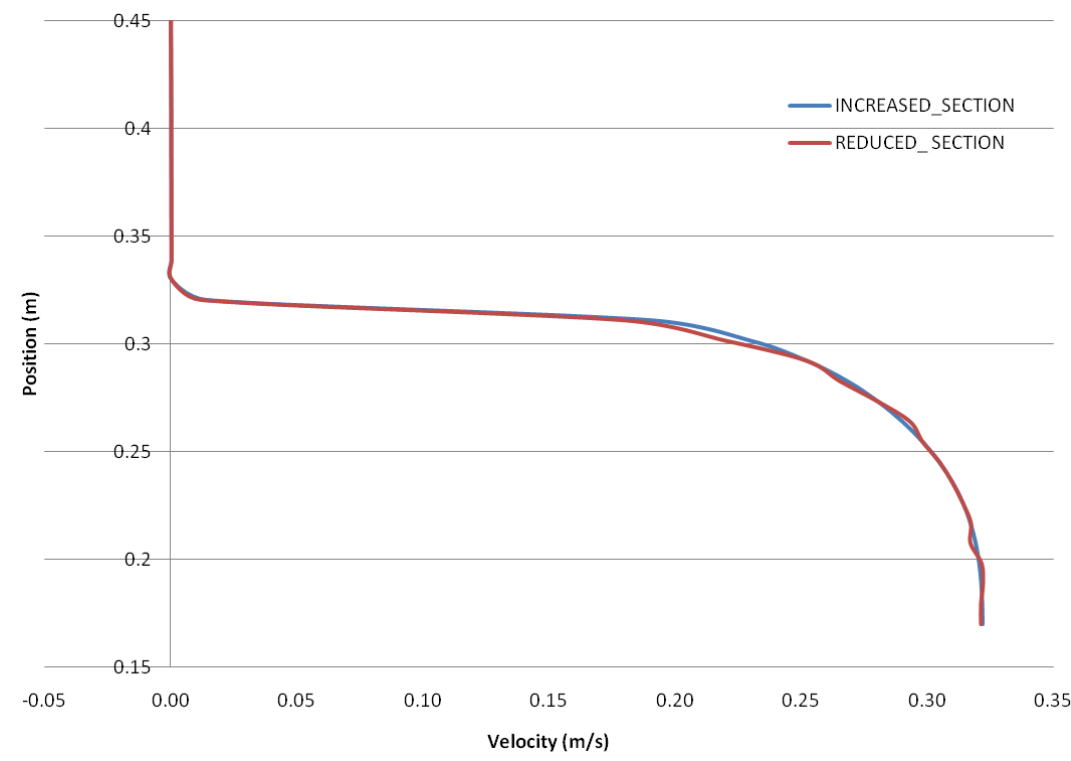

Figure 8.29 Velocity profiles for both reduced and increased sections at a trough

Uniform strips were created on the plane section at a crest in the test case. Figure 8.31 shows the odd numbered strips created on the plane section at a crest in the fluid region. This procedure is carried out by creating multiple "Thresholds" of $1 \mathrm{~cm}$ width along the plane section that align with grid cell faces. They are aligned with cell faces to avoid interpolation error and obtain the mean strip averaged velocity based on cell centroid values. After the thresholds are created, there is a "Report" feature available in STAR-CCM+ which calculates the surface averaged velocity over a vertical strip object. 


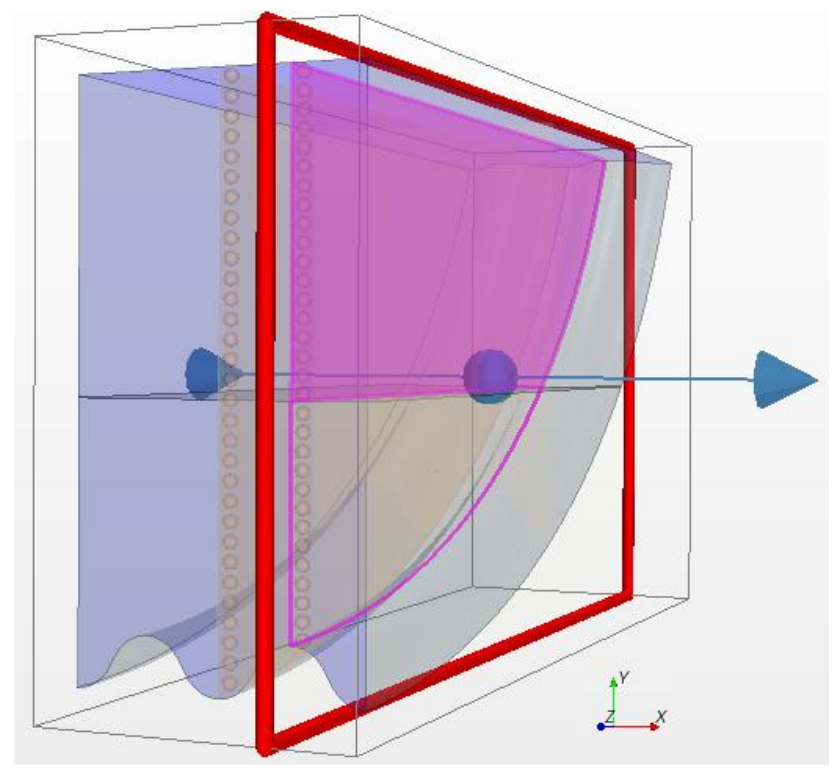

Figure 8.30 Cross sectional plane created at a crest in the porous region for a reduced section

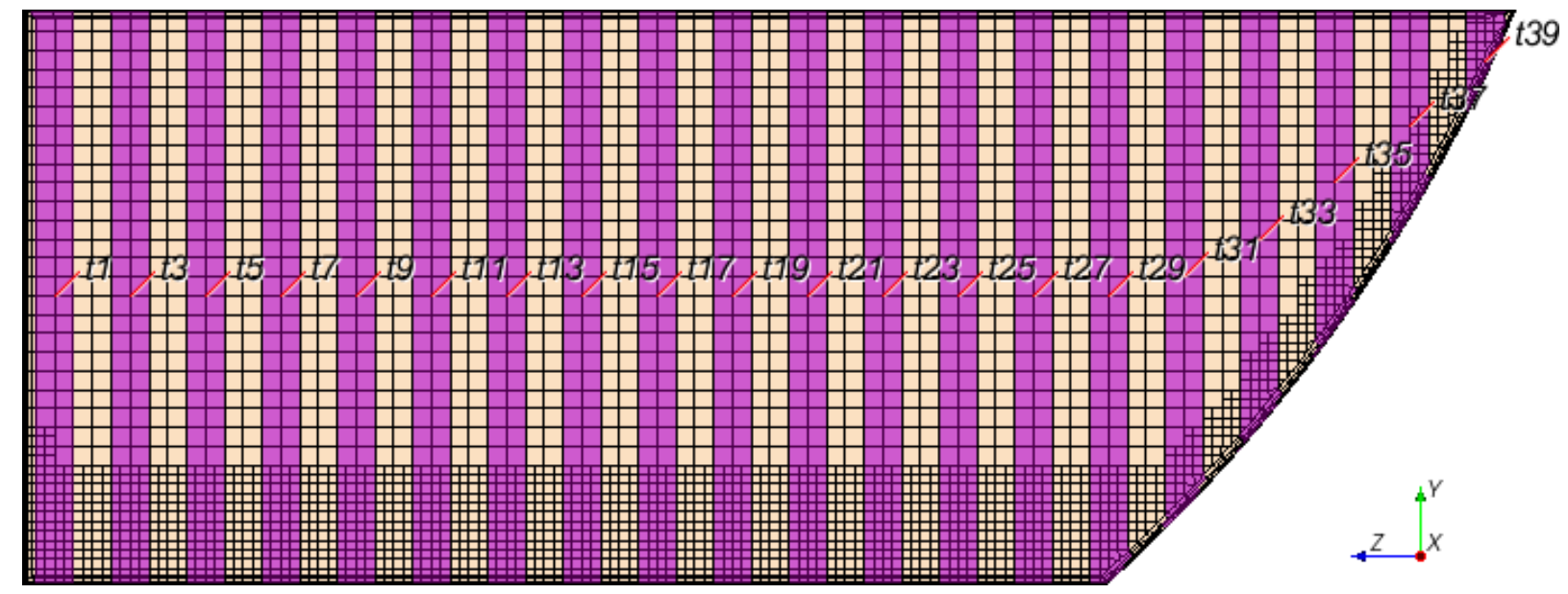

Figure 8.31 Representation of odd numbered Uniform strips of $1 \mathrm{~cm}$ width created along the fluid section

In Figure 8.32 a plot of surface-averaged velocities of the strips on the plane section at a crest is plotted across the width of a cross section, where the centerline is at zero. The $y$ axis of the plot indicates the position of a strip and the $x$ axis of the plot indicates the strip-averaged velocity. 


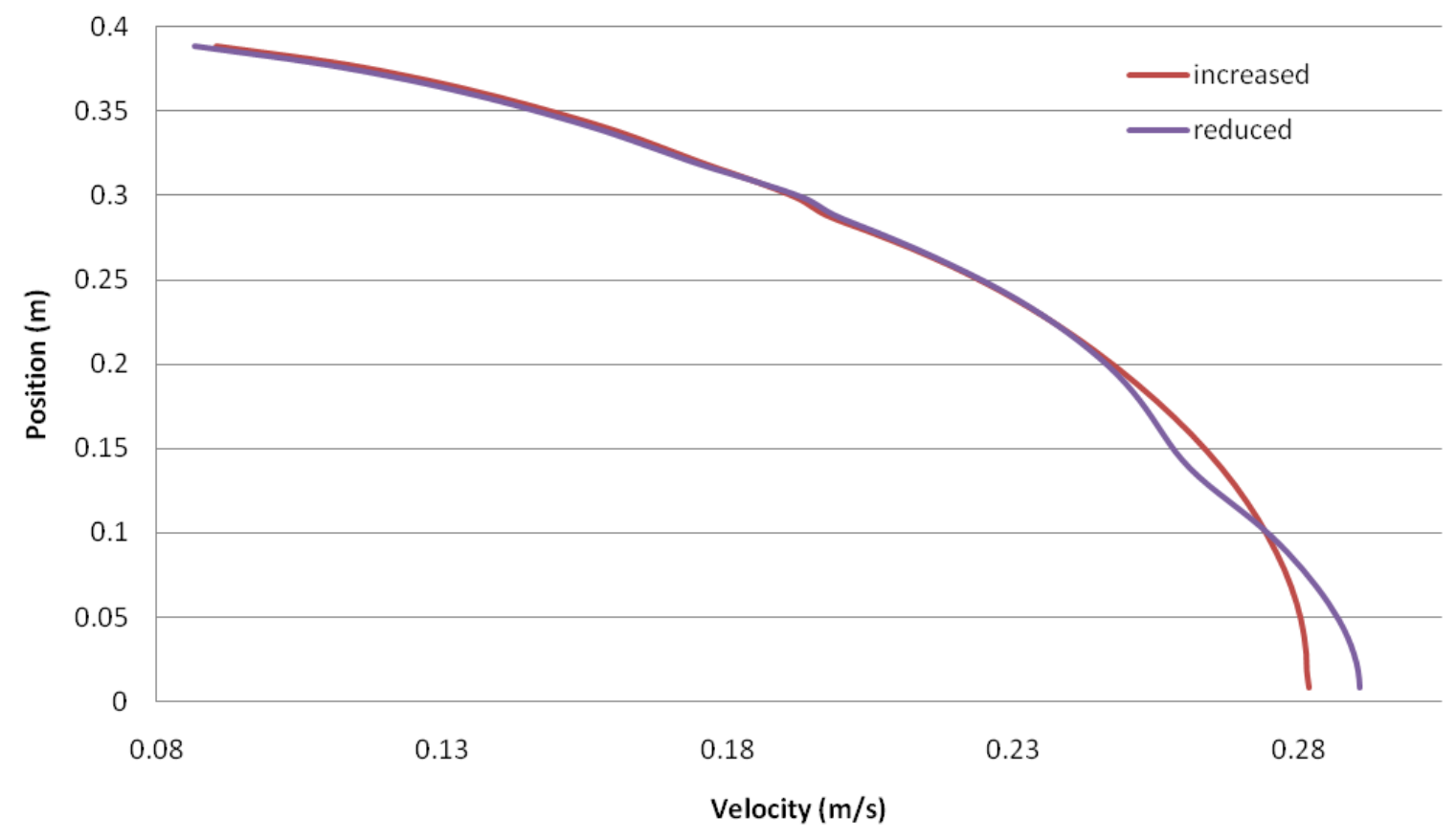

Figure 8.32 Surface-averaged velocity variation along the uniform strips plotted using "Threholds"

The origin on the $y$-axis corresponds to the centerline of the barrel section and the maximum point on the $y$-axis corresponds to the corrugated wall. It can be seen that for both the increased and reduced section the maximum velocities differ only slightly. The percentage difference in the results for the increased section and the reduced section is small, 3.7\% and considered to be adequate for engineering analysis.

Figure 8.33 shows the velocity distribution over the culvert cross section with a porous media gravel bed with velocity near zero. The effect of the porous bed on the flow above is as expected: it acts as a walllike flow resistance bringing the velocity down near zero at the interface between the free flow region and the porous media. There is a small amount of waviness in the contours approximately midway between the centerline and the outer culver wall that may merit some further investigation. A possible fish passage zone pattern is apparent as a lower velocity yellow region away from the centerline. 

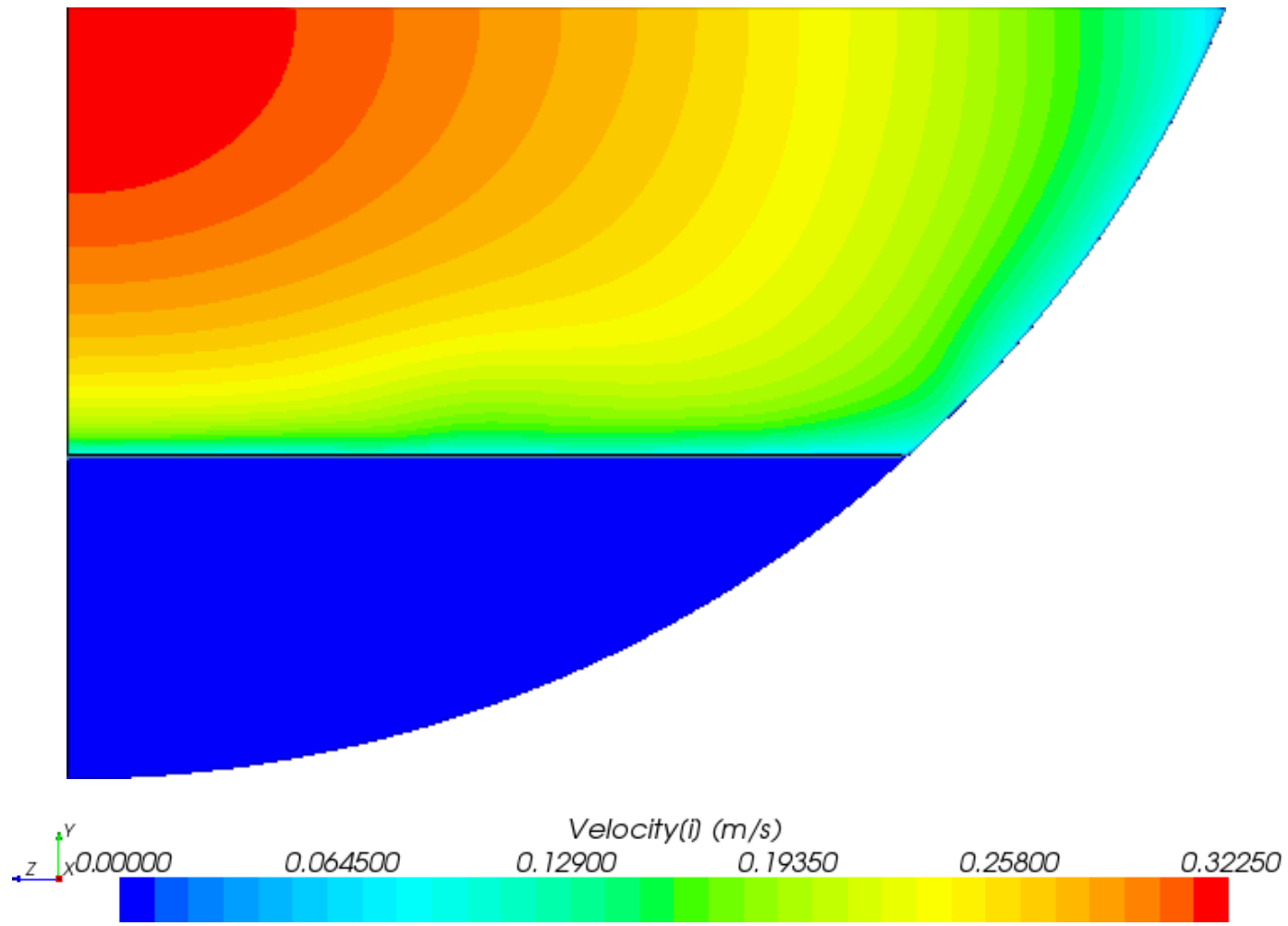

Figure 8.33: Velocity distribution over cross section at a crest showing the variation above the porous media gravel bed

\subsubsection{References}

1. Matt Blank, Joel Cahoon, Tom McMahon, "Advanced studies of fish passage through culverts: 1-D and 3-D hydraulic modeling of velocity, fish expenditure and a new barrier assesment method," Department of Civil Engineering and Ecology, Montana State University, October, 2008 .

2. Marian Muste, Hao-Che Ho, Daniel Mehl, "Insights into the origin \& characteristic of the sedimentation process at multi barrel culverts in lowa", Final Report, IHRB, TR-596, June, 2010.

3. Liaqat A. Khan, Elizabeth W.Roy, and Mizan Rashid, "CFD modelling of Forebay hydrodyamics created by a floating juvenile fish collection facility at the upper bank river dam", Washington, 2008.

4. Angela Gardner, "Fish Passage Through Road Culverts" MS Thesis, North Carolina State University, 2006.

5. Vishnu Vardhan Reddy Pati, "CFD modeling and analysis of flow through culverts", MS Thesis, Northern Illinois University, 2010.

6. Kornel Kerenyi, "Final Draft, Fish Passage in Large Culverts with Low Flow Proposed Tests" unpublished TFHRC experimental and CFD analysis of culvert flow for fish passage work plan, 2011.

7. CD-adapco, User Guide STAR-CCM+ Version 6.04.014, 2011 


\subsubsection{Initial Modeling of the Wind Tunnel Laboratory at TFHRC}

\subsubsection{Model Development}

A study of the TFHRC wind tunnel CFD modeling was initiated in this quarter. It aims to provide TFHRC researchers with the answers to the following questions:

- What is the overall quality of the flow in the testing section of the tunnel?

- What is the influence of the room walls' proximity on the air flow in the room?

- What is the influence of the room setup (including layout of the furniture and the equipment) on the flow quality?

A CAD data file containing the detailed geometry of the wind tunnel was initially provided by TFHRC (see Figure 8.34). It was importable to STAR-CCM+, although the geometry of the fan and fan inlets were overly simplified and required modifications.

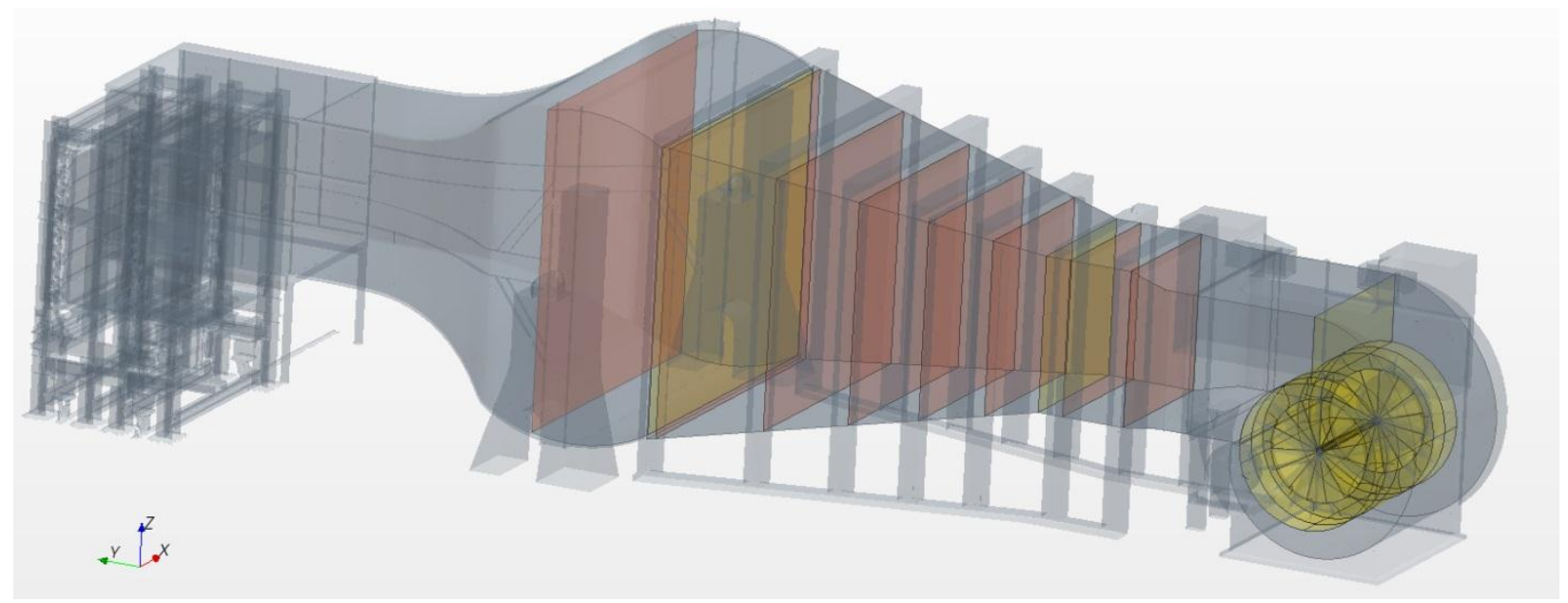

Figure 8.34: CAD model imported to STAR-CCM+

Figure 8.35 and Figure 8.36 show changes that were made to these parts of the model. Although the fan inlets closely resemble the real shape now, the fan is still simplified and in future may need additional improvements. Nevertheless, for the purpose of this study, it is believed that the actual geometry of the fan and inlets should provide reasonably good results for engineering assessment of the effects of room wall and furniture on wind tunnel air flow. 

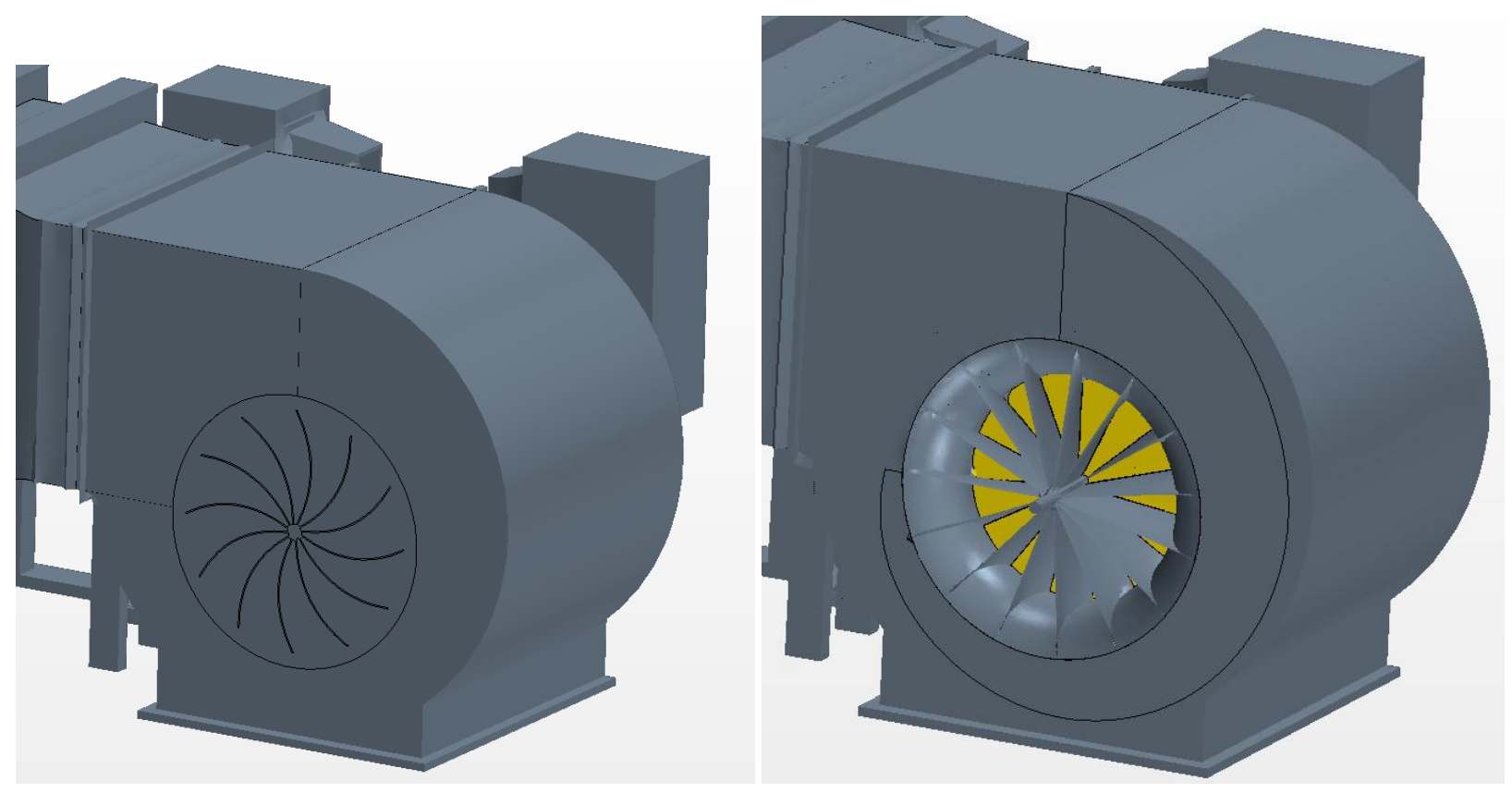

Figure 8.35: Geometry of the fan inlets: initial (left) and updated (right)
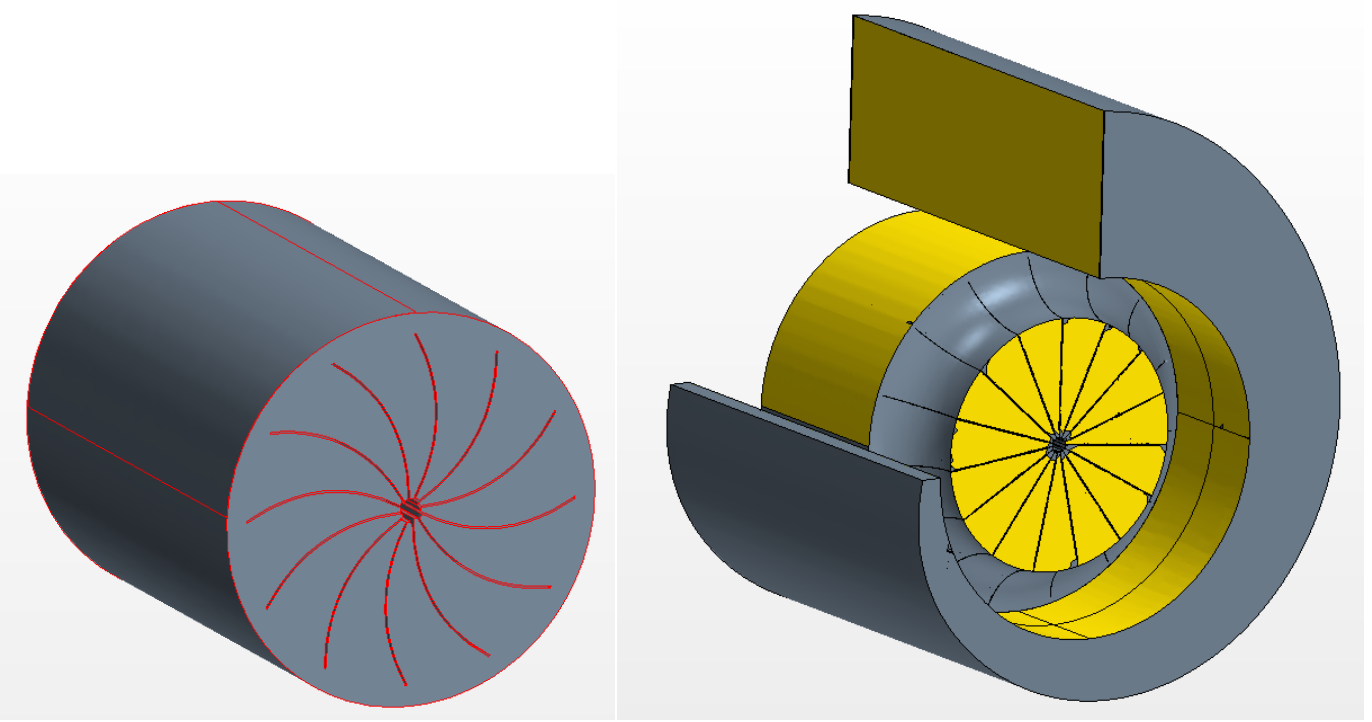

Figure 8.36: Geometry of the fan: initial (left) and updated (right)

The geometry was divided into several regions that have separate meshing settings. Nine regions for the tunnel, two regions for the fan assembly and one for the rest of the model were created. The densest mesh was defined in the fan region due to its geometrical complexity. A fine mesh was needed in order for the surface wrapper to fill the volume entirely. The entire model contained almost 3,500,000 cells. Several cross sections through it showing the mesh are shown in Figure 8.37. 


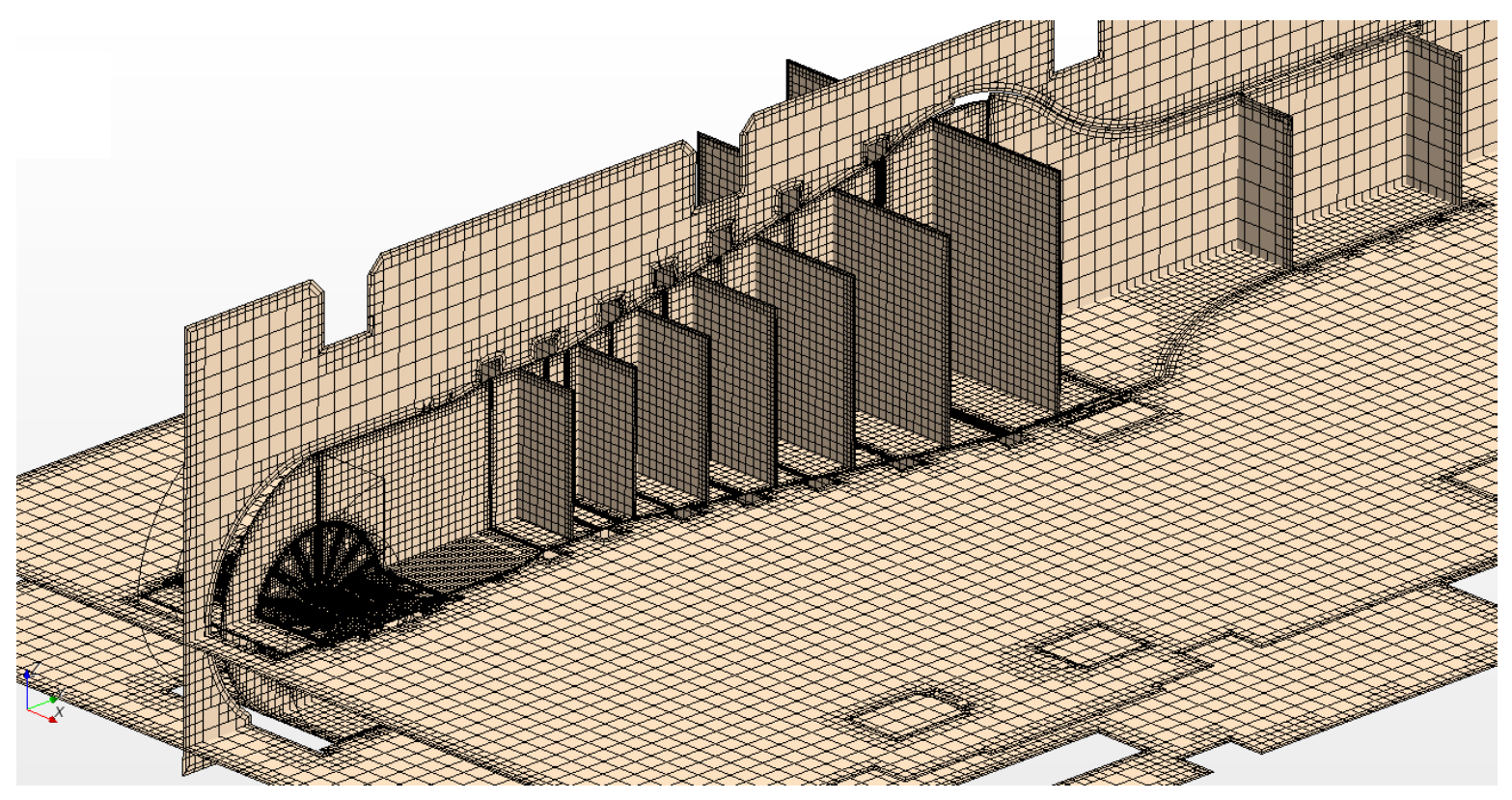

Figure 8.37: cross sections through the model

The interaction between the regions in the model was further defined by interfaces. The interfaces are highlighted in Figure 8.34 by yellow and orange colors. The yellow ones represent continuous in-place interfaces that do not cause any losses in the flow pressure while the orange ones represent porous baffles. The porous baffles were defined at the locations where screens are installed in the real tunnel. The porosity of the screens was determined to be 0.672 based on the specification sheets provided by TFHRC [1]. According to the STAR-CCM+ user guide [2] the pressure drop across a porous baffle can be modeled with the following equation:

$$
\Delta p=-\rho\left(\alpha\left|v_{n}\right|+\beta\right) v_{n}
$$

Where $\rho$ is the density of air and $v_{n}$ is the normal velocity of air acting on the screen and $\alpha$ and $B$ are parameters that depend on screen geometry and properties.

In [3], this relation is reduced to only the quadratic term with parameter $K$ :

$$
\Delta p=-\frac{1}{2} K \rho v_{n}^{2}
$$

Setting parameter $\beta$ to zero in Equation 8.6 we can now relate $\alpha$ to the $K$ parameter as:

$$
K=2 \alpha
$$

The simplest Borda-Carnot one-dimensional formula for screen pressure-drop coefficient defines $K$ as follows [4]:

$$
K=\frac{1-\chi}{\chi^{2}}
$$


Where $\chi$ is the open area ratio $(\chi=0.672)$.

As a first approximation parameters for the porous baffle were assumed constant for each screen as: $\alpha=0.3632$ and $\beta=0$. However, it should be noted that this formula may overestimate $K$ for high $\operatorname{Re}$ numbers [4]. A more advanced formula by Weighardt defines $K$ as [4]:

$$
K=6.5\left[\frac{1-\chi}{\chi^{2}}\right]\left[\frac{v_{n} d}{\chi v}\right]^{-0.33}
$$

Where $v_{n}$ is the air velocity normal to the screens, $d$ is the screen wire diameter and $v$ is the kinematic viscosity of the air.

Taking the diameter of the screen wire as 0.0075 inch, the kinematic viscosity of air as $15.68 \mathrm{e}-6 \mathrm{~m} / \mathrm{s}^{2}$ and its constant velocity on all screens to be $20 \mathrm{~m} / \mathrm{s}$, this formula yields definition:

$$
K=0.91 \frac{1-\chi}{\chi^{2}}
$$

This simple calculation shows that the constant $K$ may be substantially overestimated depending on the velocity of the air on the screen. Now that the approximate velocities of the air on the screens are known, a second iteration can be conducted with more accurate values for $K$ calculated from Equation 8.10 .

Another important feature of the model was the rotating fan. To introduce the rotation effect on the flow, a rotating reference system was defined with one axis going through the axis of the fan. A constant velocity of 500 rotations per minute was assigned to the system and the fan. This is the operating speed of the fan as per TFHRC specifications.

\subsubsection{Results}

\subsection{The model without the furniture}

The first set of simulations model the system without the furniture in the room to determine the characteristics of the flow field in an empty room as a base case. Figure 8.38 shows velocity profiles in the room and the tunnel at a vertical plane through the center of the tunnel. Note that the velocity in the extension of the tunnel and the testing section (just in the front of the tunnel) appears quite uniform. In a horizontal plane about mid-height through the tunnel the flow in the room is asymmetrical (see Figure 8.39). The left side (looking downstream) is blocked by the turbulence generator. In the original model the vanes in the generator were in a closed position. The mass flow through the fan inlet on the left side was $18.713 \mathrm{~kg} / \mathrm{s}(15.54 \mathrm{~m} \wedge 3 / \mathrm{s})$ and $17.058 \mathrm{~kg} / \mathrm{s}\left(14.17 \mathrm{~m}^{\wedge} 3 / \mathrm{s}\right)$ on the right side. The right side inlet is obstructed with a pulley plate for turning the fan. 


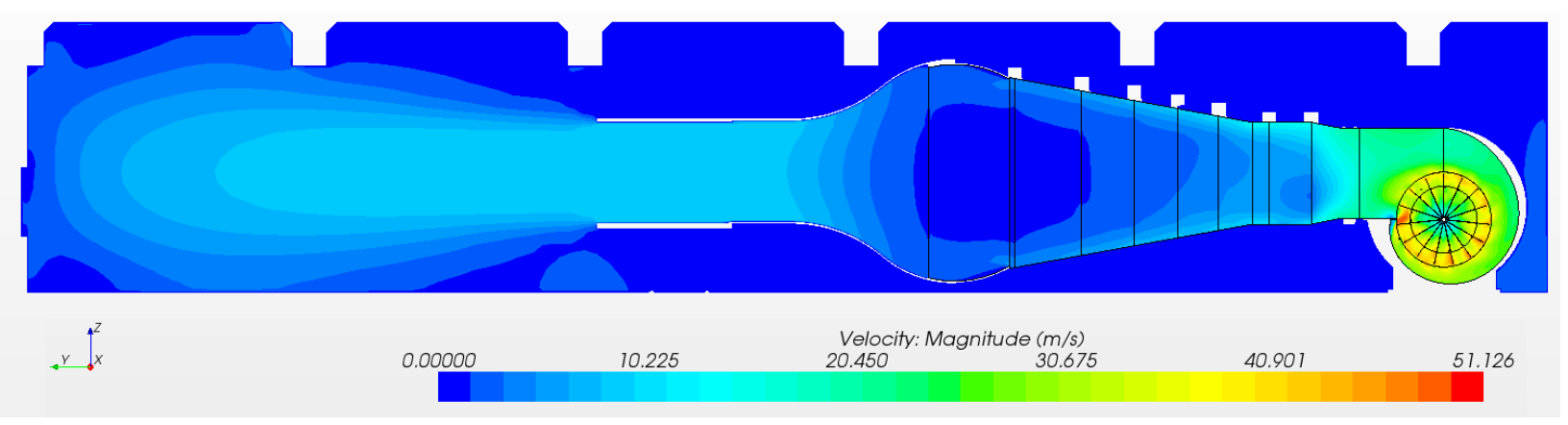

Figure 8.38: Velocity profile in model without the furniture - vertical plane

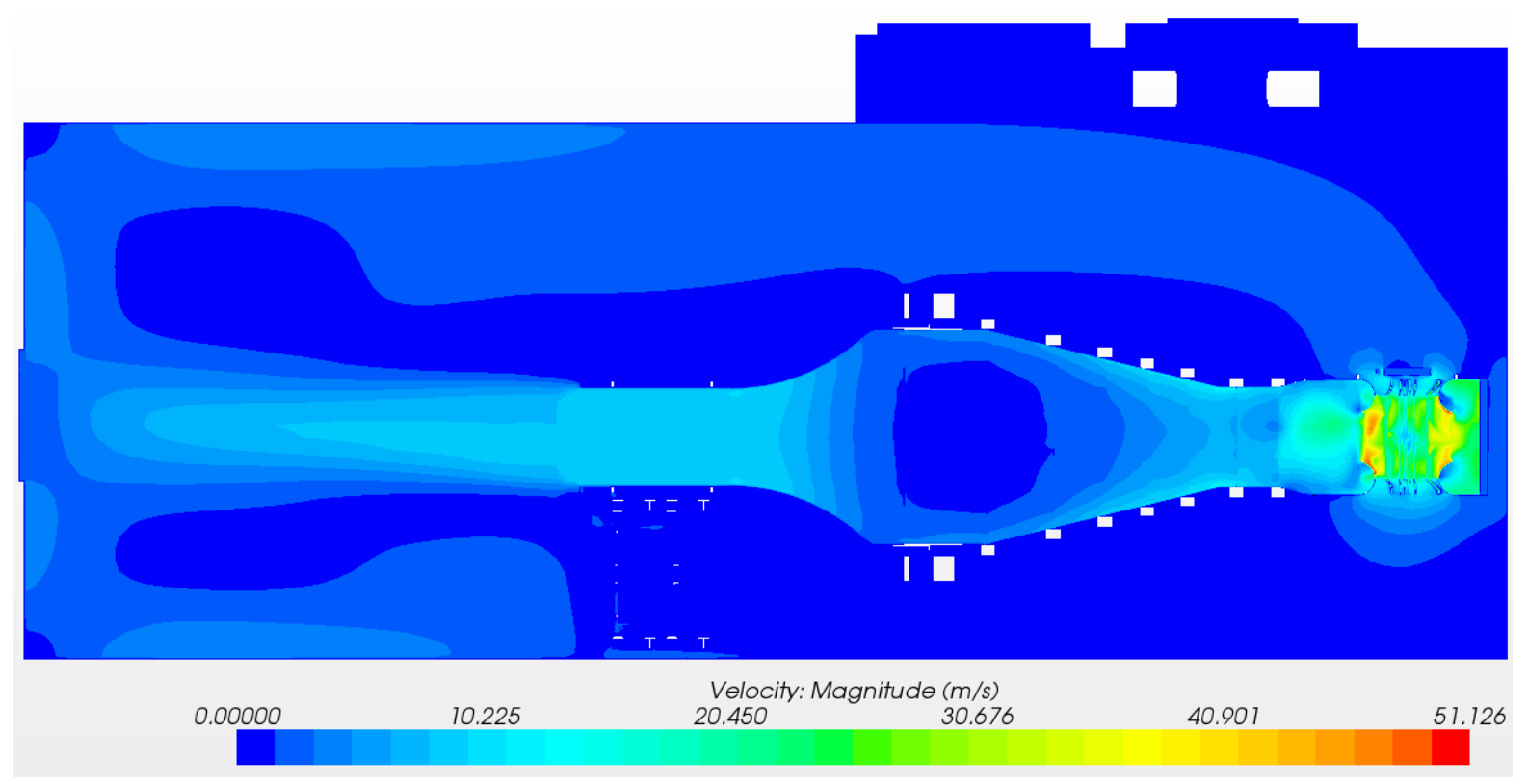

Figure 8.39: Velocity profile in model without the furniture - horizontal plane

Figure 8.40 presents the pressure profile in the wind tunnel. Significant drops of pressure are noted as expected across the screens.

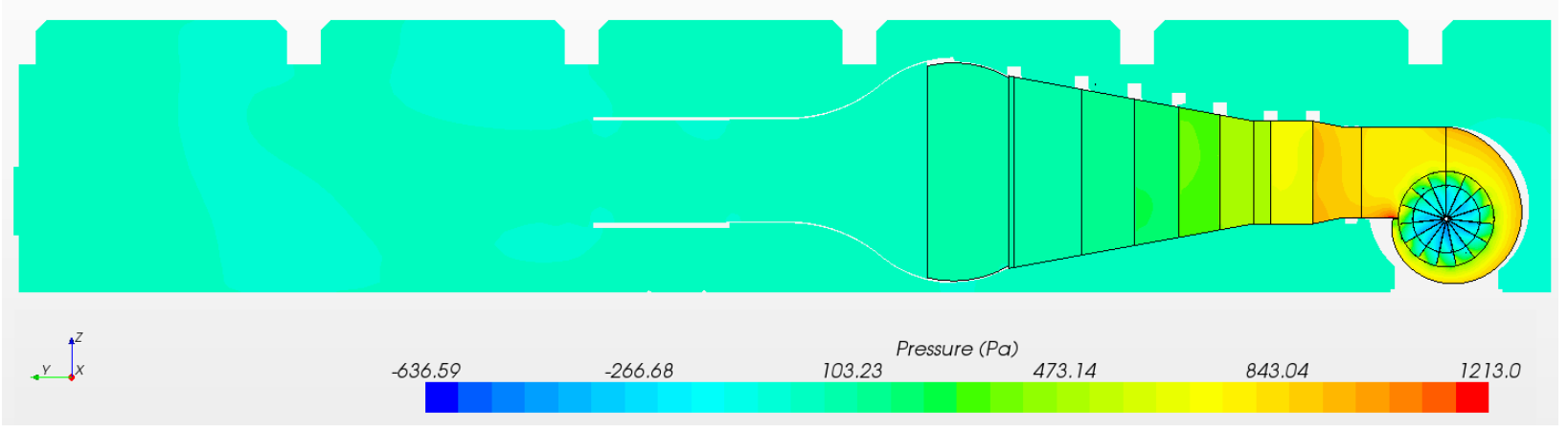

Figure 8.40: Pressure profile in the wind tunnel

To check how the turbulence generator is influencing the flow, the vanes in it were rotated to their neutral position. The generator itself was not moved from its initial position on the left side of the 
extension part of the tunnel. In this model the mass flow has changed only slightly: from $17.058 \mathrm{~kg} / \mathrm{s}$ $\left(14.17 \mathrm{~m}^{\wedge} 3 / \mathrm{s}\right)$ to $16.63 \mathrm{~kg} / \mathrm{s}\left(14.04 \mathrm{~m}^{\wedge} 3 / \mathrm{s}\right)$ on the side blocked with the fan pulley plate and from 18.713 $\mathrm{kg} / \mathrm{s}\left(15.54 \mathrm{~m}^{\wedge} 3 / \mathrm{s}\right)$ to $19.25 \mathrm{~kg} / \mathrm{s}\left(16.25 \mathrm{~m}^{\wedge} 3 / \mathrm{s}\right)$ on the open (left) side.

\subsection{The model without the furniture with removed screens in the tunnel}

In the initial simulations the velocity of the air was higher in the tunnel in the near downstream of the fan near the boundaries of the tunnel. This appears to be counterintuitive at first and additional simulation was performed where the screens - porous baffles - were replaced with the in-place interfaces to eliminate them as the cause of the flow distribution. With no porous baffles, there is no pressure drop across the interfaces at the tunnel sections in the model. This simplified model was supposed to provide more insight into the flow and help identify if any potential errors were produced by the definitions of the porous baffles. Figure 8.41 and Figure 8.42 show the velocity profile obtained from the simulation with this simplified model. Note that for this case the velocity is still highest on the top of the tunnel. This behavior is attributable to the rotating elements of the fan releasing the air at tangent to the fan's circumference. On the bottom wall of the tunnel the velocity is also high since the air coming off the fan blades is meeting an obstacle. The flow inside of the tunnel and in the testing section is highly non-uniform. This shows the need for the screens inside of the tunnel to produce a near uniform flow in the downstream. 


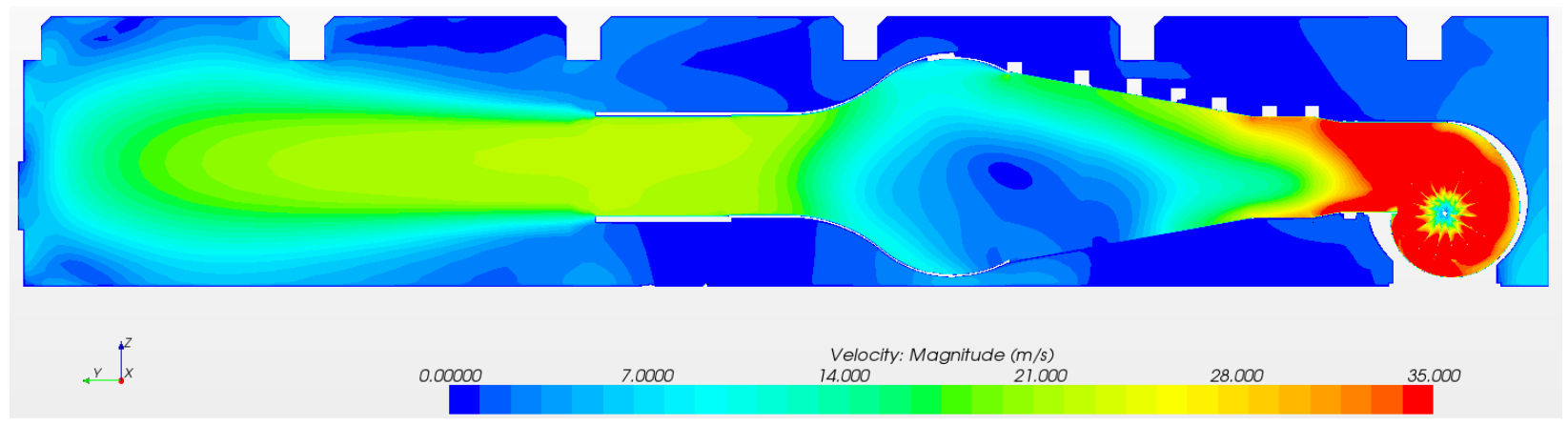

Figure 8.41: Velocity profile in model without the furniture and no screens - vertical plane

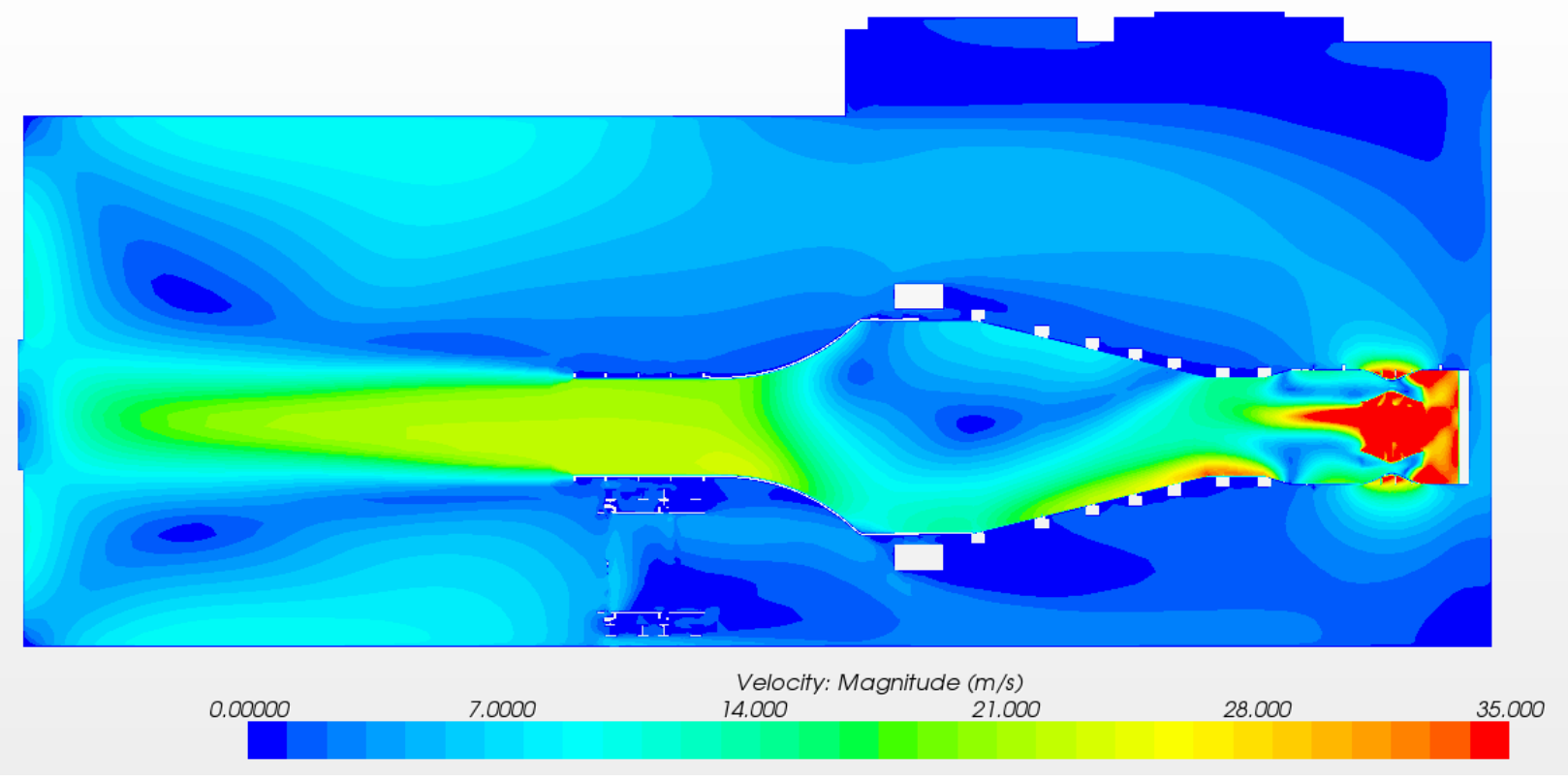

Figure 8.42: Velocity profile in model without the furniture and no screens - horizontal plane

The lack of the screens contributed significantly to the increase of the air mass flow through the fan inlets. The mass flow rate increased to $39.06 \mathrm{~kg} / \mathrm{s}\left(32.98 \mathrm{~m}^{\wedge} 3 / \mathrm{s}\right)$ and to $47.32 \mathrm{~kg} / \mathrm{s}(39.96 \mathrm{~m} \wedge 3 / \mathrm{s})$ on the right and left sides respectively.

\subsection{The model without the furniture with extended boundaries}

The next test conducted on the model was with a symmetric room where the walls were moved away from the tunnel, 10 meters each. Also the roof was raised 5 meters. Additionally the turbulence generator was removed from the model. This test was conducted to see how "ideal" conditions would change the flow pattern. Figure 8.43 shows the horizontal cross section through the model. The velocity profile is more symmetric than in the previous cases. The dark blue spans a velocity range between 0 and a little less than $2 \mathrm{~m} / \mathrm{s}$, causing the return flow not to show up in the plot because it is less than 2 $\mathrm{m} / \mathrm{s}$. 


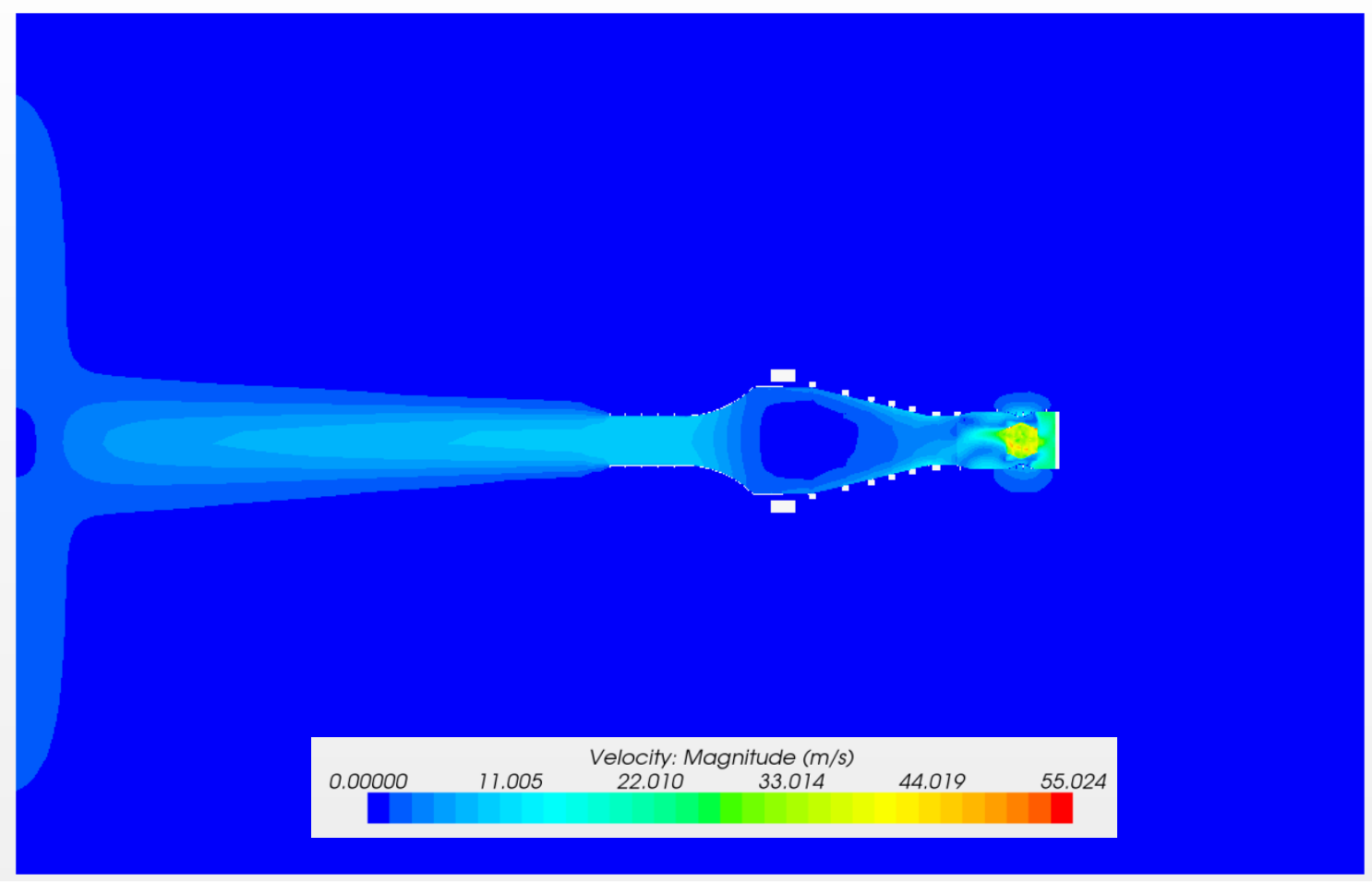

Figure 8.43: Velocity profile in model extended boundaries - horizontal plane

Surprisingly the air mass flow through the fan inlets didn't change much in comparison to the initial models. It has changed from $17.058 \mathrm{~kg} / \mathrm{s}\left(14.17 \mathrm{~m}^{\wedge} 3 / \mathrm{s}\right)$ to $16.70 \mathrm{~kg} / \mathrm{s}\left(14.10 \mathrm{~m}^{\wedge} 3 / \mathrm{s}\right)$ on the side blocked with the fan pulley plate (right side) and from $18.713 \mathrm{~kg} / \mathrm{s}\left(15.54 \mathrm{~m}^{\wedge} 3 / \mathrm{s}\right)$ to $18.87 \mathrm{~kg} / \mathrm{s}\left(15.94 \mathrm{~m}^{\wedge} 3 / \mathrm{s}\right)$ on the left side.

\subsection{Comparison of the flow characteristics}

In order to quantify the changes in the flow through the tunnel area, a standard deviation and coefficient of variation of the velocity at nine cross sections in the tunnel were calculated. The location of nine cross sections of interest is shown in Figure 8.44. Seven of them were located between the screens and additional two in the tunnel extension. 


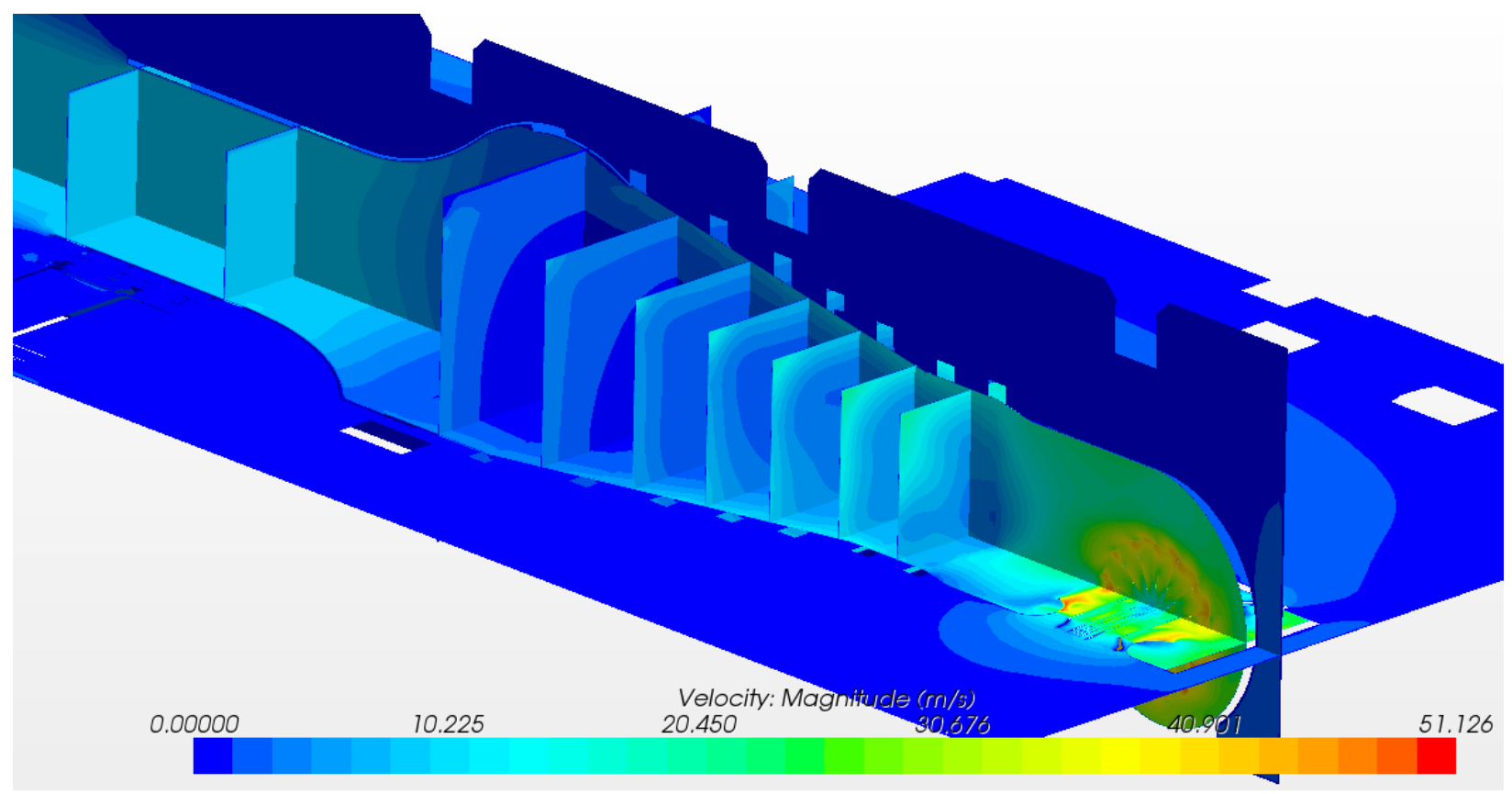

Figure 8.44: Sections of interest in the tunnel

Area averaged standard deviation of the velocity for three models:

- initial, without the furniture, with the turbulence generator vanes closed

- initial, without the furniture, with the turbulence generator vanes in neutral position

- without the furniture with extended boundaries, without the turbulence generator

are plotted in Figure 8.45. It can be noted that a slight difference between the curves can only be noticed at the first two screens. The standard deviation does not take into account the fact that the mean velocity is dropping once we go through the screens and for that reason the coefficient of variation of the velocity was also calculated. In both cases the screens smear out nearly all the nonuniformities of the velocity profile in the tunnel, reducing the measure of variation by about an order of magnitude. 


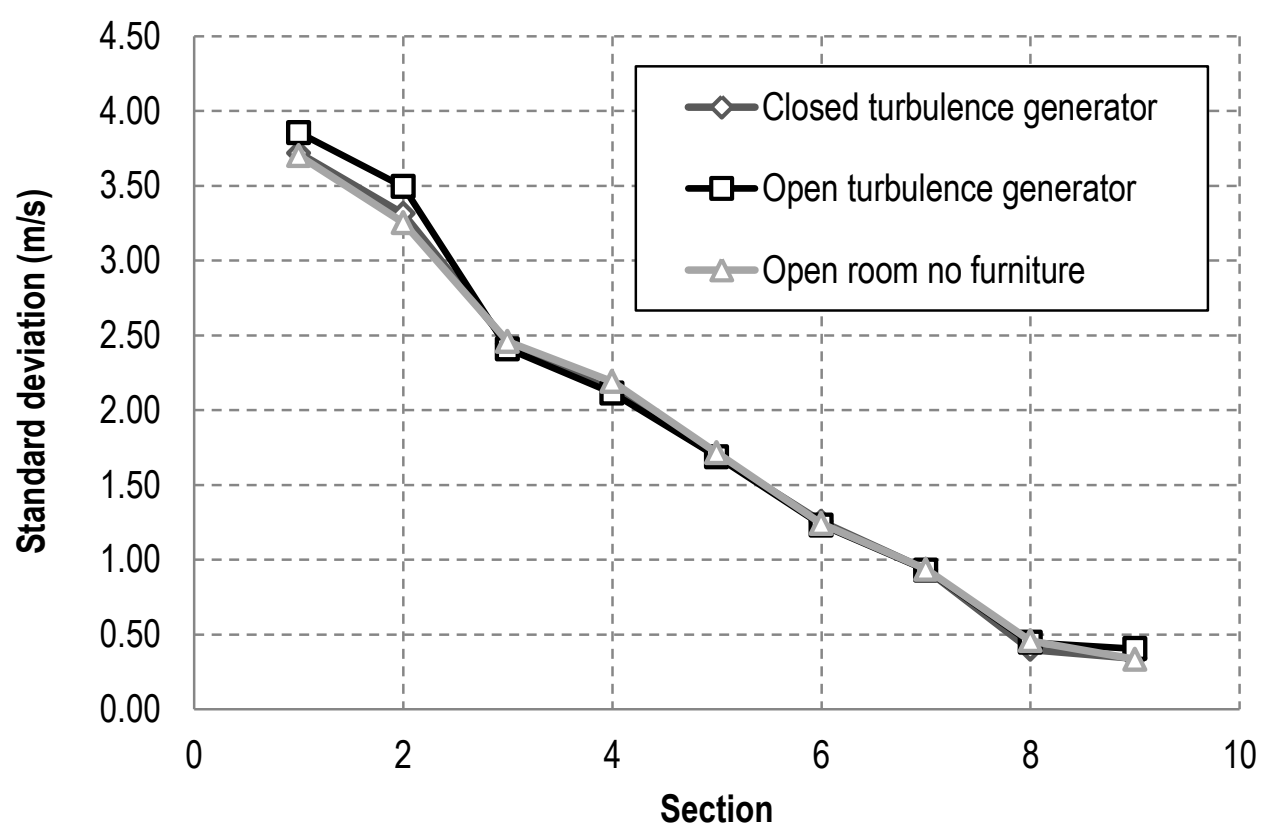

Figure 8.45: Area averaged standard deviation of the air velocity in the tunnel

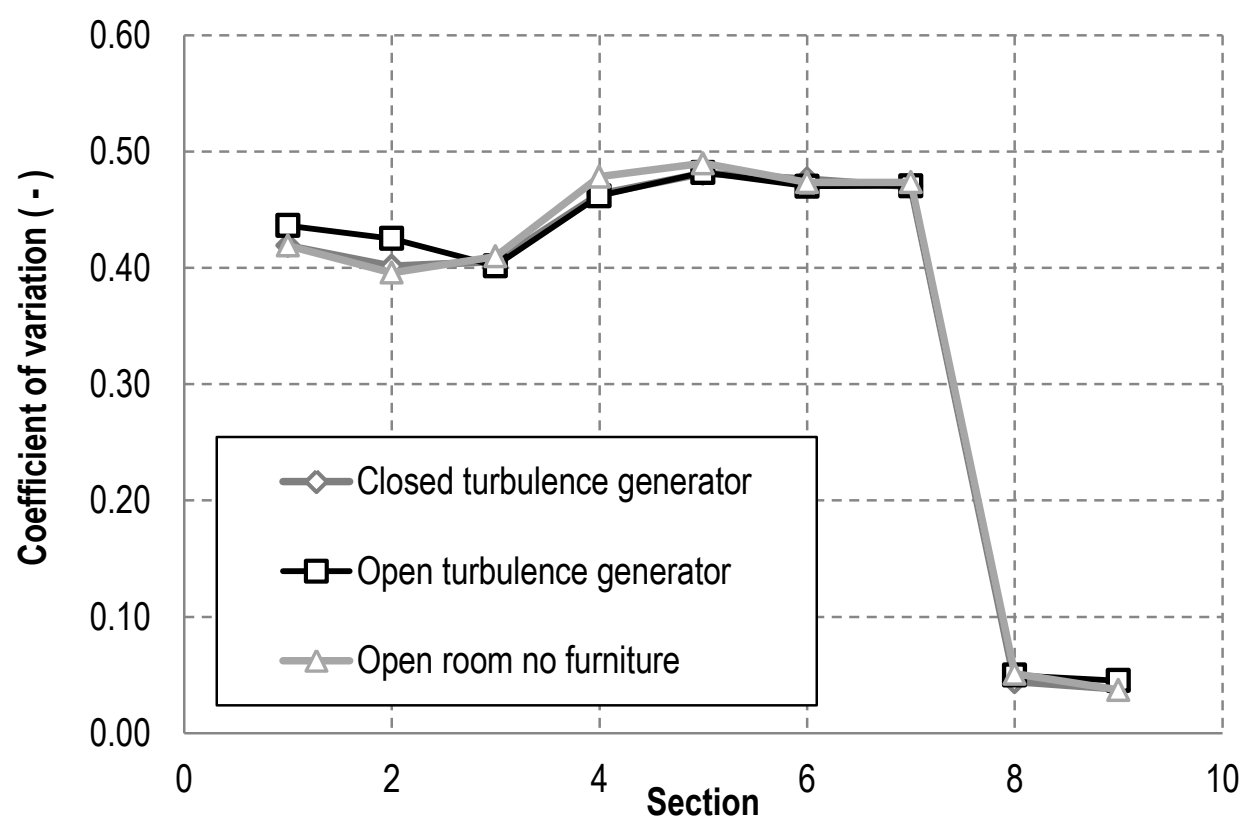

Figure 8.46: Area averaged coefficient of variation of the air velocity in the tunnel

Table 8.3 lists the mass flow rate calculated for the same three models. Again it can be noticed that despite the fact of considerable change in the model - translation of all the walls and removal of the turbulence generator, the amount of the air going through the inlets is not changing significantly. 
Table 8.3: Mass flow rate through the fan inlets

\begin{tabular}{|l|l|l|}
\hline Model & Left side & Right side \\
\hline Closed turbulence generator & $18.713 \mathrm{~kg} / \mathrm{s}\left(15.54 \mathrm{~m}^{\wedge} 3 / \mathrm{s}\right)$ & $17.058 \mathrm{~kg} / \mathrm{s}\left(14.17 \mathrm{~m}^{\wedge} 3 / \mathrm{s}\right)$ \\
\hline Open turbulence generator & $19.250 \mathrm{~kg} / \mathrm{s}\left(16.25 \mathrm{~m}^{\wedge} 3 / \mathrm{s}\right)$ & $16.630 \mathrm{~kg} / \mathrm{s}\left(14.04 \mathrm{~m}^{\wedge} 3 / \mathrm{s}\right)$ \\
\hline Open room & $18.870 \mathrm{~kg} / \mathrm{s}\left(15.94 \mathrm{~m}^{\wedge} 3 / \mathrm{s}\right)$ & $16.700 \mathrm{~kg} / \mathrm{s}\left(14.10 \mathrm{~m}^{\wedge} 3 / \mathrm{s}\right)$ \\
\hline
\end{tabular}

To summarize, the flow through the tunnel appears to be insensitive to the changes in the room geometry that were tested in the current quarter. Yet additional runs for the cases with fully furnished room need to be performed to check the magnitude of the effect of current room furniture on the air flow uniformity. Note that the detailed model of the fan would also influence the results. However, the big picture is expected to remain largely unchanged with minor changes to the fan model geometry.

\subsubsection{References}

[1] McMaster-Carr, http://www.mcmaster.com/\#wire-mesh/=emqkhc

[2] CD-adapco, User Guide STAR-CCM+ Version 6.02.008, 2011

[3] R.D. Mehta, Turbulent Boundary Layer Perturbed by a Screen, AIAA Journal, Vol. 2, No. 9, pp 1335-1342, September 1985

[4] R.D. Mehta, P. Bradshaw, Design rules for small low speed wind tunnels, The Aeronautical Journal of the Royal Aeronautical Society, November 1979 


\subsubsection{Modeling of Truck Generated Salt Spray under Bridge with Sliding Mesh}

\subsubsection{Model development}

\subsection{Geometry}

In the recent quarter, a CFD approach to the modeling of truck generated salt spray was initiated. The technique of using a sliding mesh was employed to introduce movement of the vehicle. Domains with the truck and the bridge were separately built and meshed. A denser mesh with base size for an edge of $0.1 \mathrm{~m}$ was defined for the area surrounding the truck and the bridge beams. The rest of the domain was meshed with the base size of an element equal to $0.5 \mathrm{~m}$. The geometry of the Raleigh - Tamarack Overpass (Bridge No. 4172) was used in the CFD analysis. The model of a truck tractor was based on the Mack CH 613 tractor. The sleeper and the dry freight van type trailer were modeled previously for the LS-DYNA analysis. The biggest allowed - $16.16 \mathrm{~m}$ long and $2.89 \mathrm{~m}$ wide - trailer was modeled.

From the previous analysis in LS-DYNA it was learned that a large volume behind the truck needs to be modeled in order to capture important behavior of the air behind the truck. For that reason, an air domain of $170 \mathrm{~m} \times 42.8 \mathrm{~m} \times 7.6 \mathrm{~m}$ was modeled in the CFD study. The meshing settings and extent of the model resulted in an overall computational cell count of $3,600,000$. Figure 8.47 shows the geometry of the model at an intermediate stage of the analysis.

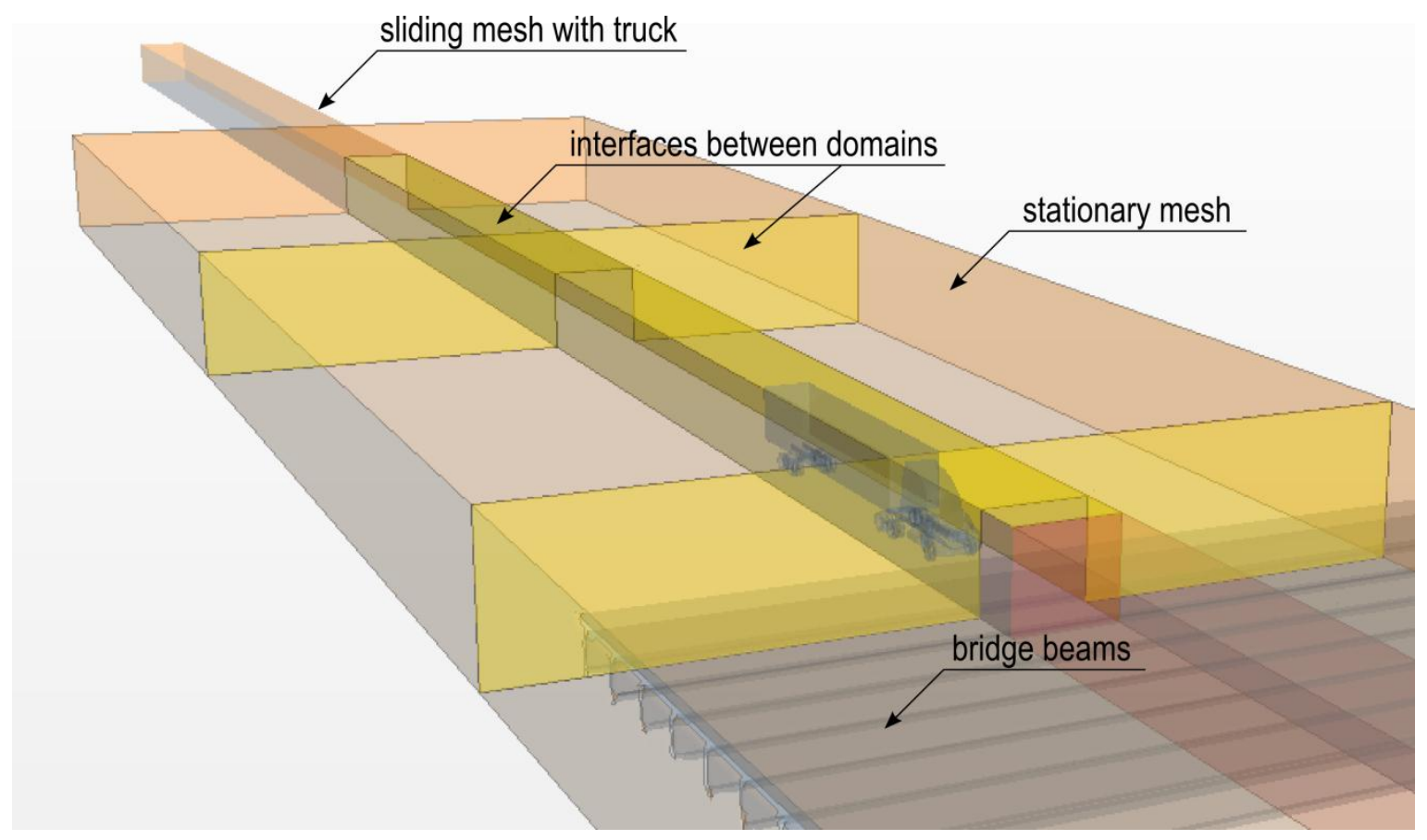

Figure 8.47: Geometry of the STAR-CCM+ model for analysis of truck generated salt spray

Initially the box with the truck was placed about $61.5 \mathrm{~m}$ in the front of the bridge. That distance was assumed to be enough for the flow around the vehicle to fully develop. Openings in the air domain with exact size of the truck box were created. The sliding mesh with the vehicle perfectly fills that volume once the vehicle is set in motion. In implicit unsteady analysis a time step of $0.02 \mathrm{sec}$ was used. The 
number of inner iterations of the solver was set to 20 to converge each time step. Motion of the box with the vehicle was prescribed in the forward $-X$ direction with a velocity of $26.8 \mathrm{~m} / \mathrm{s}$ (60 mph). In each time step interfaces between the adjacent domains are recreated and flow between them is allowed.

\subsection{Spray generation}

Lagrangian particles were used in the model to represent the liquid particles released from the truck tires. STAR-CCM+ has built in several types of particle injectors that can be attached to the boundaries of the geometry:

- Part injector,

- Point injector,

- Cone injector (particles with mass only),

- Surface injector, and

- Pressure swirl injector.

While the cone injectors are most sophisticated [1] their use in our case would require definition of their locations for each time step of the calculation. These injectors cannot be easily attached to the moving mesh. The surface injectors do not require specification of their location each time the vehicle moves. Contrary to the cone and point injectors the surface injector is always attached to the underlying geometry. Velocity magnitude and direction are specified for particles with mass released by the surface injectors. Massless particles are injected normal to surface and their velocity is not specified.

Probably the most difficult part of this analysis is to determine initial conditions for the generated sprays. Particle size distribution, particle velocity magnitude, its direction, and particle breakup properties are the key factors in the analysis. The literature on this topic is very limited since any experiments determining generation of a spray by rolling tires are difficult to conduct. In [2] tests with an original Tire Spray Simulator are presented as a source of information on the creation of a spray from tires. In the experiments an apparatus like the one shown in Figure 8.48 was used. Two tires were pressed against each other - one of them had a greatly simplified tread in the form of a single circumferential groove, and the other one had no tread to simulate a smooth road surface. Water was injected between the rolling tires and spray generated behind them was analyzed using digital particle imaging velocimetry technique (DPIV). Particle size distribution, the mean diameter and the breakup length were shown to depend on the Weber number. Measurements for the breakup length of the liquid sheet showed a dependence on Weber number proportional to $\mathrm{We}^{-1 / 6}$. The range of droplet sizes and the mean diameter were found to decrease with Weber number by approximately $\mathrm{We}^{-1 / 2}[2]$. 


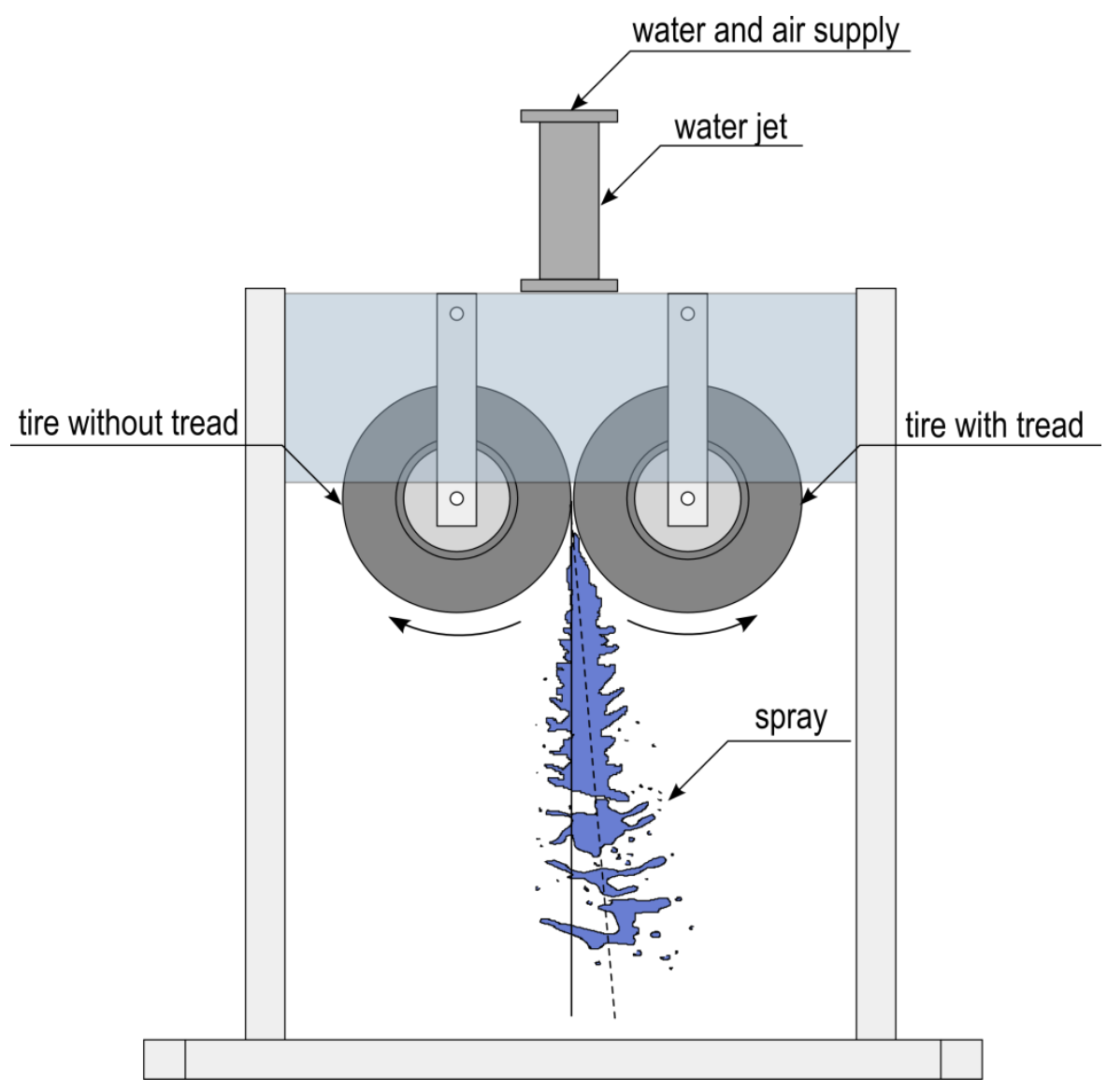

Figure 8.48: Schematic drawing of the Tire Spray Generator (based on [2])

Weber number in the test depends on properties of the water, tire speed and the tire groove width. For different Weber numbers different particle distributions were obtained at several locations behind the tires. In all tested cases the distribution of particles can be described by a log-normal distribution. One of such distributions is shown in Figure 8.49. The following approximate mean particle sizes were observed in the spray coming off the tire at a distance of 1.5 tire diameters from the end of the contact patch:

- For the lowest $W e=2,700$ distributions had a mean particle size of approximately $750 \mu$.

- For $W e=10,900$ distributions had a mean particle size of approximately $400 \mu$.

- For the highest $W e=24,400$ distributions had a mean particle size of approximately $300 \mu$.

$W e=24,400$ was associated with the tire surface velocity of $18 \mathrm{~m} / \mathrm{s}$. In our case the velocity of the tire surface is $26.8 \mathrm{~m} / \mathrm{s}$. Also the tire geometry is substantially different since commercial tires have multiple, circumferential and non-circumferential groves in a variety of tread patterns and larger diameter than the tested tire. Thus, the results listed here cannot be directly used as an input for the analysis of spray distribution coming from large trucks. Nevertheless, the mean values of the particle diameters were used as a basis for the further study. 


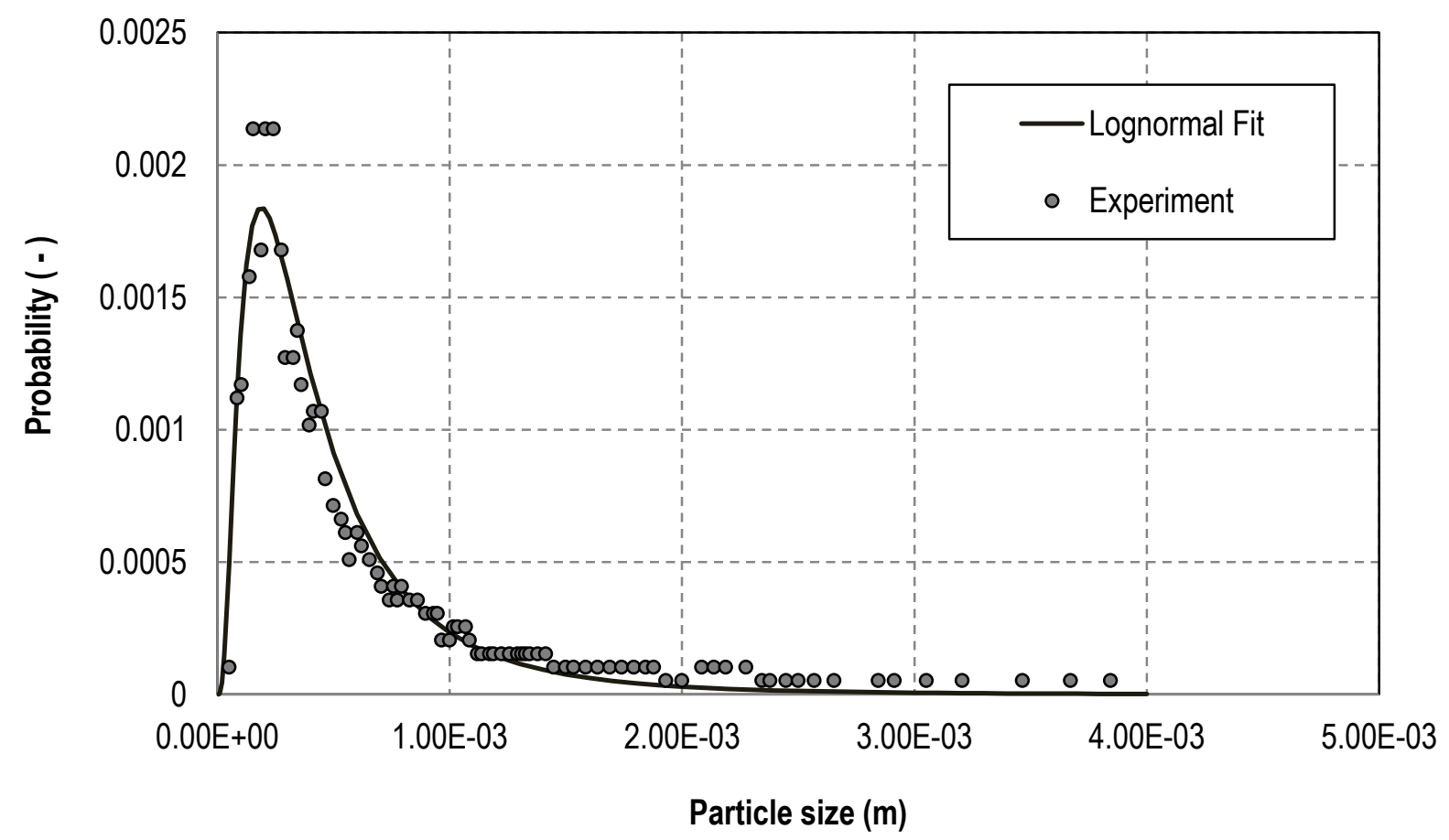

Figure 8.49: Particle size distribution in Tire Spray Generator test [2]

One set of simulations were conducted on a stationary vehicle ("sliding mesh with truck" from Figure 8.47) without the air domain and the bridge model. Of interest in this run was just the breakup of the particles due to their impact with the wheel well. A goal of this simulation was to find what size particles can be expected to leave the wheel well zone after the breakup. This size range is then used as a droplet injection boundary condition in the simulations of a moving vehicle, where it is too computationally expensive to use the breakup model.

The Bai-Gosman wall impingement model was activated in these simulations. Depending on the Weber and Laplace numbers for the droplets, the Bai-Gosman model is simulating a wide range of behaviors of wall impacting particles like: sticking, rebound, or break-up upon rebound (see Figure 8.50) [3].

The Weber number characterizes the ratio of inertial to surface tension forces and is defined as:

$$
W e=\frac{\rho V^{2} D}{\sigma}
$$

Where $\rho$ is the carrier fluid density, $V$ the slip velocity between the droplet and the carrier fluid, $D$ is the drop diameter and $\sigma$ is the droplet surface tension.

The Laplace number is a dimensionless number used in the characterization of free surface fluid dynamics. It relates the inertial and surface tension forces of the droplet to the viscous forces. It is also described as a ratio of surface tension to the momentum-transport inside a fluid.

It is defined as: 


$$
L a=\frac{\rho \sigma D}{\mu^{2}}
$$

Where $\mu$ is viscosity of the droplet liquid.

The boundaries between different regimes are defined in the updated Bai-Gosman model [3] and listed in Table 8.4. Implementation of this model in STAR-CCM+ includes temperature dependency of these boundary values. The user also specifies number of child particles upon break-up of particle and angle range for possible rebound. The default values of two child particles and angle range of 5 to 55 deg was used for the current analysis.

Table 8.4: Regime transition criteria [3]

\begin{tabular}{|c|c|c|}
\hline Wall status & Regime transition state & Critical Weber number \\
\hline Dry & Adhesion to Splash & $W e=2630 \mathrm{La}^{-0.183}$ \\
\hline Wet & Stick to Rebound & $W e=2$ \\
\hline Wet & Rebound to Spread & $W e=20$ \\
\hline Wet & Spread to Splash & $W e=1320 \mathrm{La}^{-0.183}$ \\
\hline
\end{tabular}

stick

rebound
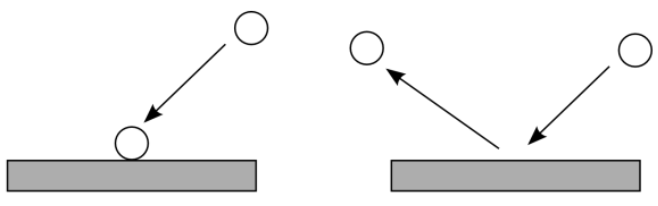

spread

break-up and rebound
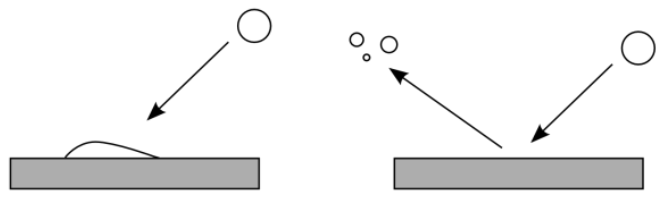

break-up and spread

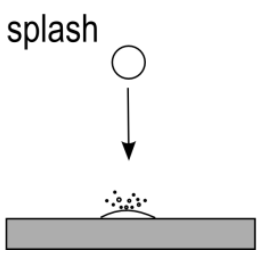

Figure 8.50: Possible particle behavior in Bai-Gosman model of wall impingement

In the simulation, droplets with a constant diameter were injected to the system from the tire tread surface. Three different sizes of particles were investigated, chosen based on the study presented in [2]: $0.3 \mathrm{~mm}, 0.5 \mathrm{~mm}, 0.7 \mathrm{~mm}$. The particles were injected with velocity of $26.8 \mathrm{~m} / \mathrm{s}$ (60 mph) at an angle of 15 degrees to the ground in the plane perpendicular to the wheel axles. The vehicle was considered 
stationary but the rotation of the tire, movement of the bottom boundary (ground) and movement of the air was defined to represent motion of the vehicle with a velocity of $26.8 \mathrm{~m} / \mathrm{s}$ (60 mph).

Currently the simulations for the $0.3 \mathrm{~mm}$ and $0.7 \mathrm{~mm}$ initial particle size are being run and these results will be shown in the next quarter. Only results for the size of $0.5 \mathrm{~mm}$ particles are reported. Figure 8.51 shows an intermediate state for this simulation. Droplets with injected size of $0.5 \mathrm{~mm}$ (red color) don't undergo breakup because they do not hit the wall of the wheel well. Droplets leaving the tire within the wheel well collide with the walls and breakup into a much smaller size before they are transported out via drag forces with the air flow. The droplets with smaller diameter (blue in figure) have a diameter around 100 microns.
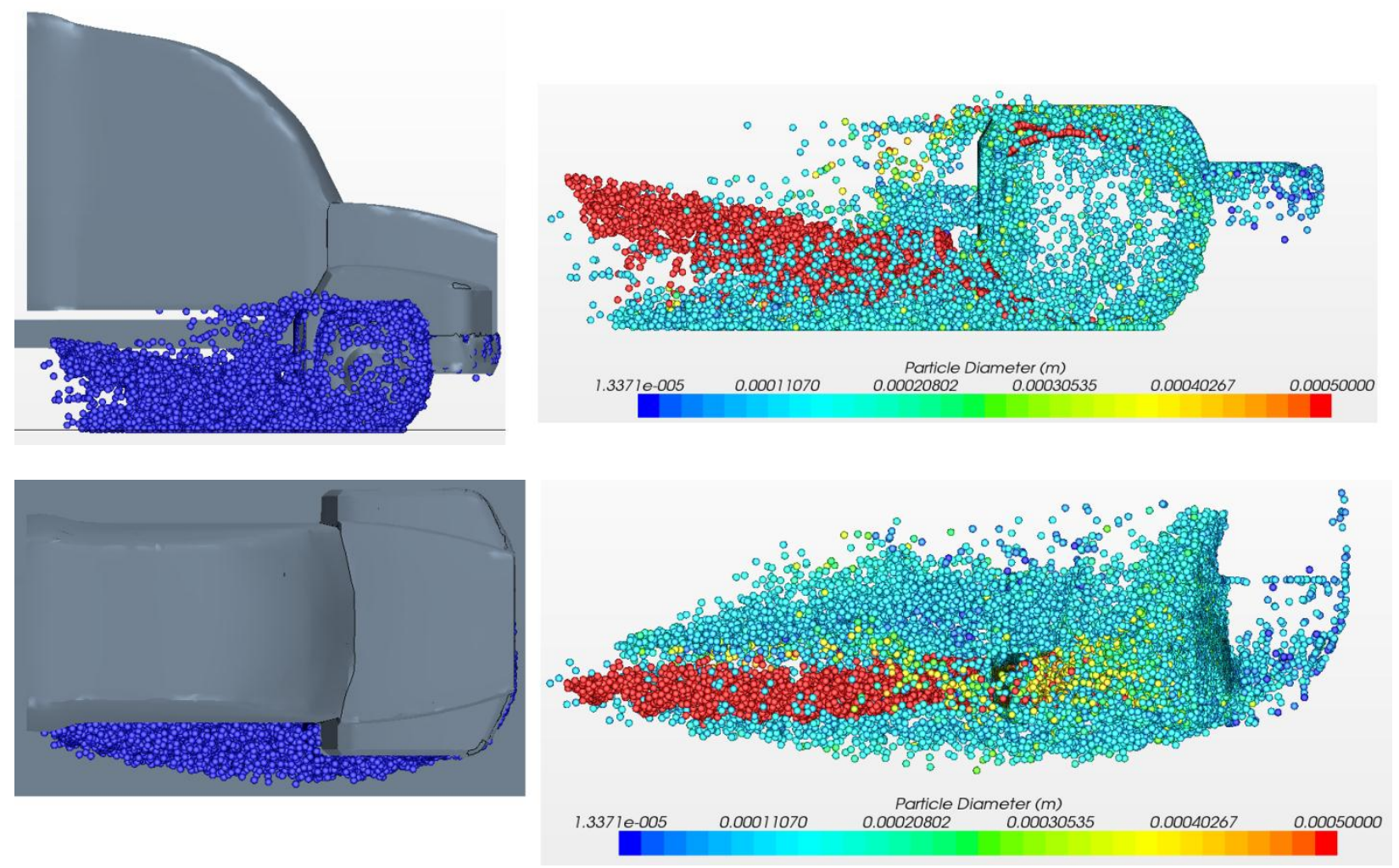

Figure 8.51: Particle breakup in the wheel well

Figure 8.52 shows the distribution of the particles after $0.5 \mathrm{sec}$ of simulation. A substantial number of droplets have the original size because they did not experience any collisions. However, the peak of the droplet diameter is now around the size of 90 microns. As can be seen in Figure 8.51 (top view) only droplets with size around 100 microns (blue color) are leaving the wheel well to the side. Bigger droplets will not leave this area unless there are no obstacles in their way. Usually mud flaps and sometimes other devices are installed around the truck wheels to prevent this from happening. Thus, for further analysis it is safe to assume that only droplets that have undergone breakup need to be considered. This conclusion is in agreement with the findings from the research conducted at Lawrence Livermore National Laboratory reported in [4]. 


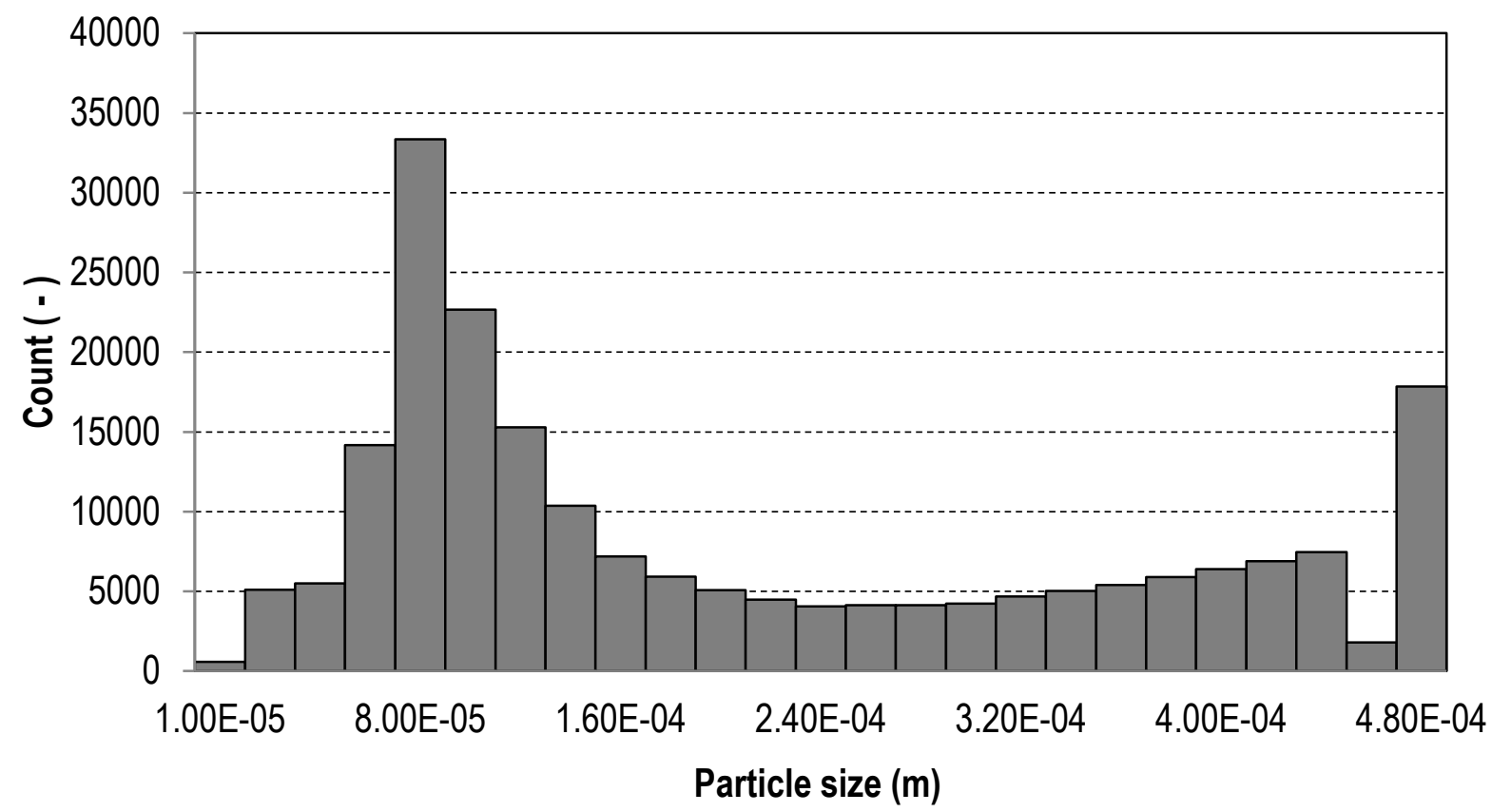

Figure 8.52: Particle distribution after break-up in the wheel well

\subsection{Simulations of the moving truck}

While the most important locations of the injectors are the wheel treads, a few cases have been run with the injectors attached to the cabin's edges. Figure 8.53 shows the location of the injector surfaces with surfaces at the rear side of the cabin. In Figure 8.54 droplets generated on these surfaces are displayed. 


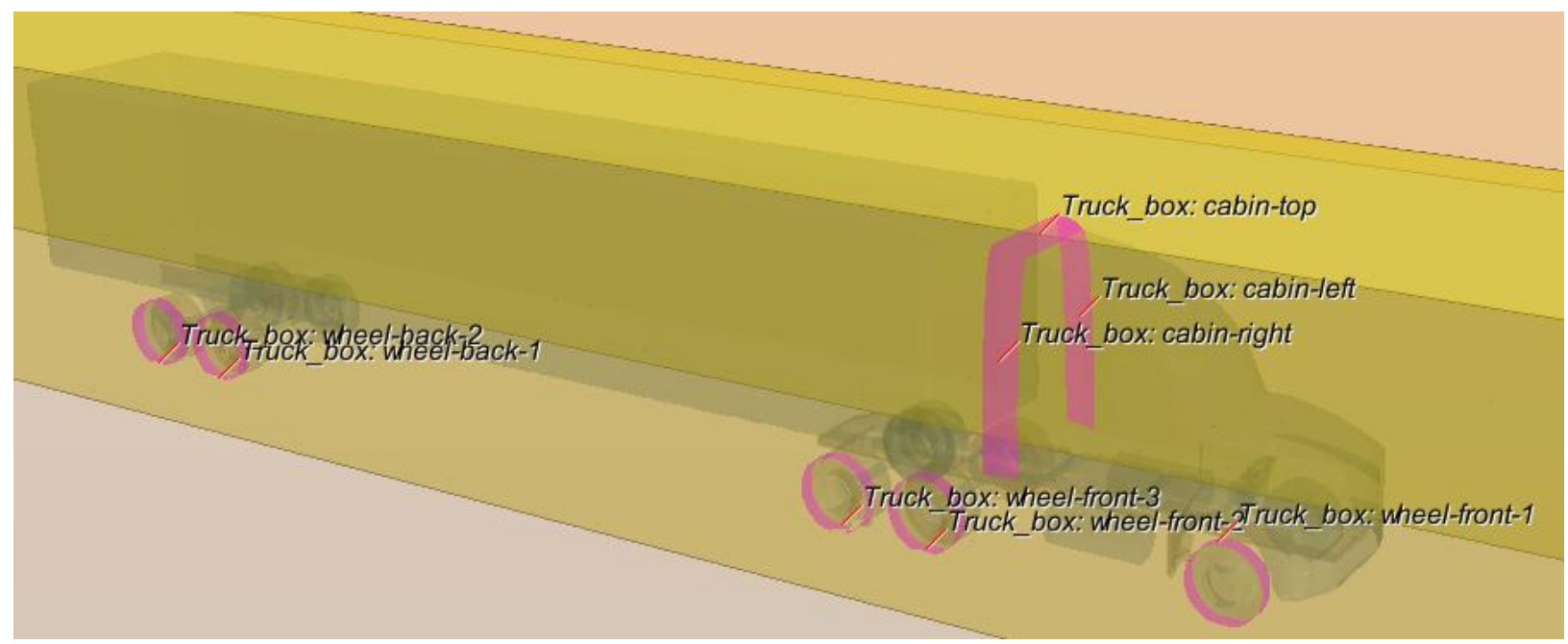

Figure 8.53: Location of surfaces used as surface injectors
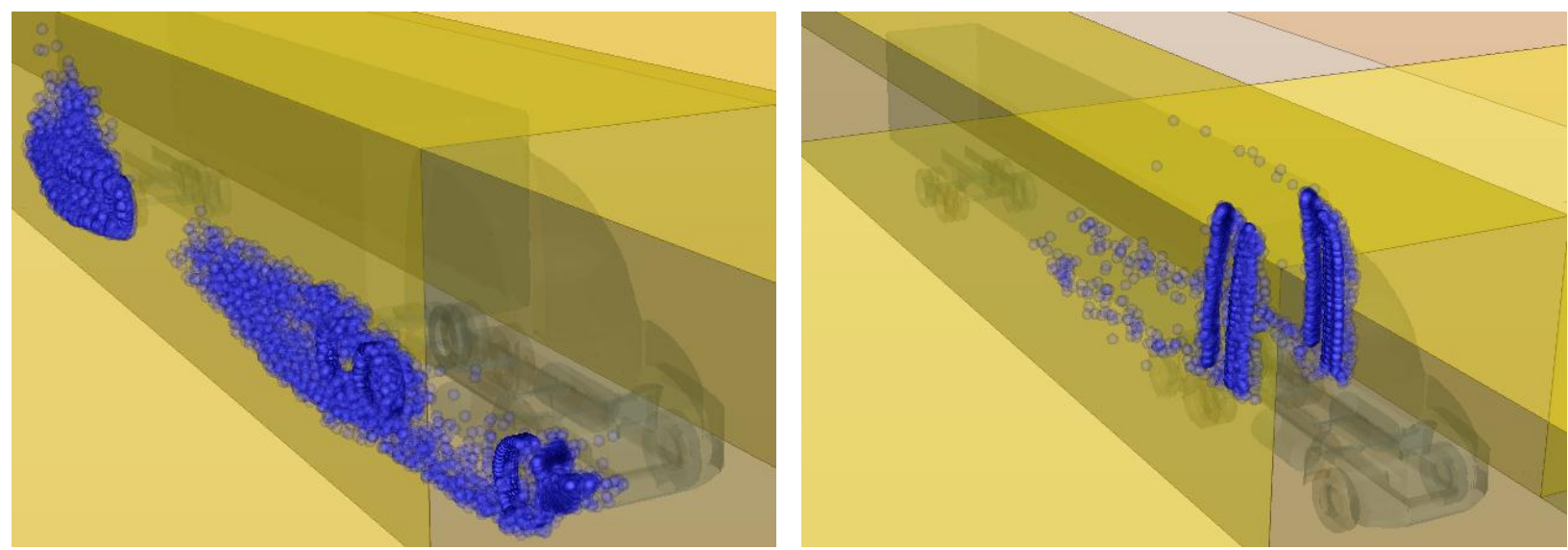

Figure 8.54: Particle generated on the injector surfaces

As the main goal of this study is to provide an assessment of possible travel paths of a salt spray generated by the tires to the bridge beams, several cases were analyzed. Table 8.5 lists completed cases and others, planned for analysis in the next quarter.

Table 8.5: List of analyzed cases

\begin{tabular}{|l|l|l|l|l|l|}
\hline case & particle size & \multicolumn{1}{|c|}{ injector angle } & \multicolumn{1}{|c|}{ injector location } & $\begin{array}{c}\text { boundary type under } \\
\text { the bridge }\end{array}$ & $\begin{array}{c}\text { number of } \\
\text { vehicles }\end{array}$ \\
\hline \multicolumn{7}{|c|}{ completed runs } \\
\hline 1 & massless & - & top surface of the cabin & open & one \\
\hline 2 & massless & - & side surfaces of the cabin & open & one \\
\hline 3 & 50 microns & $(-1.0,-1.0,0)$ & rear tire & open & one \\
\hline 4 & 100 microns & $(-1.0,-1.0,0)$ & rear tire & open & one \\
\hline 5 & 50 microns & $(-1.0,-0.1,1.0)$ & rear tire & open & one \\
\hline 6 & 100 microns & $(-1.0,-0.1,1.0)$ & rear tire & open & one \\
\hline
\end{tabular}




\begin{tabular}{|l|l|l|l|l|l|}
\hline 7 & 100 microns & $(-1.0,0.0,0.268)$ & all tires & open & one \\
\hline 8 & 100 microns & $(-1.0,0.0,0.268)$ & all tires & closed & one \\
\hline \multicolumn{7}{|c|}{ in progress or planned runs } \\
\hline 9 & 100 microns & $(-1.0,0.0,0.268)$ & all tires & open & two \\
\hline 10 & 100 microns & $(-1.0,0.0,0.268)$ & all tires & closed & two \\
\hline 11 & 100 microns & $(-1.0,0.0,0.268)$ & all tires & sloped & two \\
\hline
\end{tabular}

\subsection{Massless particles released on the edges of cabin}

In the first set of simulations massless particles were injected at the edges of the cabin. As shown in Figure 8.55, the droplets did not reach the level of bridge beams in any of the two analyzed cases of injectors on the top and sides of the cabin. Massless droplets strictly follow the streamlines of the flow and without additional flow disturbances i.e. another vehicle or wind, do not reach the bridge beams.

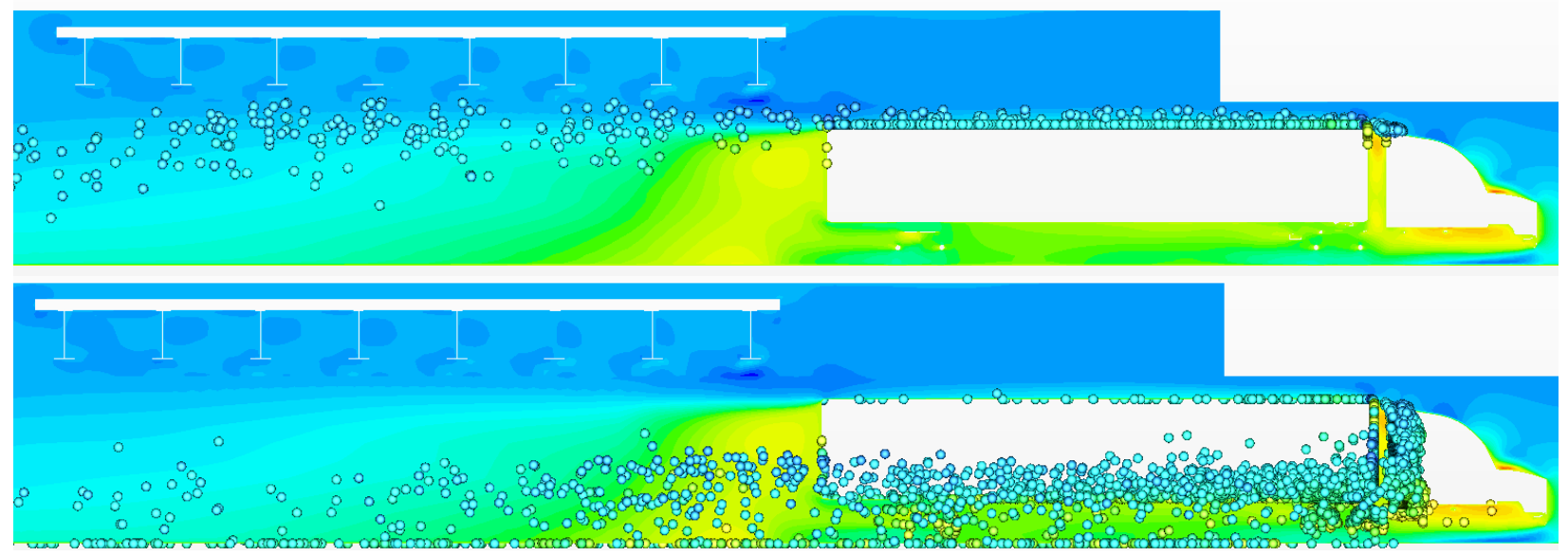

Figure 8.55: Velocity field around the truck and distribution of massless droplets injected at the cabin's edges

\subsection{Droplets with mass released at the rear tires to the side of the truck}

Droplet tracking is computationally expensive and requires very long run times (many days) when the number of droplets is large. For this reason, the simulations reported here have injection droplets from the rear wheel and all of the wheels on one side of the truck. This approach to studying droplet transport is reasonable because the number of droplet-droplet interactions is negligible and droplet number densities in the sprays from truck tires are not high enough to substantially alter the air flow. The initial models do not have mud flaps installed since the break-up of the droplets was not included here. First an angle of 45 degrees in the horizontal plane was assumed for injected droplets - vector ($1.0,-1.0,0)$. Droplets with a diameter of 50 microns were injected at velocity of $26.8 \mathrm{~m} / \mathrm{s}(60 \mathrm{mph}$ ). Figure 8.56 shows the tire spray droplet distribution of this simulation when the truck is already passed the bridge. Droplets thrown to the side are raised by the moving air to about half of height of the truck. The presence of the bridge does not change behavior of the droplets to a significant extent in this case. 

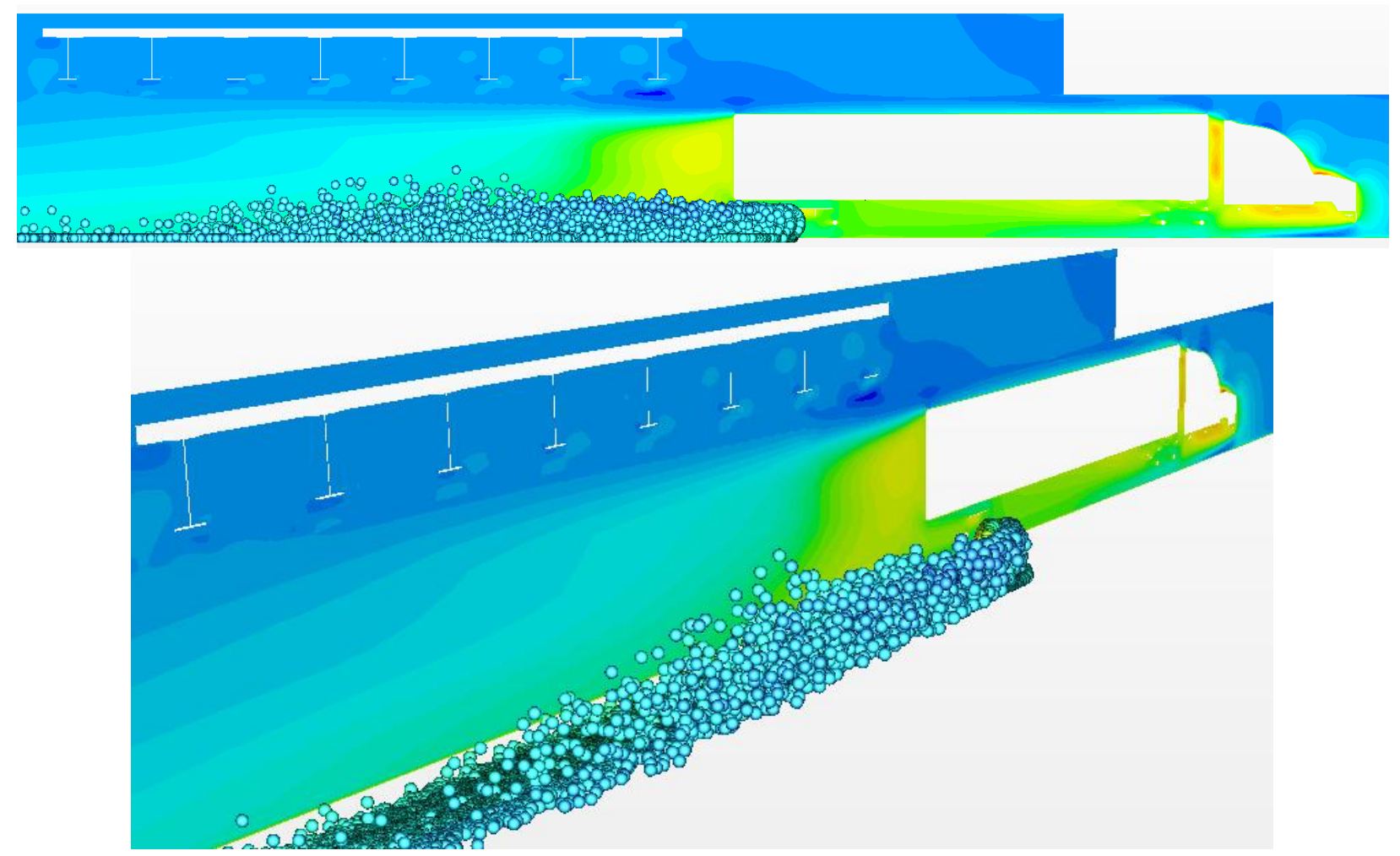

Figure 8.56: Velocity field around the truck and distribution of 50 micron droplets injected at the rear tire tread

Also to the side at the angle of 45 degrees, droplets with a diameter of 100 microns were injected. Figure 8.57 shows results of this simulation. Here the effect of air moving in the wake of the truck on droplets is negligible. Once the droplets leave the boundary layer of air moving with the truck, they decelerate quickly to the zero ambient wind speed in the simulation and settle to the ground under the influence of gravity. 

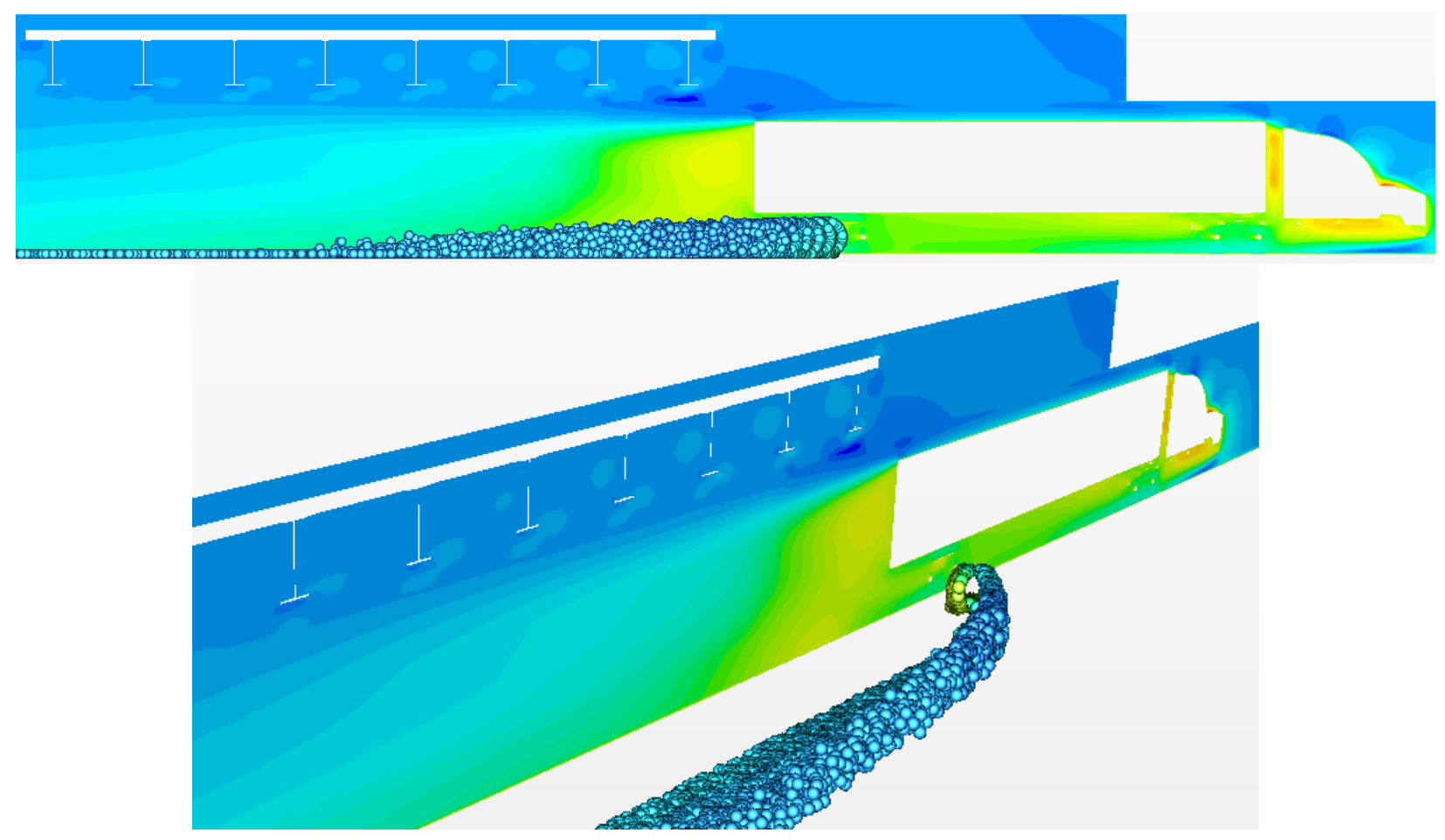

Figure 8.57: Velocity field around the truck and distribution of 100 micron droplets injected at the rear tire tread

\subsection{Droplets with mass released on from the tires at the angle of $45 \mathrm{deg}$}

The behavior of 50 micron droplets was also tested in a case where they were injected from the rear tire at the angle of 45 degrees in respect to the ground, determined by vector $(-1.0,-0.1,1.0)$. In this case the droplets were entering the air behind the vehicle with no mud flaps (a worst case scenario) that was traveling with the speed close to the vehicle velocity. Figure 8.58 shows the trajectories of these droplets. The droplets reach a maximum of about $2 / 3$ of the trailer's height. Even in this worst case without the mud flaps, the droplets were not reaching the height of the beams in the bridge. 

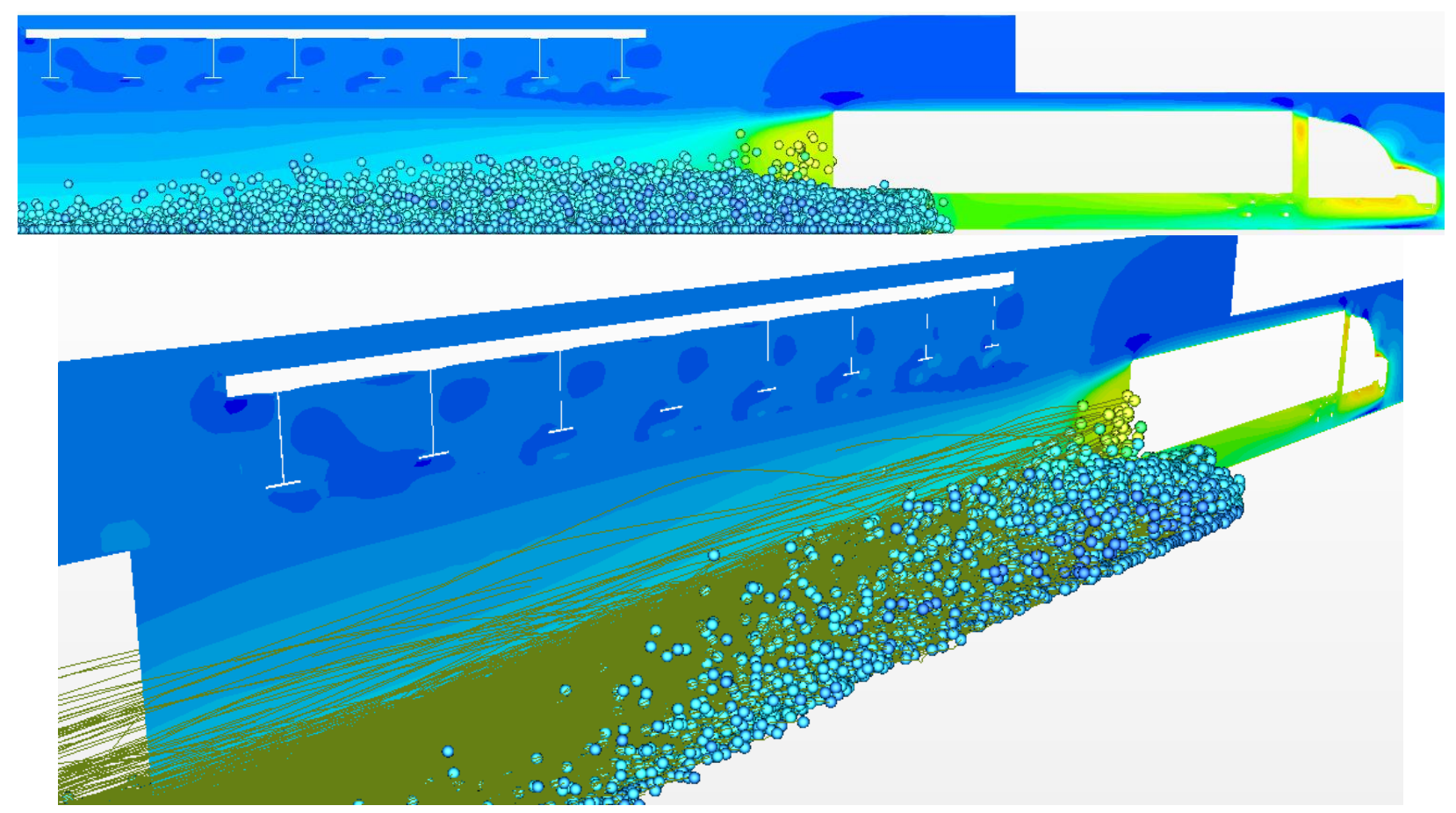

Figure 8.58: Velocity field around the truck and distribution of 50 micron droplets injected at the rear tire tread

A similar test was conducted for droplets with the diameter of 100 microns. Figure 8.59 shows the droplet distribution when the vehicle is leaving the bridge area. A nearly ballistic trajectory of droplets that reach maximum height can be seen. This case is also a worst case trial and not representative of normal conditions because the angle of injection is high and mud flaps are not present in the model. However, even in this extreme case, virtually no droplets are reaching the bridge beams.

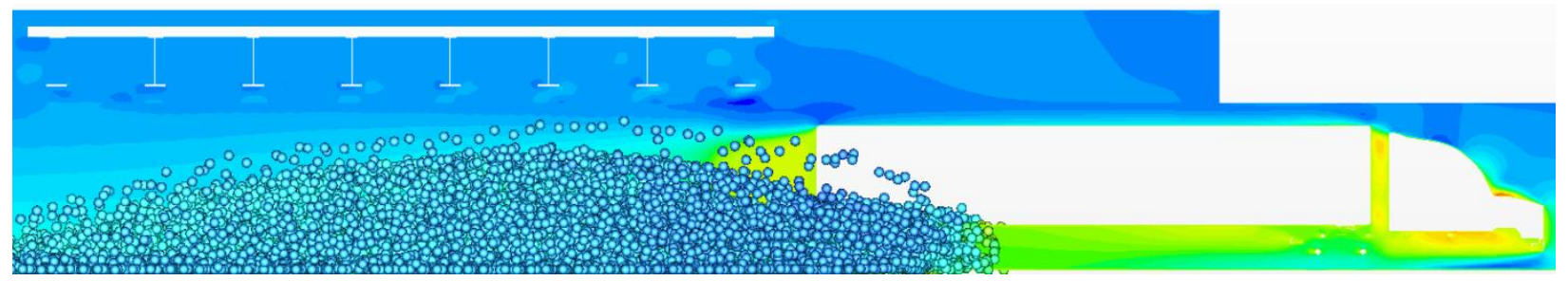

Figure 8.59: Velocity field around the truck and distribution of 100 micron droplets injected at the rear tire tread

\subsection{Mass droplets released on from the tires at the angle of $15 \mathrm{deg}$}

Based on the analysis conducted in [2] and observations of real trucks during rain storms, the angle of injection of droplets from underneath the wheel well and mud flaps is most realistic at angles less than about 15 degrees. Droplets with the diameter of 100 microns were selected for this analysis. Although this is a fairly large uncertainty in injection rates, size distribution, and injection angle, this case is closest to reality based on current knowledge and available data. Treads on all the tires on one side of the truck were selected as the injectors. As seen in Figure 8.60, droplets from the front tire are leaving the wheel well through the side although the initial angle of injection was in the vertical plane of vehicle travel (X-Y plane). This behavior has been noted in several observations of the production of spray from trucks on 
the road during rains. Many droplets are lifted behind the truck and traveling with it. However, none of them reaches the level of the bridge beams.

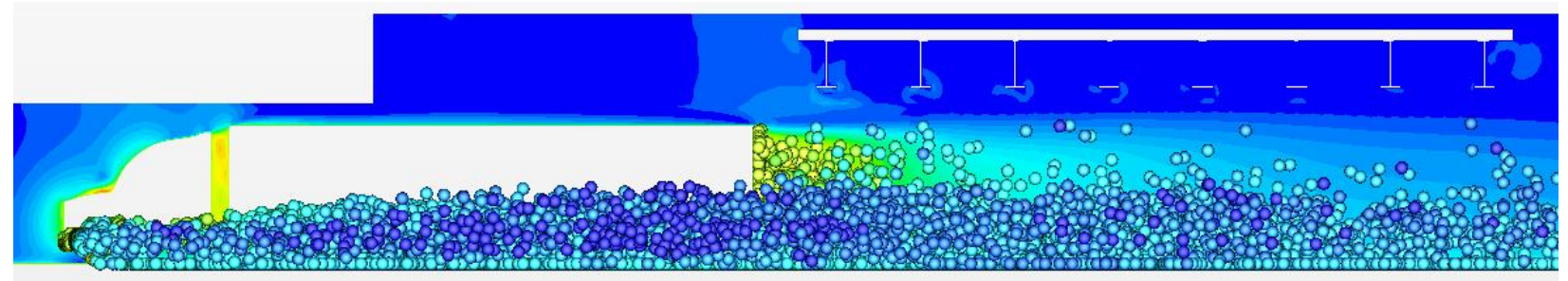

Figure 8.60: Velocity field around the truck and distribution of 100 micron droplets injected at all tire treads on one side of the vehicle

Figure 8.61 shows a picture of a truck traveling on a wet road. The spray around trailer tires and in the wake appears to be a fine mist qualitatively consistent with the droplet breakup modeling in Section 1.3.4.1.2. The visible part of the spray does not reach more than $2 / 3$ of the trailer's height, which resembles the results presented in Figure 8.60.

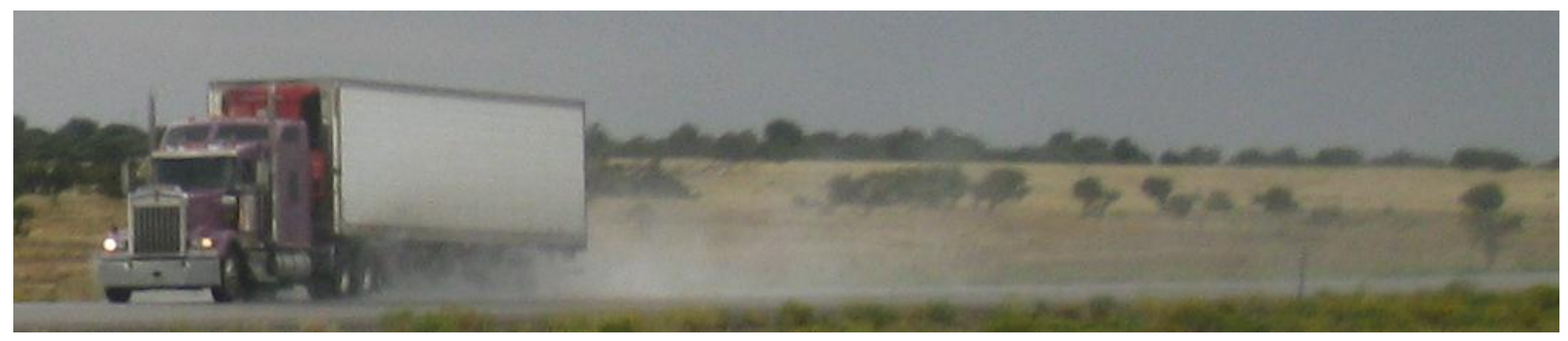

Figure 8.61: Spray behind the truck driving on a wet road

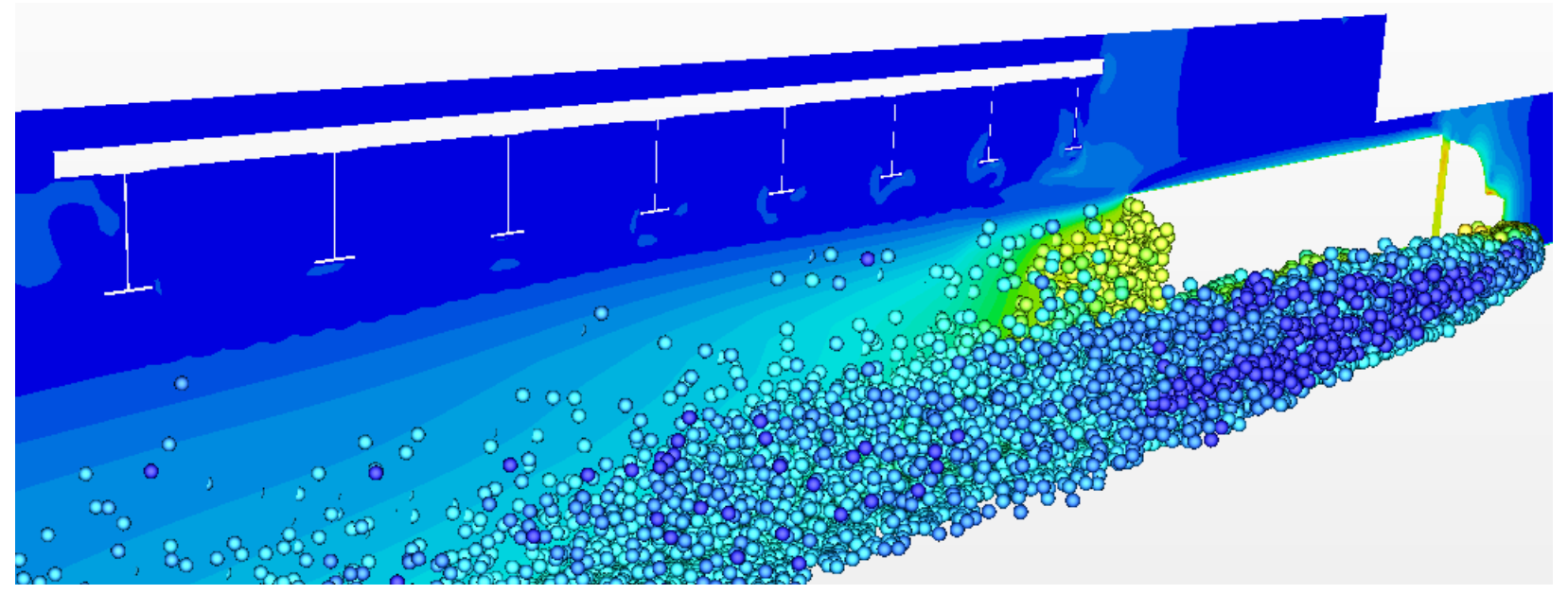

Figure 8.62: Velocity field around the truck and distribution of 100 micron droplets injected at all tire treads on one side of the vehicle

As shown in Figure 8.62 the droplets are leaving the wheel well area to the side of it (although the injection vector does not have a $Y$ component $(-1.0,0.0,0.268)$ ). Similar behavior can also be observed in the real world. The pictures in Figure 8.63 show water droplet spray leaving the partially closed cavity around the wheels created by the mud flaps, the bottom of the trailer, and the back of the cab as a fine mist. The portion leaving from under the mud flaps moves primarily rearward, but the portion leaving 
from the top of the tires has rearward flow blocked by the mud flaps and must move out to the side of the truck.

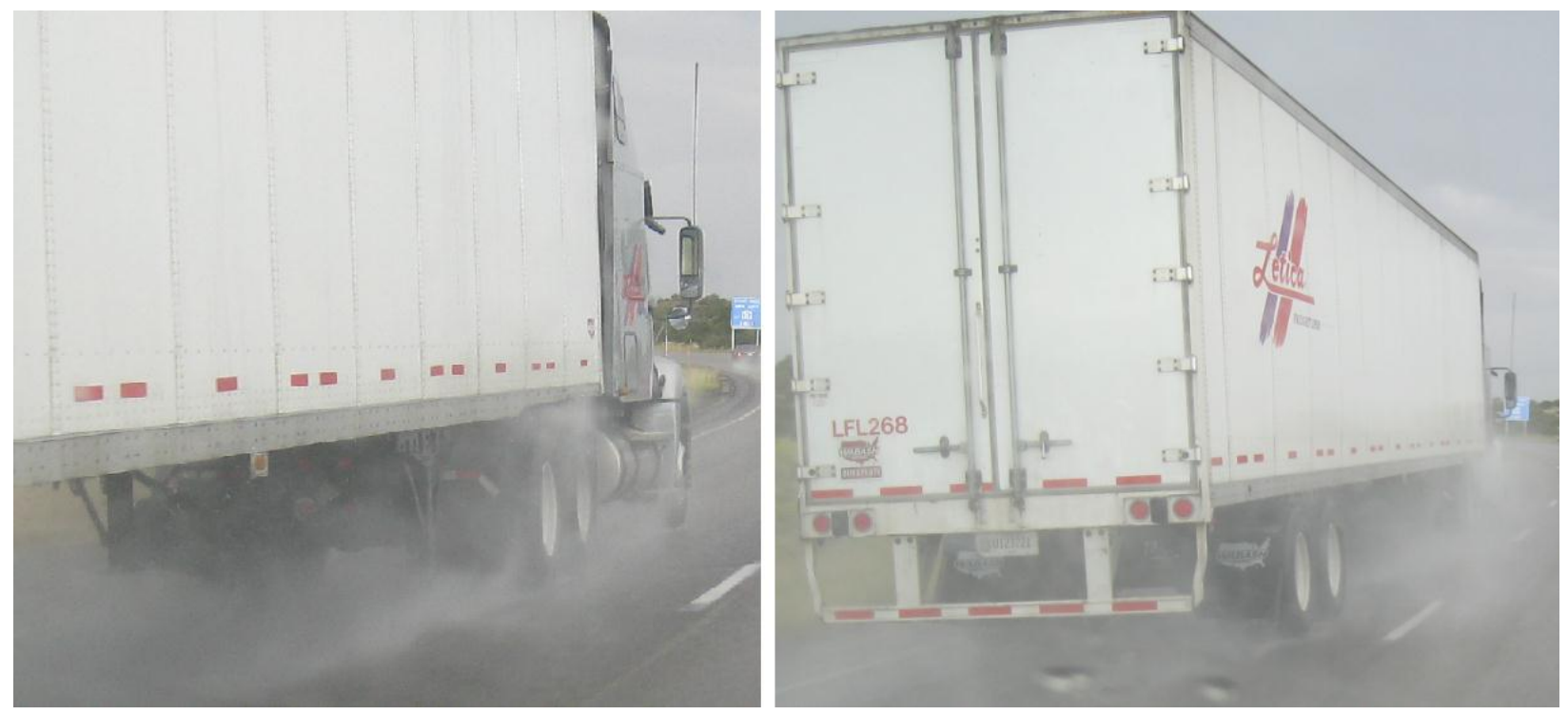

Figure 8.63: Generation of the fine spray mist on the side of the truck

From the simulations conducted so far it appears that the presence of the bridge deck and beams has little influence on the behavior of the droplets in the water spray generated by a truck moving at 26.8 $\mathrm{m} / \mathrm{s}(60 \mathrm{mph}$ ). Another, possibly more important factor is the presence of obstacles on the side of the traveling vehicle. Although the bridges listed by TFHRC as affected by excessive corrosion have open space on the sides, the presence of walls on the sides is expected to influence the air flow pattern on the side and in the wake of trucks, which can affect droplet transport through drag. To test the effect of vertical bridge walls near the roadway, wall boundary conditions were applied on the interface between the truck box and the bridge domain. Figure 8.64 on the top shows the droplet distribution just as the truck is leaving the zone under the bridge with a vertical wall. Comparing to the case without a vertical wall shown in Figure 8.64, there are more droplets up near the bridge beams at about 5 seconds into the simulation. However, the simulation needs to be continued after the truck exits the zone under the bridge to determine if significant numbers of droplets hit the beams of the bridge. Figure 8.64 on the bottom shows droplets hitting the beams after 10 seconds of simulation. Figure 8.65 shows the history of particle hits per time step $(0.02 \mathrm{~s})$. There are 20 iterations per time step. The first droplet hit occurred at about 6.7 seconds and the last ones at about 9 seconds. Cumulatively no more than 50 droplets hit the beams of the bridge. This is a very small number considering the fact that each time step (0.02 sec) about 1300 droplets is injected to the system. A total of 650,000 droplets were injected into the system and only 50 hit the beams for a case where the truck had no mud flaps. The percentage of droplets in the drop wake spray that hit beams when a single truck passes under a bridge with no traffic appears to be negligibly small for the case with a vertical wall. 

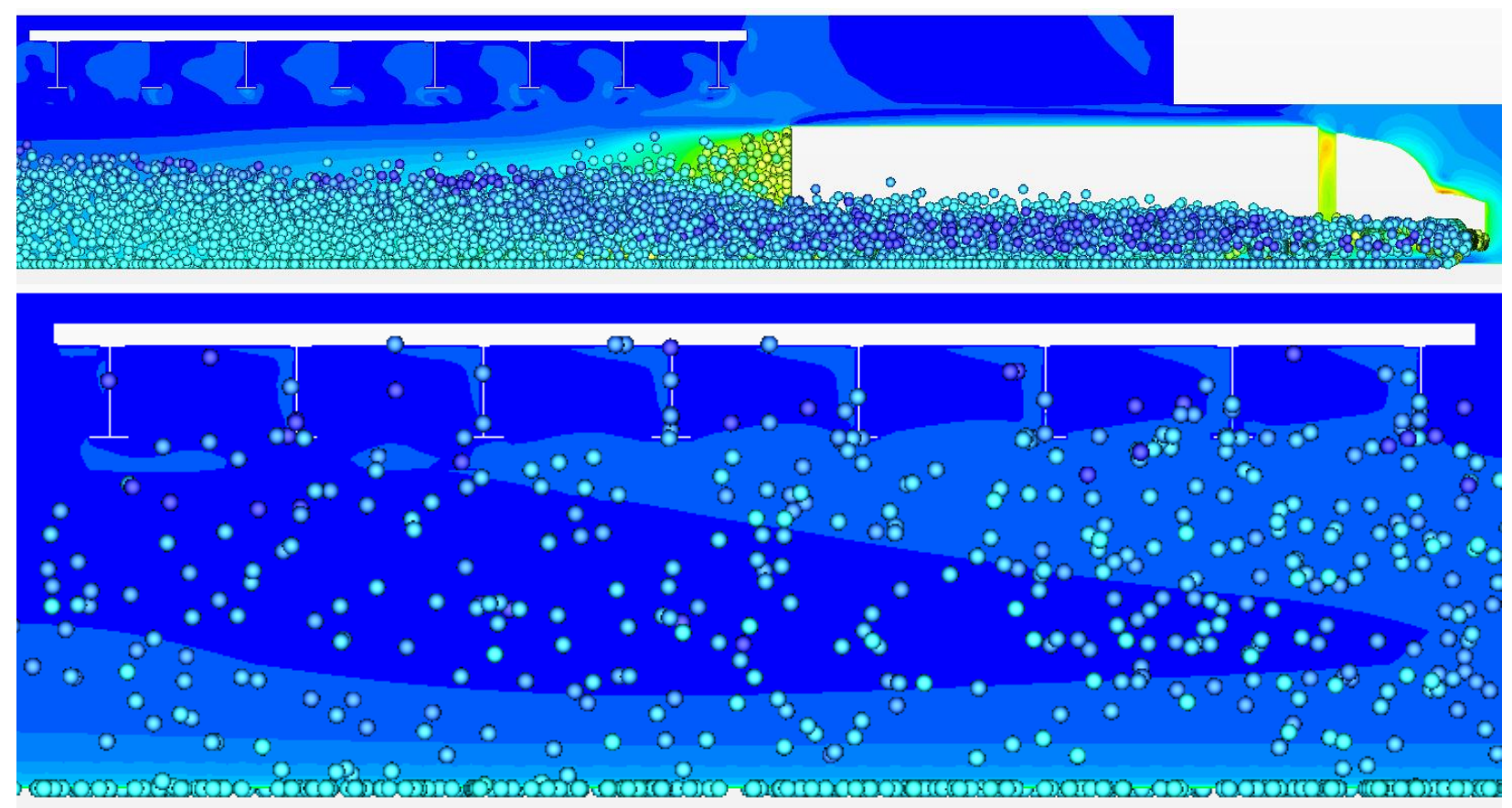

Figure 8.64: Velocity field around the truck and distribution of 100 micron droplets injected at all tire treads on one side of the vehicle

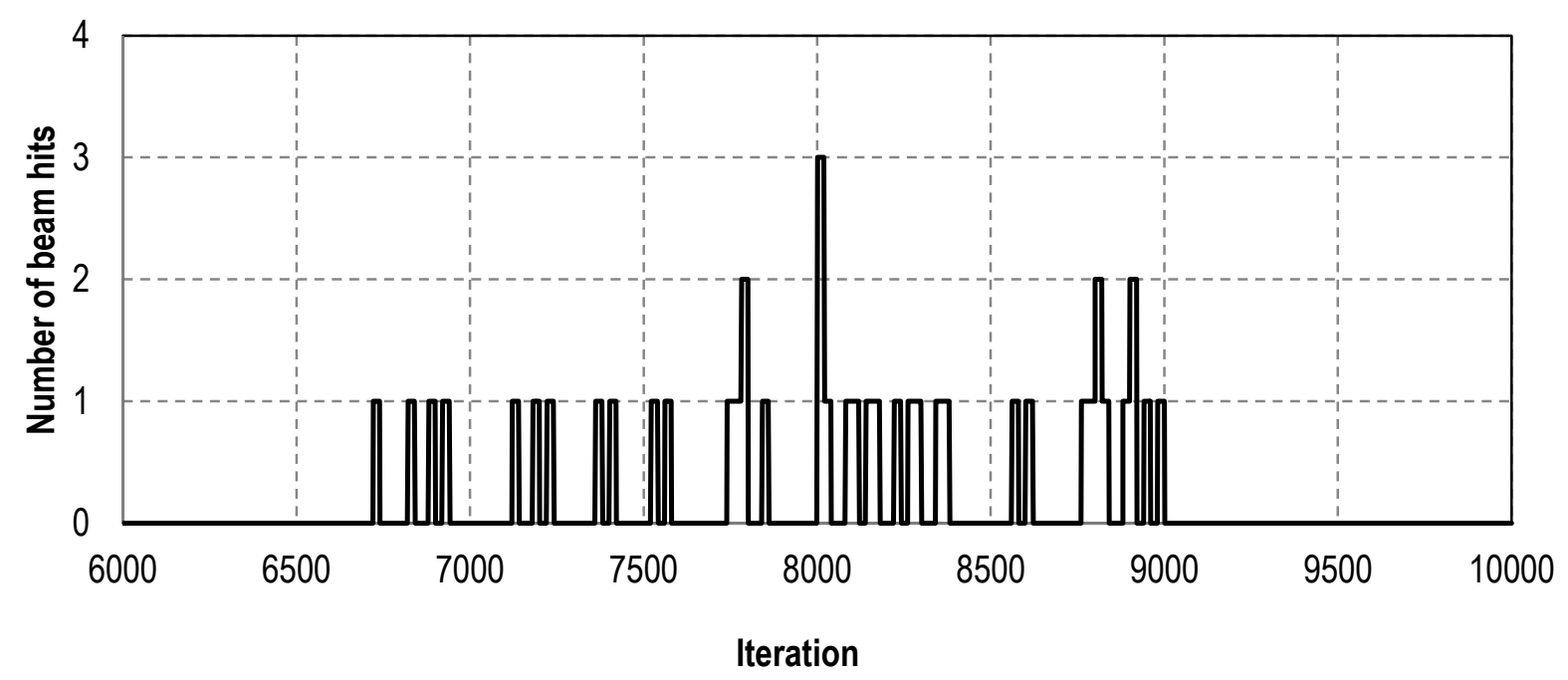

Figure 8.65: Count of droplets hitting the bridge beams during the simulation time

\subsection{Planned simulations}

As the initial runs did not indicate significant transport of droplets to the level of the bridge beams it was decided that subsequent runs should involve other factors that may influence the behavior of the droplets. Three major factors are:

1) presence of other vehicles on the road 
2) additional wind gusts moving the droplets up higher

3) resuspension of droplets and dry road salt as an aerosol by traffic hours to days after salting the road.

Figure 8.66 shows the geometry of the model with two trucks that is being currently analyzed. The results from this simulation and others will be presented in the next report.

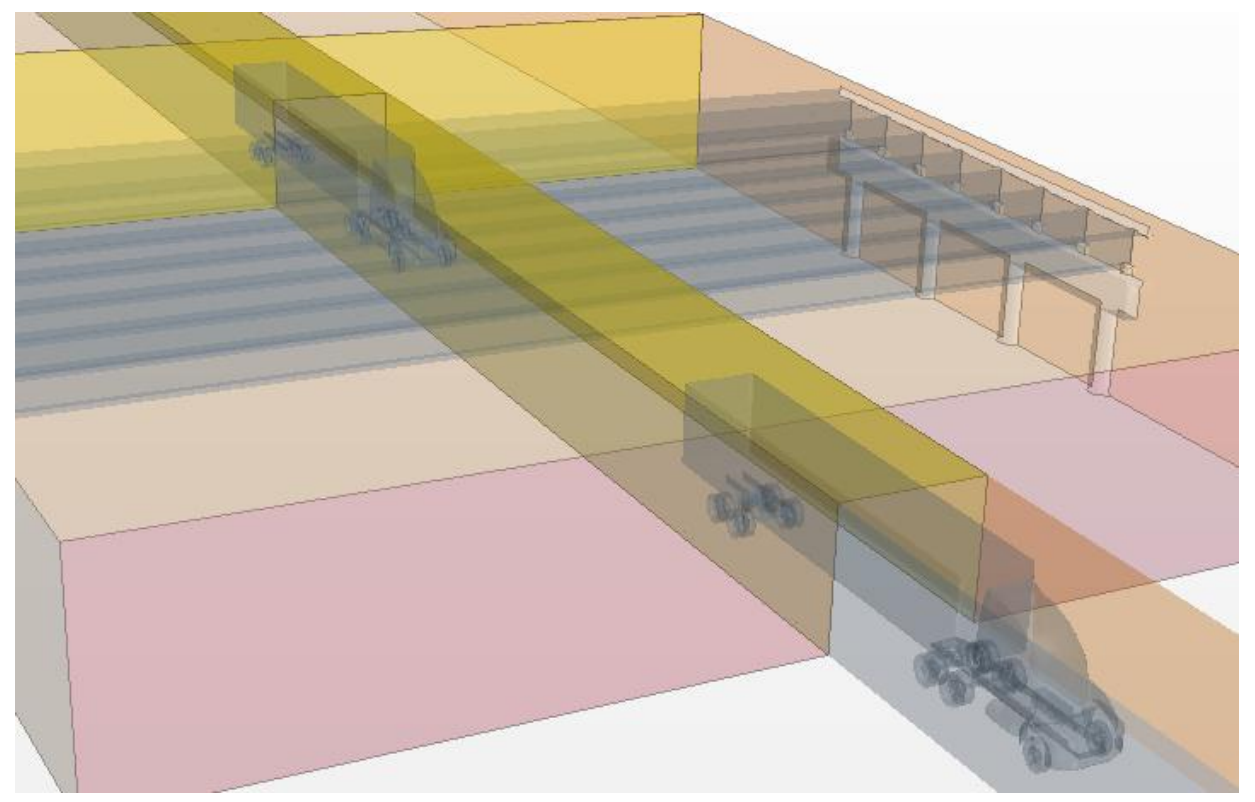

Figure 8.66: Geometry of the model with two trucks

\subsubsection{References}

[1] CD-adapco, User Guide STAR-CCM+ Version 6.02.008, 2011

[2] Ch.A. Radovich, Experiments of spray from a rolling tire, PhD Thesis, University of Southern California, August, 2010

[3] C.X. Bai, H. Rusche, A.D. Gosman, Modeling of Gasoline Spray Impingement, Department of Mechanical Engineering Imperial College of Science, Technology and Medicine, Atomization and Sprays (2002), Vol 12, Issue 1-3, pp 1-28

[4] J.S. Paschkewitz, Simulation of spray dispersion in a simplified heavy vehicle wake, Lawrence Livermore National Lab report No. UCRL-TR-218207, January 17, 2006 


\subsection{Computational Multiphysics Mechanics}

\subsubsection{Multiphysics Simulation of Salt Spray Transport}

\subsubsection{Wind Engineering}

\subsubsection{Vehicle Stability under High Wind Loading}

Ground vehicles, such as automobiles, trucks and trains, are often subjected to crosswinds that can lead to vehicle instability and, in the worst case, vehicle rollover. The current state-of-the-art for performing nonlinear transient analysis of vehicles opens the door to investigate this important and complex phenomenon of wind-structure interaction. At TRACC current capabilities exist to perform coupled wind-structure interaction on a high performance computer, and this should be a long range goal for this project. However, before embarking on that path, it is always prudent to approach the problem in an incremental fashion, i.e., with a series of increasing more complex models. By doing this, a greater understanding of the relative importance of the effect of different physics and a high confidence in the final results will be achieved. This reporting period describes a simplified approach in which the wind is treated as a pressure load on the side of the vehicle. Future work will moved toward doing a multiphysics approach, i.e., coupled fluid-structure interaction.

For the first group of numerical simulations, a fairly complex model for an 8000 s vehicle class truck was used. The following vehicles satisfy the requirements of the $8000 \mathrm{~S}$ vehicle class: Ford, GMC, Chevrolet, Freightliner, and International. Instead of doing a coupled Computational Structural MechanicsComputational Fluid Dynamics analysis, the wind was modeled as a constant pressure load on the windward side of the truck. The following sections provide details and some preliminary results.

\subsection{Cargo Loading, Wind Loading, Road Condition and Vehicle Velocity :}

\section{Cargo Loading}

A complex finite element model for the $8000 \mathrm{~S}$ vehicle was downloaded from the model repository at the National Crash Analysis Center (NCAC) [1]. The model was for a Ford F-800 Single Unit Truck (SUT). This SUT is designed with two-axels, a rear wheel drive and a van type cargo body. It should be noted that this model was developed for simulating SUT crashes into roadside barriers, and thus, modeling features related to that type of analysis were included in great detail. Specifically, the truck model was developed to be used in simulations of Test Level 4 impacts and was to represent an 8000 S test vehicle. Unfortunately, some of the features needed for crosswind loading were not properly accounted for and, thus, were added for wind loading analysis. In addition, because the physical SUT used in the barrier crash tests is not a Ford F-800, ballast mass was added in the cargo body of the model to make up the difference.

The recommended properties for $8000 \mathrm{~S}$ test vehicles are as follows (Table 8.6): curb mass equal to 5.45 tonnes; ballast mass as needed; test inertia (gross vehicle mass equal to 8.00 tonnes. A complete description of the finite element model is given in [2]. The modeled mass of the truck was 5.270 tonne and the ballast mass was 2.872 tonne giving a total mass of 8.142 tonne. It is seen that the model for the Ford F800 truck meets the recommended properties of for an 8000 S test vehicle. 
However, it is important to note that a quick check on the internet [3] reveals differences between a typical Ford F800 and the Ford F800 finite element model used to represent the $8000 \mathrm{~S}$ test vehicle. For example, for a typical F800 truck the following data (Table 8.6) was found: curb mass equal to 3.62 tonne ( $66 \%$ of the model); gross vehicle mass equal to 11.8 tonne ( $48 \%$ larger than the model); and payload equal to 8.174 tonne, (3.2 times larger than the ballast).

Table 8.6: Comparison of finite element model for a SUT to a Ford F800 truck.

\begin{tabular}{|l|l|l|l|l|l|l|}
\hline & Curb Mass & Curb Weight & $\begin{array}{l}\text { Ballast Mass } \\
\text { or Payload }\end{array}$ & $\begin{array}{l}\text { Ballast } \\
\text { Weight or } \\
\text { Payload }\end{array}$ & $\begin{array}{l}\text { Gross } \\
\text { Vehicle } \\
\text { Mass }\end{array}$ & $\begin{array}{l}\text { Gross } \\
\text { Vehicle } \\
\text { Weight }\end{array}$ \\
\hline $\begin{array}{l}\text { NCAC } \\
\text { Finite } \\
\text { Element } \\
\text { Model [1] }\end{array}$ & 5.45 tonne & $53,000 \mathrm{~N}$ & 2.55 tonne & $25,000 \mathrm{~N}$ & 8 tonne & $78,000 \mathrm{~N}$ \\
\hline $\begin{array}{l}\text { Ford F800 } \\
\text { Truck [3] }\end{array}$ & 3.62 tonne & $35,490 \mathrm{~N}$ & 8.174 tonne & $80,160 \mathrm{~N}$ & 11.8 tonne & $115,700 \mathrm{~N}$ \\
\hline
\end{tabular}

In the following work, the NCAC model had to be used because of the lack of a finite element model for the heavier F800. For this initial study, the cargo was represented by a fixed (in size and location) rectilinear mass inside the cargo body (Figure 8.67). This mass was rightfully referred to as ballast in [2], but in this study it represents cargo and will be referred to as such. To study the effect of different cargo weights, the density of the cargo material was varied as shown in Table 8.7. The cargo mass affects the position of the center of gravity (COG), which is a crucial factor to estimate rollover. However for this initial study, the COG for the cargo mass is fixed because the cargo is fixed in size and location, but the COG for the combined truck and cargo will vary as the density of the cargo changes.

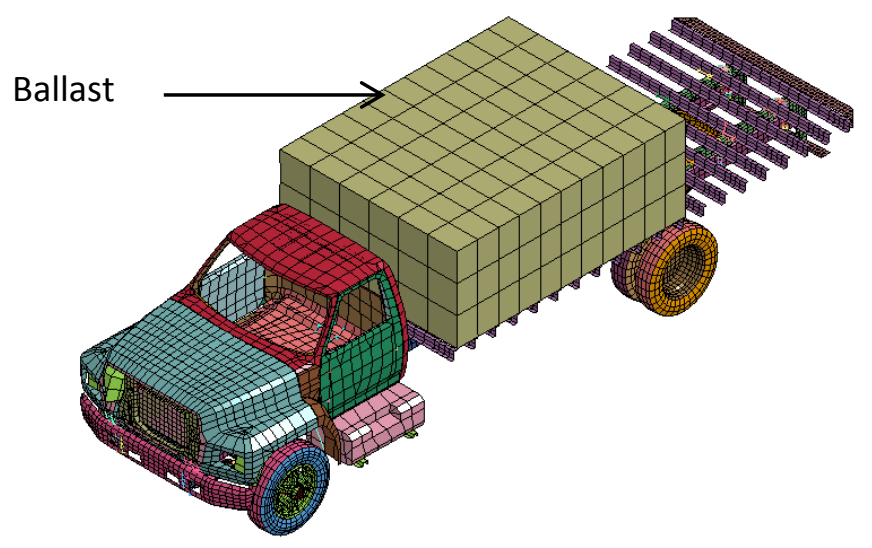

Figure 8.67: Ballast portion [1] 
Table 8.7: Cargo loads

\begin{tabular}{|c|c|c|c|}
\hline \multirow{2}{*}{ Cargo } & \multicolumn{3}{|c|}{ Load Condition } \\
\cline { 2 - 4 } & $\mathbf{1 0 0 \%}$ Mass & $\mathbf{5 0 \%}$ Mass & $\mathbf{1 0 \%}$ Mass \\
\hline $\begin{array}{c}\text { Density } \\
\text { (tonne/mm }\end{array}$ & $3.136 \times 10^{-10}$ & $1.568 \times 10^{-10}$ & $3.136 \times 10^{-11}$ \\
\hline Mass (tonne) & 2.80938 & 1.40469 & 0.280938 \\
\hline
\end{tabular}

\section{Wind Loading}

As a truck travels down a highway under no wind conditions, it is subjected to aerodynamic loading along the length of the truck because of vehicle speed - a sort of pseudo wind. In addition, the truck can be subjected to aerodynamic loading because of crosswinds. Records show that horizontal wind speed varies with time. Within intervals of about 300 seconds, wind speed history records [4] show that wind gusts occur about a mean wind speed. For this preliminary analysis, it is assumed that aerodynamic loading across the SUT is considered to be equal to a horizontal crosswind with a constant speed. Most of the simulations reported here are for about 5 seconds; so this assumption is reasonable. It is further assumed that the crosswind speed does not vary with the height of the vehicle, which is not exactly true since the wind speed at ground level is zero. The across-the-truck aerodynamic loading is applied to the driver side of the truck. As described in the last Quarterly Report [5], the pressure applied to the side of the truck was obtained by multiplying the stagnation pressure, which is a function of the wind speed, by the drag coefficient. The value for the drag coefficient was taken to be 1.0. It is noted that a drag coefficient specific to the F-800 could not be located.

The Beaufort Wind Scale (Table 8.8) provides a correlation of wind speed with quantitative and qualitative descriptions that are identified by their Beaufort Number (BN). Table 8.8 shows that gale winds begin at $32 \mathrm{mph}(\mathrm{BN}=7)$ and end at $54 \mathrm{mph}(\mathrm{BN}=9)$. The storm category is defined by $\mathrm{BN}=10$ and $B N=11$, and the wind range is from $55 \mathrm{mph}$ to $72 \mathrm{mph}$. The hurricane category is $B N=12$ and the winds are $73 \mathrm{mph}$ and higher.

Table 8.8: Beaufort Wind

\begin{tabular}{|c|c|l|}
\hline $\begin{array}{c}\text { Beaufort } \\
\text { Number }\end{array}$ & $\begin{array}{c}\text { Wind speed } \\
\text { (MPH) }\end{array}$ & \multicolumn{1}{|c|}{ Description } \\
\hline 0 & $<1$ & Still, calm air \\
\hline 1 & $1-3$ & Light Air \\
\hline 2 & $4-7$ & Light Breeze \\
\hline 3 & $8-12$ & Gentle Breeze \\
\hline 4 & $13-18$ & Moderate Breeze \\
\hline 5 & $19-24$ & Fresh Breeze \\
\hline 6 & $25-31$ & Strong Breeze \\
\hline 7 & $32-38$ & Moderate or Near Gale \\
\hline
\end{tabular}




\begin{tabular}{|c|c|l|}
\hline 8 & $39-46$ & Gale or Fresh Gale \\
\hline 9 & $47-54$ & Strong Gale \\
\hline 10 & $55-63$ & Whole Gale or Storm \\
\hline 11 & $64-72$ & Violent Storm \\
\hline 12 & $73+$ & Hurricane \\
\hline
\end{tabular}

\section{Road Condition}

The road was assumed to be a flat surface. The traction between the tires and road surface affects the response of the truck to wind loading. For this initial analysis, the surface condition of the road was specified by the coefficient of friction (COF) between the tire and road surface. The value of the COF varies between its static and dynamic value. Prior to sliding, the static value governs. During sliding, however, the COF varies as a function of the sliding velocity. In this first analysis, the COF is taken to be constant and equal to the static value as given in Table 8.9 for each road condition. This assumption will be removed in subsequent analyses.

Table 8.9: Coefficient of friction for dry, wet and ice road conditions.

\begin{tabular}{|c|c|c|c|}
\hline Condition & Dry & Wet & Ice \\
\hline COF & 0.9 & 0.5 & 0.2 \\
\hline
\end{tabular}

\section{Vehicle Velocity}

The Ford F800 is a rear wheel drive truck; thus, the velocity of the truck is determined by the rotational speed of the rear wheels and the traction between the wheels and road. The rear wheels move the truck in the direction normal to the rear axle; the front steering wheels determine the direction of travel. Because the downloaded Ford F800 SUT truck model was developed for crash analysis, the velocity at all the nodes within the model are the same, which for crash analysis is the correct initial condition.

The condition for wind loading, however, is more complicated. First, whenever a rear wheel lifts off, the traction from that wheel reduces to zero, and the truck is driven only by the opposite wheel. Second, when the horizontal wind rotates the truck relative to the original direction, the drive wheels will still tend to drive the truck in the direction normal to the rear axle, and the truck driver will try to compensate by steering the truck. Because this work represents a first look at this problem, the crash analysis assumption was retained. This assumption will be removed in subsequent, more complex analyses.

\subsection{Simulation results}

The results from the numerical simulations for the transient dynamic response of a SUT loaded by wind on the driver's side are described here. The general rotational transient behavior of the SUT is that the 
wind-load causes the vehicle to pitch, roll and yaw about the center of mass. Under the right set of conditions, a wheel will lose contact with the ground and liftoff. The time at which a wheel lifts off the pavement can be determined as the time at which the contact force between the wheel and ground becomes zero.

To measure the forces generated by the wheels on the ground surface, each wheel is in contact with a separate rigid wall. A rigid wall is a LS-DYNA feature that can be used to measure contact forces, which for our study is the force between a wheel and the pavement. Studying the contact forces helps understand the weight transfer of the model as it is being influenced by the wind. The wind load is applied to right side of the model, which would cause the right side of the truck to lift off the road surface. Note in Figure 8.68, it appears that the loading is also applied to the cargo, but this is not the case. Simulations show that the driver side rear wheel is the first to liftoff. Eventually, the truck may rollover. The driver side rear wheel (right rear tire) is in contact with rigid wall number 3 of the model, and thus, analyzing the force on this rigid wall would let us know the exact time of liftoff.

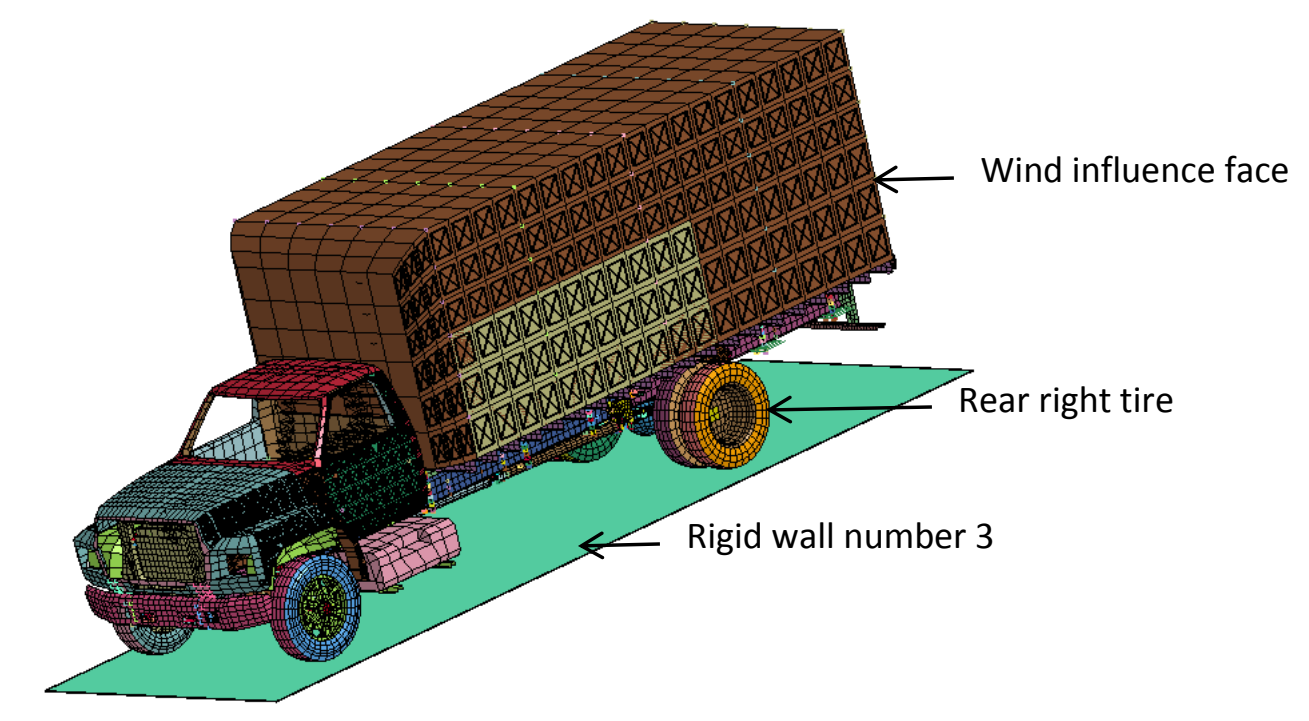

Figure 8.68: Wind influence face and rigid-wall attached with right wheel [1]

Figure 8.69 shows the driver-side rear wheel just after liftoff. In the extreme case, the SUT will rollover as shown in Figure 8.70. The remainder of this section describes preliminary results obtained using the SUT model described above. 


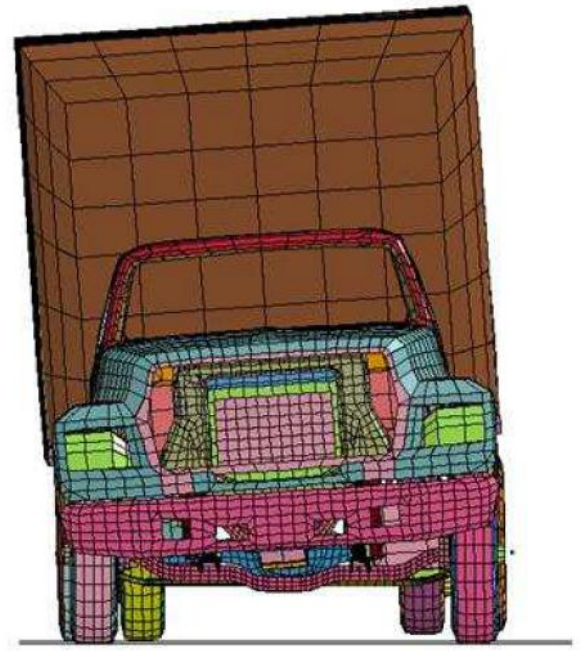

Figure 8.69: Configuration of SUT with driver side rear wheel liftoff.

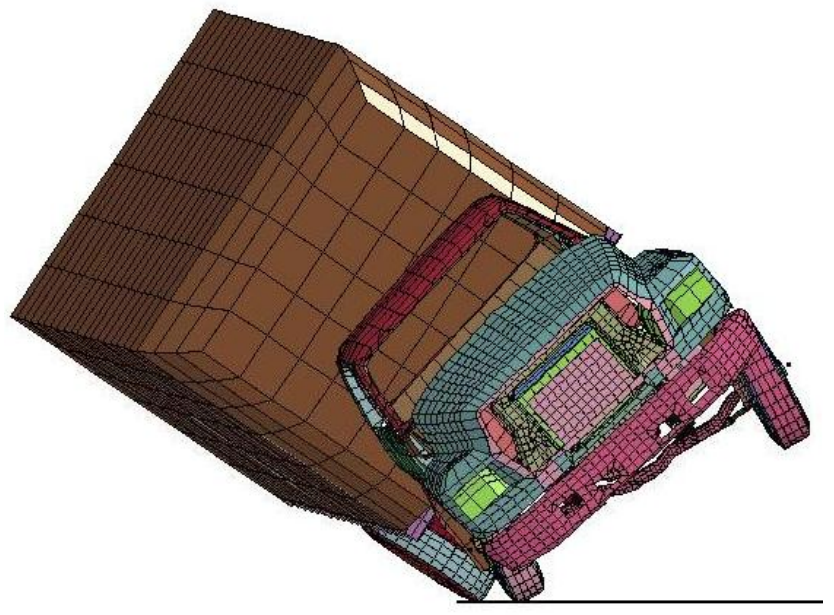

Figure 8.70: Configuration of SUT in the process of rolling over.

\section{Critical driving speeds for vehicle rollover and side slip}

The rollover response of the model with different cargo loading conditions and different coefficients of friction (COF) between the tires and road surface is shown in Figure 8.71 (dry surface, COF $=0.9$ ) and Figure 8.72 (wet surface, COF $=0.5$ ). The vehicle with a $10 \%$ cargo load will roll over at lower speeds than the fully loaded ( $100 \%$ cargo) for all speeds and both the dry and wet road conditions. 


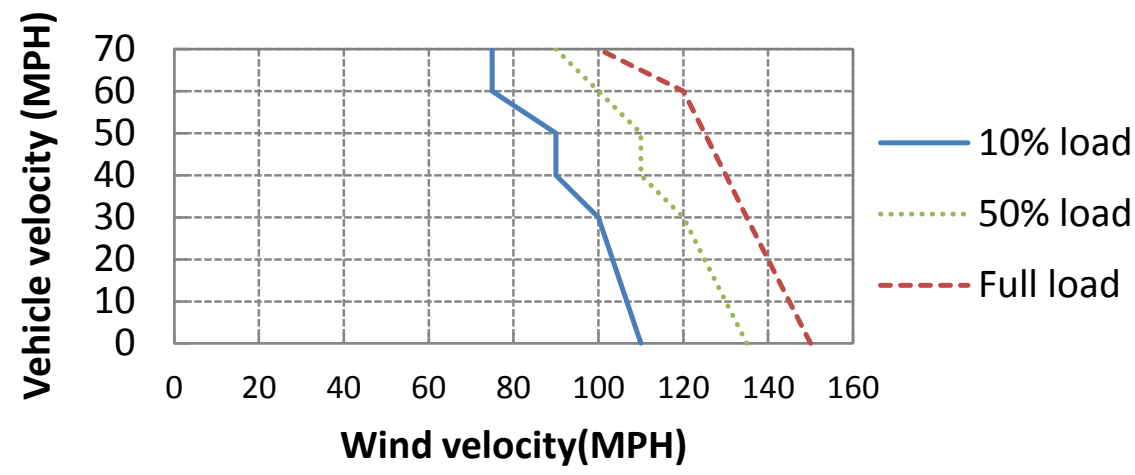

Figure 8.71: Critical driving speed for rollover on dry pavement $(\mu=0.9)$

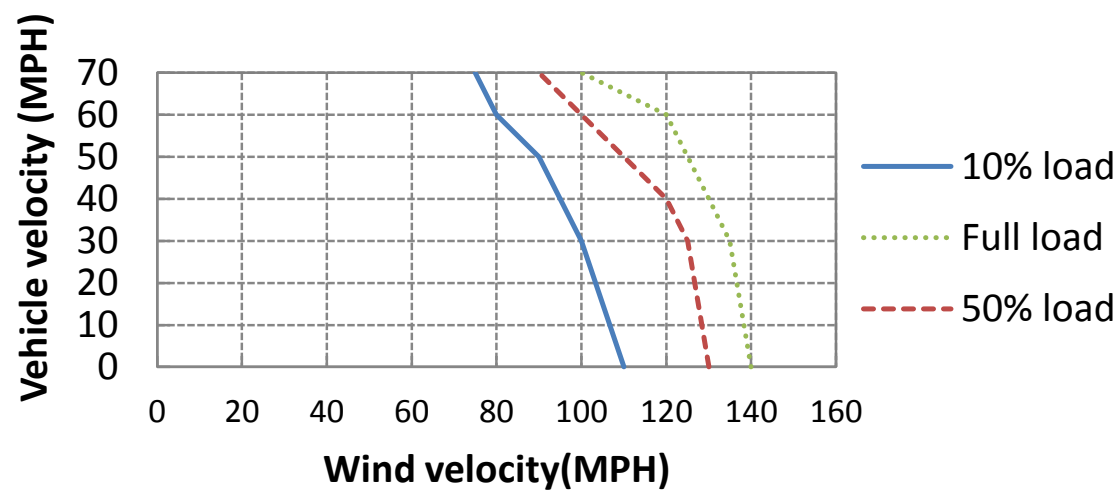

Figure 8.72: Critical driving speed for rollover on wet pavement $(\mu=0.5)$

The graphs of critical driving speeds for sideslip and rollover under different road conditions (i.e., coefficients of friction) and cargo loading are shown from Figure 8.73 to Figure 8.80 . Figure 8.73 to Figure 8.75 illustrates the rollover and sideslip behavior of vehicle travelling on dry road surface (COF = 0.9 ) for full, $50 \%$, and $10 \%$ cargo loading condition. 


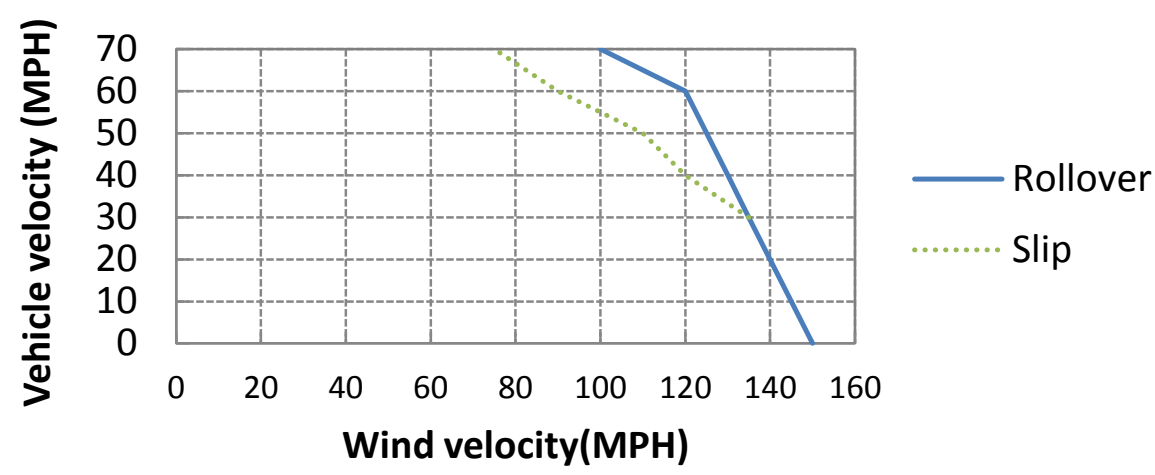

Figure 8.73: Critical driving speed for sideslip and rollover on dry pavement $(\mu=0.9)$ for a truck with a full cargo load.

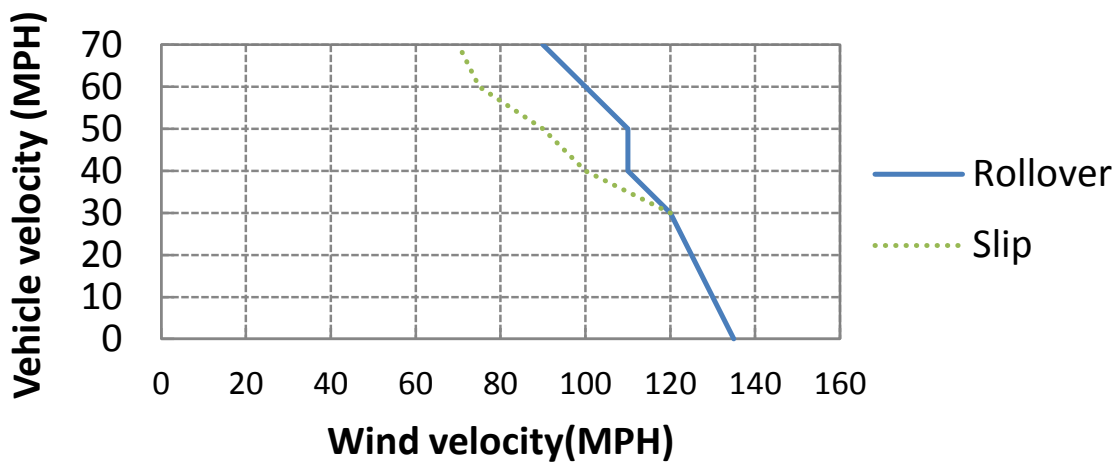

Figure 8.74: Critical driving speed for sideslip and rollover on dry pavement $(\mu=0.9)$ for a truck with a $50 \%$ cargo load

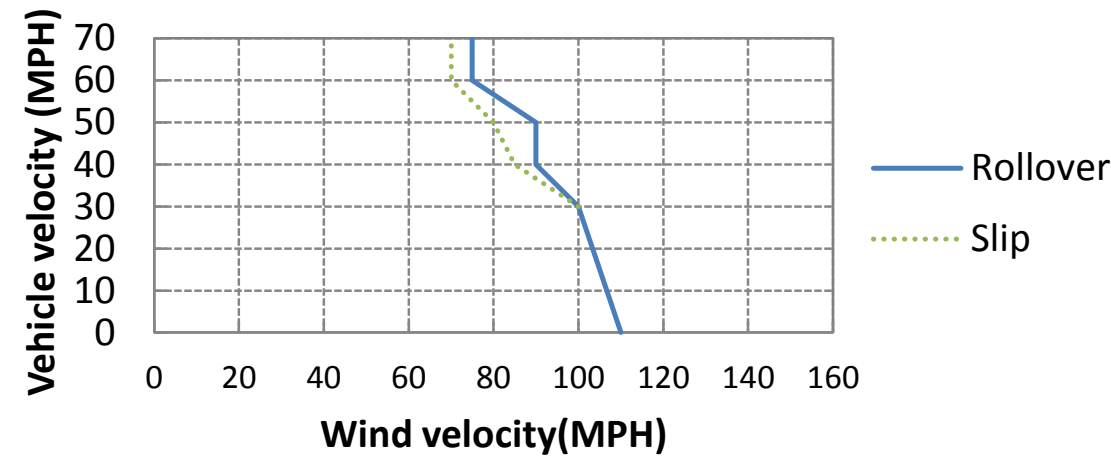

Figure 8.75: Critical driving speed for sideslip and rollover on dry pavement $(\mu=0.9)$ for a truck with $10 \%$ cargo load. 
Figure 8.76 to Figure 8.78 shows the onset of sideslip and rollover of a truck travelling on a wet road surface $(\mathrm{COF}=0.5$ ) with 100 percent, 50 percent and 10 percent cargo loading conditions. 


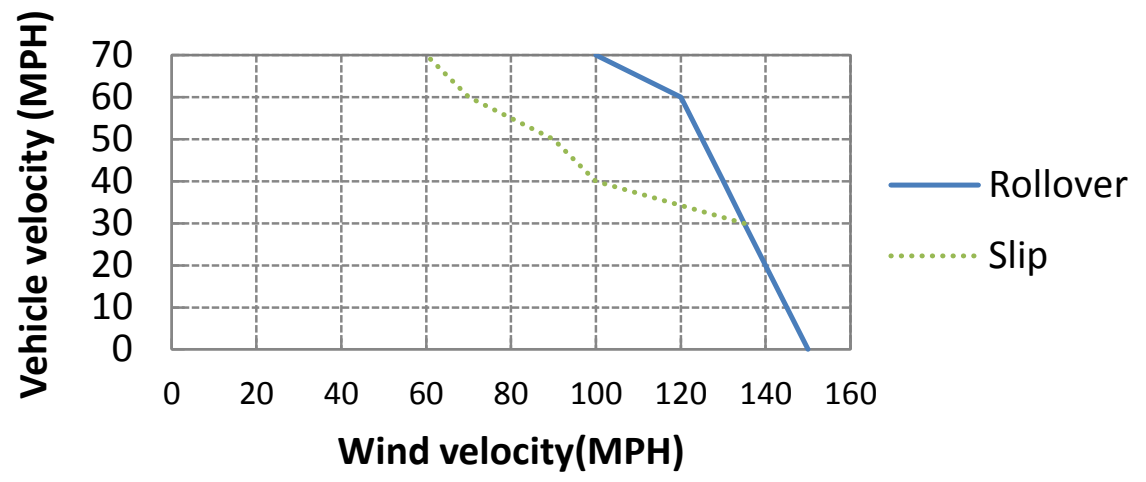

Figure 8.76: Critical driving speed for sideslip and rollover on wet pavement $(\mu=0.5)$ full cargo load.

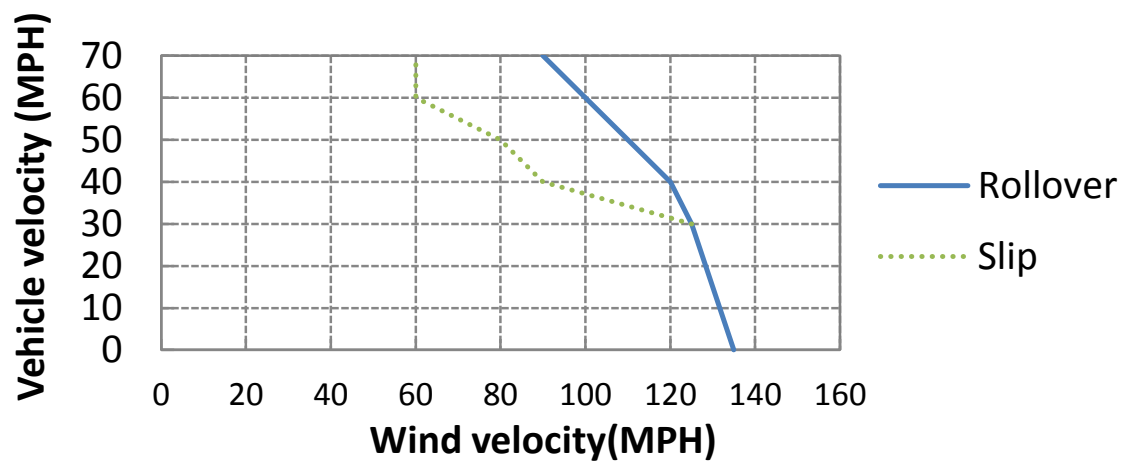

Figure 8.77: Critical driving speed for sideslip and rollover on wet pavement $(\mu=0.5)$ with $50 \%$ cargo load.

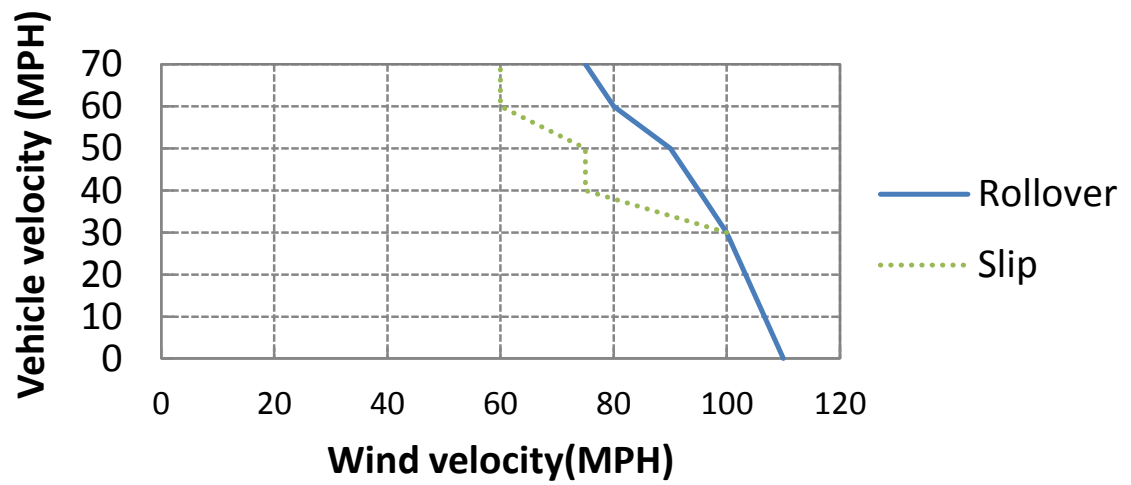

Figure 8.78: Critical driving speed for sideslip and rollover on wet pavement $(\mu=0.5)$ with $10 \%$ cargo load. 
The onset of sideslip and rollover for a truck travelling over an ice covered road surface $(\mathrm{COF}=0.1)$ with full, $50 \%$ and $10 \%$ cargo loading is shown in Figure 8.79 to Figure 8.81 . The simulation results indicate that no rollover will occur for this low value of the COF. However, considering the situation in which the truck reaches the shoulder - which may be a gravel shoulder - the COF could jump from 0.1 to 0.5 , and this could initiate rollover. However, this condition was not simulated here. 


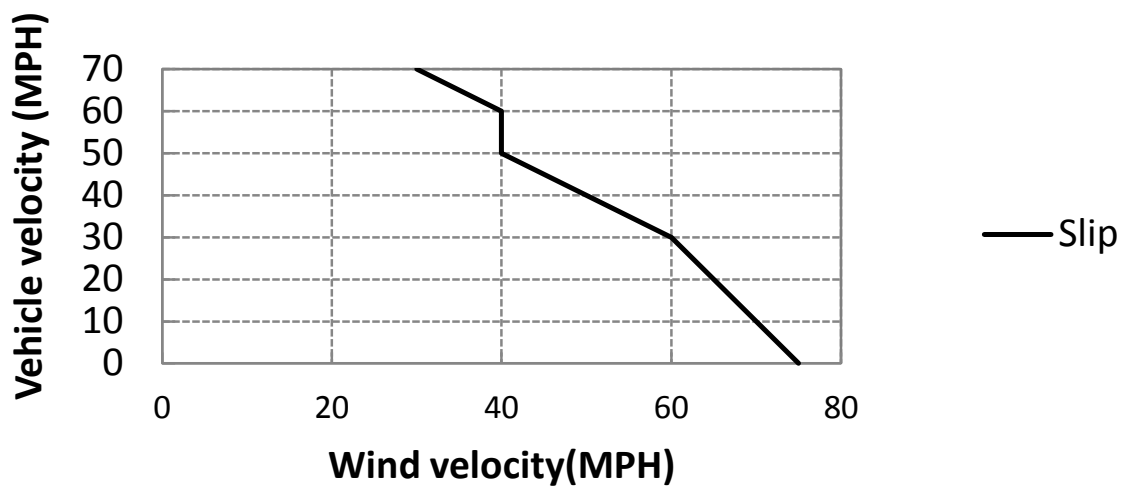

Figure 8.79: Critical driving speed for sideslip on icy pavement ( $\mu=0.1$ ) with full cargo load)

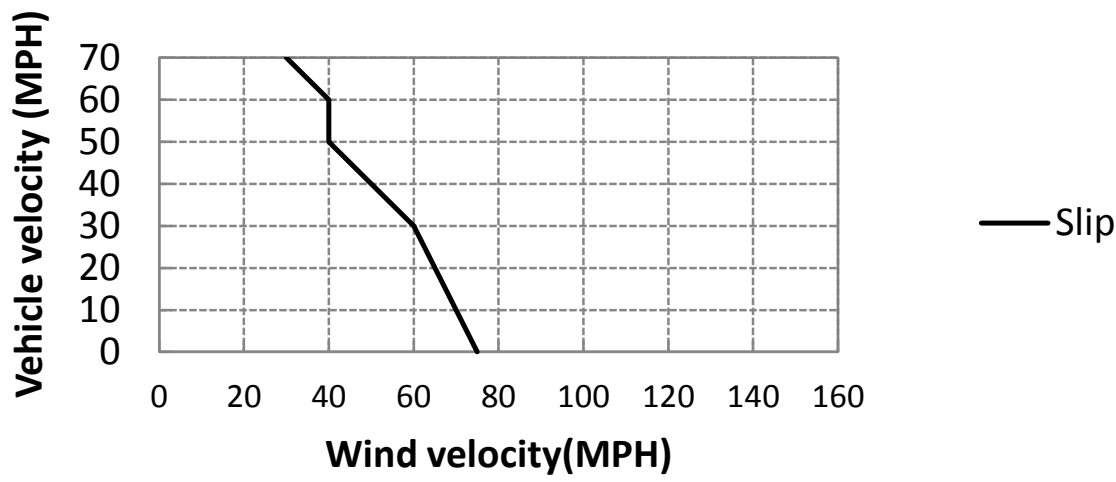

Figure 8.80: Critical driving speed for sideslip on icy pavement $(\mu=0.1)$ with $50 \%$ cargo load

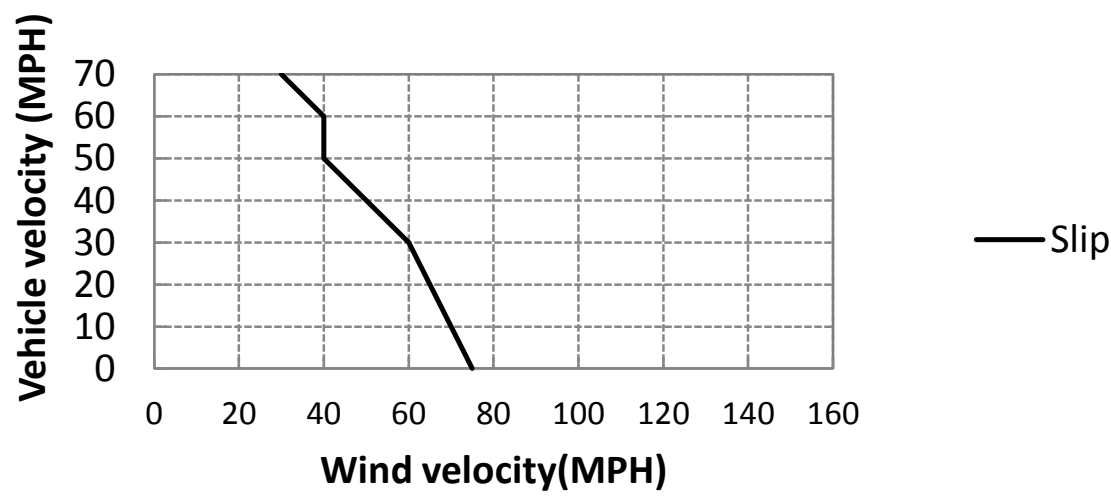

Figure 8.81: Critical driving speed for sideslip on icy payment $(\mu=0.1)$ with $10 \%$ cargo load 


\subsection{Critical sustained time for initial wheel liftoff and vehicle rollover}

Since the wind loading was considered to be constant during each simulation, it is interesting to study the length of time that the wind would have to act for a wheel to lose contact with the ground, i.e., the critical sustained time for initial lift-off. Similarly, the critical sustained time for rollover is of interest. Figure 8.82shows the time history of the contact force for the driver's side rear wheel, which is the first wheel to lift off, when the SUT is subjected to a crosswind of $75 \mathrm{mph}$. First gravity is applied to the vehicle, and static equilibrium is reached at 0.5 seconds with the contact force between the wheel and pavement being $33 \mathrm{kN}$. Next the constant magnitude wind loading is applied to the driver's side of the SUT. It is seen that under the constant wind force, the truck rocks back and forth several times with the wheel lifting off the road until at about $5 \mathrm{sec}$ the wheel lifts off the road and the truck rolls over - recall the wind loading is applied at $0.5 \mathrm{sec}$.

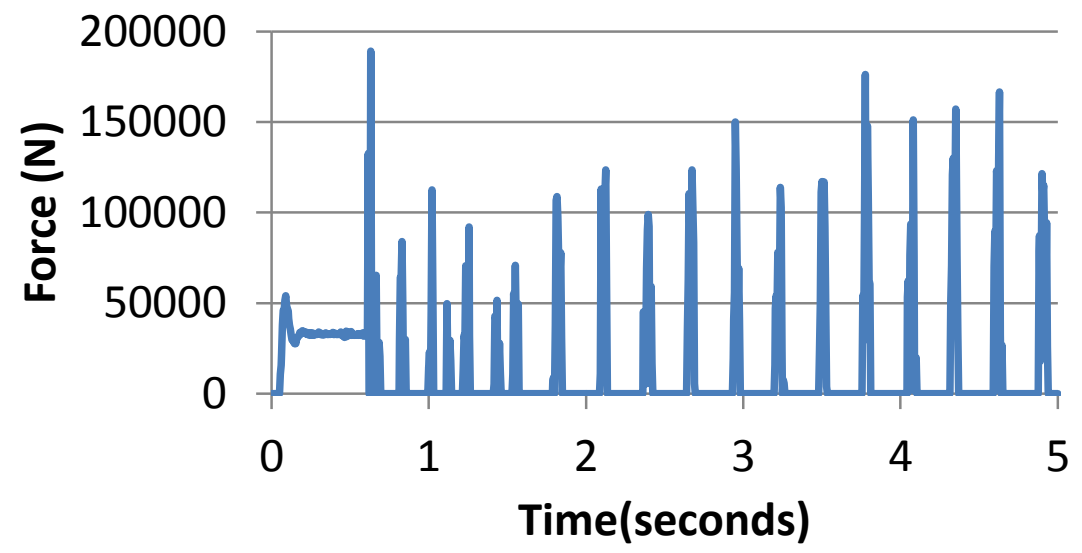

Figure 8.82: Temporal contact force history for driver side rear tire under a $75 \mathrm{mph}$ crosswind

The graphs of critical sustained time - i.e., the minimum length of time under constant wind loading for liftoff and rollover for different coefficients of friction and cargo loading combinations are shown in Figure 8.83 to Figure 8.94 .For the full-load case, Figure 8.83 to Figure 8.84 show the critical sustained times for initial liftoff and the onset of a rollover, respectively. The 0.9 value for the COF represented a dry surface. It is seen that at a vehicle speed of $40 \mathrm{MPH}$, it would take a $75 \mathrm{MPH}$ wind 4.6 sec to cause a liftoff. However, at this vehicle speed and wind speed, no rollover would occur. 


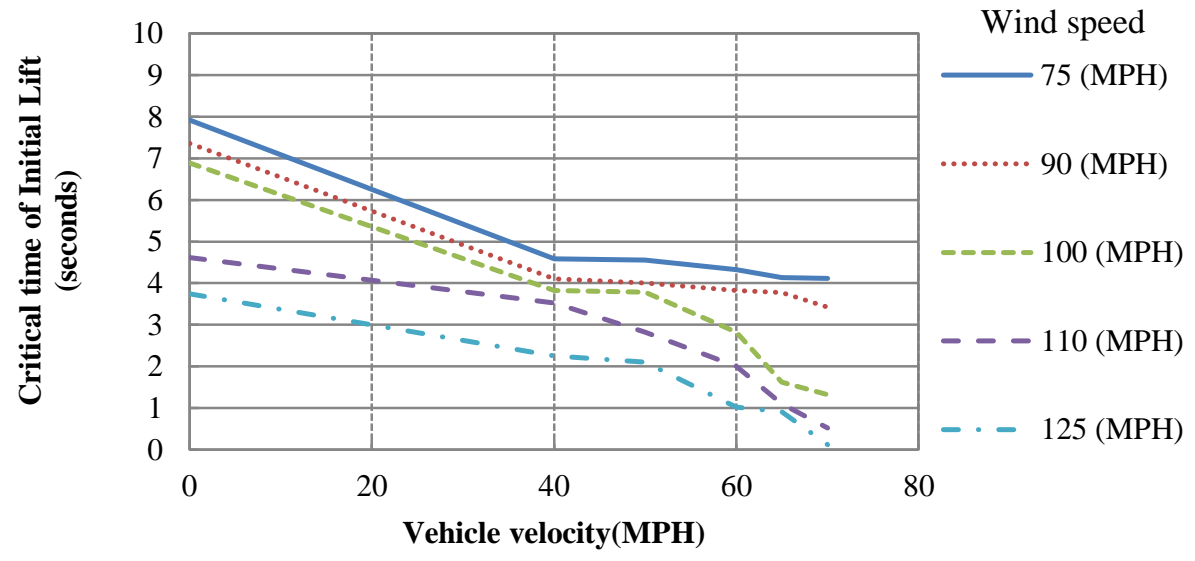

Figure 8.83: Critical sustained time for initial liftoff on dry pavement $(\mu=0.9)$ with full cargo load

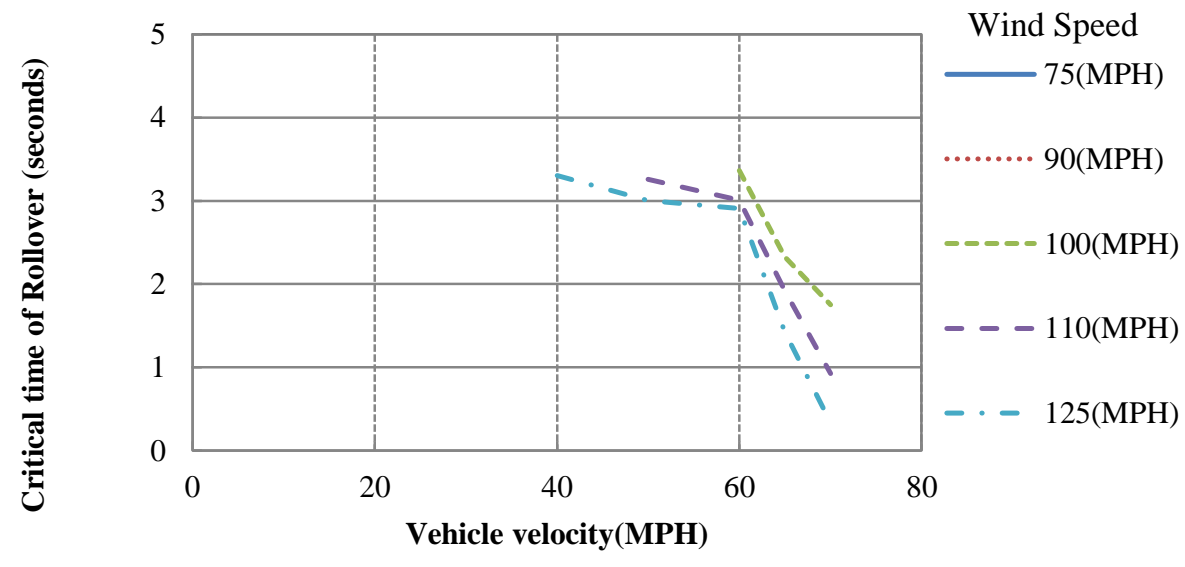

Figure 8.84: Critical sustained time for rollover on dry pavement $(\mu=0.9)$ with full cargo load

Figure 8.85 and Figure 8.86 show the results for a truck with $50 \%$ cargo load traveling at $40 \mathrm{MPH}$ on dry pavement. A steady wind of $75 \mathrm{MPH}$ lasting for $3.5 \mathrm{sec}$ would be sufficient for liftoff. Similar to the above results, no rollover would occur. 


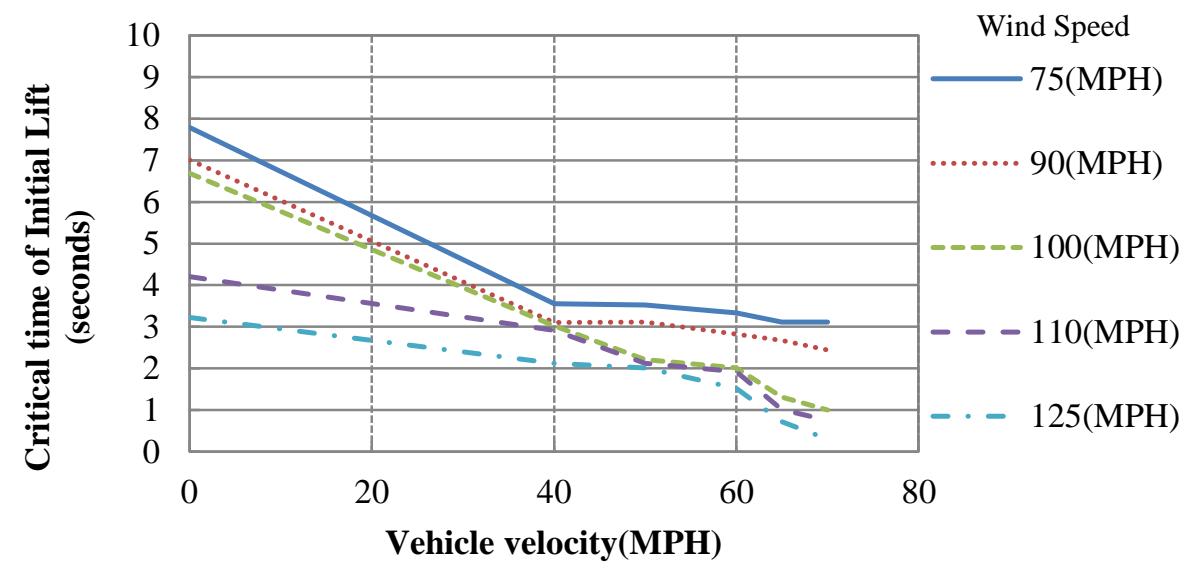

Figure 8.85: Critical sustained time for initial liftoff on dry pavement $(\mu=0.9)$ with $50 \%$ cargo load

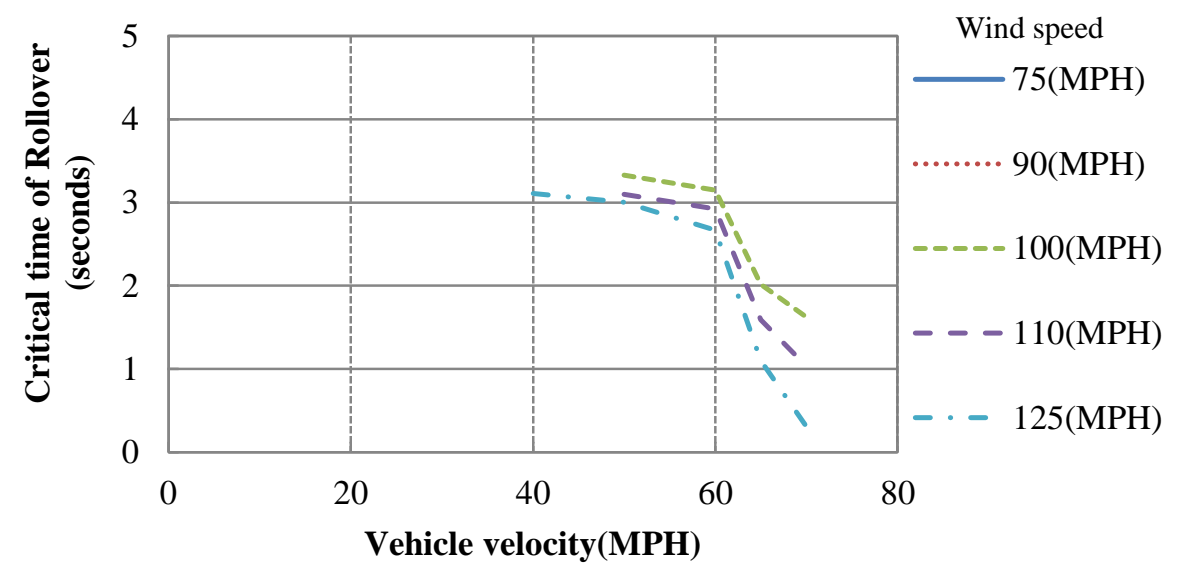

Figure 8.86: Critical sustained time for rollover on dry pavement $(\mu=0.9)$ with $50 \%$ cargo load

For the case with a $10 \%$ cargo load, Figure 8.87 and Figure 8.88 show that a vehicle traveling on dry pavement at $40 \mathrm{MPH}$ will have a wheel lift off at $3.2 \mathrm{sec}$, but rollover will not occur. Unlike the previous cases, rollover will occur, however, at a vehicle speed of $60 \mathrm{MPH}$. 


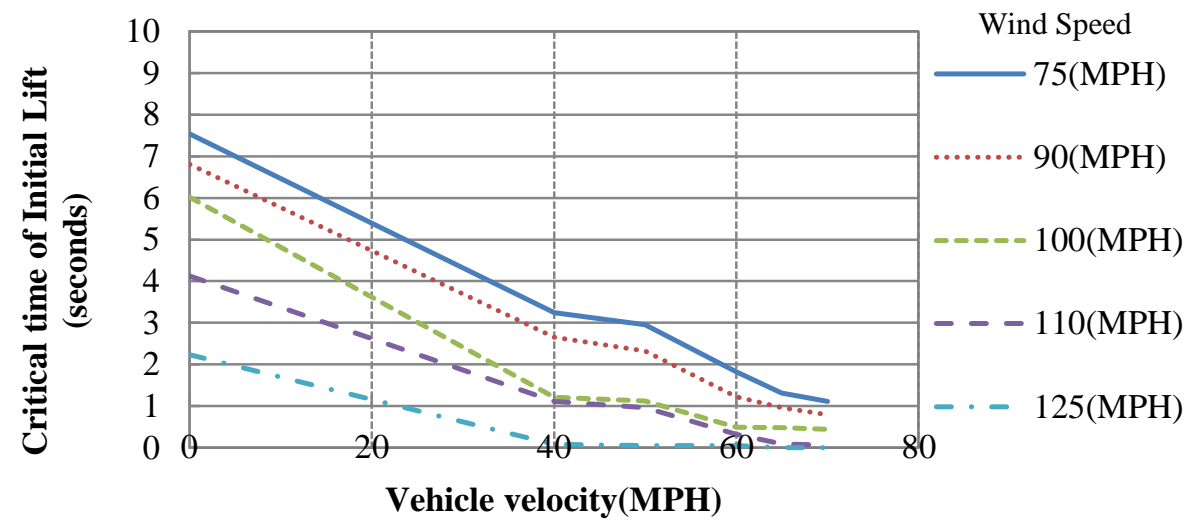

Figure 8.87: Critical sustained time for initial liftoff on dry pavement $(\mu=0.9)$ with $10 \%$ cargo load.

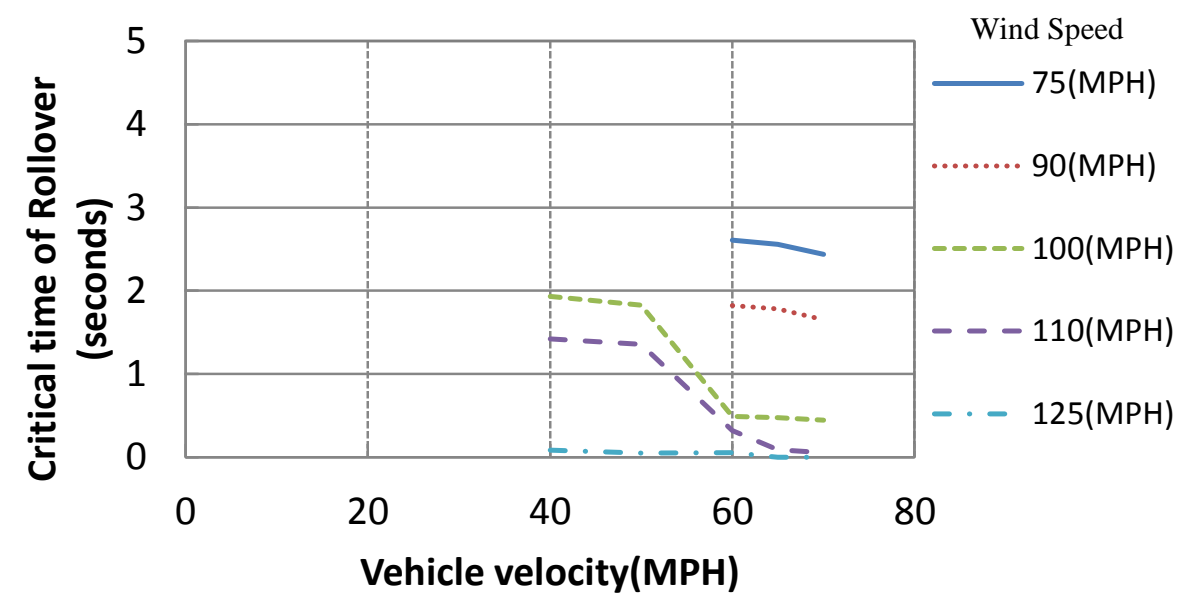

Figure 8.88: Critical sustained time for rollover on dry pavement $(\mu=0.9)$ with $10 \%$ cargo load.

Figure 8.89 to Figure 8.94 describe the response of a vehicle travelling on a wet road surface (COF $=0.5$ ) with full, partial and no cargo loading condition. For the full load case, Figure 8.89 and Figure 8.90 show the critical sustained times for initial liftoff and the onset of a rollover, respectively. The 0.5 value for the COF represented a wet surface. It is seen that at a vehicle speed of $40 \mathrm{MPH}$, it would take a $75 \mathrm{MPH}$ wind $4.6 \mathrm{sec}$ to cause a liftoff. However, at this vehicle speed and wind speed, no rollover would occur. This is similar to the case of a dry surface. 


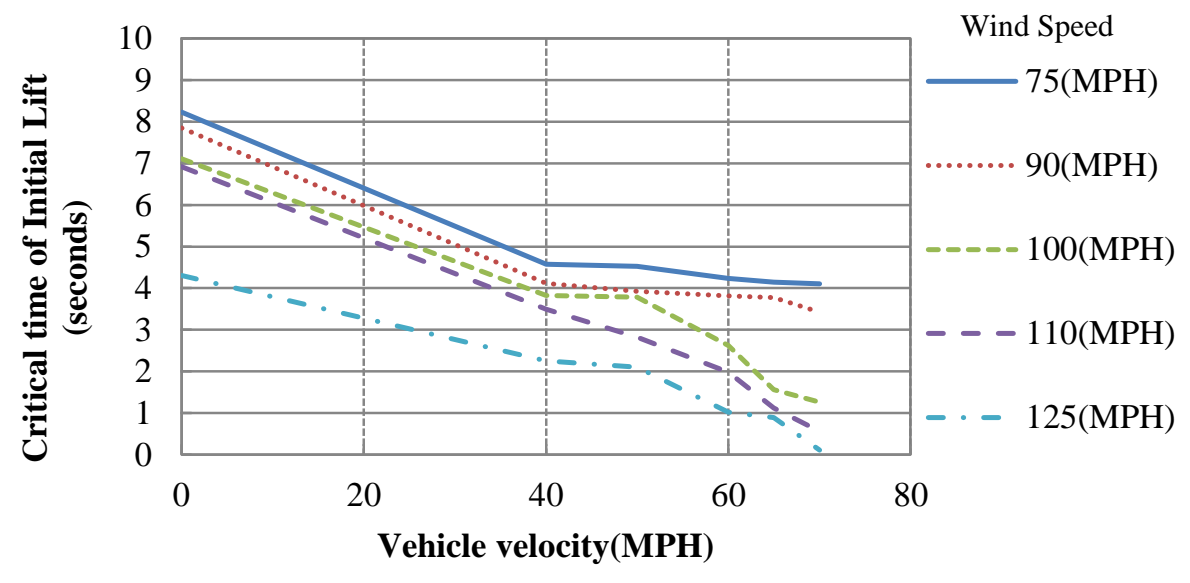

Figure 8.89: Critical sustained time for initial liftoff on wet pavement $(\mu=0.5)$ with full cargo load.

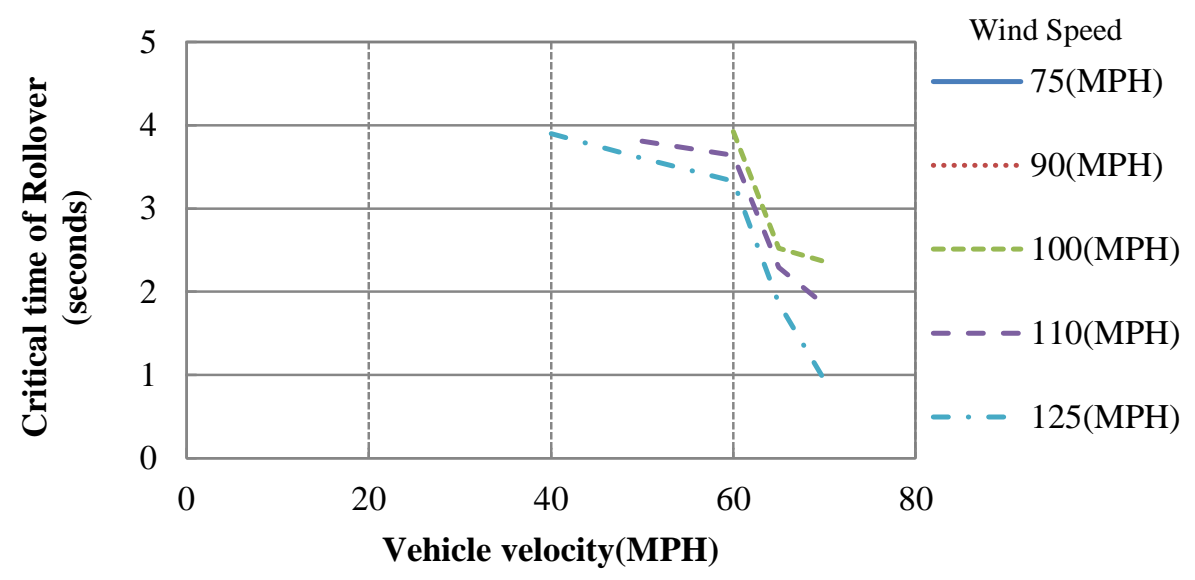

Figure 8.90: Critical sustained time for rollover on wet pavement $(\mu=0.5)$ with full load.

Figure 8.91 and Figure 8.92 show the results for a truck with $50 \%$ cargo loading traveling on wet pavement. At $40 \mathrm{MPH}$ a steady wind of $75 \mathrm{MPH}$ lasting for $3.5 \mathrm{sec}$ would be sufficient for liftoff. Similar to the above results, no rollover would occur. 


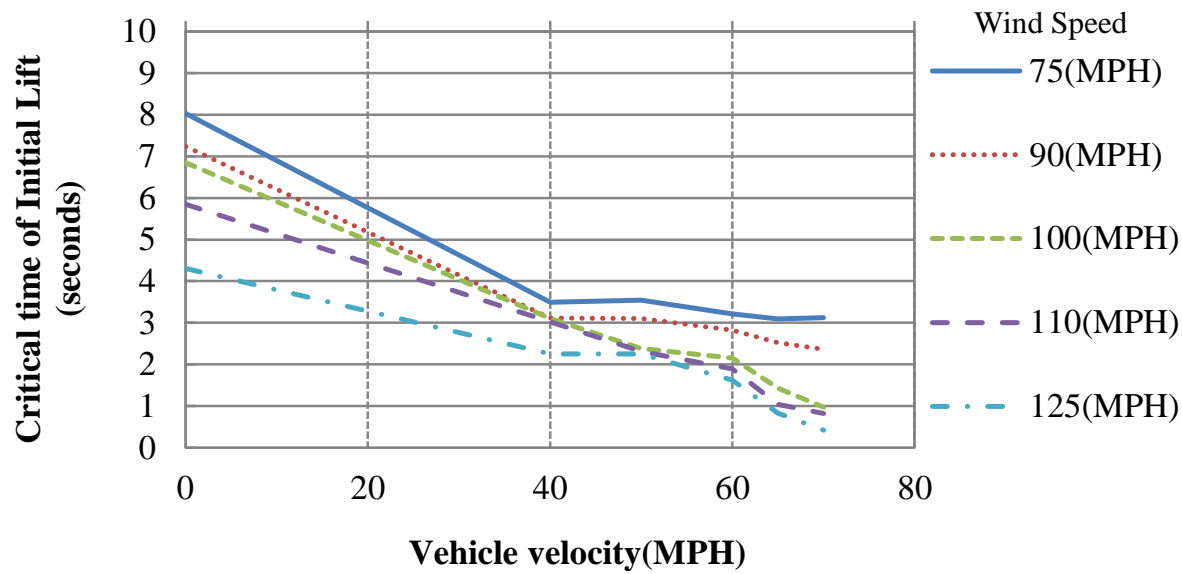

Figure 8.91: Critical sustained time for initial liftoff on wet pavement $(\mu=0.5)$ with $50 \%$ cargo load.

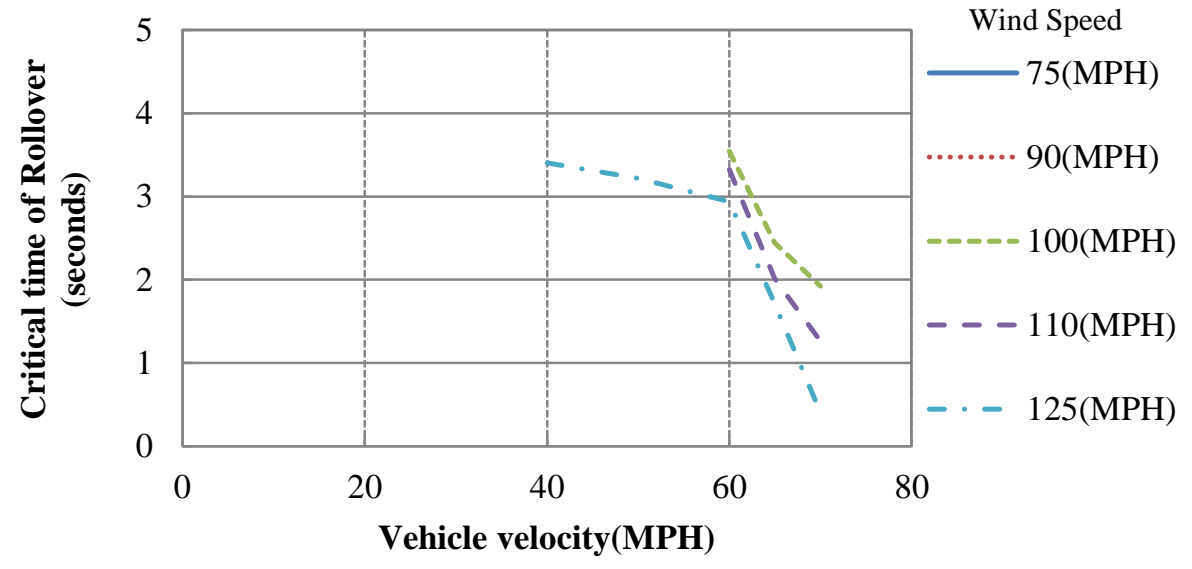

Figure 8.92: Critical sustained time for rollover on wet pavement $(\mu=0.5)$ with $50 \%$ cargo load.

For the case with a $10 \%$ cargo load, Figure 8.93 and Figure 8.94 show that a vehicle traveling on wet pavement at $40 \mathrm{MPH}$ will have a wheel lift off at $3.2 \mathrm{sec}$, but rollover will not occur. Unlike the previous cases, rollover will occur, however, at a vehicle speed of $60 \mathrm{MPH}$. 


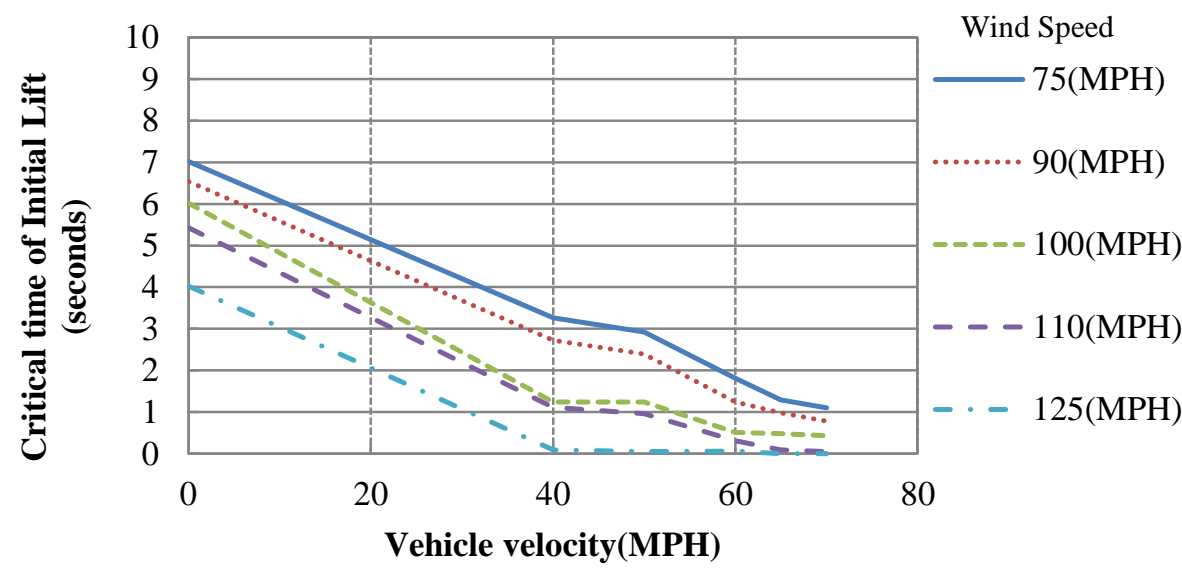

Figure 8.93: Critical sustained time for initial liftoff on wet pavement ( $\mu=0.5)$ with $10 \%$ cargo load).

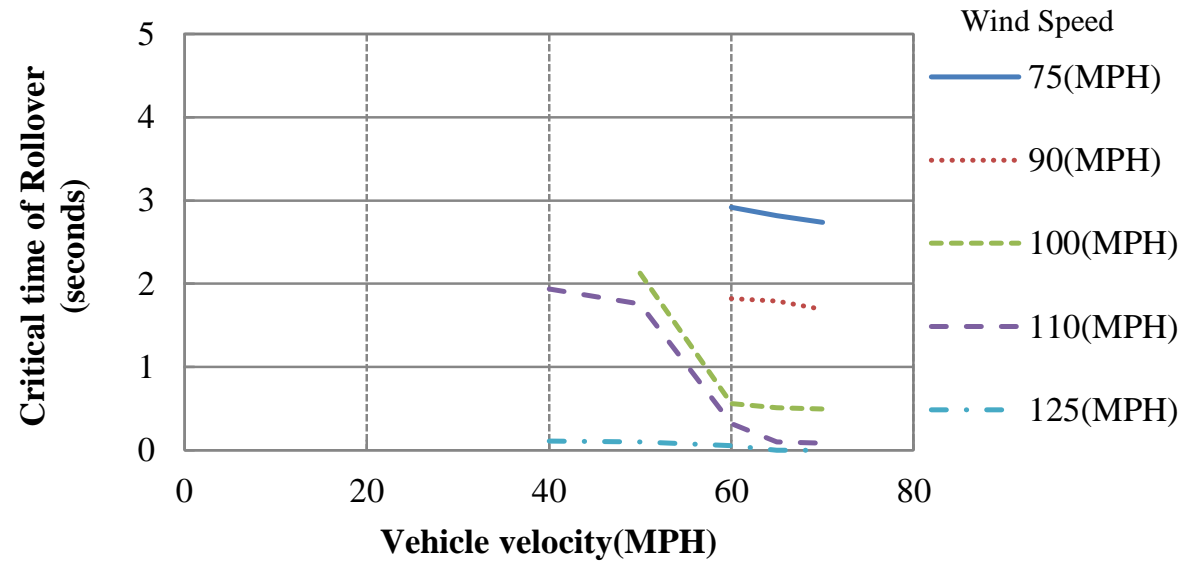

Figure 8.94: Critical sustained time for rollover on wet pavement $(\mu=0.5)$ with $10 \%$ cargo load.

As far as critical sustained times go, there does not seem to be any significant difference between the results for dry $(\mathrm{COF}=0.9)$ and wet pavement $(\mathrm{COF}=0.5)$.

\subsection{Sensitivity Analysis}

In addition to the deterministic LS-DYNA simulations described above, a sensitivity study using LS-OPT was performed for further identification of the correlation between the input parameters and the response of the vehicle. The sensitivity study was conducted for four variables: velocity of the wind (converted to the pressure exerted on the vehicle), velocity of the vehicle, friction coefficient between the tires and the ground and the mass of the cargo. The response variables were the lateral displacement of the center of gravity of the truck with cargo and the rotations about the longitudinal $(x)$ and vertical $(z)$ axes. The D-optimal point sampling method was used to choose 40 input vectors. Doptimal design is a method using an optimized subset of Factorial designs to minimize the approximation error. Bounds on the selected input parameters are listed in Table 8.10. 
Table 8.10: Parameter screening variables

\begin{tabular}{|l|l|l|l|}
\hline Parameter & \multicolumn{1}{|c|}{ Description } & \multicolumn{1}{c|}{ Lower bound } & \multicolumn{1}{c|}{ Upper Bound } \\
\hline rho & scaling factor for density of cargo & $0.1(0.28$ tonne $)$ & $1.0(2.8$ tonne $)$ \\
\hline fric & $\begin{array}{l}\text { friction coefficient between the ground } \\
\text { and tires }\end{array}$ & 0.1 & 0.9 \\
\hline velf & scaling factor for the prescribed velocity & $1(40 \mathrm{mph})$ & $1.75(70 \mathrm{mph})$ \\
\hline presf & $\begin{array}{l}\text { scaling factor for the lateral wind } \\
\text { pressure }\end{array}$ & $\begin{array}{l}1 \\
(206.61 \mathrm{~Pa}-40 \mathrm{mph})\end{array}$ & $\begin{array}{l}14.062 \\
(2905.4 \mathrm{~Pa}-150 \mathrm{mpa})\end{array}$ \\
\hline
\end{tabular}

The Radial Basis Function Network (RBFN) was chosen for building the Response Surface (RS). The sensitivity analysis was performed using the Sobol Indices technique (see LS-OPT manual for reference). Figure 8.95shows the results of this study. The most important variable for the rotation of the vehicle turned out to be the wind pressure. Variations in values of the pressure scaling factor were responsible for over $70 \%$ of the variations in the response of the vehicle. The friction coefficient was responsible for about $20 \%$ of them. The density of the cargo was responsible for about $6 \%$ of changes in the vehicle response.

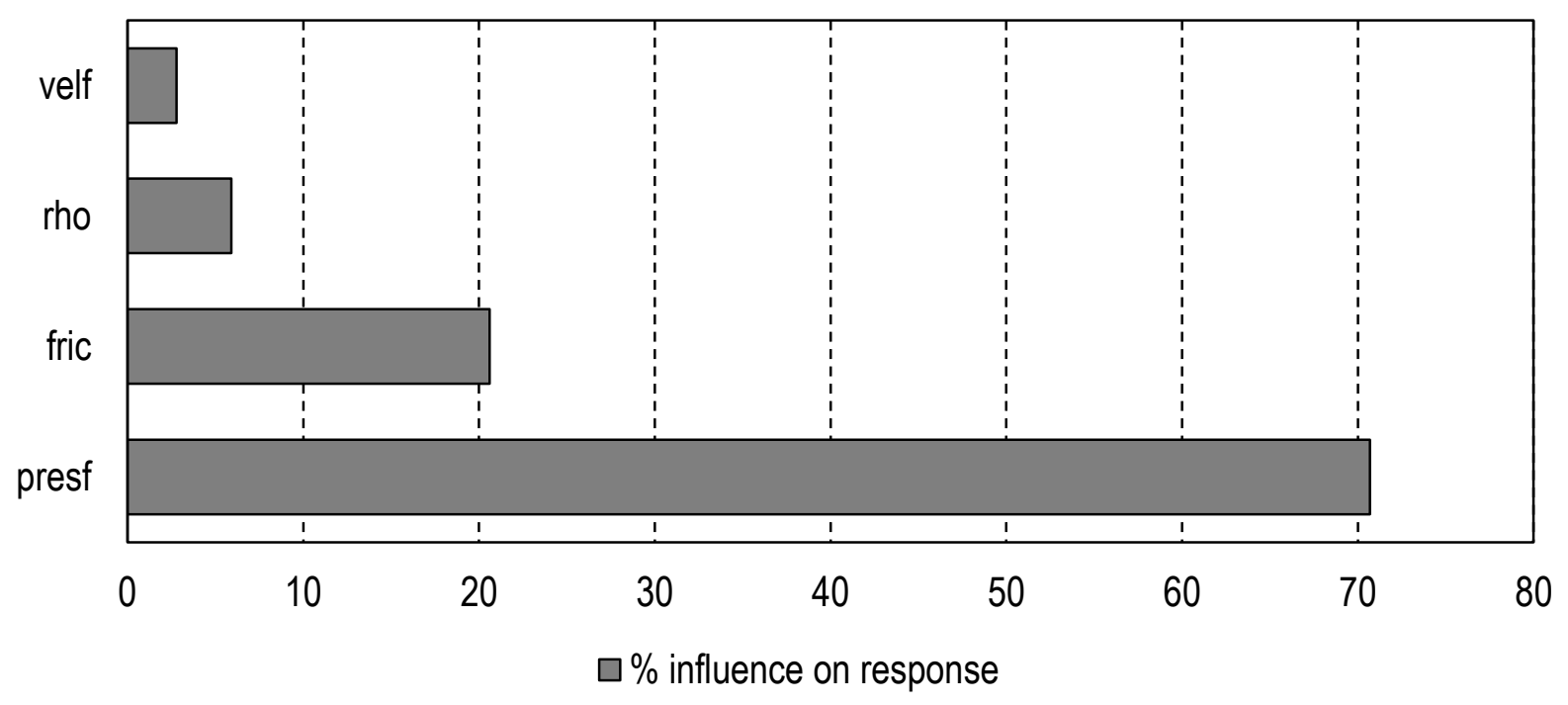

Figure 8.95: Sensitivity analysis results for the initial model

The response of the vehicle as a function of mentioned parameters is highly nonlinear and 40 simulation points used to create RBFN are not enough to provide enough accuracy for the results - the fitted surface is not capturing well the real response in the simulation points. For very nonlinear responses more simulations need to be performed to cover more precisely the design space and to build more accurate RS. The above results should be used for initial variable screening purposes only.

In the second set of runs velocity of the vehicle was eliminated from the list of input variables. Its influence on the response is minimal and keeping it in this sensitivity study only introduces large number 
of simulations. It was set as a constant at the level of $40 \mathrm{mph}$ as the drivers would naturally slow down at the adverse weather conditions. With the three parameters left (see Table 8.11) Full Factorial point sampling was used to select points for consecutive simulations. In factorial method the points are equidistant, what makes the space evenly covered with a grid of $5^{3}=125$ simulation points with 5 being the number of points in one dimension of that space. For the initial set of four parameters that number would have to be $5^{4}=625$ in order to keep the same level of accuracy.

Table 8.11: Parameter screening variables

\begin{tabular}{|l|l|l|l|}
\hline Parameter & \multicolumn{1}{|c|}{ Description } & Lower bound & Upper Bound \\
\hline rho & scaling factor for density of cargo & 0.1 & 1.0 \\
\hline fric & friction coefficient between the ground and tires & 0.1 & 0.9 \\
\hline presf & scaling factor for the lateral wind pressure & 1 & 14.062 \\
\hline
\end{tabular}

Tripled number of the simulation runs for lower number of variables increases significantly the accuracy of the results in comparison to the first analysis. Figure 8.96displays the results of sensitivity study analysis for it. The order of the significance of the variables did not change but their percentage contribution to the response did. The pressure scaling factor is responsible for about $61 \%$ of the changes in the response, the friction coefficient for about $23 \%$ and the remaining $17 \%$ can be attributable to the scaling factor for the density of the cargo.

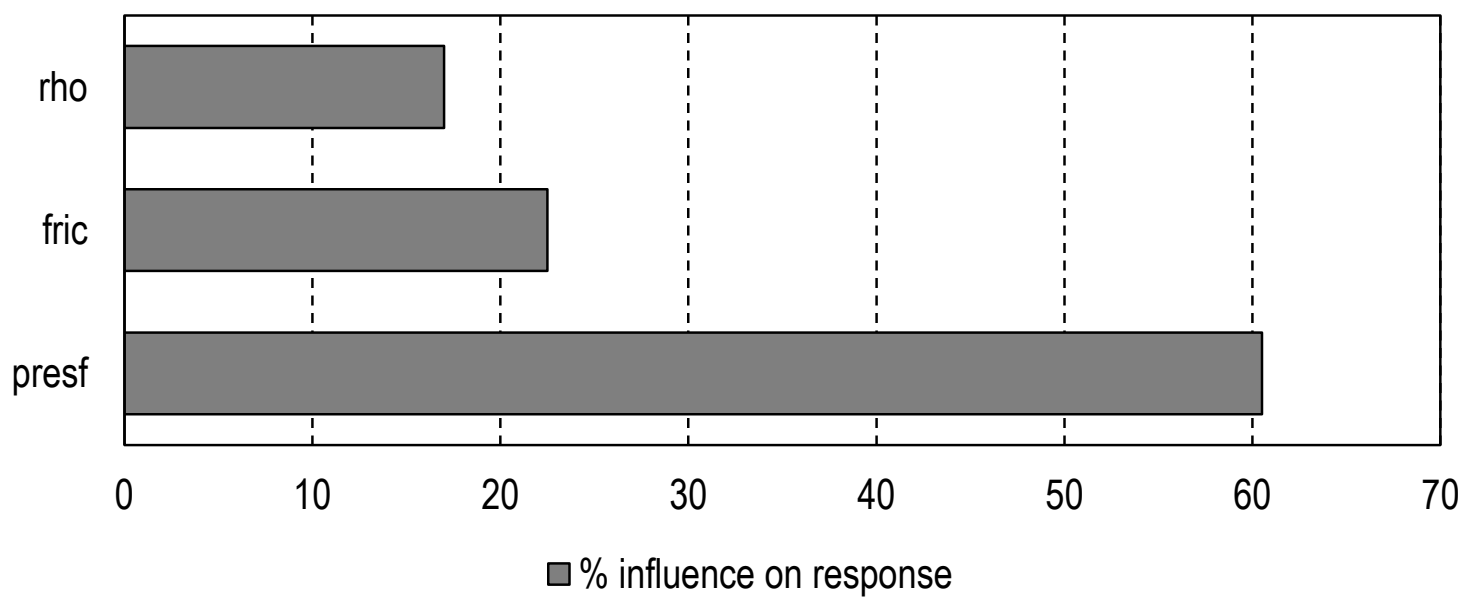

Figure 8.96: Sensitivity analysis results for the updated model 

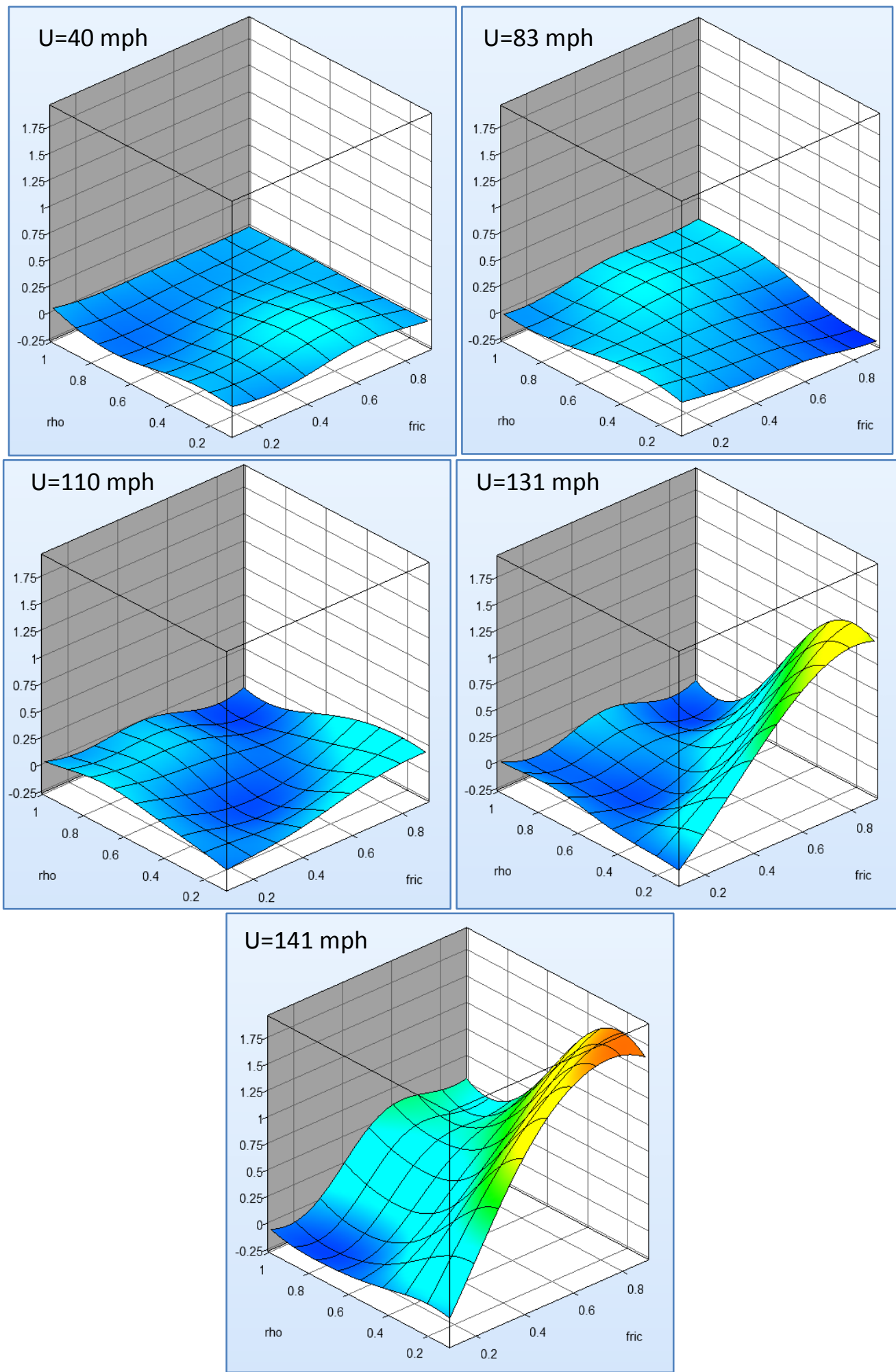

Figure 8.97: Approximate response surfaces for different values of pressure scaling factor 
Figure 8.97presents a set of plots for the fitted response surface as a function of friction coefficient and the scaling factor of the cargo's density. Six plots were prepared assuming different value of the third variable - the wind pressure scaling factor: $1,4,7,10,12,14$, which is equivalent to the wind speed of $40,82.6,109.8,131.4,141$ and $150 \mathrm{mph}$. It can be noted that the non-zero rotation is only present in the plots for higher velocities of the wind.

The Parallel coordinate plot is shown in Figure 8.98. It displays all the entities studied (i.e. input variables) and responses on separate parallel axes. Also it connects with a line the input values that were used in one simulation with the responses obtained in that simulation. Each path can be separately highlighted in the LS-OPT GUI. Following the axis with the rotation about the $x$ axis (response called $x \_r o t c c$ _resp) one can see that there were only two sets of values for that response - either close to zero, meaning no rotation of the vehicle or close to 1.6 radian, meaning 90 degree of rotation or full rollover. Also one can see that the paths to the $1.6 \mathrm{rad}$ for the rotation only lead through the highest values of the pressure scaling factor. Note that the response is defined as a final value of the time history. Thus, for zero value of the rotation ( $x_{-}$rot_cg_resp) the vehicle could lift up at the initial stage of the simulation but at the end of it all the wheels would be on the ground again. The defined response does not make any distinction between normal driving, side slip or lift up without rollover cases. For that different function would have to be defined as the response.

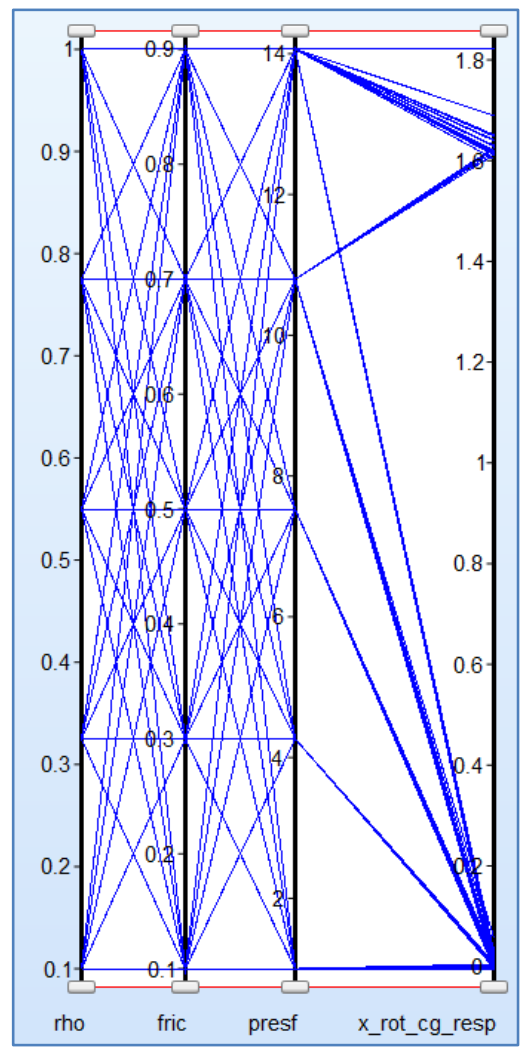

Figure 8.98: Parallel Coordinate plot 


\subsection{Reference:}

1. Ford Single Unit Truck, Reduced model (35,353 elements), 11/3/2008. [online] Available at: < http://www.ncac.gwu.edu/vml/models.html> [Accessed 01 June 2011].

2. F800 Single Unit Truck Model: User Manual, National Transportation Research Center, Inc., November 2005.

3. http://www.vehix.com/car-reviews/1999/ford/f-800/vehicle-specifications

4. Park, S. and Bosch, H., "Influence of Cross-Tie Geometry and Details on Mitigation of WindInduced Vibrations, "Wind Induced Vibrations of Cable Stayed Bridges Workshop, St. Louis, MO, April 25-27, 2006.

5. Ley, H. Transportation Research and Analysis Computing Center (TRACC) Year 5 Quarter 3 Progress Report, ANL-11/TRACC-USDOT-Y5Q2, August 2011.

\subsubsection{Electromagnetic Shock Absorber for Vehicle Stability under High Wind Conditions}

The work done during the fourth quarter involved FEM simulations of the Ford F800 truck model to obtain stiffness and damping properties of the suspension system. All of the properties of the F800 truck have been found and suitable gain values have been found for each location. The evaluation of the EMSA quarter-car control model in Matlab-Simulink for each location of the truck has been completed. The results indicate an improvement for reduction of the suspended mass acceleration, while maintaining road holding ability and satisfying other requirements such as deflection limit of the suspension.

\subsection{Simulation of theF800 Truck Model}

The simulation to obtain the effective stiffness of the leaf springs in the suspension of the F800 truck was performed in a similar manner than the simulation to obtain the tire stiffness values. Almost every component/part was removed from the F800 model except for the wheel/tire assembly, the suspension assembly and a nearly rigid beam that is attached directly to the suspension as shown in Figure 8.99. 


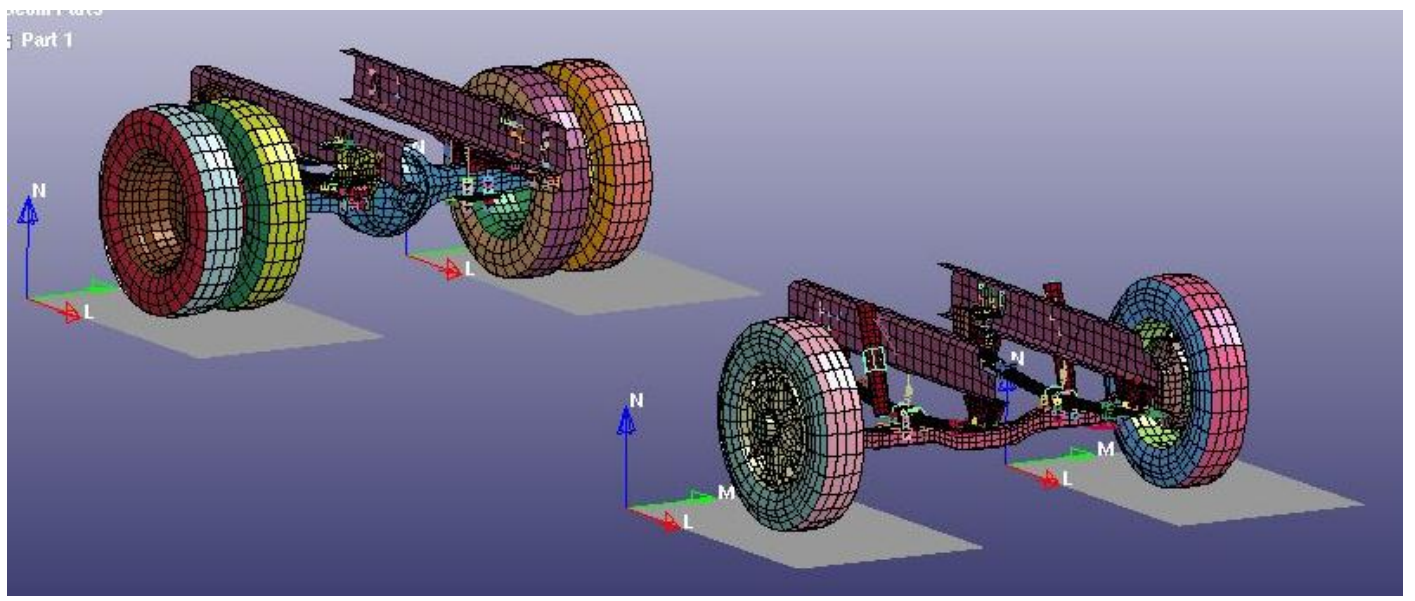

Figure 8.99: Screenshot of the suspension simulation

The nearly rigid beams density was increased to mimic the actual load of the full F800 truck model. After allowing the F800 to initialize, the movement of the wheel/tire assembly in Z-direction (vertical) was constrained to not move. A vertical displacement (prescribed motion) to the beam was then applied downward and the resulting reaction force from the wheel/tire assembly was recorded. Figure 8.100 is a plot of the reaction force from the wheel/tire assembly versus the deformation of the front passenger suspension. Notice in the very beginning the model is initializing.

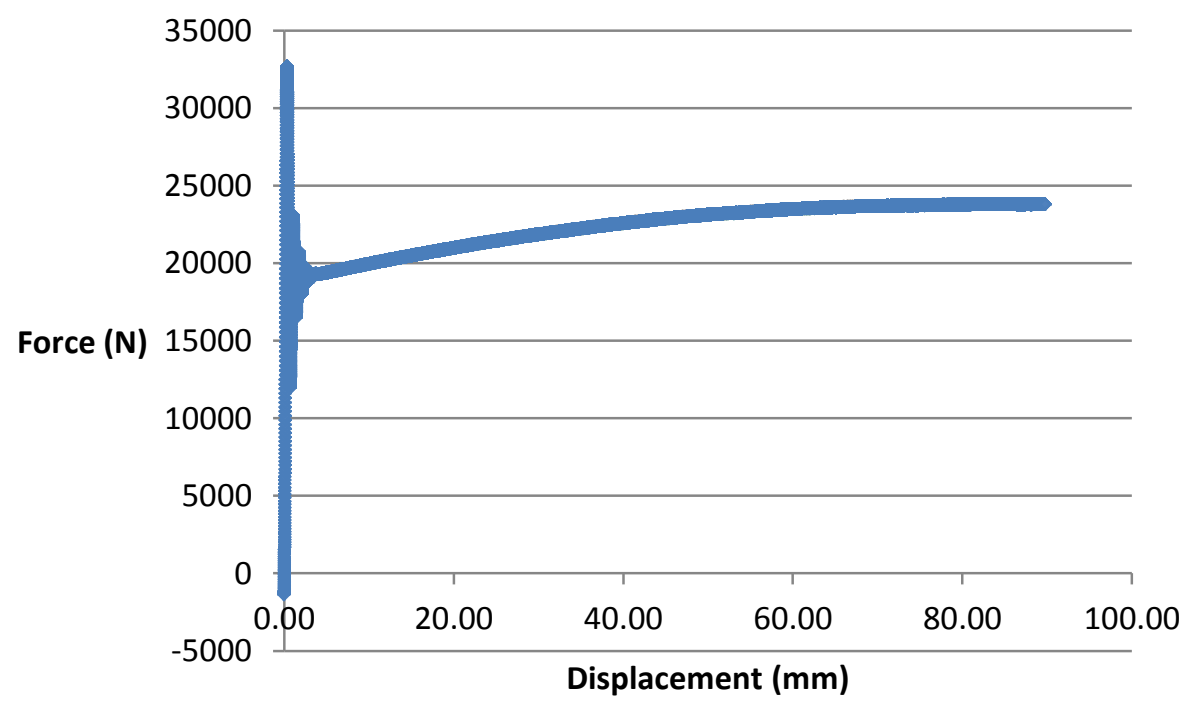

Figure 8.100: Force response vs. displacement of beam for front passenger location

Knowing that the maximum deformation for the suspension is around $40 \mathrm{~mm}$, Figure 8.101 shows the range of interest and a linear regression line. Note that the slope of line in Figure 8.101 is the effective 
stiffness of the suspension for the given location. The results for the front driver location are omitted for brevity given that they are nearly identical to what is shown in Figure 8.100 and Figure 8.101.

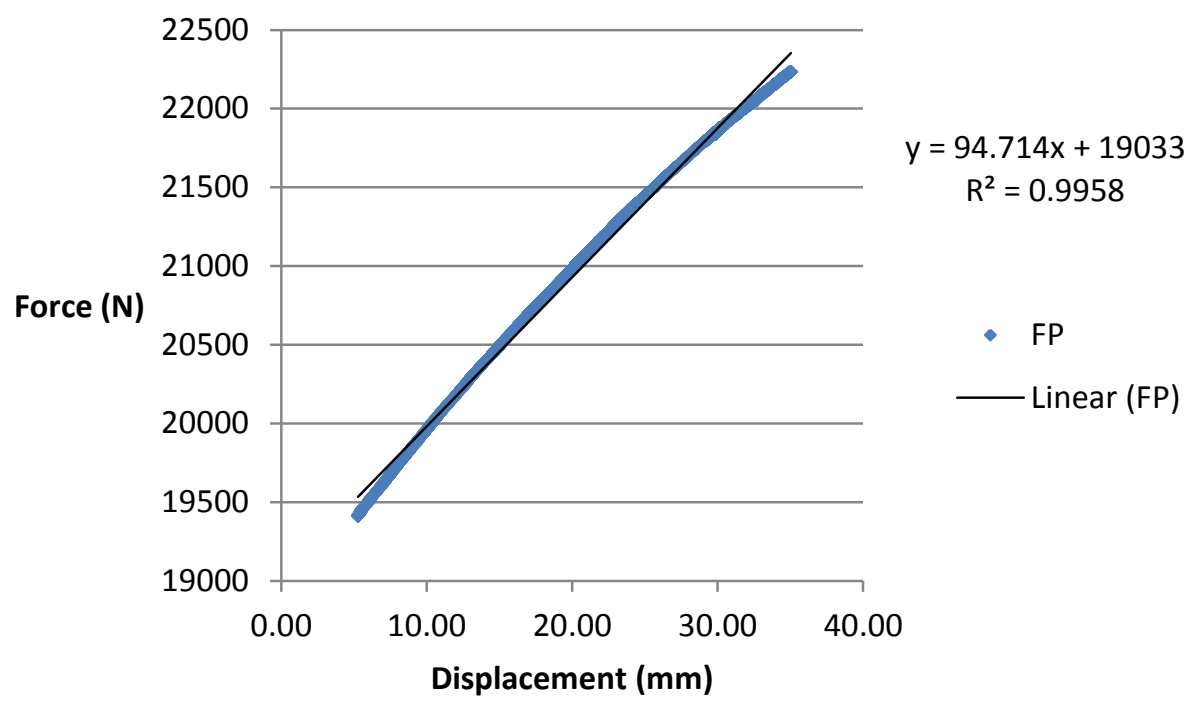

Figure 8.101: Force response vs. displacement for the range of interest

Figure 8.102 is a plot of the reaction force from the wheel/tire assembly verse the deformation of the rear driver suspension. Knowing that the maximum deformation for the suspension is around $40 \mathrm{~mm}$, Figure 8.103 shows the range of interest and a linear regression line. Note that the slope of the line in Figure 8.103 is the effective stiffness of the suspension for the given location. The results for the rear passenger location are omitted for brevity given that they are nearly identical to what is shown in Figure 8.102 and Figure 8.103. 


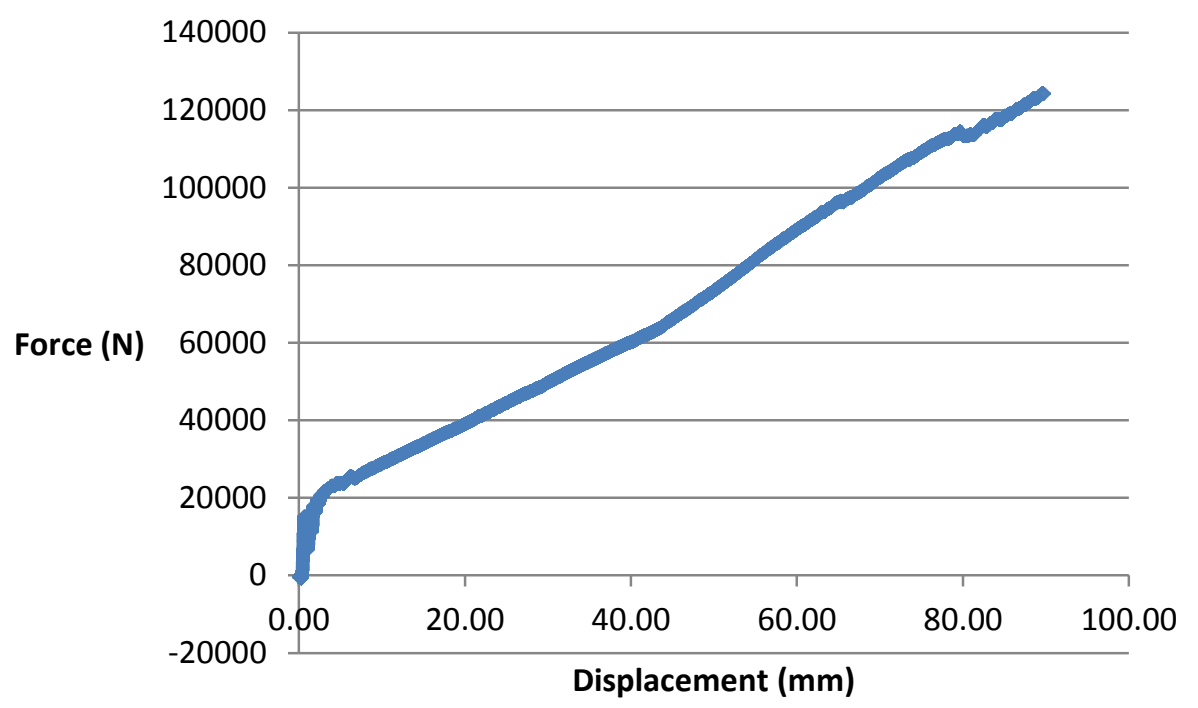

Figure 8.102: Force response vs. displacement of beam for rear driver location

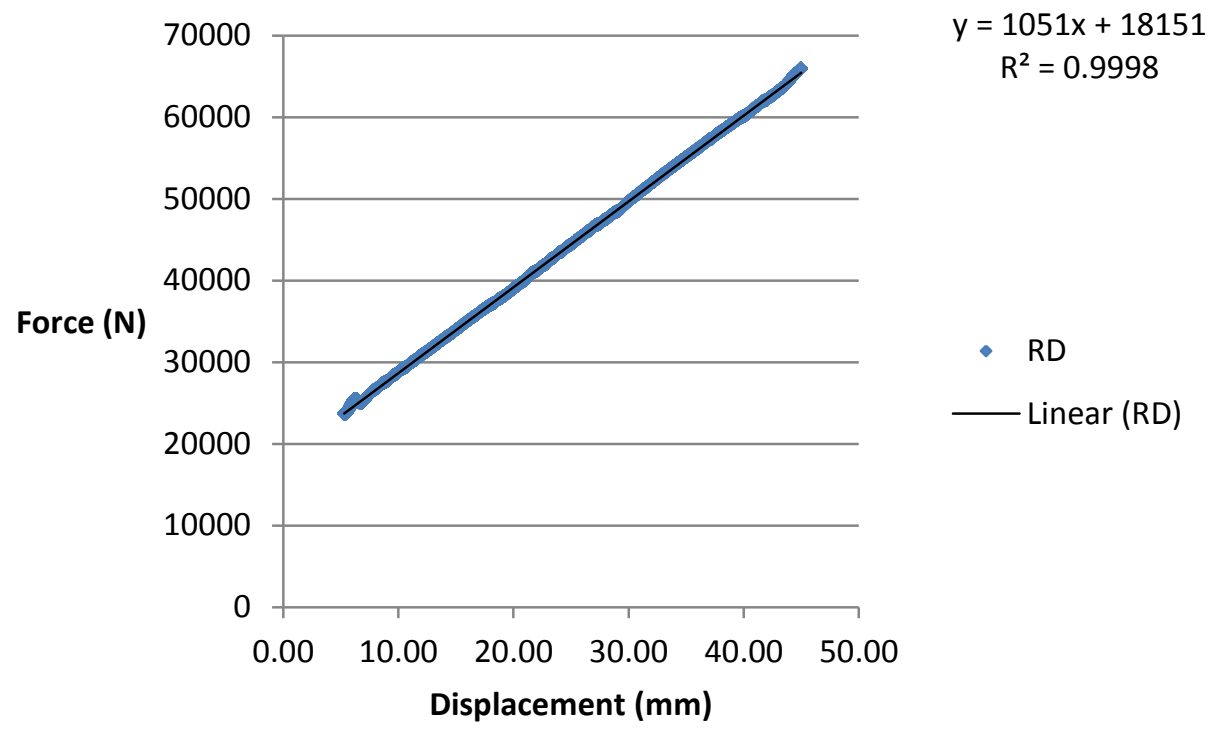

Figure 8.103: Force response vs. displacement for the range of interest

The stiffness values of the suspension for each location are shown in Table 8.12:. The percent difference for the front stiffness values is $0.19 \%$ and the percent difference for the rear stiffness values is $3.39 \%$. 
Table 8.12: Suspension stiffness values for each location

\begin{tabular}{|l|l|l|}
\hline$K_{R P}$ & 1087300 & $\mathrm{~N} / \mathrm{m}$ \\
\hline$K_{R D}$ & 1051000 & $\mathrm{~N} / \mathrm{m}$ \\
\hline$K_{F D}$ & 94893 & $\mathrm{~N} / \mathrm{m}$ \\
\hline$K_{F P}$ & 94714 & $\mathrm{~N} / \mathrm{m}$ \\
\hline
\end{tabular}

In order to obtain the damping coefficients for the suspension a vertical displacement (prescribed motion) was applied to the beam and then the beam was released, effectively allowing the system to vibrate freely. Figure 8.104 shows the free damped response of the beam for the front driver side.

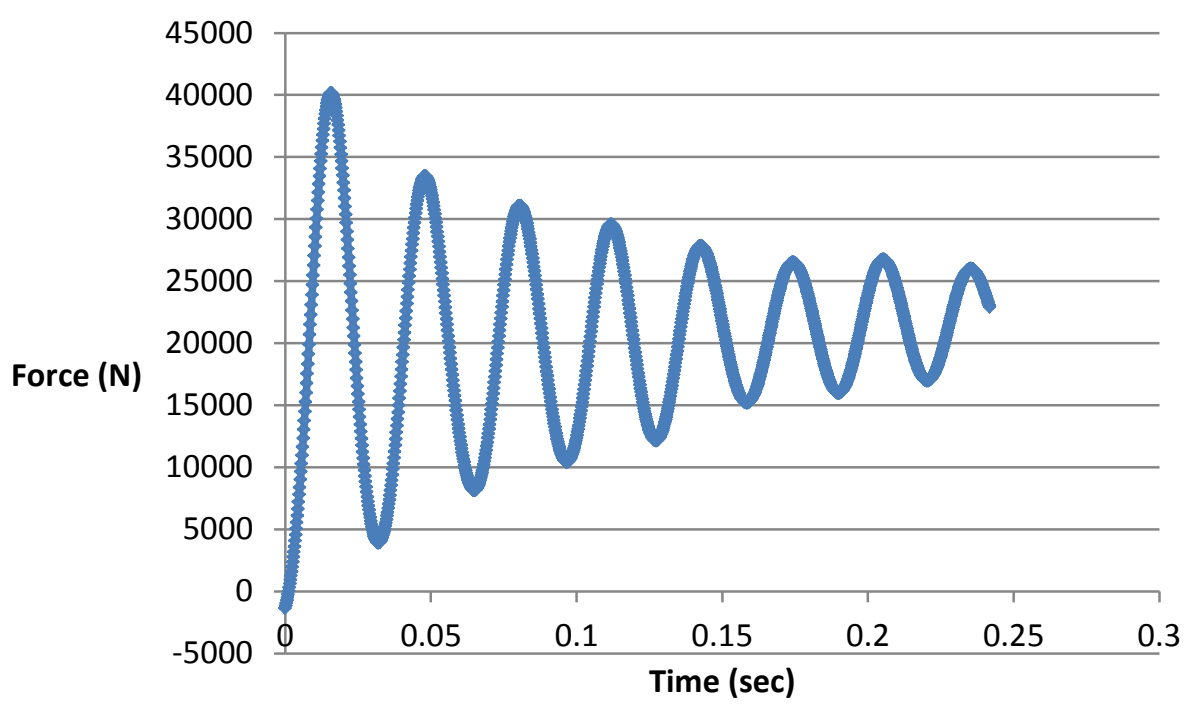

Figure 8.104: Front response vs. time for front driver location

The other locations had similar results and are omitted for brevity. The log-decrement method was applied to Figure 8.104 and after some calculations the damping coefficients were then determined. The damping values for the various locations of the suspension are shown in Table 8.13:.

Table 8.13: Damping coefficients of the suspension for each location

\begin{tabular}{|c|c|c|c|}
\hline $\begin{array}{c}\text { Rear Driver } \\
{[\mathrm{Ns} / \mathrm{m}]}\end{array}$ & $\begin{array}{c}\text { Rear } \\
\text { Passenger } \\
{[\mathrm{Ns} / \mathrm{m}]}\end{array}$ & $\begin{array}{c}\text { Front Driver } \\
{[\mathrm{Ns} / \mathrm{m}]}\end{array}$ & $\begin{array}{c}\text { Front } \\
\text { Passenger } \\
{[\mathrm{Ns} / \mathrm{m}]}\end{array}$ \\
\hline 447.702 & 321.130 & 6407.560 & 6918.625 \\
\hline
\end{tabular}




\subsection{Controller Formulation}

In order to find suitable gain values for the controller, the performance index needs to be defined. The performance index is user defined criteria based upon dynamics of the model and the states of interest [1]. The performance index is typically given in the form of an integral of a cost function, which is to be minimized. Shown in equation (8.14) is the general form of the performance index, where $L(X(t), u(t))$ is the cost function to be minimized. $X(t)$ is the state vector at time $t$ and $u(t)$ is the desired force (see equation (8.14) in [2]).

$$
I=\int_{0}^{t_{f}} L(X(t), u(t)) d t
$$

The cost function is shown in equation (8.15), where $Q$ is a $n \times n$ constant coefficient diagonal matrix and $R$ is a $m \times m$ constant coefficient matrix. The value of $n$ is equal to the number of states and $m$ is equal to the number of control inputs, so $n$ is 4 and $m$ is 1 . Note, $L(X(t)$ and $u(t))$ will be scalar values.

$$
L\left(X(t), u_{d}(t)\right)=X^{T}(t) * Q * X(t)+u^{T}(t) * R * u(t)
$$

The product of $X^{T}(t) * Q * X(t)$ represents the cost associated with the states or the amount of time it takes to have them decay, the evaluation of this product is shown in equation (8.16).

$$
X^{T}(t) * Q * X(t)=q_{1} * X_{1}^{2}+q_{2} * X_{2}^{2}+q_{3} * X_{3}^{2}+q_{4} * X_{4}^{2}
$$

The product of $u^{T}(t) * R * u(t)$ represents the cost associated with control or the amount of cost that will appropriately decay the response, the evaluation of this product is shown in equation (8.17).

$$
u^{T}(t) * R * u(t)=r_{1} * u^{2}
$$

Note that $Q$ and $R$ are determined by the designer preference and that the larger the cost or weight associated with $Q$ will in turn make the cost or weight associated with $R$ smaller. When $Q$ and $R$ are determined, the gain values are found by using Matlab's built-in function "Iqr". Shown in equation (8.18) is the Matlab command, where $A$ and $B$ are the matrices shown in equations (8.11) and (8.12) in [2]. 


$$
K=\operatorname{lqr}[A, B, Q, R]
$$

\subsection{Matlab-Simulink Results}

The current Matlab-Simulink model incorporates the EMSA model with the quarter-car control model as well as the input road profile. It includes a Matlab script or code that allows the user to input parameters/properties of the desired quarter-car model as well as modifying values that are used to formulate the matrix, $K$. After running the code, the Simulink model can then evaluate the dynamics of the quarter-car model given the properties of the system and the controller design. At this point, the data is then passed to Microsoft Excel for post-processing. The Excel file automatically calculates the

RMS (Root-mean-square) values for the states, $\ddot{x}_{s}$ and $\ddot{x}_{u}$ (The accelerations of the sprung and unsprung masses). The Excel file also creates two plots that critique the performance of the controller in terms of maximum suspension deformation and road holding ability. Multiple trials were performed using properties and gain values obtained from [3] and [4]. These trials were performed to ensure the correctness of the model.

Four more sets of trials were performed in order to obtain suitable gain values for each location of the vehicle. After the gain values were determined, the results were analyzed to ensure that the performance of the vehicle suspension system was improved in terms of comfort and road holding ability as well as satisfying the physical constraints. Shown in Figure 8.105 is the entire Simulink model, the block diagram of this model is shown in Figure 8.29 in [2]. It is very hard to understand the model by simply examining Figure 8.105 due to the details in the image. 


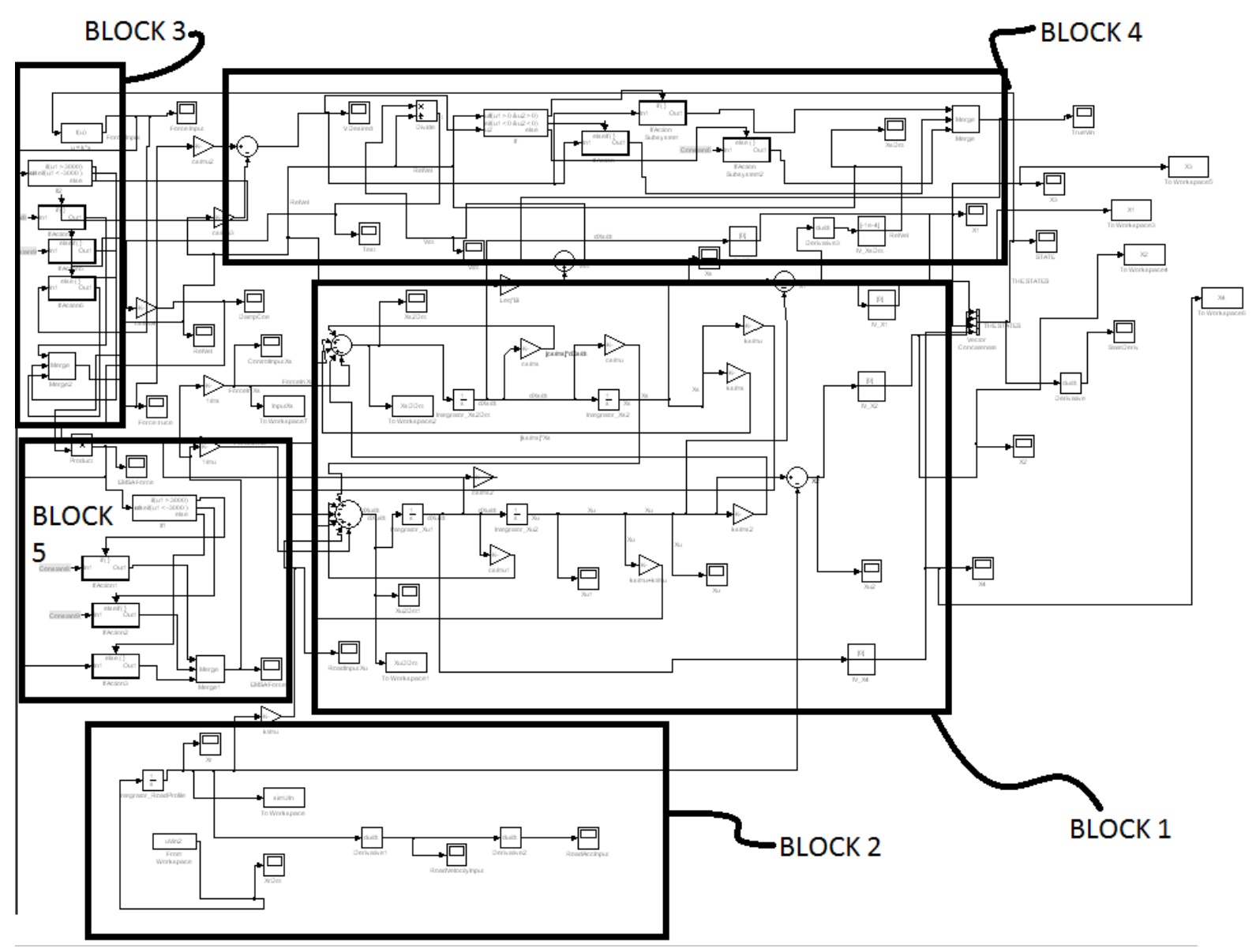

Figure 8.105: Screenshot of the entire Simulink model

In order to explain the model effectively, Figure 8.105 was separated into "Blocks" as shown. Block 1 is the quarter-car dynamics; this involves two 2 nd order ordinary differential equations. In other words block 1 is basically equations (8.8) and (8.9) in [2]. Block 2 involves the road profile that represents the road disturbance. Block 3 is the controller portion; this includes equation (8.14) in [2] and also has conditional statements that limit the desired force value due to the saturation or limit of the EMSA. Blocks 4 and 5 are the reverse model of the EMSA, which is based upon the desired force. They output the actual EMSA force that is then sent to Block 1. Blocks 4 and 5 also include two conditional statements. One conditional statement ensures proper operation of the EMSA by checking if the generated voltage is acting in the opposite direction then the desired voltage. The other conditional statement ensures that the EMSA force is limited to the absolute maximum producible force of the EMSA.

Since this model is designed for a quarter-car system, the front driver and passenger as well as the rear driver and passenger locations were analyzed using the Matlab-Simulink model and the results are compared with the passive systems. The most important aspects to consider are: the acceleration of the vehicle body, $\ddot{x}_{s}$, the tire deflection, $X_{2}$, the EMSA force, and the suspension deflection, $X_{1}$. 
Shown in Table 8.14: are the gain values used for the front driver semi-active simulation. The road input for this simulation is shown in Figure 8.14 in [5] and it is assumed that the vehicle is traveling at a constant velocity of $30 \mathrm{~m} / \mathrm{s}$. For each simulation the road input remains constant as well as the vehicles velocity.

Table 8.14: Gain values for the front driver location

\begin{tabular}{|c|c|c|c|}
\hline$K_{1}$ & $K_{2}$ & $K_{3}$ & $K_{4}$ \\
\hline-1.717 & 18980.772 & -96.404 & 441.724 \\
\hline
\end{tabular}

Table 8.15: displays the RMS values of the passive and semi-active cases, note that a negative percent improvement indicates the opposite of improvement. Although the semi-active RMS value for the suspension deflection, $X_{1}$, is slightly larger than that of the passive value as shown in Table 8.15:, the suspension deflection in both cases are much lower than the deflection limit which is $0.004 \mathrm{~m}$.

Table 8.15: RMS value comparison for the front driver location

\begin{tabular}{|c|c|c|c|}
\hline & Passive & Semi-Active & $\begin{array}{c}\text { Percent } \\
\text { Improvement }\end{array}$ \\
\hline$\ddot{X}_{s}$ & $0.2182\left(\frac{m}{s^{2}}\right)$ & $0.1347\left(\frac{m}{s^{2}}\right)$ & 38.28 \\
\hline$\ddot{X}_{u}$ & $7.5412\left(\frac{m}{s^{2}}\right)$ & $0.6718\left(\frac{m}{s^{2}}\right)$ & 91.10 \\
\hline$X_{1}$ & $0.001862(m)$ & $0.001907(m)$ & -2.40 \\
\hline$X_{2}$ & $0.000253(m)$ & $6.219 \mathrm{E}-05(m)$ & 75.40 \\
\hline$X_{3}$ & $0.01867\left(\frac{m}{s}\right)$ & $0.01884\left(\frac{m}{s}\right)$ & -0.91 \\
\hline$X_{4}$ & $0.04274\left(\frac{m}{s}\right)$ & $0.01602\left(\frac{m}{s}\right)$ & 62.51 \\
\hline
\end{tabular}

Figure 8.106 displays the plot of acceleration of the vehicle body for both passive and semi-active cases. The tire deflection plot for both cases is shown in Figure 8.107. Figure 8.108 is a plot of the suspension deflection for both cases. The EMSA force plot is shown in Figure 8.109. 


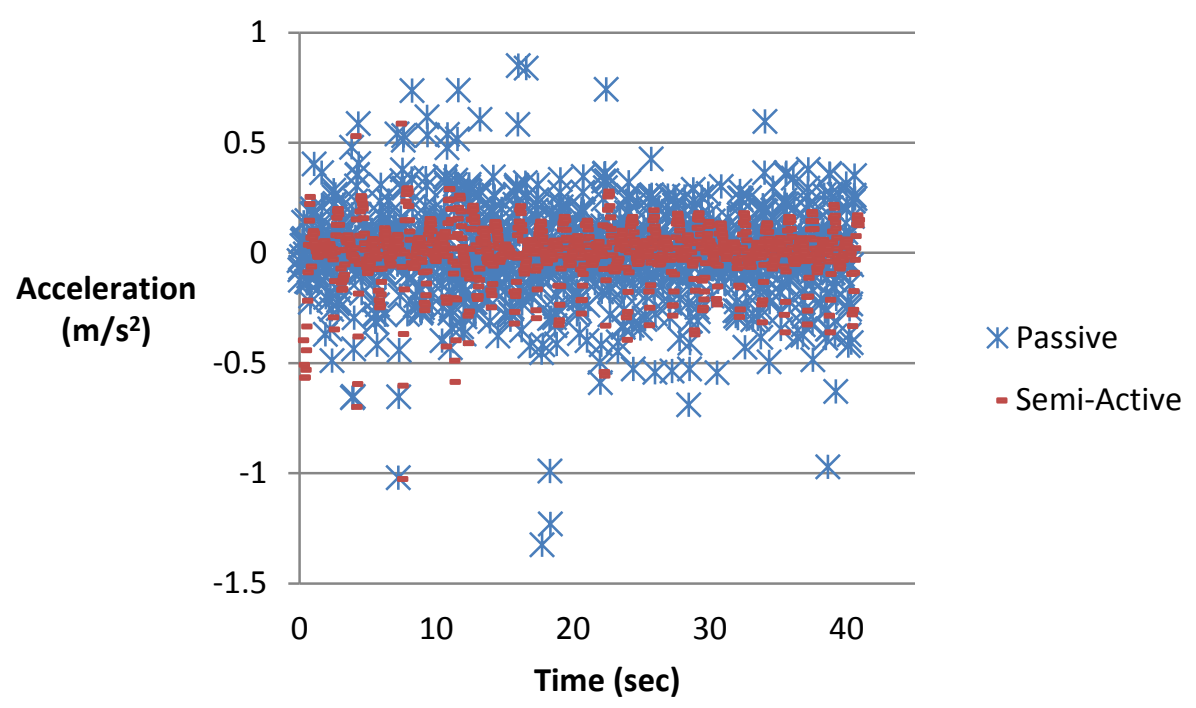

Figure 8.106: Front driver vehicle body acceleration

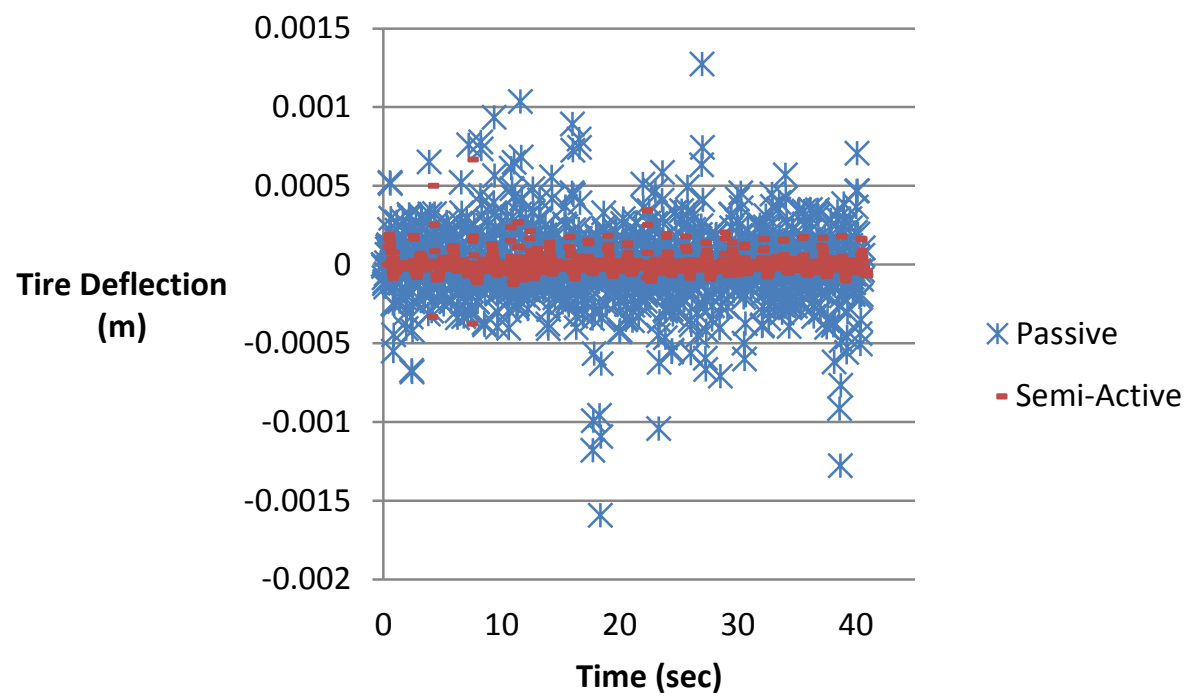

Figure 8.107: Front driver tire deflection 


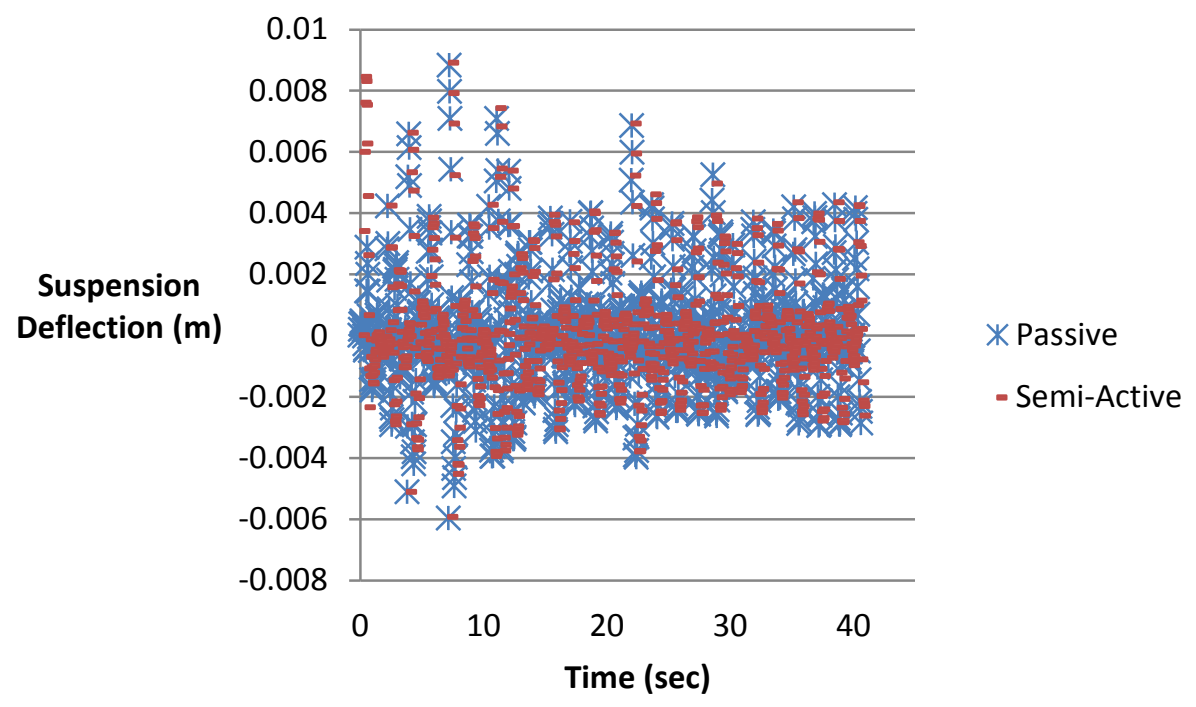

Figure 8.108: Front driver suspension deflection

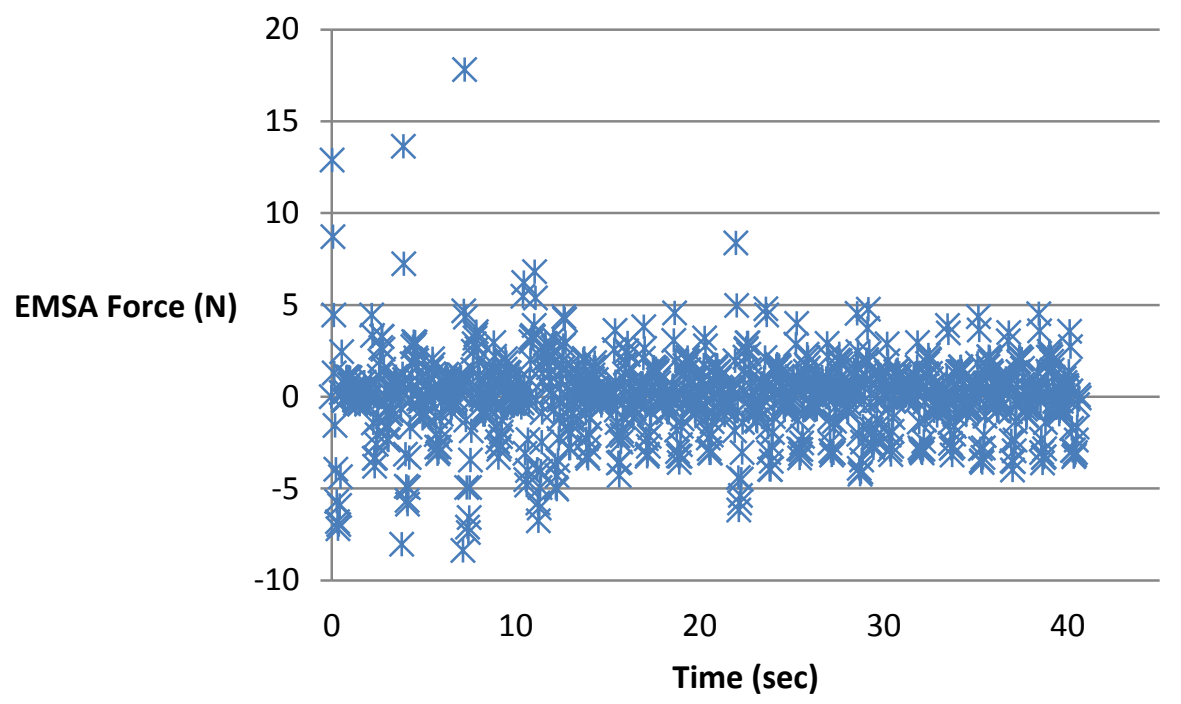

Figure 8.109: Front driver EMSA force input

Shown in Table 8.16: are the gain values used for the front passenger semi-active simulation. Notice that these gains are very close in magnitude to the gains shown in Table 8.14:. The plots for the front passenger location of the acceleration of the vehicle body, $\ddot{x}_{s}$, the tire deflection, $X_{2}$, the EMSA force, and the suspension deflection, $X_{1}$ are omitted for brevity since they nearly match Figures: Figure 8.106, Figure 8.107, Figure 8.108, and Figure 8.109. Table 8.17: displays the RMS values of the passive and semi-active cases. 
Table 8.16: Gain values for the front passenger location

\begin{tabular}{|c|c|c|c|}
\hline$K_{1}$ & $K_{2}$ & $K_{3}$ & $K_{4}$ \\
\hline-1.720 & 18963.922 & -90.200 & 411.007 \\
\hline
\end{tabular}

Table 8.17: RMS values for the front passenger location

\begin{tabular}{|c|c|c|c|}
\hline & Passive & Semi-Active & $\begin{array}{c}\text { Percent } \\
\text { Improvement }\end{array}$ \\
\hline$\ddot{X}_{s}$ & $0.2275\left(\frac{m}{s^{2}}\right)$ & $0.1255\left(\frac{m}{s^{2}}\right)$ & 44.84 \\
\hline$\ddot{X}_{u}$ & $6.3040\left(\frac{m}{s^{2}}\right)$ & $0.5963\left(\frac{m}{s^{2}}\right)$ & 90.54 \\
\hline$X_{1}$ & $0.001783(m)$ & $0.001748(m)$ & 1.96 \\
\hline$X_{2}$ & $0.000227(m)$ & $5.91 \mathrm{E}-05(m)$ & 73.93 \\
\hline$X_{3}$ & $0.01825\left(\frac{m}{s}\right)$ & $0.01797\left(\frac{m}{s}\right)$ & 1.50 \\
\hline$X_{4}$ & $0.04412\left(\frac{m}{s}\right)$ & $0.01620\left(\frac{m}{s}\right)$ & 63.28 \\
\hline
\end{tabular}

Shown in Table 8.18: are the gain values used for the rear driver semi-active simulation. Notice that these values are much different from those shown in Table 8.14: and Table 8.16:.

Table 8.18: Gain values for the rear driver location

\begin{tabular}{|c|c|c|c|}
\hline$K_{1}$ & $K_{2}$ & $K_{3}$ & $K_{4}$ \\
\hline-1244857.59 & 24766179.46 & -262582.16 & 97799.05 \\
& & & \\
\hline
\end{tabular}

Table 8.19: displays the RMS values of the passive and semi-active rear driver cases. Figure 8.110 is the plot of acceleration of the vehicle body for both passive and semi-active rear driver cases. The tire deflection plot for both cases is shown in Figure 8.111 for the rear driver location. Figure 8.112 is a plot of the suspension deflection for both cases. The EMSA force plot is shown in Figure 8.113. 
Table 8.19: RMS values for the rear driver location

\begin{tabular}{|c|c|c|c|}
\hline & Passive & Semi-Active & $\begin{array}{c}\text { Percent } \\
\text { Improvement }\end{array}$ \\
\hline$\ddot{X}_{s}$ & $3.9274\left(\frac{m}{s^{2}}\right)$ & $1.0871\left(\frac{m}{s^{2}}\right)$ & 72.32 \\
\hline$\ddot{X}_{u}$ & $8.7202\left(\frac{m}{s^{2}}\right)$ & $2.6196\left(\frac{m}{s^{2}}\right)$ & 69.96 \\
\hline$X_{1}$ & $0.007336(m)$ & $0.001459(m)$ & 80.11 \\
\hline$X_{2}$ & $0.002125(m)$ & $0.000517(m)$ & 75.65 \\
\hline$X_{3}$ & $0.1921\left(\frac{m}{s}\right)$ & $0.04582\left(\frac{m}{s}\right)$ & 76.15 \\
\hline$X_{4}$ & $0.08165\left(\frac{m}{s}\right)$ & $0.02919\left(\frac{m}{s}\right)$ & 64.25 \\
\hline
\end{tabular}

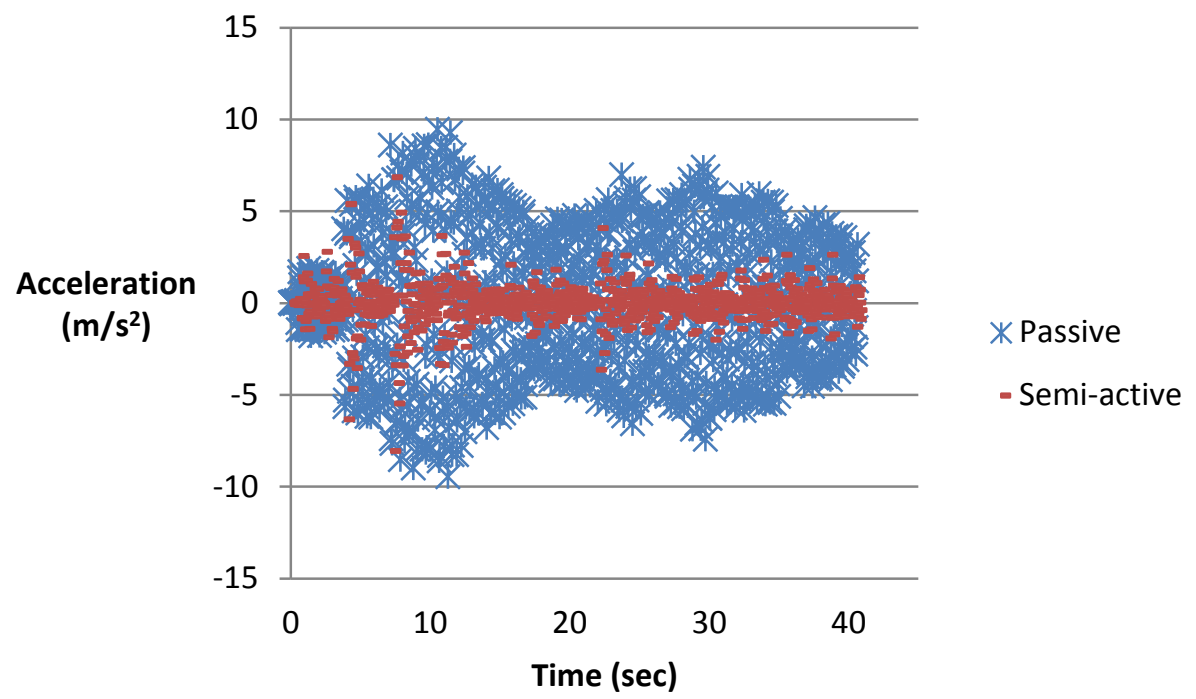

Figure 8.110: Rear driver vehicle body acceleration 


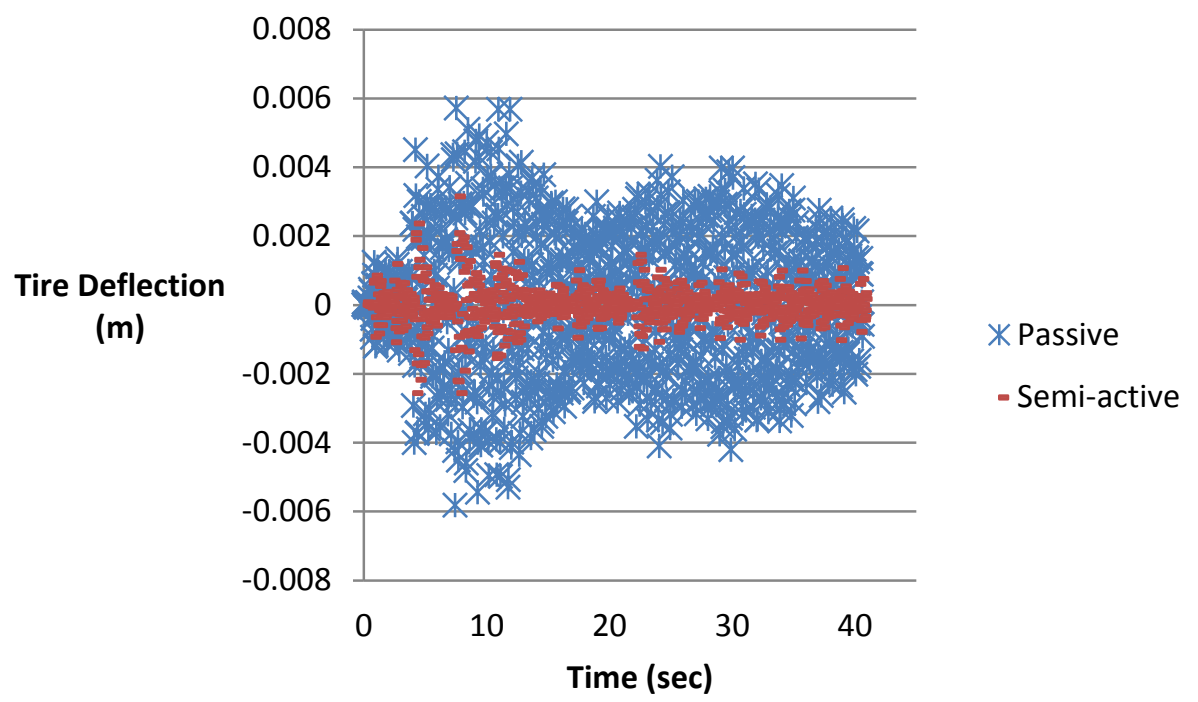

Figure 8.111: Rear driver tire deflection

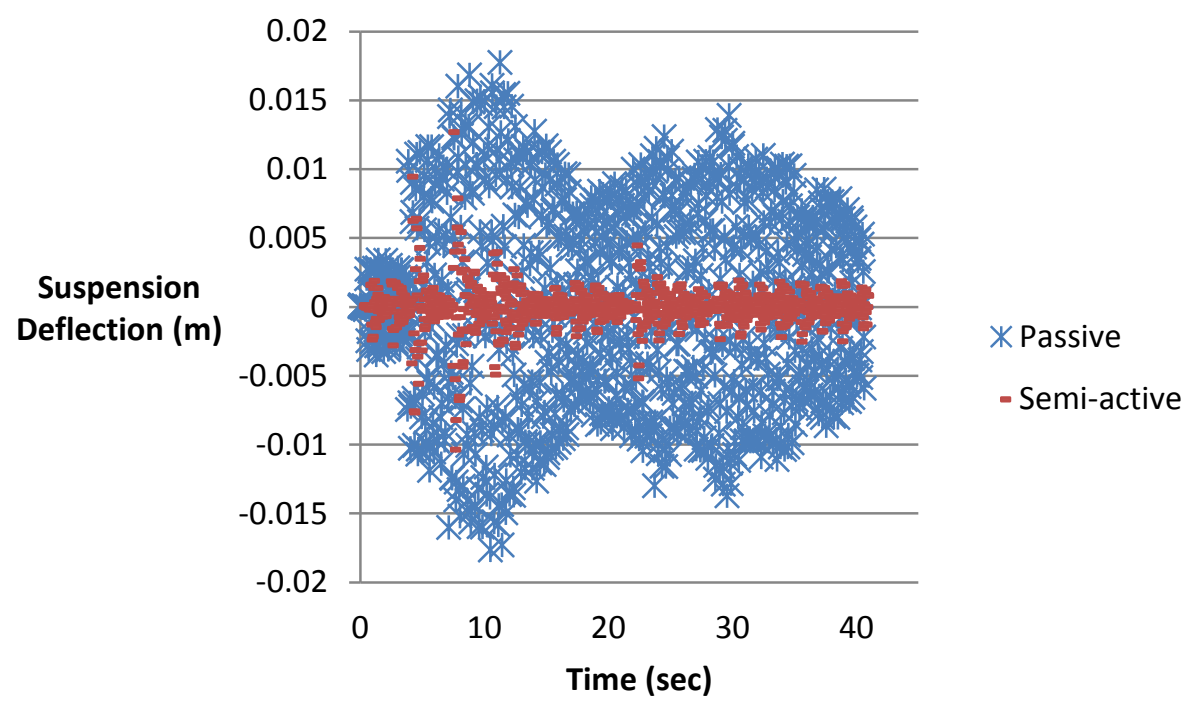

Figure 8.112: Rear driver suspension deflection 


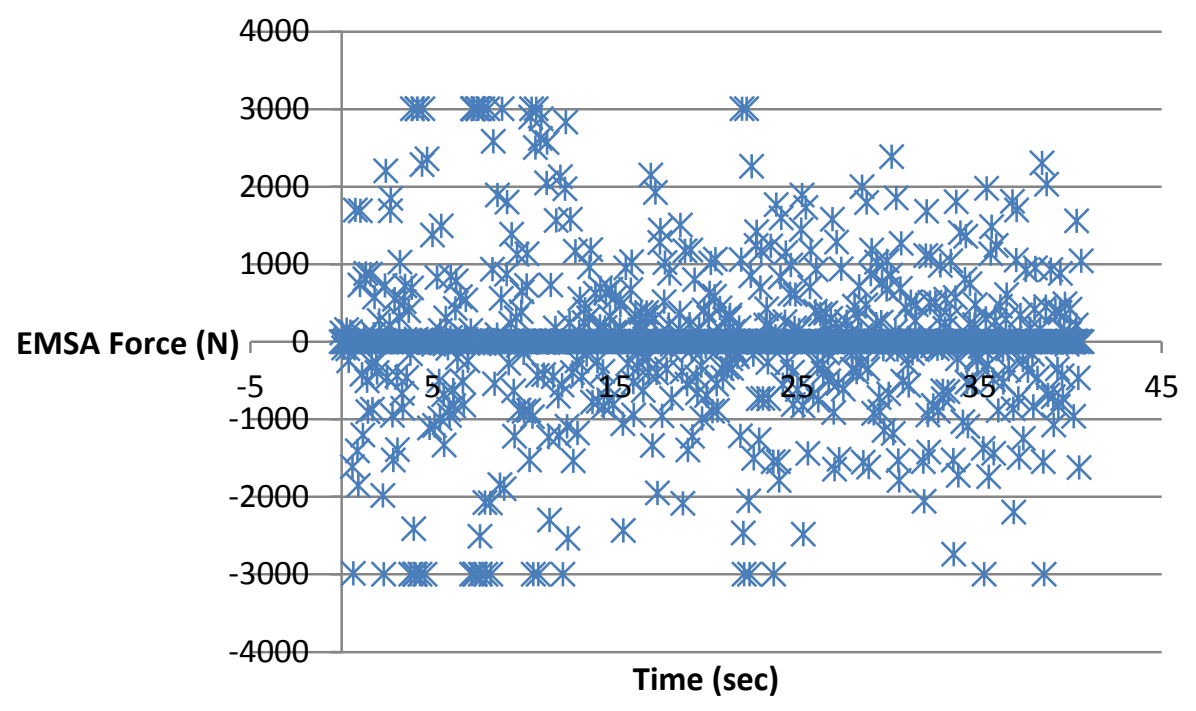

Figure 8.113: EMSA force for rear driver location

Shown in Table 8.20: are the gain values used for the rear passenger semi-active simulation. Notice that these gains are very close in magnitude to the gains shown in Table 8.18:. The plots for the rear passenger location of the acceleration of the vehicle body, $\ddot{x}_{s}$, the tire deflection, $X_{2}$, the EMSA force, and the suspension deflection, $X_{1}$ are omitted for brevity since they nearly match Figures: Figure 8.110, Figure 8.111, Figure 8.112 and Figure 8.113. Table 8.6 displays the RMS values of the passive and semiactive cases.

Table 8.20: Gain values for the rear passenger location

\begin{tabular}{|c|c|c|c|}
\hline$K_{1}$ & $K_{2}$ & $K_{3}$ & $K_{4}$ \\
\hline-1244858 & 24766185 & -262582 & 97799.41 \\
\hline
\end{tabular}

Table 8.21: RMS values for the rear passenger location

\begin{tabular}{|c|c|c|c|}
\hline & Passive & Semi-Active & $\begin{array}{c}\text { Percent } \\
\text { Improvement }\end{array}$ \\
\hline$\ddot{X}_{s}$ & $3.9274\left(\frac{m}{s^{2}}\right)$ & $1.0841\left(\frac{m}{s^{2}}\right)$ & 72.40 \\
\hline$\ddot{X}_{u}$ & $8.7116\left(\frac{m}{s^{2}}\right)$ & $2.6430\left(\frac{m}{s^{2}}\right)$ & 69.66 \\
\hline$X_{1}$ & $0.007336(m)$ & $0.001454(m)$ & 80.17 \\
\hline$X_{2}$ & $0.002125(m)$ & $0.000518(m)$ & 75.63 \\
\hline$X_{3}$ & $0.1921\left(\frac{m}{s}\right)$ & $0.04572\left(\frac{m}{s}\right)$ & 76.20 \\
\hline$X_{4}$ & $0.08159\left(\frac{m}{s}\right)$ & $0.02889\left(\frac{m}{s}\right)$ & 64.59 \\
\hline
\end{tabular}


It is shown from the Matlab-Simulink model that the EMSA does offer an improvement in terms of reducing the acceleration of the sprung and unsprung masses. The front and rear locations have very different mass and suspension properties and that is why their respective gain values are very different. Future work will include the application of high wind loading.

\subsection{References}

1) Gordon, T.J.; "Non-Linear Optimal Control of a Semi-Active Vehicle Suspension System", Chaos, Solutions And Fractals, Vol. 5, No. 9, 1995, pp. 1603-1617.

2) Ley, H. Transportation Research and Analysis Computing Center (TRACC) Year 5 Quarter 2 Progress Report, ANL-11/TRACC-USDOT-Y5Q2, May 2011.

3) Gao, H.; Lam, J.; Wang, C.; "Multi-objective Control of Vehicle Active Suspension Systems Via Load-dependent Controllers," Journal of Sound and Vibration, Vol. 290, 2006, pp. 654-675.

4) Du, H.; Sze, K.Y.; Lam, J.; "Semi-active H[infinity] control of vehicle suspension with magnetorheological dampers", Journal of Sound and Vibration, Vol. 283, No. 3-5, 2005, pp. 981-996.

5) Ley, H. Transportation Research and Analysis Computing Center (TRACC) Year 5 Quarter 3 Progress Report, ANL-11/TRACC-USDOT-Y5Q2, August 2011. 


\section{Issues}

The implementation of the RTSTEP project with the City of Chicago required the temporary assignment of staff working on transportation modeling to this independent project. This assignment does not affect user support, training courses, and other needed support functions. It rather supplements activities that are being conducted at TRACC in this area, and is of high interest to FHWA. The work builds on previous work performed for the Illinois Department of Transportation, and has resulted in a much improved Chicago TRANSIMS model.

TRACC is also evaluating different options for expanding the HPC computing platform. Possible systems may be offered by Cray, Dell, and other vendors. TRACC will work closely with procurement to determine the best mechanism to establish this new capability in a cost-efficient manner. 


\section{Financial Summary - Transportation Research and Analysis Computing Center for USDOT}

\begin{tabular}{|c|c|c|c|c|c|}
\hline & $\begin{array}{l}\text { USDOT } \\
\text { FY2007 } \\
\text { Expenditure }\end{array}$ & $\begin{array}{l}\text { USDOT } \\
\text { FY2008 } \\
\text { Expenditure }\end{array}$ & $\begin{array}{l}\text { USDOT } \\
\text { FY2009 } \\
\text { Expenditure }\end{array}$ & $\begin{array}{l}\text { USDOT } \\
\text { FY2010 } \\
\text { Expenditure }\end{array}$ & $\begin{array}{l}\text { USDOT } \\
\text { FY2011 } \\
\text { Expenditure }\end{array}$ \\
\hline $\begin{array}{l}\text { Computing, Visualization, Networking, and } \\
\text { Outreach Infrastructure, Facilities, and } \\
\text { Capabilities } \\
\text { Effort } \\
\text { Facilities, Hardware, Software and Support } \\
\text { Infrastructure } \\
\text { Subtotal }\end{array}$ & $\begin{array}{l}536 \\
246 \\
782\end{array}$ & $\begin{array}{r}685 \\
1,629 \\
2,314\end{array}$ & $\begin{array}{r}1,269 \\
724 \\
1,993\end{array}$ & $\begin{array}{r}776 \\
566 \\
1,342\end{array}$ & $\begin{array}{r}746 \\
\\
348 \\
1094\end{array}$ \\
\hline $\begin{array}{l}\text { Traffic Modeling \& Simulation and } \\
\text { Emergency Transportation Planning } \\
\text { Effort } \\
\text { Facilities, Hardware, Software and Support } \\
\text { Infrastructure } \\
\text { Subtotal }\end{array}$ & $\begin{array}{r}129 \\
- \\
129\end{array}$ & $\begin{array}{r}- \\
345\end{array}$ & $\begin{array}{r}- \\
553\end{array}$ & $\begin{array}{r}- \\
290\end{array}$ & $\begin{array}{r}- \\
65\end{array}$ \\
\hline $\begin{array}{l}\text { Computational Fluid Dynamics for Hydraulic } \\
\text { and Aerodynamic Research } \\
\text { Effort } \\
\text { Facilities, Hardware, Software and Support } \\
\text { Infrastructure } \\
\text { Subtotal }\end{array}$ & $\begin{array}{r}70 \\
212\end{array}$ & $\begin{array}{l}118 \\
459\end{array}$ & $\begin{array}{l}123 \\
757\end{array}$ & $\begin{array}{l}512 \\
109 \\
621\end{array}$ & $\begin{array}{r}129 \\
18 \\
147\end{array}$ \\
\hline $\begin{array}{l}\text { Multidimensional Data Visualization } \\
\text { Effort } \\
\text { Facilities, Hardware, Software and Support } \\
\text { Infrastructure } \\
\text { Subtotal }\end{array}$ & - & - & 433 & 119 & 41 \\
\hline $\begin{array}{l}\text { Computational Structural Mechanics } \\
\text { Effort } \\
\text { Facilities, Hardware, Software and Support } \\
\text { Infrastructure } \\
\text { Subtotal }\end{array}$ & 60 & $\begin{array}{r}91 \\
236\end{array}$ & $\begin{array}{l}394 \\
294 \\
688\end{array}$ & $\begin{array}{l}472 \\
197 \\
669\end{array}$ & $\begin{array}{l}258 \\
283 \\
541\end{array}$ \\
\hline $\begin{array}{l}\text { Overall Program Totals } \\
\text { Effort } \\
\text { Facilities, Hardware, Software \& Support } \\
\text { Infrastructure } \\
\text { Total }\end{array}$ & $\begin{array}{r}867 \\
\\
316 \\
1,183\end{array}$ & $\begin{array}{l}1,516 \\
1,838 \\
3,354\end{array}$ & $\begin{array}{l}3,283 \\
1,141 \\
4,424\end{array}$ & $\begin{array}{r}2,169 \\
872 \\
3041\end{array}$ & $\begin{array}{r}1239 \\
\\
649 \\
1888\end{array}$ \\
\hline & Authorization & & Cumulative & corization & \\
\hline Y1 (FY06) USDOT & $\$ 2.8$ & & & $\$ 2.8$ & \\
\hline Y2 (FY07) USDOT & $\$ 3.1$ & & & $\$ 5.9$ & \\
\hline Y3 (FY08) USDOT & $\$ 3.1$ & & & $\$ 9.0$ & \\
\hline Y4 (FY09) USDOT & $\$ 4.2$ & & & $\$ 13.2$ & \\
\hline Y5 (FY10) USDOT & $\$ 3.6$ & & & $\$ 16.8$ & \\
\hline
\end{tabular}




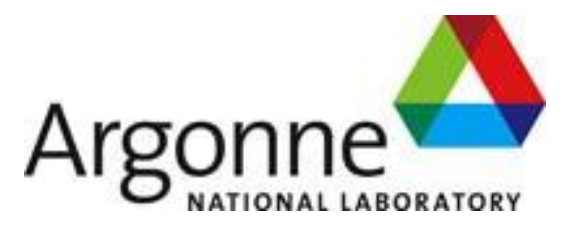

\section{Energy Systems Division}

Argonne National Laboratory

www.anl.gov

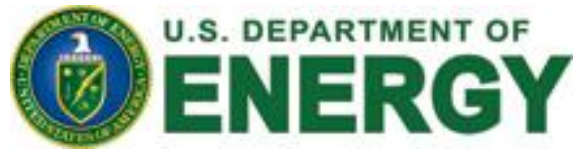

Argonne National Laboratory is a U.S. Department of Energy laboratory managed by UChicago Argonne, LLC 